
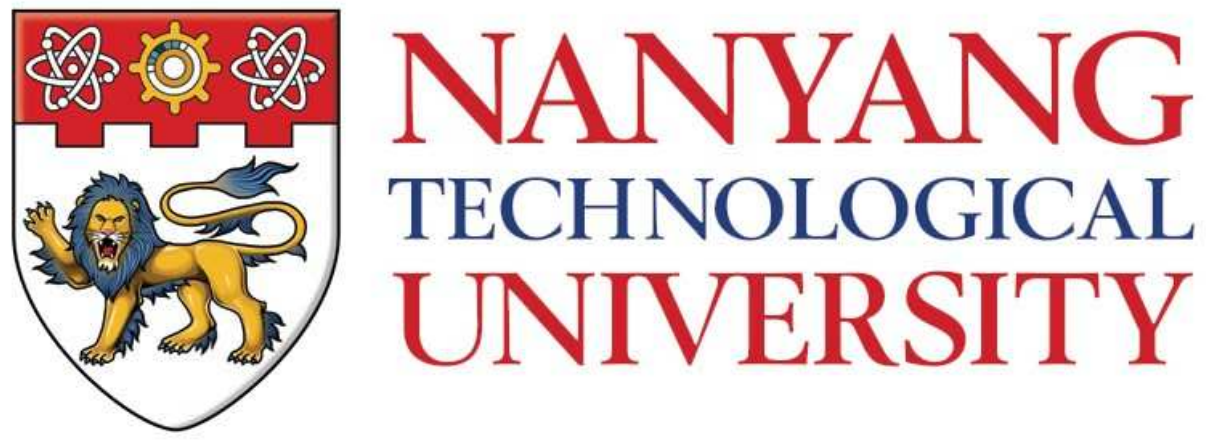

\title{
FLEXIBLE SEMI-PARAMETRIC QUANTILE REGRESSION MODELS
}

\section{FAN ZENGYAN}

DIVISION OF MATHEMATICAL SCIENCES SCHOOL OF PHYSICAL AND MATHEMATICAL SCIENCES 


\title{
FLEXIBLE SEMI-PARAMETRIC QUANTILE REGRESSION MODELS
}

\section{FAN ZENGYAN}

\author{
Division of Mathematical Sciences \\ School of Physical and Mathematical Sciences
}

A thesis submitted to Nanyang Technological University in partial fulfillment of the requirement for the degree of

Doctor of Philosophy in Mathematical Sciences 


\begin{abstract}
This work involves interquantile identification and variable selection in two semi-parametric quantile regression models, an additive model and an additive coefficient model.

In the first part, we investigate the commonality of non-parametric component functions among different quantile levels in additive regression models with fixed dimension. We propose two fused adaptive group LASSO penalties to shrink the difference of functions between neighbouring quantile levels. The proposed methodology is able to simultaneously estimate the non-parametric functions and identify the quantile regions where functions are unvarying, and thus is expected to perform better than standard additive quantile regression when there exists a region of quantile levels on which the functions are unvarying.

In the second part, we consider variable selection in quantile additive coefficient models (ACM) with high dimensionality under a sparsity assumption. First, we consider the oracle estimator for quantile ACM when the number of additive coefficient functions is diverging. Then we adopt the SCAD penalty and investigate the non-convex penalized estimator for model estimation and variable selection. Under some regularity conditions, we prove the oracle estimator is a local solution of the SCAD penalized quantile regression problem.

Simulation studies and real data applications illustrate that the proposed methods in this thesis yield better numerical results than some existing methods.
\end{abstract}




\section{Acknowledgements}

First of all, I would like to express my deep appreciation to my supervisor Prof. Xiang Liming and my co-supervisor Prof. Lian Heng from the City University of Hong Kong. Without their encouragement and guidance, this thesis would be impossible. I am grateful to Prof. Lian Heng, not only for accepting me as a part of his research group even though I did not have any experience in the area of Statistics, but also for offering me a one-year overseas exchange at the University of New South Wales, Sydney. He helped me build up my foundation in research and shared many life lessons with me. Besides my supervisors, I would like to thank my thesis committee member, Prof. Kwoh Chee Keong from the School of Computer Science and Engineering. He generous introduced Bioinformatics to me and his insightful comments helped strengthen this thesis.

My appreciation also goes to the Division of Mathematical Sciences. I am grateful to be able to spend one out of four years in Sydney, Australia and attend an international conference in the US. I thank my seniors, Zhao Kaifeng and Tang Xingyu, for their friendship, help and for all the fun we have had in the last four years.

Last but not least, I would like to thank my parents, who raised me with endless love, and my boyfriend Wee Chin whose faithful support during this Ph.D. journey is so appreciated. Without their love and support, I would never get to this stage. 


\section{List of Works}

Below is the list of work done, in chronological order, during my Ph.D. studies in NTU.

1. Lian, H., Meng, J. and Fan, Z., (2015). "Simultaneous estimation of linear conditional quantiles with penalized splines", Journal of Multivariate Analysis, 141:1-21.

2. Lian, H. and Fan, Z., (2015). "Estimation of a sparse and spiked covariance matrix", Journal of Nonparametric Statistics, 27:241-252.

3. Fan, Z. and Lian, H., (2016). "Minimax convergence rates for kernel CCA", Journal of Multivariate Analysis, 150:183-190.

4. Fan, Z. and Lian, H., (2017). "Interquantile shrinkage in additive models", Journal of Nonparametric Statistics, to appear.

5. Fan, Z. and Lian, H., (2017). "Quantile regression for additive coefficient models in high dimensions", Submitted. 



\section{Contents}

Abstract

Acknowledgements

List of Works $\quad$ V

$\begin{array}{lll}1 & \text { Introduction } & 1\end{array}$

1.1 Quantile Regression . . . . . . . . . . . . . . . . . . . 1

1.2 Two Semi-parametric Quantile Regression Models . . . . . . 4

1.2.1 Additive Quantile Regression Models . . . . . . . . . 5

1.2 .2 Additive Coefficient Quantile Regression Models . . . 6

1.3 Penalty Methods . . . . . . . . . . . . . . . . . . . 8

1.3 .1 Interquantile Shrinkage . . . . . . . . . . . . . . . 10

1.3 .2 Variable Selection . . . . . . . . . . . . . . . 11

1.4 Thesis Outline. . . . . . . . . . . . . . . . . . . . . . . . . . 12

$\begin{array}{|ll|}2 & \text { Interquantile Shrinkage in Additive Models }\end{array}$

2.1 Proposed Methodologies . . . . . . . . . . . . . . . . . 16

$2.1 .1 \quad$ Standard Quantile Regression Estimator . . . . . . . 16 
2.1 .2 Penalized Estimator FAGL1 . . . . . . . . . . . . . . 18

2.1 .3 Penalized Estimator FAGL2 . . . . . . . . . . . . . . 20

2.1 .4 Computation . . . . . . . . . . . . . 20

2.2 Theoretical Results . . . . . . . . . . . . . . . . . . 22

$2.2 .1 \quad$ Standard Quantile Regression Estimator . . . . . . . 22

$2.2 .2 \quad$ Penalized Estimator FAGL1 . . . . . . . . . . . . . . 22

2.2 .3 Penalized Estimator FAGL2 . . . . . . . . . . . . . . 23

2.3 Numerical Results . . . . . . . . . . . . . . . . . . . . . . . . . 24

$2.3 .1 \quad$ Simulation Study . . . . . . . . . . . . . . . 24

2.3 .2 Real Data Analysis . . . . . . . . . . . . . . . . 32

2.4 Summary $\ldots \ldots \ldots \ldots$

2.5 Appendix $\ldots \ldots \ldots \ldots \ldots$

3 Quantile Regression for Additive Coefficient Models in High

Dimensions

3.1 Proposed Methodologies . . . . . . . . . . . . . . . . 50

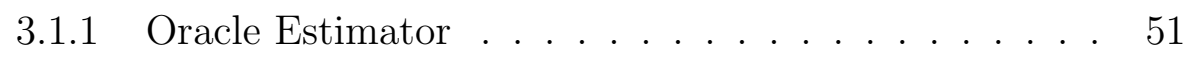

3.1 .2 Group SCAD Penalized Estimator . . . . . . . . . 52

3.1 .3 Computation . . . . . . . . . . . . . 54

3.2 Theoretical Results . . . . . . . . . . . . . . . . . . 55

$3.2 .1 \quad$ Oracle Estimator $\ldots \ldots \ldots$. . . . . . . . . . . 56

3.2 .2 Group SCAD Penalized Estimator $\ldots . . .56$

3.3 Numerical Results . . . . . . . . . . . . . . . . . . . . 57

3.3 .1 Simulation Study . . . . . . . . . . . . . . . . . 57

3.3.2 Genome Wide Association Study . . . . . . . . . . . 59

3.4 Summary $\ldots \ldots \ldots \ldots$

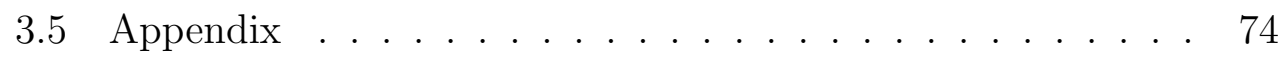




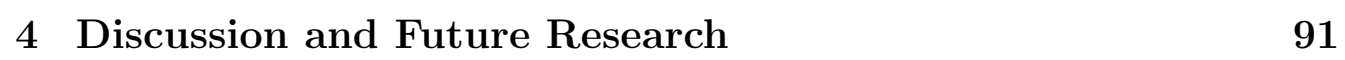

\begin{tabular}{ll}
\hline Bibliography & 95
\end{tabular} 



\section{List of Figures}

$2.1 \quad \mathrm{NO}_{2}$ particles data. Estimated functions with one predictor varying and the others fixed at 0 . The solid black lines are the estimated conditional quantiles by FAGL1 at five different

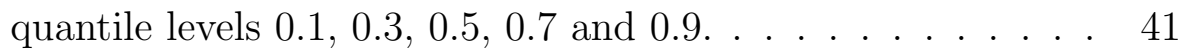

$2.2 \quad \mathrm{NO}_{2}$ particles data. Estimated functions with one predictor varying and the others fixed at 0 . The solid black lines are the estimated conditional quantiles by FAGL2 at five different

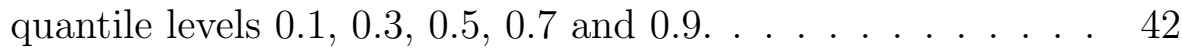

3.1 The SCAD penalized quantile estimates for $\kappa=0$ with standard normal error. Left: Estimates for $\alpha_{41}(\cdot)$ when $\tau=0.5, p_{n}=300$; Right: Estimates for $\alpha_{15,2}(\cdot)$ when $\tau=0.9, p_{n}=100$. The solid curve is the true function and the dotted curves are the estimates for 20 randomly chosen replicates. 
3.2 The SCAD penalized quantile estimates for $\kappa=0$ when the error follows a Student's t distribution with degrees of freedom 3 and scale parameter 1/3. Left: Estimates for $\alpha_{42}(\cdot)$ when $\tau=0.5, p_{n}=600$; Right: Estimates for $\alpha_{15,1}(\cdot)$ when $\tau=0.7, p_{n}=300$. The solid curve is the true function and the dotted curves are the estimates for 20 randomly chosen replicates. . . . . . . . . . . . . . . . . . 65

3.3 The SCAD penalized quantile estimates for $\kappa=1$ when the error follows a standard normal distribution. Left: Estimates for $\alpha_{41}(\cdot)$ when $\tau=0.5, p_{n}=300 ;$ Right: Estimates for $\alpha_{15,2}(\cdot)$ when $\tau=0.7$, $p_{n}=100$. The solid curve is the true function and the dotted curves are the estimates for 20 randomly chosen replicates. . . . . . . . . . 70

3.4 The SCAD penalized quantile estimates for $\kappa=1$ when the error follows a Student's t distribution with degrees of freedom 3 and scale parameter $1 / 3$. Left: Estimates for $\alpha_{42}(\cdot)$ when $\tau=0.5, p_{n}=600$; Right: Estimates for $\alpha_{15,1}(\cdot)$ when $\tau=0.9, p_{n}=100$. The solid curve is the true function and the dotted curves are the estimates for 20 randomly chosen replicates. . . . . . . . . . . . . . . . . . . . 71 


\section{List of Tables}

2.1 Summary of $\sqrt{\widehat{I S E}}$ for non-parametric functions $f_{1}, f_{2}$ and $f_{3}$ in Example $|2.1|$ when $n=100$ and $\epsilon_{i}$ follows a normal distribution with mean 0 and standard deviation 0.5. Standard errors based on simulations are shown in the parentheses. . . 26

2.2 True proportion of correctly identifying the model in Example $2.1 \mid$ when $n=100$ and $\epsilon_{i}$ follows a normal distribution with mean 0 and standard deviation 0.5 . . . . . . . . . 26

$2.3 \quad$ Summary of $P E$ in Example $2.1 \mid$ when $n=100$ and $\epsilon_{i}$ follows a normal distribution with mean 0 and standard deviation 0.5. Standard errors based on simulations are shown in the parentheses. . . . . . . . . . . . . 27

2.4 Summary of $\sqrt{\widehat{I S E}}$ for non-parametric functions $f_{1}, f_{2}$ and $f_{3}$ in Example $|2.1|$ when $n=200$ and $\epsilon_{i}$ follows a normal distribution with mean 0 and standard deviation 0.5 . Standard errors based on simulations are shown in the parentheses. . . 27 
2.5 True proportion of correctly identifying the model in Example $2.1 \mid$ when $n=200$ and $\epsilon_{i}$ follows a normal distribution with mean 0 and standard deviation 0.5 . . . . . . . . . . . 28

2.6 Summary of $P E$ in Example $2.1 \mid$ when $n=200$ and $\epsilon_{i}$ follows a normal distribution with mean 0 and standard deviation 0.5. Standard errors based on simulations are shown in the

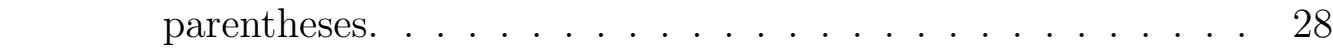

2.7 Summary of $\sqrt{\widehat{I S E}}$ for non-parametric functions $f_{1}, f_{2}$ and \begin{tabular}{|r|l|l|l|}
\hline$f_{3}$ in Example & 2.1 & when $n=100$ and $\epsilon_{i}$ follows a Student's t \\
\hline
\end{tabular} distribution with scale parameter $1 / 3$ and degrees of freedom

3. Standard errors based on simulations are shown in the
parentheses. . . . . . . . . . . . . . . . . . . 29

2.8 True proportion of correctly identifying the model in Example $2.1 \mid$ when $n=100$ and $\epsilon_{i}$ follows a Student's t distribution with scale parameter $1 / 3$ and degrees of freedom $3 . \quad$. . . . 29

2.9 Summary of $P E$ in Example $2.1 \mid$ when $n=100$ and $\epsilon_{i}$ follows a Student's t distribution with scale parameter $1 / 3$ and

degrees of freedom 3. Standard errors based on simulations
are shown in the parentheses. . . . . . . . . . . . . . . 30

2.10 Summary of $\sqrt{\widehat{I S E}}$ for non-parametric functions $f_{1}, f_{2}$ and \begin{tabular}{|r|l|l|l|}
\hline$f_{3}$ in Example & 2.1 & when $n=200$ and $\epsilon_{i}$ follows a Student's t
\end{tabular} distribution with scale parameter $1 / 3$ and degrees of freedom 3. Standard errors based on simulations are shown in the parentheses. . . . . . . . . . . . . . . . . 30

2.11 True proportion of correctly identifying the model in Example $2.1 \mid$ when $n=200$ and $\epsilon_{i}$ follows a Student's t distribution with scale parameter $1 / 3$ and degrees of freedom 3. . . . . . 31 
2.12 Summary of $P E$ in Example 2.1 when $n=200$ and $\epsilon_{i}$ fol-

\begin{tabular}{|c|c|c|}
\hline lows a Student's t distribution with scale parameter $1 / 3$ and \\
\hline
\end{tabular} degrees of freedom 3. Standard errors based on simulations are shown in the parentheses. . . . . . . . . . . . . . 31

2.13 Summary of $\sqrt{\widehat{I S E}}$ for non-parametric functions $f_{1}, f_{2}$ and \begin{tabular}{|r|l|l|l|}
\hline$f_{3}$ in Example & 2.2 & when $n=100$ and $F(\cdot)$ is the normal \\
\hline
\end{tabular} distribution function with mean 0 and standard deviation 0.5. Standard errors based on simulations are shown in the parentheses. . . . . . . . . . . . . . . . . 33

2.14 True proportion of correctly identifying the model in Example $2.2 \mid$ when $n=100$ and $F(\cdot)$ is the normal distribution function with mean 0 and standard deviation 0.5 . . . . . . . 33

\begin{tabular}{|l|l|l|l|}
\hline 2.15 Summary of $P E$ in Example & 2.2 & when $n=100$ and $F(\cdot)$
\end{tabular} is the normal distribution function with mean 0 and standard deviation 0.5. Standard errors based on simulations are shown in the parentheses. . . . . . . . . . . . . . . 34

2.16 Summary of $\sqrt{\widehat{I S E}}$ for non-parametric functions $f_{1}, f_{2}$ and

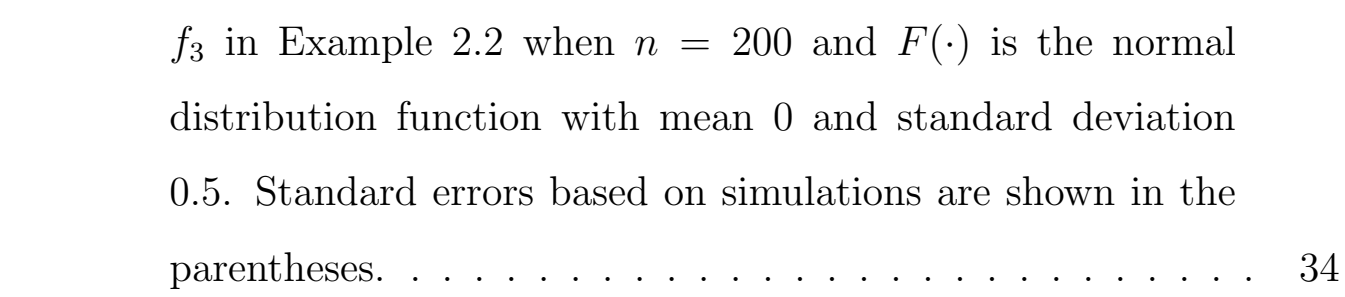

2.17 True proportion of correctly identifying the model in Example 2.2 when $n=200$ and $F(\cdot)$ is the normal distribution function with mean 0 and standard deviation 0.5 . . . . . . 35 
\begin{tabular}{|l|l|l|l|}
\hline 2.18 Summary of $P E$ in Example & 2.2 & when $n=200$ and $F(\cdot)$
\end{tabular} is the normal distribution function with mean 0 and standard deviation 0.5. Standard errors based on simulations are shown in the parentheses. . . . . . . . . . . . . 35

2.19 Summary of $\sqrt{\widehat{I S E}}$ for non-parametric functions $f_{1}, f_{2}$ and \begin{tabular}{|l|l|l|l|}
\hline$f_{3}$ in Example & 2.2 & when $n=100$ and $F(\cdot)$ is the Student's t \\
\hline
\end{tabular} distribution function with scale parameter $1 / 3$ and degrees of freedom 3. Standard errors based on simulations are shown in the parentheses. . . . . . . . . . . . . . 36

2.20 True proportion of correctly identifying the model in Example $2.2 \mid$ when $n=100$ and $F(\cdot)$ is the Student's t distribution function with scale parameter $1 / 3$ and degrees of freedom 3.

\begin{tabular}{|l|l|l|l|}
\hline 2.21 Summary of $P E$ in Example & 2.2 & when $n=100$ and $F(\cdot)$ \\
\hline
\end{tabular} is the Student's t distribution function with scale parameter \begin{tabular}{|ll|}
\hline $1 / 3$ and degrees of freedom 3. Standard errors based on \\
\hline
\end{tabular} simulations are shown in the parentheses. . . . . . . . . . 37

2.22 Summary of $\sqrt{\widehat{I S E}}$ for non-parametric functions $f_{1}, f_{2}$ and \begin{tabular}{|r|l|l|l|}
\hline$f_{3}$ in Example & 2.2 & when $n=200$ and $F(\cdot)$ is the Student's t \\
\hline
\end{tabular} distribution function with scale parameter $1 / 3$ and degrees of freedom 3. Standard errors based on simulations are shown [in the parentheses. . . . . . . . . . . . . . . . . 37

2.23 True proportion of correctly identifying the model in Exam\begin{tabular}{|r|r|l|l|}
\hline ple & 2.2 & when $n=200$ and $F(\cdot)$ is the Student's t distribution \\
\hline
\end{tabular} \begin{tabular}{|c|c|}
\hline function with scale parameter $1 / 3$ and degrees of freedom 3. & 38
\end{tabular} 
\begin{tabular}{|l|l|l|l|}
\hline 2.24 Summary of $P E$ in Example & 2.2 & when $n=200$ and $F(\cdot)$
\end{tabular} is the Student's t distribution function with scale parameter $1 / 3$ and degrees of freedom 3. Standard errors based on simulations are shown in the parentheses. . . . . . . . . . . 38

$2.25 \mathrm{NO}_{2}$ particles data. The proportion of times the interquantile differences are shrunk to zero. . . . . . . . . . . . . . . . 40

3.1 Simultaion results for $\kappa=0$ when $\epsilon_{i}$ follows a standard normal distribution and $\tau=0.5$. Standard deviations computed based on 200 repetitions are shown in the parentheses. . . . 60

3.2 Simulation results for $\kappa=0$ when $\epsilon_{i}$ follows a standard nor-

mal distribution and $\tau=0.7,0.9$. Standard deviations are
shown in the parentheses. . . . . . . . . . . . . . . 61

$3.3 \quad$ Simultaion results for $\kappa=0$ when $\tau=0.5$ and the error $\epsilon_{i}$ follows a Student's t distribution with scale parameter $1 / 3$ and degrees of freedom 3. Standard deviations are shown in the parentheses. . . . . . . . . . . . . . . 62

$3.4 \quad$ Simulation results for $\kappa=0$ when $\tau=0.7,0.9$ and the error \begin{tabular}{|c|}
\hline$\epsilon_{i}$ follows a Student's t distribution with scale parameter 1/3 \\
\hline and degrees of freedom 3. Standard deviations are shown in \\
\hline
\end{tabular} the parentheses. . . . . . . . . . . . . . . 63

3.5 Simultaion results for $\kappa=1$ when $\epsilon_{i}$ follows a standard normal distribution and $\tau=0.5$. Standard deviations are shown in the parentheses. . . . . . . . . . . . . . . 66

3.6 Simulation results for $\kappa=1$ when $\epsilon_{i}$ follows a standard normal distribution and $\tau=0.7,0.9$. Standard deviations are shown in the parentheses. . . . . . . . . . . . . . . 67 
3.7 Simultaion results for $\kappa=1$ when $\tau=0.5$ and the error $\epsilon_{i}$ follows a Student's t distribution with scale parameter $1 / 3$ and degrees of freedom 3. Standard deviations are shown in the parentheses. . . . . . . . . . . . . . . . . . . . . . . 68

3.8 Simulation results for $\kappa=1$ when $\tau=0.7,0.9$ and the error $\epsilon_{i}$ follows a Student's t distribution with scale parameter $1 / 3$ and degrees of freedom 3. Standard deviations are shown in the parentheses. . . . . . . . . . . . . . . . . . . . . . 69

3.9 Quantile regresstion analysis of weaning weight base on the sheep data. . . . . . . . . . . . . . . . . . . . 73 
Chapter 1

\section{Introduction}

In recent years, the idea of quantile regression has become very popular in statistical literature, from frequentist approaches to Bayesian approaches. In the literature, this appealing method has mostly been considered in linear regression models. However, the assumption made on linear models is sometimes strict and unrealistic, especially the dataset from real world applications. In this thesis, we focus on quantile regression for two semiparametric models, which are more flexible than linear models.

In this chapter, we first introduce quantile regression in Section 1.1, followed by two semi-parametric quantile regression models in Section 1.2. A review on penalty methods is given in Section 1.3 . This chapter is concluded with a thesis outline in Section 1.4 .

\subsection{Quantile Regression}

Let us consider a random sample $\left(Y_{i}, \mathbf{X}_{i}\right), i=1, \ldots, n$, which are independent and identically distributed as $(Y, \mathbf{X})$. Regression analysis is concerned with the question of how the univariate response $Y$ can be explained by 
the $p$-dimensional predictor variables $\mathbf{X}$. Linear regression models are the commonly used models, which consider the regression function $\mathrm{E}(Y \mid \mathbf{X})$ in a linear way,

$$
\mathrm{E}(Y \mid \mathbf{X})=\alpha+\mathbf{X} \boldsymbol{\beta}
$$

where $\mathrm{E}(Y \mid \mathbf{X})$ is the mean function of $Y$ given $\mathbf{X}, \alpha$ is the intercept term, and $\boldsymbol{\beta} \in \mathbb{R}^{p}$. Denote by $\epsilon=Y-\mathrm{E}(Y \mid \mathbf{X})$ the deviation of $Y$ from the conditional mean function $\mathrm{E}(Y \mid \mathbf{X})$. Eventually, the linear regression models are given by

$$
Y=\alpha+\mathbf{X} \boldsymbol{\beta}+\epsilon
$$

Regarding the linear regression problem (1.2), how to estimate the parameters $\alpha, \boldsymbol{\beta}$ and illustrate the regression function are fundamental issues in regression analysis. A widely used approach is the ordinary least squares regression, which considers minimizing the sum of squared residuals,

$$
\min _{\alpha, \boldsymbol{\beta}} \sum_{i=1}^{n}\left(Y_{i}-\alpha-\mathbf{X}_{i} \boldsymbol{\beta}\right)^{2}
$$

The estimates are obtained by considering a quadratic loss, thus they are highly influenced by outliers. When the error term $\epsilon_{i}$ is heavy-tailed, the mean estimates will be unstable as well. Alternatively, the least absolute deviations regression is proposed to consider minimizing the sum of absolute value of residuals, that is,

$$
\min _{\alpha, \boldsymbol{\beta}} \sum_{i=1}^{n}\left|Y_{i}-\alpha-\mathbf{X}_{i} \boldsymbol{\beta}\right|,
$$

and the estimates are also known as median estimates. When we investigate low birth weight or low household income problem, we want to extract more information than the ordinary least squares regression or the least absolute deviations regression. For example, infants born with low weight are more 
likely to have health problems in their later years. Thus, researchers usually explore 5 or 10 quantiles of birth weight to invesitgate the low birth weight problem. Koenker and Bassett (1978) proposed quantile regression, which has become a useful method in statistical theory and application. Quantile regression is interested in the regression function, the $\tau$-th conditional quantile $Q_{\tau}(Y \mid \mathbf{X})$, which is given by

$$
Q_{\tau}(Y \mid \mathbf{X})=\inf \{y: P(Y \leq y \mid \mathbf{X}) \geq \tau\}
$$

Therefore, the linear quantile regression model can be formulated as

$$
Q_{\tau}(Y \mid \mathbf{X})=\alpha+\mathbf{X} \boldsymbol{\beta}
$$

In the procedure of quantile regression for 1.3, the estimates $\hat{\alpha}_{\tau}$ and $\hat{\boldsymbol{\beta}}_{\tau}$ are obtained by minimizing the sum of check loss at a given quantile level $\tau$,

$$
\sum_{i=1}^{n} \rho_{\tau}\left(Y_{i}-\alpha-\mathbf{X}_{i} \boldsymbol{\beta}\right),
$$

where $\rho_{\tau}(u)=u(\tau-I(u<0)), u$ is the residual and $I(\cdot)$ is the indicator function. It is more robust than the ordinary least squares regression when the distribution of random errors $\epsilon_{i}$ is heavy-tailed, where $\epsilon:=Y-Q_{\tau}(Y \mid \mathbf{X})$ for quantile regression. Among various quantile levels, quantile regression estimates also provide a more comprehensive view of the entire conditional distribution of the response when the data is heteroscedastic. Therefore, quantile regression has been applying to diverse areas, including public health (Abrevaya (2001)) and financial economics (Buchinsky (1994)).

In the literature, quantile regression for linear models has been well studied (Bondell et al. (2010): Belloni and Chernozhukov (2011): Wang et al. (2012)). However, the work on semi-parametric models has been limited. 
Kim (2007); Wang et al. (2009); Zhao and Lian (2017) extended quantile regression to various semi-parametric models with fixed dimension. In this thesis, we focus on two semi-parametric quantile regression models that we will introduce in the following Sections.

\subsection{Two Semi-parametric Quantile Regres- sion Models}

The linear regression (1.2) has a simple interpretation, thus it has been widely used in statistical learning. Sometimes, the predetermined linear structure cannot illustrate the real data well in applications. Therefore, a trend in the past few decades has been to move away from the linear way. Non-parametric regression models are proposed to eliminate the assumption on the linear dependency and consider the regression function $\mathrm{E}(Y \mid \mathbf{X})$ in a non-parametric way,

$$
\mathrm{E}(Y \mid \mathbf{X})=m(\mathbf{X})
$$

where $m(\cdot)$ is a multivariate smooth function. The non-parametric regression model (1.4) is much more flexible than the linear regression model 1.1 in practice. However, the optimal rate of convergence for $m(\cdot)$ could be very slow when the dimension of predictor variables $\mathbf{X}$ increases. In this situation, we often encounter the difficulty known as "curse of dimensionality". In the literature, lots of efforts have been working on simplifing the model structure to make statistical inference possible (Xia et al. (2002)), such as function approximation and dimension reduction. Several semi-parametric models have been proposed to alleviate the curse of dimensionality. In this thesis, we will introduce additive models and additive coefficient models for 
quantile regression.

\subsubsection{Additive Quantile Regression Models}

Additive models, which were first suggested by Friedman and Stuetzle (1981), are commonly used in multivariate statistical learning to overcome the "curse of dimensionality". The additive models are proposed to consider the regression function $\mathrm{E}(Y \mid \mathbf{X})$ with the form which is a sum of univariate smooth functions, that is,

$$
\mathrm{E}(Y \mid \mathbf{X})=\alpha+\sum_{j=1}^{p} f_{j}\left(X_{j}\right),
$$

where $f_{j}(\cdot)$ 's are univariate smooth functions. Thus, the additive models are presented by

$$
Y=\alpha+\sum_{j=1}^{p} f_{j}\left(X_{j}\right)+\epsilon,
$$

where $\epsilon:=Y-\mathrm{E}(Y \mid \mathbf{X})$. The model (1.6) is a special case of the projection pursuit regression (Friedman and Stuetzle (1981)), and it becomes the linear model (1.2) when $f_{j}(\cdot)$ 's are linear functions. With the additive structure, they are much more practical than linear regression models (Breiman and Friedman (1985); Stone (1985); Buja et al. (1989)).

Stone $(1985)$ proved that the additive components $f_{j}(\cdot)$ can be estimated at the optimal rate of convergence. The backfitting algorithm (Friedman and Stuetzle (1981); Buja et al. (1989)) was proposed to approximate the additive components $f_{j}(\cdot)$, but this iterative procedure is hard to develop asymptotic theory. A variety of methods have been introduced for the estimation of additive regression models, including Linton and Nielsen (1995); Xue (2009); Wang et al. (2011). However, these works are limited by their focus on the conditional mean function (1.5). 
In this thesis, we will study additive quantile regression models, which consider the $\tau$-th conditional quantile $Q_{\tau}(\mathbf{X})$ instead of the conditional mean function (1.5),

$$
Q_{\tau}(\mathbf{X})=\mu_{\tau}+\sum_{j=1}^{p} f_{j}^{\tau}\left(X_{j}\right)
$$

where $\mu_{\tau}$ and $f_{j}^{\tau}(\cdot)$ depend on quantile level $\tau$. Koenker et al. (1994) employed a total variation smoothing penalty for univariate components and Koenker and Mizera (2004) further explored bivariate function estimation. The additive quantile regression models $(1.7)$ were also considered in De Gooijer and Zerom (2003) and Horowitz and Lee (2005), and a Bayesian approach was laid out in Waldmann et al. (2013).

\subsubsection{Additive Coefficient Quantile Regression Mod- els}

Additive coefficient models (ACM), with linear dependence on some predictor variables and nonlinear non-parametric dependence on others, were proposed by Xue and Yang (2006b). More specifically, let $\left(Y_{i}, \mathbf{X}_{i}, \mathbf{T}_{i}\right)$, $i=1, \ldots, n$, be random variables that are independent and identically distributed as $(Y, \mathbf{X}, \mathbf{T})$, where $Y$ is the univariate response variable and $\mathbf{X}=\left(X_{1}, \ldots, X_{d}\right)^{\mathrm{T}}$ and $\mathbf{T}=\left(T_{1}, \ldots, T_{p}\right)^{\mathrm{T}}$ are predictor variables. The additive coefficient models are proposed to consider the regression function $\mathrm{E}(Y \mid \mathbf{X}, \mathbf{T})$,

$$
\mathrm{E}(Y \mid \mathbf{X}, \mathbf{T})=\sum_{l=1}^{p} \alpha_{l}(\mathbf{X}) T_{l}, \quad \alpha_{l}(\mathbf{X})=\alpha_{l 0}+\sum_{s=1}^{d} \alpha_{l s}\left(\mathbf{X}_{s}\right)
$$


where $\alpha_{l s}(\cdot)$ are univariate smooth functions. Eventually, the additive coefficient models are given by

$$
Y=\sum_{l=1}^{p}\left\{\alpha_{l 0}+\sum_{s=1}^{d} \alpha_{l s}\left(\mathbf{X}_{s}\right)\right\} T_{l}+\epsilon
$$

where $\epsilon:=Y-\mathrm{E}(Y \mid \mathbf{X}, \mathbf{T})$. With the additive structure on coefficients $\alpha_{l}(\cdot)$, this semiparametric model $(1.9)$ enables a simple interpretation and alleviates the "curse of dimensionality" in high-dimensional cases. The model (1.9) includes a special case of the varying coefficient models (Hastie and Tibshirani (1993)) and additive models (1.6). Additive coefficient models can be used for analyzing economic time series data and gene-environment interaction, for example, to obtain better fitting model than linear regression models.

A variety of methods have been introduced for the estimation of additive coefficient models. Xue and Yang (2006a) applied polynomial splines to fit the model with fixed dimension, and Liu and Yang (2010) extended the spline-backfitted kernel smoothing (Wang and Yang (2007)) to ACM. These works are nevertheless limited to the conditional mean function (1.8. Alternatively, we focus on additive coefficient quantile regression models, which consider the $\tau$-th conditional quantile $Q_{\tau}(\mathbf{X}, \mathbf{T})$,

$$
Q_{\tau}(\mathbf{X}, \mathbf{T})=\sum_{l=1}^{p} \alpha_{l}^{\tau}(\mathbf{X}) T_{l}, \quad \alpha_{l}^{\tau}(\mathbf{X})=\alpha_{l 0}^{\tau}+\sum_{s=1}^{d} \alpha_{l s}^{\tau}\left(\mathbf{X}_{s}\right)
$$

where $\alpha_{l 0}^{\tau}$ and $\alpha_{l s}^{\tau}(\cdot)$ depend on quantile level $\tau$. Motivated by the study of gene and environment interactions, we consider model 1.10 with high dimensions. 


\subsection{Penalty Methods}

This section gives an introduction to the penalty methods. This line of works started from Tibshirani (1996) who proposed Least Absolute Shrinkage and Selection Operator (LASSO) for the linear model (1.2). The LASSO penalized estimators are given by

$$
\hat{\alpha}, \hat{\boldsymbol{\beta}}_{\mathrm{LASSO}}=\underset{\alpha, \boldsymbol{\beta}}{\arg \min } \sum_{i=1}^{n}\left(Y_{i}-\alpha-\mathbf{X}_{i} \boldsymbol{\beta}\right)^{2}+\lambda_{n} \sum_{j=1}^{p}\left|\beta_{j}\right|,
$$

where $\lambda_{n} \geq 0$ is the tuning parameter controlling the degree of penalization. With this $l_{1}$ constraint in (1.11), it turns to produce some $\beta_{j}$ that are shrunk to zero exactly. Zhao and Yu (2006) showed that this procedure based on $l_{1}$ penalization in general gives inconsistent variable selection results. Fan and Li (2001) proposed Smoothly Clipped Absolute Deviation (SCAD) penalty, which enjoys the oracle properties (unbiasedness, sparsity and continuity). The SCAD penalized estimators for the linear model $(1.2)$ are presented by,

$$
\hat{\alpha}, \hat{\boldsymbol{\beta}}_{\mathrm{SCAD}}=\underset{\alpha, \boldsymbol{\beta}}{\arg \min } \sum_{i=1}^{n}\left(Y_{i}-\alpha-\mathbf{X}_{i} \boldsymbol{\beta}\right)^{2}+\sum_{j=1}^{p} p_{\lambda_{n}}\left(\left|\beta_{j}\right|\right),
$$

where $p_{\lambda_{n}}^{\prime}(t)=\lambda_{n}\left\{I\left(t \leq \lambda_{n}\right)+\frac{\left(a \lambda_{n}-t\right)_{+}}{(a-1) \lambda_{n}} I\left(t>\lambda_{n}\right)\right\}$, for some $a>2$ and $\lambda_{n}>$ 0 . The estimator $\hat{\boldsymbol{\beta}}_{\mathrm{SCAD}}$ can consistently identify the nonzero coefficients and the asymptotic distribution of the nonzero coefficient as the same as the least squares estimator using only predictors associated with nonzero coefficients. Zou (2006) proposed adaptive LASSO, which also enjoys the oracle property as the SCAD penalty does. The adaptive LASSO estimates are given by

$$
\hat{\alpha}, \hat{\boldsymbol{\beta}}_{\mathrm{ALASSO}}=\underset{\alpha, \boldsymbol{\beta}}{\arg \min } \sum_{i=1}^{n}\left(Y_{i}-\alpha-\mathbf{X}_{i} \boldsymbol{\beta}\right)^{2}+\lambda_{n} \sum_{j=1}^{p} \omega_{j}\left|\beta_{j}\right|,
$$


where the weight $\omega_{j}=\left|\tilde{\beta}_{j}\right|^{-\gamma}, \gamma>0$, and $\tilde{\beta}_{j}$ is a $\sqrt{n}$-consistent estimator to $\beta_{j}$. Some other variants of LASSO exist which are related to the penalty used in the current work. Tibshirani et al. (2005) introduced the fused LASSO, which assumes a natural order of predictors exists and penalizes the difference of neighboring coefficients. The fused LASSO estimates $\hat{\alpha}, \hat{\boldsymbol{\beta}}_{\text {FLASSO }}$ are presented by

$\hat{\alpha}, \hat{\boldsymbol{\beta}}_{\mathrm{FLASSO}}=\underset{\alpha, \boldsymbol{\beta}}{\arg \min } \sum_{i=1}^{n}\left(Y_{i}-\alpha-\mathbf{X}_{i} \boldsymbol{\beta}\right)^{2}+\lambda_{n 1} \sum_{j=1}^{p}\left|\beta_{j}\right|+\lambda_{n 2} \sum_{j=2}^{p}\left|\beta_{j}-\beta_{j-1}\right|$,

where $\lambda_{n 1}, \lambda_{n 1} \geq 0$ are tuning parameters.

In some circumstances, we are interested in the regression problem in group manner, such as multifactor analysis of variance. Then, the conditional mean function (1.1) can be formulated as

$$
\mathrm{E}(Y \mid \mathbf{X})=\alpha+\sum_{j=1}^{J} \mathbf{X}_{j} \boldsymbol{\beta}_{j}
$$

where $\mathbf{X}_{j}$ is a $n \times d_{j}$ matrix corresponding to the $j$-th group, $\boldsymbol{\beta}_{j} \in \mathbb{R}^{d_{j}}$ and $j=1, \ldots, J$. Yuan and Lin (2006) proposed group LASSO when the predictors are grouped, and the estimators are defined as the solution to

$$
\hat{\alpha}, \hat{\boldsymbol{\beta}}_{\mathrm{GLASSO}}=\underset{\alpha, \boldsymbol{\beta}}{\arg \min } \sum_{i=1}^{n}\left(Y_{i}-\alpha-\sum_{j=1}^{J} \mathbf{X}_{i j} \boldsymbol{\beta}_{j}\right)^{2}+\lambda_{n} \sum_{j=1}^{J}\left\|\boldsymbol{\beta}_{j}\right\|_{\mathbf{K}_{j}}
$$

where $\left\|\boldsymbol{\beta}_{j}\right\|_{\mathbf{K}_{j}}^{2}=\boldsymbol{\beta}_{j}^{\mathrm{T}} \mathbf{K}_{j} \boldsymbol{\beta}_{j}$ given the positive definite matrix $\mathbf{K}_{j} \in \mathbb{R}^{d_{j} \times d_{j}}$, and $\lambda_{n} \geq 0$ is the tuning parameter for selecting group predictors. When matrix $\mathbf{K}_{j}$ is chosen to be the identity matrix, $\left\|\boldsymbol{\beta}_{j}\right\|_{\mathbf{K}_{j}}$ becomes the Euclidean norm of $\boldsymbol{\beta}_{j}$.

Then, researchers move on to the challenging high dimensional regression problems. Zhang (2010) proposed minimax concave penalty (MCP) 
and considered variable selection in high dimensional linear regression models 1.2 with $p \gg n$. The estimator $\hat{\boldsymbol{\beta}}_{\mathrm{MCP}}$ is able to select the predictors consistently, and it is given by

$$
\hat{\alpha}, \hat{\boldsymbol{\beta}}_{\mathrm{MCP}}=\underset{\alpha, \boldsymbol{\beta}}{\arg \min } \sum_{i=1}^{n}\left(Y_{i}-\alpha-\mathbf{X}_{i} \boldsymbol{\beta}\right)^{2}+\sum_{j=1}^{p} p_{\lambda_{n}}\left(\left|\beta_{j}\right|\right),
$$

where $p_{\lambda_{n}}\left(\left|\beta_{j}\right|\right)=\lambda_{n}\left(\left|\beta_{j}\right|-\frac{\beta_{j}^{2}}{2 a \lambda_{n}}\right) I\left(0 \leq\left|\beta_{j}\right|<a \lambda_{n}\right)+\frac{a \lambda_{n}^{2}}{2} I\left(\left|\beta_{j}\right| \geq a \lambda_{n}\right)$, for some $a>1$ and $\lambda_{n}>0$. However, the penalization methods are not limited to the above-mentioned penalties.

A number of recent papers that have been published focus on extending penalty related procedures to semi-parametric regression models. Huang et al. (2010) applied adaptive group LASSO to the variable selection problem in additive models. Zhang et al. (2011); Lian et al. (2012); Lian (2012) used group LASSO penalty or SCAD penalty to identify the linear components in additive models. In this thesis, we extend penalty methods to conduct interquantile shrinkage and variable selection in two semi-parametric quantile regression models correspondingly.

\subsubsection{Interquantile Shrinkage}

Standard additive quantile regression estimates the non-parametric components in 1.7) for different quantile levels separately. In some situations, it is reasonable to expect that the non-parametric functions may vary across some quantile levels but remain unvarying within other levels. Or, some covariates may have varying effects for different quantile levels and others may have the same effects across all quantile levels.

It would be desirable to develop an interquantile shrinkage method that can take advantage of the property that the non-parametric functions are 
the same at different quantile levels and thus we can combine information across multiple quantile levels to achieve better estimation accuracy. Indeed, for linear quantile regression models (1.3), Jiang et al. (2013) developed two types of fusion penalties to identify the differences of coefficients between neighboring quantile levels. Jiang et al. (2014) further added another penalty to consider variable selection. It is remarkable that these shrinkage methods lead to more efficient estimation in linear quantile regression models, as demonstrated in those papers. To our knowledge, there is currently no study on the identification of the commonality of nonparametric components in additive quantile regression models.

One of our missions is to develop an interquantile shrinkage method that can estimate the non-parametric functions and shrink the component functions at adjacent quantile levels towards each other, in additive quantile regression models (1.7).

\subsubsection{Variable Selection}

Variable selection is a significant part in regression problems. Misspecification, including left out predictors and irrelevant predictors, will cause biasness and lead to poor prediction. As dimension increases, it is desirable to remove predictors that are not related to responses. Stepwise selection and best subset selection are the commonly used techniques for variable selection. Although they are easy to implement, these techniques often suffer some drawbacks, such as computational infeasibility and instability, when the dimension of covariates $p$ increases. Alternatively, the above-mentioned penalty methods can assist us to conduct model estimation and variable selection simultaneously. For the penalized regression problem (1.11), the variable selection procedure obtained by the LASSO 
means that the estimator set $\hat{\mathcal{A}}=\mathcal{A}_{0}$ with large probability if we know the true set $\mathcal{A}_{0}=\left\{j:\left|\beta_{j}\right| \neq 0, j=1, \ldots, p\right\}$ beforehand. As we mentioned, the procedure obtained by the LASSO needs the Irrepresentable Condition to guarantee the selection consistency, but this condition is hard to be satisfied in real applications.

Several papers have been working on extending the above-mentioned penalties to high-dimensional regression models. Belloni and Chernozhukov (2011) applied $l_{1}$ penalty for model selection and estimation in high-dimensional sparse linear models, where the number of predictors is possibly larger than the sample size, but the number of predictors related to the response grows more slowly than the sample size. Wang et al. (2012) investigated SCAD and MCP penalized linear quantile regression in high dimension. Fan et al. (2014a) considered an adaptive LASSO penalized linear quantile regression in high dimension. Sherwood and Wang (2016) extended penalized quantile regression to the partially linear additive models.

Another mission of this thesis is that we consider variable selection for additive coefficient quantile regression models (1.10) in high dimensions. With high dimensionality, we allow the number of predictors to be larger than the sample size $n$ and the number of predictors related to the response grow slowly with the sample size $n$.

\subsection{Thesis Outline}

The rest of this thesis is organised into four chapters as follows.

In Chapter 2, we use fused adaptive group LASSO penalty with B-spline expansion to estimate non-parametric functions and identify quantile regions where non-parametric components are varying or unvarying. We pro- 
pose two fused adaptive group LASSO penalties, one that penalizes each interquantile difference of each non-parametric component (FAGL1) and the other that penalizes all interquantile differences of each non-parametric component as one group (FAGL2). We show that the penalized quantile regression estimators achieve the optimal rate of convergence and consistently identify the stretch of quantile levels on which the predictors have unvarying effects with probability approaching 1 .

In Chapter 3, we apply the group SCAD penalty for variable selection in quantile additive coefficient models. This non-convex penalty, combined with non-smooth quantile loss function and high dimensionality, makes our work challenging. We use B-splines, which is more convenient to implement than the kernel method in high dimensions, to approximate the non-parametric coefficient functions. Under some regularity conditions, we prove the oracle estimator is a local solution of the SCAD penalized quantile regression problem. Simulation studies and genome wide association study show that the proposed method yields a better fitted model than the linear method.

In Chapter 4, we conclude this thesis with discussions, as well as some directions of future work.

All the theoretical proofs in this thesis are attached at the end of each Chapter, and the numerical studies are implemented in $\mathrm{R}$ and available upon request. 

Chapter 2

\section{Interquantile Shrinkage in Additive} Models

Let $\left(Y_{i}, \mathbf{X}_{i}\right), i=1, \ldots, n$, be observed pairs that are independent and identically distributed (i.i.d), where $Y_{i}$ is the response variable and $\mathbf{X}_{i}=$ $\left(X_{i 1}, \ldots, X_{i p}\right)^{T}$ is a $p$-dimensional covariate vector. We are interested in regression at $K$ different quantile levels $0<\tau_{1}<\cdots<\tau_{K}<1$, where $K$ is

a fixed integer. Typically, we use the equally spaced quantiles $\tau_{k}:=\frac{k}{K+1}$, for $k=1, \ldots, K$. Denote by $Q_{\tau_{k}}(\mathbf{X})$ the $\tau_{k}$-th conditional quantile function of $Y$ given $\mathbf{X}$, that is,

$$
Q_{\tau_{k}}(\mathbf{X})=\inf \left\{y: \mathrm{P}(Y \leq y \mid \mathbf{X}) \geq \tau_{k}\right\}
$$

for $k=1, \ldots, K$.

In this chapter, we consider the additive quantile regression model

$$
Q_{\tau_{k}}\left(\mathbf{X}_{i}\right)=\mu_{k}+\sum_{j=1}^{p} f_{j}^{k}\left(X_{i j}\right)
$$

where $\mu_{k}$ is the intercept, $f_{j}^{k}$ is the non-parametric function at the $k$-th quantile level, and $k=1, \ldots, K$. We assume that each $X_{i j}$ takes value 
in $[0,1]$ without loss of generality. To ensure the identifiability of $f_{j}^{k}$,s, we assume that $\mathrm{E} f_{j}^{k}\left(X_{i j}\right)=0$.

\subsection{Proposed Methodologies}

We use polynomial splines to approximate the non-parametric components in model (2.1). Let $0=t_{0}<t_{1}<\cdots<t_{k_{n}}<t_{k_{n}+1}=1$ be a partition of $[0,1]$ into subintervals $\left[t_{l}, t_{l+1}\right), l=0, \ldots, k_{n}$, with $k_{n}$ internal knots. We only focus on equally spaced knots for simplicity. There exists a normalized B-spline basis $\left\{B_{1}, \ldots, B_{m_{n}+1}\right\}$ for the collection of splines of degree $m$, where $m_{n}=k_{n}+m$. Because of the identifiability condition, we restrict our attention to the subspace $\mathcal{S}_{j}^{0}:=\left\{s: s(x)=\sum_{l=1}^{m_{n}+1} B_{l}(x) \beta_{j l}, \sum_{i=1}^{n} s\left(X_{i j}\right)=0\right\}$ with basis $\left\{B_{j l}(x)=B_{l}(x)-\frac{1}{n} \sum_{i=1}^{n} B_{l}\left(X_{i j}\right), l=1, \ldots, m_{n}\right\}$. Using Bsplines expansion, we can approximate each component in model (2.1) by

$$
f_{j}^{k}\left(X_{i j}\right) \approx \sum_{l=1}^{m_{n}} B_{j l}\left(X_{i j}\right) \beta_{j l}^{k}
$$

$j=1, \ldots, p, k=1, \ldots, K$. Let $\boldsymbol{\Pi}_{j}(x)=\left(B_{j 1}(x), \ldots, B_{j m_{n}}(x)\right)^{T}, \boldsymbol{\Pi}\left(\mathbf{X}_{i}\right)=$ $\left(\boldsymbol{\Pi}_{1}\left(X_{i 1}\right)^{T}, \ldots, \boldsymbol{\Pi}_{p}\left(X_{i p}\right)^{T}\right)^{T}$. Then, model (2.1) can be approximated by

$$
Q_{\tau_{k}}\left(\mathbf{X}_{i}\right) \approx \mu_{k}+\Pi\left(\mathbf{X}_{i}\right)^{T} \boldsymbol{\beta}^{k}
$$

where $\boldsymbol{\beta}^{k}=\left(\boldsymbol{\beta}_{1}^{k T}, \ldots, \boldsymbol{\beta}_{p}^{k T}\right)^{T}, \boldsymbol{\beta}_{j}^{k}=\left(\boldsymbol{\beta}_{j 1}^{k}, \ldots, \boldsymbol{\beta}_{j m_{n}}^{k}\right)^{T}, j=1, \ldots, p, k=$ $1, \ldots, K$.

\subsubsection{Standard Quantile Regression Estimator}

We first present standard quantile regression for model (2.1) using polynomial splines. At a given quantile level $\tau_{k}$, the objective function of the 
standard quantile regression with B-spline expansion is

$$
\sum_{i=1}^{n} \rho_{\tau_{k}}\left(Y_{i}-\mu_{k}-\boldsymbol{\Pi}\left(\mathbf{X}_{i}\right)^{T} \boldsymbol{\beta}^{k}\right)
$$

where $\rho_{\tau_{k}}(u)=u\left(\tau_{k}-I(u<0)\right)$ is the quantile check loss function and $I(\cdot)$ is the indicator function. If we consider regression at multiple quantile levels simultaneously, the objective function is

$$
\sum_{k=1}^{K} \sum_{i=1}^{n} \rho_{\tau_{k}}\left(Y_{i}-\mu_{k}-\mathbf{\Pi}\left(\mathbf{X}_{i}\right)^{T} \boldsymbol{\beta}^{k}\right)
$$

which is the same as performing quantile regression at each quantile level separately. The standard quantile estimator for $\mu_{k}, \boldsymbol{\beta}^{k}$ is defined as

$$
\left(\bar{\mu}_{k}, \overline{\boldsymbol{\beta}}^{k}\right)=\underset{\mu_{k} \in \mathbb{R}, \boldsymbol{\beta}^{k} \in \mathbb{R}^{p m_{n}}}{\arg \min } \sum_{k=1}^{K} \sum_{i=1}^{n} \rho_{\tau_{k}}\left(Y_{i}-\mu_{k}-\Pi\left(\mathbf{X}_{i}\right)^{T} \boldsymbol{\beta}^{k}\right) .
$$

Let $\mathcal{H}_{d}$ be the collection of all functions on $[0,1]$ whose $r$-th order derivative satisfies the Hölder condition of order $\alpha$ with $d=r+\alpha>\frac{1}{2}$ and $\alpha \in(0,1]$. That is,

$$
\left|f^{(r)}(s)-f^{(r)}(t)\right| \leq C_{0}|s-t|^{\alpha}, \text { for } s, t \in[0,1]
$$

where $C_{0}$ is a positive constant. The following three conditions are assumed for the theoretical results, where we use subscript 0 to indicate the true value of the intercept and non-parametric functions.

(A1) $\quad \mathrm{E} f_{0 j}^{k}\left(X_{i j}\right)=0$ and $f_{0 j}^{k} \in \mathcal{H}_{d}, j=1,2, \ldots, p, k=1, \ldots, K, d>\frac{1}{2}$.

(A2) Let $\epsilon_{i k}:=Y_{i}-\mu_{0 k}-\sum_{j=1}^{p} f_{0 j}^{k}\left(X_{i j}\right), i=1, \ldots, n, k=1, \ldots, K$. We have $\mathrm{P}\left\{\epsilon_{i k} \leq 0 \mid \mathbf{X}\right\}=\tau_{k}$. The conditional distribution of $\epsilon_{i k}$ given $\left\{X_{i j}\right\}$ has a density function $f_{\epsilon_{i}}^{k}$ with $0<C_{1}<f_{\epsilon_{i}}^{k}(0)<C_{2}<\infty$ for some positive constants $C_{1}, C_{2}$. The density function $f_{\epsilon_{i}}^{k}$ has a bounded first derivative in a neighborhood of zero, uniformly over $i$. 
(A3) $\left(\mathbf{X}_{1}, \ldots, \mathbf{X}_{p}\right)$ has a continuous density on $[0,1]^{p}$. The marginal density of $\mathbf{X}_{j}$ is bounded and bounded away from zero.

\subsubsection{Penalized Estimator FAGL1}

In this section, we present the penalized estimator FAGL1 to investigate interquantile shrinkage of the non-parametric components in model (2.1). To simplify presentation and theoretical derivations, we reparameterize the model as follows. Denote by $\mathbf{d}_{j}^{k}=\boldsymbol{\beta}_{j}^{k}-\boldsymbol{\beta}_{j}^{k-1}$ the coefficient difference at two neighboring quantiles $\tau_{k-1}$ and $\tau_{k}$, with $k=2, \ldots, K$ and $\mathbf{d}_{j}^{1}=\boldsymbol{\beta}_{j}^{1}$ for $j=1, \ldots, p$. Let $\boldsymbol{\theta}=\left(\boldsymbol{\mu}^{T}, \mathbf{d}^{1 T}, \ldots, \mathbf{d}^{K T}\right)^{T} \in \mathbb{R}^{K+K p m_{n}}$ be the collection of unknown parameters, where $\boldsymbol{\mu}=\left(\mu_{1}, \ldots, \mu_{K}\right)^{T}, \mathbf{d}^{k}=\left(\mathbf{d}_{1}^{k T}, \ldots, \mathbf{d}_{p}^{k T}\right)^{T}$ for $k=1, \ldots, K$. Let $\boldsymbol{\theta}_{0}$ be the true value of $\boldsymbol{\theta}$.

By Corollary 6.21 of Schumaker (2007), under condition (A1), there exists a positive constant $C$ which depends on $m, C_{0}$, and $\boldsymbol{\beta}_{0 j}^{k}$, such that $\sup _{x \in[0,1]}\left|r_{j}^{k}(x)\right| \leq C k_{n}^{-d}$, where $r_{j}^{k}(x)=\boldsymbol{\Pi}_{j}(x)^{T} \boldsymbol{\beta}_{0 j}^{k}-f_{0 j}^{k}(x)$ and $\boldsymbol{\beta}_{0 j}^{k}$ is the true value of $\boldsymbol{\beta}_{j}^{k}$. Then, the objective function 2.2 can be written as

$$
\sum_{k=1}^{K} \sum_{i=1}^{n} \rho_{\tau_{k}}\left(\epsilon_{i k}-R^{k}\left(\mathbf{X}_{i}\right)-\left(\mu_{k}-\mu_{0 k}\right)-\boldsymbol{\Pi}\left(\mathbf{X}_{i}\right)^{T}\left(\boldsymbol{\beta}^{k}-\boldsymbol{\beta}_{0}^{k}\right)\right),
$$

where $R^{k}\left(\mathbf{X}_{i}\right)=\sum_{j=1}^{p} r_{j}^{k}\left(X_{i j}\right)$, and we have

$$
\begin{aligned}
& \sum_{k=1}^{K} \sum_{i=1}^{n} \rho_{\tau_{k}}\left(\epsilon_{i k}-R^{k}\left(\mathbf{X}_{i}\right)-\left(1, \Pi\left(\mathbf{X}_{i}\right)^{T}\right)\left(\mu_{k}-\mu_{0 k},\left(\boldsymbol{\beta}^{k}-\boldsymbol{\beta}_{0}^{k}\right)^{T}\right)^{T}\right) \\
= & \sum_{k=1}^{K} \sum_{i=1}^{n} \rho_{\tau_{k}}\left(\epsilon_{i k}-R^{k}\left(\mathbf{X}_{i}\right)-\left(1, \Pi\left(\mathbf{X}_{i}\right)^{T}\right) \mathbf{T}_{k}\left(\boldsymbol{\theta}-\boldsymbol{\theta}_{0}\right)\right) \\
= & \sum_{k=1}^{K} \sum_{i=1}^{n} \rho_{\tau_{k}}\left(\epsilon_{i k}-R^{k}\left(\mathbf{X}_{i}\right)-\mathbf{z}_{i k}^{T}\left(\boldsymbol{\theta}-\boldsymbol{\theta}_{0}\right)\right),
\end{aligned}
$$

where $\mathbf{z}_{i k}^{T}=\left(1, \boldsymbol{\Pi}\left(\mathbf{X}_{i}\right)^{T}\right) \mathbf{T}_{k}$, and the matrix $\mathbf{T}_{k}$ is defined through $\mathbf{T}_{k}(\boldsymbol{\theta}-$ $\left.\boldsymbol{\theta}_{0}\right)=\left(\mu_{k}-\mu_{0 k},\left(\boldsymbol{\beta}^{k}-\boldsymbol{\beta}_{0}^{k}\right)^{T}\right)^{T}$. More concretely, $\mathbf{T}_{k}=\left(\mathbf{D}_{k, 1}, \mathbf{D}_{k, 2}, \mathbf{D}_{k, 3}\right) \in$ 
$\mathbb{R}^{\left(1+p m_{n}\right) \times\left(K+K p m_{n}\right)}$, where $\mathbf{D}_{k, 1}$ is a $\left(1+p m_{n}\right) \times K$ matrix with its $(1, k)$ entry being 1 and other entries being 0 , that is

$$
\mathbf{D}_{k, 1}=\left(\begin{array}{ccccc}
0 & \cdots & 1 & \cdots & 0 \\
0 & \cdots & 0 & \cdots & 0 \\
\cdots & \cdots & \cdots & \cdots & \cdots \\
0 & \cdots & 0 & \cdots & 0
\end{array}\right)_{\left(1+p m_{n}\right) \times K}
$$

$\mathbf{D}_{k, 2}$ is a $\left(1+p m_{n}\right) \times k p m_{n}$ matrix with $\left(\mathbf{0}_{p m_{n}}, \boldsymbol{I}_{p m_{n}}\right)^{T}$ repeated $k$ times, where $\mathbf{0}_{p m_{n}} \in \mathbb{R}^{p m_{n}}$ is a zero vector and $\boldsymbol{I}_{p m_{n}}$ is the identity matrix of dimension $p m_{n}$, that is

$$
\mathbf{D}_{k, 2}=\left(\begin{array}{cccccccccc}
0 & 0 & \cdots & 0 & \cdots & \cdots & 0 & 0 & \cdots & 0 \\
1 & 0 & \cdots & 0 & \cdots & \cdots & 1 & 0 & \cdots & 0 \\
0 & 1 & \cdots & 0 & \cdots & \cdots & 0 & 1 & \cdots & 0 \\
\cdots & \cdots & \cdots & \cdots & \cdots & \cdots & 0 & 0 & \cdots & 0 \\
0 & 0 & \cdots & 1 & \cdots & \cdots & 0 & 0 & \cdots & 1
\end{array}\right)_{\left(1+p m_{n}\right) \times k p m_{n}}
$$

and $\mathbf{D}_{k, 3}$ is a $\left(1+p m_{n}\right) \times(K-k) p m_{n}$ zero matrix.

The FAGL1 estimator is defined as

$$
\hat{\boldsymbol{\theta}}=\underset{\boldsymbol{\theta} \in \mathbb{R}^{K+K p m_{n}}}{\arg \min } Q_{1}(\boldsymbol{\theta})
$$

where

$Q_{1}(\boldsymbol{\theta})=\sum_{k=1}^{K} \sum_{i=1}^{n} \rho_{\tau_{k}}\left(\epsilon_{i k}-R^{k}\left(\mathbf{X}_{i}\right)-\mathbf{z}_{i k}^{T}\left(\boldsymbol{\theta}-\boldsymbol{\theta}_{0}\right)\right)+\lambda_{n} \sum_{k=2}^{K} \sum_{j=1}^{p} \omega_{j, k}\left\|\mathbf{d}_{j}^{k}\right\|$.

Here, $\lambda_{n} \geq 0$ is a tuning parameter controlling the degree of penalization, $\omega_{j, k}$ is the weight for $\mathbf{d}_{j}^{k}$. Note that $\mathbf{d}_{j}^{k}$ is a group of parameters corresponding to the differences of the $(k-1)$-th and $k$-th quantile level of the $j$-th non-parametric component $f_{j}$. Let $\overline{\boldsymbol{\theta}}$ be the initial estimator obtained from 
standard quantile regression (2.3). Following Zou (2006), we set the weights $\omega_{j, k}=\left\|\overline{\mathbf{d}}_{j}^{k}\right\|^{-1}$. Since the penalty is performed in a group manner, either all or none of the elements in $\mathbf{d}_{j}^{k}$ will be shrunk to 0 . If $\mathbf{d}_{j}^{k}$ is shrunk to 0 , this implies the non-parametric component $f_{j}$ remain the same for the $(k-1)$-th and the $k$-th quantile levels. By employing this penalty, we can identify the quantile regions where each component $f_{j}$ varies or remains unvarying.

\subsubsection{Penalized Estimator FAGL2}

In this section, we present another penalty which penalizes all component functions associated with each predictor as one group. The penalized estimator FAGL2 is defined as (with abuse of notation)

$$
\hat{\boldsymbol{\theta}}=\underset{\boldsymbol{\theta} \in \mathbb{R}^{K+K p m_{n}}}{\arg \min } Q_{2}(\boldsymbol{\theta}),
$$

where

$$
Q_{2}(\boldsymbol{\theta})=\sum_{k=1}^{K} \sum_{i=1}^{n} \rho_{\tau_{k}}\left(\epsilon_{i k}-R^{k}\left(\mathbf{X}_{i}\right)-\mathbf{z}_{i k}^{T}\left(\boldsymbol{\theta}-\boldsymbol{\theta}_{0}\right)\right)+\lambda_{n} \sum_{j=1}^{p} \omega_{j}\left\|\boldsymbol{\theta}_{(j)}\right\| .
$$

Here, $\boldsymbol{\theta}_{(j)}=\left(\mathbf{d}_{j}^{2 T}, \ldots, \mathbf{d}_{j}^{K T}\right)^{T}$ and $\omega_{j}$ is the weight for $\boldsymbol{\theta}_{(j)}$. Letting $\overline{\boldsymbol{\theta}}$ be the initial estimator obtained from standard quantile regression (2.3), we set $\omega_{j}=\left\|\overline{\boldsymbol{\theta}}_{(j)}\right\|^{-1}$. In this case, we penalize the interquantile difference $\boldsymbol{\theta}_{(j)}$ of each non-parametric component $f_{j}$ as one group. That is, we expect some functions are unvarying across all quantile levels.

\subsubsection{Computation}

Here we discuss the algorithm we use to solve the optimization problems for the proposed methods. We adopt the majorize-minimize (MM) algorithm proposed by Hunter and Lange (2000) to deal with the unsmooth check loss 
function. More specifically, we use the majorization function

$$
\phi_{x^{(t)}}^{k}(x)=\frac{1}{4}\left[\frac{x^{2}}{\epsilon+\left|x^{(t)}\right|}+\left(4 \tau_{k}-2\right) x+c\right]
$$

to replace the non-differentiable quantile check loss function $\rho_{\tau_{k}}(x)$, where $x^{(t)}=Y_{i}-\mathbf{z}_{i k}^{T} \boldsymbol{\theta}^{(t)}, \boldsymbol{\theta}^{(t)}$ is the value of $\boldsymbol{\theta}$ at the $t$-th iteration, $t=1,2, \ldots$ and $\epsilon$ is a small constant to make sure the denominator in 2.4 is not equal to zero, and $c$ is a constant that does not involve $\boldsymbol{\theta}$. In each iteration, we use the $\mathrm{R}$ function gglasso in the package gglasso to solve the objective function with $\rho_{\tau_{k}}(x)$ replaced by $\phi_{x^{(t)}}^{k}(x)$.

As for the degree of the splines, we use cubic splines $(m=3)$ which is most frequently used in statistical literature. For the number of internal knots $k_{n}$, we use the Schwarz information criterion (SIC, Schwarz (1978)) defined as

$$
S I C\left(k_{n}\right)=\sum_{k=1}^{K} \log \left[\sum_{i=1}^{n} \rho_{\tau_{k}}\left(Y_{i}-\mathbf{z}_{i k}^{T} \overline{\boldsymbol{\theta}}\right)\right]+\frac{\log (n)}{2 n} K p\left(k_{n}+m\right)
$$

where $\overline{\boldsymbol{\theta}}$ is the standard quantile estimator obtained using $k_{n}$ knots without penalization. We select the number of knots that minimizes the SIC.

For FAGL1 and FAGL2, we use the same number of knots as in standard quantile regression, and select the tuning parameter $\lambda_{n}$ again by minimizing the SIC, which is defined as

$$
S I C\left(\lambda_{n}\right)=\sum_{k=1}^{K} \log \left[\sum_{i=1}^{n} \rho_{\tau_{k}}\left(Y_{i}-\mathbf{z}_{i k}^{T} \hat{\boldsymbol{\theta}}\right)\right]+\frac{\log (n)}{2 n} d f
$$

where $\hat{\boldsymbol{\theta}}$ is the penalized estimator FAGL1 or FAGL2 obtained given $\lambda_{n}$ and the degrees of freedom $d f$ is the number of nonzero components in $\hat{\boldsymbol{\theta}}$. 


\section{$2.2 \quad$ Theoretical Results}

\subsubsection{Standard Quantile Regression Estimator}

We denote by $\bar{f}_{j}^{k}$ the quantile estimator of non-parametric component $f_{j}^{k}$ defined through $\overline{\boldsymbol{\beta}}_{j}^{k}$. The following result on the convergence rate of $\bar{f}_{j}^{k}$ is well known (He and Shi (1994): Kim (2007)) and is produced here mainly for completeness. In the statement of the theorem, we use $a_{n} \sim b_{n}$ to mean that there are constants $0<A<B<\infty$ such that $A \leq a_{n} / b_{n} \leq B$ for all sufficiently large $n$.

Theorem 2.1. Assume conditions (A1) - (A3) hold and $k_{n} \sim n^{\frac{1}{2 d+1}}$. Then for $j=1,2, \ldots, p$,

$$
\frac{1}{n} \sum_{i=1}^{n}\left(\bar{f}_{j}^{k(l)}\left(X_{i j}\right)-f_{0 j}^{k(l)}\left(X_{i j}\right)\right)^{2}=O_{p}\left(n^{-\frac{2(d-l)}{2 d+1}}\right),
$$

where $f^{(l)}$ denotes the $l$-th order derivative of $f, l=0,1, \ldots, m$.

Since we define the quantile estimate $\bar{f}_{j}^{k}$ through $\overline{\boldsymbol{\beta}}_{j}^{k}$ and B-spline basis functions, the $l$-th order derivative of $\bar{f}_{j}^{k}$ can be obtained through $\overline{\boldsymbol{\beta}}_{j}^{k}$ and the $l$-th derivative of B-spline basis functions.

\subsubsection{Penalized Estimator FAGL1}

Now we consider the theoretical properties of FAGL1. Let $\mathcal{A}=\{(j, k)$ : $\left.\left\|f_{0 j}^{k}-f_{0 j}^{k-1}\right\|=0, j=1, \ldots, p, k=2, \ldots, K\right\}$ in the true model, where we use $\|$. $\|$ to denote the $L_{2}$ norm for squared integral functions on $[0,1]$. Each nonparametric component $f_{j}$ may vary in different quantile regions. However, for simplicity of notation we assume that all the non-parametric components vary across the first $k_{0}$ quantile levels. This means $\left\|f_{0 j}^{k}-f_{0 j}^{k-1}\right\|=0$ for 
$k=k_{0}+1, \ldots, K, j=1, \ldots, p$. Denote by $\hat{f}_{j}^{k}$ the penalized quantile estimator of $f_{j}^{k}$.

Theorem 2.2. (Estimation Rate) Assume conditions (A1) - (A3) hold and $k_{n} \sim n^{\frac{1}{2 d+1}}$. If $\frac{\lambda_{n}}{n} \rightarrow 0$, then

$$
\frac{1}{n} \sum_{i=1}^{n}\left(\hat{f}_{j}^{k(l)}\left(X_{i j}\right)-f_{0 j}^{k(l)}\left(X_{i j}\right)\right)^{2}=O_{p}\left(n^{-\frac{2(d-l)}{2 d+1}}\right),
$$

where $f^{(l)}$ denotes the $l$-th order derivative of $f, j=1, \ldots, p, l=0,1, \ldots, m$.

Theorem 2.3. (Selection Consistency) Assume conditions (A1) - (A3) hold and $k_{n} \sim n^{\frac{1}{2 d+1}}$. If $\frac{\lambda_{n}}{n} \rightarrow 0$ and $\frac{\lambda_{n}}{\sqrt{n k_{n}}} \rightarrow \infty$, then

$$
\mathrm{P}\left(\left\{(j, k):\left\|\hat{\mathbf{d}}_{j}^{k}\right\|=0, j=1, \ldots, p, k=2, \ldots, K\right\}=\mathcal{A}\right) \rightarrow 1 .
$$

\subsubsection{Penalized Estimator FAGL2}

Let $\mathcal{B}=\left\{j:\left\|f_{0 j}^{k}-f_{0 j}^{k-1}\right\|=0\right.$, for all $\left.k=2, \ldots, K\right\}$. Without loss of generality, we assume that the non-parametric component functions vary across quantile levels for the first $q$ predictors and unvarying across all quantile levels for other predictors. That is, $\left\|f_{0 j}^{k}-f_{0 j}^{k-1}\right\|=0$ for $j=$ $q+1, \ldots, p, k=2, \ldots K$. Let $\hat{f}_{j}^{k}$ be the penalized quantile estimator of $f_{j}^{k}$. Theorem 2.4. (Estimation Rate) Assume conditions (A1) - (A3) hold and $k_{n} \sim n^{\frac{1}{2 d+1}}$. If $\frac{\lambda_{n}}{n} \rightarrow 0$, then

$$
\frac{1}{n} \sum_{i=1}^{n}\left(\hat{f}_{j}^{k(l)}\left(X_{i j}\right)-f_{0 j}^{k(l)}\left(X_{i j}\right)\right)^{2}=O_{p}\left(n^{-\frac{2(d-l)}{2 d+1}}\right),
$$

where $f^{(l)}$ denotes the $l$-th order derivative of $f, j=1, \ldots, p, l=0,1, \ldots, m$.

Theorem 2.5. (Selection Consistency) Assume conditions (A1) - (A3) hold and $k_{n} \sim n^{\frac{1}{2 d+1}}$. If $\frac{\lambda_{n}}{n} \rightarrow 0$ and $\frac{\lambda_{n}}{\sqrt{n k_{n}}} \rightarrow \infty$, then

$$
\mathrm{P}\left(\left\{j:\left\|\hat{\boldsymbol{\theta}}_{(j)}\right\|=0, j=1, \ldots, p\right\}=\mathcal{B}\right) \rightarrow 1 .
$$




\section{$2.3 \quad$ Numerical Results}

\subsubsection{Simulation Study}

In this section, we employ the proposed penalized methods in simulation studies. In the following examples, simulations are repeated 100 times with 9 quantile levels $\{0.1,0.2, \ldots, 0.9\}$. We compare FAGL1, FAGL2 and the standard quantile regression method (QR).

The performances of various methods are evaluated by three measures. The first measure is the integrated squared error (ISE) for each non-parametric function $f_{j}^{k}$, which is approximated by

$$
\widehat{I S E}_{j}^{k}=\frac{1}{T} \sum_{i=1}^{T}\left(\hat{f}_{j}^{k}\left(t_{i}\right)-f_{0 j}^{k}\left(t_{i}\right)\right)^{2}
$$

over an equally spaced 1000 points $\left\{t_{1}, \ldots, t_{T}\right\}, T=1000$ on $[0,1]$. The second measure is true proportion of correct selection defined as the proportion of times where the interquantile difference $f_{j}^{k}-f_{j}^{k-1}$ is estimated correctly as zero or nonzero. The third measure is prediction error at the $k$-th quantile level $\tau_{k}\left(P E_{k}\right)$ defined as

$$
P E_{k}=\frac{1}{n^{\prime}} \sum_{i=1}^{n^{\prime}} \rho_{\tau_{k}}\left(Y_{i}-\hat{Y}_{i, k}\right)
$$

where $Y_{i}, i=1, \ldots, n^{\prime}=10000$ are the responses of independently generated test samples $\left(Y_{i}, X_{i}\right), i=1, \ldots, n^{\prime}$, and $\hat{Y}_{i, k}, i=1, \ldots, n^{\prime}$ are the predicted values at the $k$-th quantile level using one of the three estimators.

Example 2.1. The data $\left\{\left(\mathbf{X}_{i}, Y_{i}\right), i=1, \ldots, n\right\}$ are generated from

$$
Y_{i}=\mu+\sum_{j=1}^{p} f_{j}\left(X_{i j}\right)+\left(0.5+\left|X_{i 3}\right|\right) \epsilon_{i}, \quad i=1, \ldots, n
$$


where $\mu=1, p=3, f_{1}(x)=5 x, f_{2}(x)=3(2 x-1)^{2}$ and $f_{3}(x)=$ $4 \sin (2 \pi x) /(2-\sin (2 \pi x))$. The covariates $X_{i j}$ are generated from the standard normal distribution with correlations given by $\operatorname{Cov}=\left(X_{i j_{1}}, X_{i j_{2}}\right)=$ $(1 / 2)^{\left|j_{1}-j_{2}\right|}, 1 \leq j_{1}, j_{2} \leq 3$ and then transformed to be marginally uniform on $[0,1]$ by applying the cumulative distribution function of the standard normal distribution. Under model (2.5), the $\tau_{k}$-th conditional quantile of $Y_{i}$ given $\mathbf{X}_{i}$ is $Q_{\tau_{k}}\left(\mathbf{X}_{i}\right)=\mu_{k}+f_{1}\left(X_{i 1}\right)+f_{2}\left(X_{i 2}\right)+f_{3}^{k}\left(X_{i 3}\right)$, where $\mu_{k}=\mu+0.5 F_{\epsilon_{i}}^{-1}\left(\tau_{k}\right), f_{3}^{k}(x)=f_{3}(x)+|x| F_{\epsilon_{i}}^{-1}\left(\tau_{k}\right)$ and $F_{\epsilon_{i}}^{-1}\left(\tau_{k}\right)$ is the $\tau_{k}$-th quantile of the distribution of $\epsilon_{i}$. In this example, we consider different sample sizes $n=100,200$ and two different distributions of $\epsilon_{i}$, including a normal distribution with mean 0 and standard deviation 0.5 and a Student's $\mathrm{t}$ distribution with scale parameter $1 / 3$ and degrees of freedom 3 .

Tables 2.1, 2.4, 2.7 and 2.10 show the ISE of each non-parametric function in Example 2.1. As we expect, the two proposed fused adaptive group LASSO methods, FAGL1 and FAGL2, yield smaller $\sqrt{\widehat{I S E}}$ than QR when the non-parametric function is unvarying $\left(f_{1}\right.$ and $\left.f_{2}\right)$. Unlike QR, the methods we proposed are able to identify which functions in model (2.5) are unvarying functions. Thus, the proposed methods yield higher estimation accuracy especially when the true function is an unvarying function. Tables $2.2,2.5,2.8$ and 2.11 report true proportion of correct selection for proposed methods. The FAGL1 has worse selection accuracy (lower true proportion) than the FAGL2. This is as expected since $f_{1}, f_{2}$ are not varying and $f_{3}$ is varying for all quantile levels. Tables 2.3, 2.6, 2.9 and 2.12 illustrate prediction errors for different methods. The proposed two methods yield similar prediction errors to QR.

We have also carried out simulations for another example where a function is varying on some, not all, of the quantile levels. 
Table 2.1: Summary of $\sqrt{\widehat{I S E}}$ for non-parametric functions $f_{1}, f_{2}$ and $f_{3}$ in Example 2.1 when $n=100$ and $\epsilon_{i}$ follows a normal distribution with mean 0 and standard deviation 0.5. Standard errors based on simulations are shown in the parentheses.

\begin{tabular}{|c|c|c|c|c|c|c|c|c|c|c|}
\hline & $\tau$ & 0.1 & 0.2 & 0.3 & 0.4 & 0.5 & 0.6 & 0.7 & 0.8 & 0.9 \\
\hline \multirow{6}{*}{$f_{1}$} & FAGL1 & 0.1231 & 0.1255 & 0.1261 & 0.1267 & 0.1274 & 0.1289 & 0.1303 & 0.1306 & 0.1306 \\
\hline & & $(0.0465)$ & $(0.0453)$ & $(0.0453)$ & $(0.0452)$ & $(0.0451)$ & $(0.0441)$ & $(0.0458)$ & $(0.0454)$ & $(0.0454)$ \\
\hline & FAGL2 & 0.1277 & 0.1279 & 0.1281 & 0.1282 & 0.1281 & 0.1280 & 0.1281 & 0.1280 & 0.1282 \\
\hline & & $(0.0459)$ & $(0.0458)$ & $(0.0458)$ & $(0.0458)$ & $(0.0458)$ & $(0.0459)$ & $(0.0460)$ & $(0.0460)$ & $(0.0460)$ \\
\hline & QR & 0.2255 & 0.1869 & 0.1734 & 0.1693 & 0.1672 & 0.1647 & 0.1732 & 0.1843 & 0.2221 \\
\hline & & $(0.0811)$ & $(0.0594)$ & $(0.0488)$ & $(0.0570)$ & $(0.0581)$ & $(0.0491)$ & $(0.0561)$ & $(0.0702)$ & $(0.0769)$ \\
\hline \multirow{6}{*}{$f_{2}$} & FAGL1 & 0.1238 & 0.1272 & 0.1277 & 0.1284 & 0.1292 & 0.1306 & 0.1316 & 0.1320 & 0.1322 \\
\hline & & $(0.0424)$ & $(0.0429)$ & $(0.0429)$ & $(0.0426)$ & $(0.0428)$ & $(0.0425)$ & $(0.0427)$ & $(0.0424)$ & $(0.0424)$ \\
\hline & FAGL2 & 0.1285 & 0.1293 & 0.1293 & 0.1291 & 0.1289 & 0.1290 & 0.1293 & 0.1295 & 0.1297 \\
\hline & & $(0.0427)$ & $(0.0428)$ & $(0.0429)$ & $(0.0429)$ & $(0.0427)$ & $(0.0426)$ & $(0.0427)$ & $(0.0428)$ & $(0.0431)$ \\
\hline & QR & 0.2299 & 0.1917 & 0.1694 & 0.1604 & 0.1654 & 0.1666 & 0.1718 & 0.1943 & 0.2259 \\
\hline & & $(0.0755)$ & $(0.0612)$ & $(0.0538)$ & $(0.0492)$ & $(0.0471)$ & $(0.0482)$ & $(0.0579)$ & $(0.0649)$ & $(0.0689)$ \\
\hline \multirow{6}{*}{$f_{3}$} & FAGL1 & 0.6715 & 0.3019 & 0.2347 & 0.1930 & 0.1763 & 0.1870 & 0.2241 & 0.2887 & 0.3955 \\
\hline & & $(0.0378)$ & $(0.0307)$ & $(0.0334)$ & $(0.0385)$ & $(0.0428)$ & $(0.0435)$ & $(0.0415)$ & $(0.0389)$ & $(0.0370)$ \\
\hline & FAGL2 & 0.5814 & 0.3093 & 0.2458 & 0.2083 & 0.1948 & 0.2058 & 0.2410 & 0.3022 & 0.4214 \\
\hline & & $(0.0375)$ & $(0.0351)$ & $(0.0368)$ & $(0.0410)$ & $(0.0457)$ & $(0.0460)$ & $(0.0434)$ & $(0.0414)$ & $(0.0528)$ \\
\hline & QR & 0.4150 & 0.3123 & 0.2444 & 0.2125 & 0.1981 & 0.2165 & 0.2502 & 0.3108 & 0.4124 \\
\hline & & $(0.0454)$ & $(0.0472)$ & $(0.0426)$ & $(0.0519)$ & $(0.0587)$ & $(0.0573)$ & $(0.0520)$ & $(0.0583)$ & $(0.0508)$ \\
\hline
\end{tabular}

Table 2.2: True proportion of correctly identifying the model in Example 2.1 when $n=100$ and $\epsilon_{i}$ follows a normal distribution with mean 0 and standard deviation 0.5 .

\begin{tabular}{ccccccccc}
\hline$f_{1}$ & $\boldsymbol{d}_{1}^{2}=\mathbf{0}$ & $\boldsymbol{d}_{1}^{3}=\mathbf{0}$ & $\boldsymbol{d}_{1}^{4}=\mathbf{0}$ & $\boldsymbol{d}_{1}^{5}=\mathbf{0}$ & $\boldsymbol{d}_{1}^{6}=\mathbf{0}$ & $\boldsymbol{d}_{1}^{7}=\mathbf{0}$ & $\boldsymbol{d}_{1}^{8}=\mathbf{0}$ & $\boldsymbol{d}_{1}^{9}=\mathbf{0}$ \\
\hline FAGL1 & 0.71 & 0.86 & 0.75 & 0.65 & 0.52 & 0.68 & 0.96 & 0.99 \\
FAGL2 & 0.88 & 0.88 & 0.88 & 0.88 & 0.88 & 0.88 & 0.88 & 0.88 \\
\hline$f_{2}$ & $\boldsymbol{d}_{2}^{2}=\mathbf{0}$ & $\boldsymbol{d}_{2}^{3}=\mathbf{0}$ & $\boldsymbol{d}_{2}^{4}=\mathbf{0}$ & $\boldsymbol{d}_{2}^{5}=\mathbf{0}$ & $\boldsymbol{d}_{2}^{6}=\mathbf{0}$ & $\boldsymbol{d}_{2}^{7}=\mathbf{0}$ & $\boldsymbol{d}_{2}^{8}=\mathbf{0}$ & $\boldsymbol{d}_{2}^{9}=\mathbf{0}$ \\
\hline FAGL1 & 0.69 & 0.80 & 0.75 & 0.70 & 0.61 & 0.70 & 0.91 & 0.99 \\
FAGL2 & 0.87 & 0.87 & 0.87 & 0.87 & 0.87 & 0.87 & 0.87 & 0.87 \\
\hline$f_{3}$ & $\boldsymbol{d}_{3}^{2} \neq \mathbf{0}$ & $\boldsymbol{d}_{3}^{3} \neq \mathbf{0}$ & $\boldsymbol{d}_{3}^{4} \neq \mathbf{0}$ & $\boldsymbol{d}_{3}^{5} \neq \mathbf{0}$ & $\boldsymbol{d}_{3}^{6} \neq \mathbf{0}$ & $\boldsymbol{d}_{3}^{7} \neq \mathbf{0}$ & $\boldsymbol{d}_{3}^{8} \neq \mathbf{0}$ & $\boldsymbol{d}_{3}^{9} \neq \mathbf{0}$ \\
\hline FAGL1 & 1.00 & 0.48 & 0.69 & 0.86 & 0.82 & 0.58 & 0.08 & 0.00 \\
FAGL2 & 1.00 & 1.00 & 1.00 & 1.00 & 1.00 & 1.00 & 1.00 & 1.00 \\
\hline \hline
\end{tabular}


Table 2.3: Summary of $P E$ in Example 2.1 when $n=100$ and $\epsilon_{i}$ follows a normal distribution with mean 0 and standard deviation 0.5. Standard errors based on simulations are shown in the parentheses.

\begin{tabular}{cccccccccc}
\hline$\tau$ & 0.1 & 0.2 & 0.3 & 0.4 & 0.5 & 0.6 & 0.7 & 0.8 & 0.9 \\
\hline FAGL1 & 0.1424 & 0.1799 & 0.2198 & 0.2424 & 0.2499 & 0.2430 & 0.2199 & 0.1803 & 0.1237 \\
& $(0.0126)$ & $(0.0230)$ & $(0.0285)$ & $(0.0304)$ & $(0.0306)$ & $(0.0294)$ & $(0.0256)$ & $(0.0205)$ & $(0.0155)$ \\
\hline FAGL2 & 0.1341 & 0.1825 & 0.2231 & 0.2461 & 0.2538 & 0.2465 & 0.2234 & 0.1831 & 0.1245 \\
& $(0.0140)$ & $(0.0233)$ & $(0.0290)$ & $(0.0309)$ & $(0.0310)$ & $(0.0298)$ & $(0.0268)$ & $(0.0224)$ & $(0.0186)$ \\
\hline QR & 0.1428 & 0.1964 & 0.2320 & 0.2532 & 0.2609 & 0.2541 & 0.2323 & 0.1936 & 0.1380 \\
& $(0.0420)$ & $(0.0388)$ & $(0.0337)$ & $(0.0317)$ & $(0.0301)$ & $(0.0307)$ & $(0.0285)$ & $(0.0264)$ & $(0.0293)$ \\
\hline \hline
\end{tabular}

Table 2.4: Summary of $\sqrt{\widehat{I S E}}$ for non-parametric functions $f_{1}, f_{2}$ and $f_{3}$ in Example 2.1 when $n=200$ and $\epsilon_{i}$ follows a normal distribution with mean 0 and standard deviation 0.5. Standard errors based on simulations are shown in the parentheses.

\begin{tabular}{|c|c|c|c|c|c|c|c|c|c|c|}
\hline & $\tau$ & 0.1 & 0.2 & 0.3 & 0.4 & 0.5 & 0.6 & 0.7 & 0.8 & 0.9 \\
\hline \multirow{6}{*}{$f_{1}$} & FAGL1 & 0.0804 & 0.0822 & 0.0827 & 0.0829 & 0.0832 & 0.0839 & 0.0853 & 0.0858 & 0.0858 \\
\hline & & $(0.0303)$ & $(0.0304)$ & $(0.0302)$ & $(0.0302)$ & $(0.0300)$ & $(0.0299)$ & $(0.0300)$ & $(0.0301)$ & $(0.0301)$ \\
\hline & FAGL2 & 0.0825 & 0.0828 & 0.0825 & 0.0825 & 0.0825 & 0.0825 & 0.0825 & 0.0825 & 0.0826 \\
\hline & & $(0.0311)$ & $(0.0311)$ & $(0.0311)$ & $(0.0311)$ & $(0.0311)$ & $(0.0311)$ & $(0.0311)$ & $(0.0311)$ & $(0.0312)$ \\
\hline & QR & 0.1557 & 0.1249 & 0.1115 & 0.1057 & 0.1034 & 0.1051 & 0.1081 & 0.1184 & 0.1514 \\
\hline & & $(0.0471)$ & $(0.0405)$ & $(0.0361)$ & $(0.0313)$ & $(0.0331)$ & $(0.0329)$ & $(0.0363)$ & $(0.0396)$ & $(0.0470)$ \\
\hline \multirow{6}{*}{$f_{2}$} & FAGL1 & 0.0783 & 0.0805 & 0.0807 & 0.0811 & 0.0817 & 0.0820 & 0.0828 & 0.0832 & 0.0832 \\
\hline & & $(0.0297)$ & $(0.0292)$ & $(0.0292)$ & $(0.0291)$ & $(0.0295)$ & $(0.0297)$ & $(0.0300)$ & $(0.0305)$ & $(0.0305)$ \\
\hline & FAGL2 & 0.0802 & 0.0803 & 0.0804 & 0.0805 & 0.0805 & 0.0804 & 0.0804 & 0.0805 & 0.0804 \\
\hline & & $(0.0294)$ & $(0.0292)$ & $(0.0293)$ & $(0.0295)$ & $(0.0294)$ & $(0.0292)$ & $(0.0292)$ & $(0.0295)$ & (0.0298) \\
\hline & $\mathrm{QR}$ & 0.1451 & 0.1260 & 0.1131 & 0.1079 & 0.1066 & 0.1029 & 0.1106 & 0.1218 & 0.1527 \\
\hline & & $(0.0457)$ & $(0.0411)$ & $(0.0367)$ & $(0.0325)$ & $(0.0342)$ & $(0.0362)$ & $(0.0375)$ & $(0.0404)$ & $(0.0553)$ \\
\hline \multirow{6}{*}{$f_{3}$} & FAGL1 & 0.6695 & 0.2680 & 0.1966 & 0.1518 & 0.1353 & 0.1499 & 0.1925 & 0.2632 & 0.3758 \\
\hline & & $(0.0253)$ & $(0.0192)$ & $(0.0192)$ & $(0.0195)$ & $(0.0192)$ & $(0.0167)$ & $(0.0151)$ & $(0.0162)$ & $(0.0183)$ \\
\hline & FAGL2 & 0.5995 & 0.2662 & 0.2013 & 0.1599 & 0.1447 & 0.1582 & 0.1982 & 0.2654 & 0.3847 \\
\hline & & $(0.0274)$ & $(0.0186)$ & $(0.0195)$ & $(0.0221)$ & $(0.0237)$ & $(0.0213)$ & $(0.0182)$ & $(0.0176)$ & $(0.0288)$ \\
\hline & $\mathrm{QR}$ & 0.3700 & 0.2683 & 0.2028 & 0.1646 & 0.1508 & 0.1650 & 0.2014 & 0.2650 & 0.3711 \\
\hline & & $(0.0203)$ & $(0.0211)$ & $(0.0200)$ & $(0.0245)$ & $(0.0263)$ & $(0.0248)$ & $(0.0200)$ & $(0.0192)$ & $(0.0219)$ \\
\hline
\end{tabular}


Table 2.5: True proportion of correctly identifying the model in Example 2.1 when $n=200$ and $\epsilon_{i}$ follows a normal distribution with mean 0 and standard deviation 0.5 .

\begin{tabular}{ccccccccc}
\hline$f_{1}$ & $\boldsymbol{d}_{1}^{2}=\mathbf{0}$ & $\boldsymbol{d}_{1}^{3}=\mathbf{0}$ & $\boldsymbol{d}_{1}^{4}=\mathbf{0}$ & $\boldsymbol{d}_{1}^{5}=\mathbf{0}$ & $\boldsymbol{d}_{1}^{6}=\mathbf{0}$ & $\boldsymbol{d}_{1}^{7}=\mathbf{0}$ & $\boldsymbol{d}_{1}^{8}=\mathbf{0}$ & $\boldsymbol{d}_{1}^{9}=\mathbf{0}$ \\
\hline FAGL1 & 0.68 & 0.87 & 0.82 & 0.71 & 0.54 & 0.55 & 0.86 & 0.99 \\
FAGL2 & 0.89 & 0.89 & 0.89 & 0.89 & 0.89 & 0.89 & 0.89 & 0.89 \\
\hline$f_{2}$ & $\boldsymbol{d}_{2}^{2}=\mathbf{0}$ & $\boldsymbol{d}_{2}^{3}=\mathbf{0}$ & $\boldsymbol{d}_{2}^{4}=\mathbf{0}$ & $\boldsymbol{d}_{2}^{5}=\mathbf{0}$ & $\boldsymbol{d}_{2}^{6}=\mathbf{0}$ & $\boldsymbol{d}_{2}^{7}=\mathbf{0}$ & $\boldsymbol{d}_{2}^{8}=\mathbf{0}$ & $\boldsymbol{d}_{2}^{9}=\mathbf{0}$ \\
\hline FAGL1 & 0.62 & 0.85 & 0.76 & 0.73 & 0.63 & 0.63 & 0.93 & 1.00 \\
FAGL2 & 0.87 & 0.87 & 0.87 & 0.87 & 0.87 & 0.87 & 0.87 & 0.87 \\
\hline$f_{3}$ & $\boldsymbol{d}_{3}^{2} \neq \mathbf{0}$ & $\boldsymbol{d}_{3}^{3} \neq \mathbf{0}$ & $\boldsymbol{d}_{3}^{4} \neq \mathbf{0}$ & $\boldsymbol{d}_{3}^{5} \neq \mathbf{0}$ & $\boldsymbol{d}_{3}^{6} \neq \mathbf{0}$ & $\boldsymbol{d}_{3}^{7} \neq \mathbf{0}$ & $\boldsymbol{d}_{3}^{8} \neq \mathbf{0}$ & $\boldsymbol{d}_{3}^{9} \neq \mathbf{0}$ \\
\hline FAGL1 & 1.00 & 0.59 & 0.84 & 0.98 & 0.97 & 0.83 & 0.26 & 0.01 \\
FAGL2 & 1.00 & 1.00 & 1.00 & 1.00 & 1.00 & 1.00 & 1.00 & 1.00 \\
\hline \hline
\end{tabular}

Table 2.6: Summary of PE in Example 2.1 when $n=200$ and $\epsilon_{i}$ follows a normal distribution with mean 0 and standard deviation 0.5. Standard errors based on simulations are shown in the parentheses.

\begin{tabular}{cccccccccc}
\hline$\tau$ & 0.1 & 0.2 & 0.3 & 0.4 & 0.5 & 0.6 & 0.7 & 0.8 & 0.9 \\
\hline FAGL1 & 0.1355 & 0.1601 & 0.1963 & 0.2186 & 0.2274 & 0.2232 & 0.2054 & 0.1732 & 0.1265 \\
& $(0.0077)$ & $(0.0091)$ & $(0.0116)$ & $(0.0144)$ & $(0.0164)$ & $(0.0172)$ & $(0.0173)$ & $(0.0160)$ & $(0.0123)$ \\
\hline FAGL2 & 0.1296 & 0.1610 & 0.1971 & 0.2190 & 0.2275 & 0.2229 & 0.2045 & 0.1712 & 0.1226 \\
& $(0.0085)$ & $(0.0096)$ & $(0.0115)$ & $(0.0137)$ & $(0.0156)$ & $(0.0163)$ & $(0.0165)$ & $(0.0157)$ & $(0.0135)$ \\
\hline QR & 0.1140 & 0.1696 & 0.2049 & 0.2246 & 0.2305 & 0.2227 & 0.2010 & 0.1641 & 0.1078 \\
& $(0.0162)$ & $(0.0187)$ & $(0.0179)$ & $(0.0161)$ & $(0.0156)$ & $(0.0128)$ & $(0.0098)$ & $(0.0084)$ & $(0.0069)$ \\
\hline \hline
\end{tabular}


Table 2.7: Summary of $\sqrt{\widehat{I S E}}$ for non-parametric functions $f_{1}, f_{2}$ and $f_{3}$ in Example 2.1 when $n=100$ and $\epsilon_{i}$ follows a Student's t distribution with scale parameter $1 / 3$ and degrees of freedom 3. Standard errors based on simulations are shown in the parentheses.

\begin{tabular}{|c|c|c|c|c|c|c|c|c|c|c|}
\hline & $\tau$ & 0.1 & 0.2 & 0.3 & 0.4 & 0.5 & 0.6 & 0.7 & 0.8 & 0.9 \\
\hline \multirow{6}{*}{$f_{1}$} & FAGL1 & 0.1213 & 0.1230 & 0.1234 & 0.1240 & 0.1242 & 0.1242 & 0.1256 & 0.1292 & 0.1316 \\
\hline & & $(0.0420)$ & $(0.0425)$ & $(0.0422)$ & $(0.0419)$ & $(0.0413)$ & $(0.0403)$ & $(0.0409)$ & $(0.0450)$ & $(0.0505)$ \\
\hline & FAGL2 & 0.1269 & 0.1280 & 0.1278 & 0.1273 & 0.1268 & 0.1267 & 0.1266 & 0.1269 & 0.1273 \\
\hline & & $(0.0406)$ & $(0.0419)$ & $(0.0415)$ & $(0.0408)$ & $(0.0405)$ & $(0.0405)$ & $(0.0406)$ & $(0.0409)$ & $(0.0411)$ \\
\hline & $\mathrm{QR}$ & 0.2594 & 0.1846 & 0.1592 & 0.1442 & 0.1438 & 0.1442 & 0.1476 & 0.1708 & 0.2439 \\
\hline & & $(0.1438)$ & $(0.0769)$ & $(0.0654)$ & $(0.0528)$ & $(0.0529)$ & $(0.0432)$ & $(0.0413)$ & $(0.0724)$ & $(0.1130)$ \\
\hline \multirow{6}{*}{$f_{2}$} & FAGL1 & 0.1201 & 0.1221 & 0.1222 & 0.1222 & 0.1224 & 0.1246 & 0.1291 & 0.1311 & 0.1369 \\
\hline & & $(0.0458)$ & $(0.0462)$ & $(0.0461)$ & $(0.0461)$ & $(0.0464)$ & $(0.0482)$ & $(0.0553)$ & $(0.0569)$ & $(0.0661)$ \\
\hline & FAGL2 & 0.1259 & 0.1259 & 0.1260 & 0.1261 & 0.1261 & 0.1260 & 0.1261 & 0.1264 & 0.1264 \\
\hline & & $(0.0462)$ & $(0.0461)$ & $(0.0462)$ & $(0.0462)$ & $(0.0463)$ & $(0.0465)$ & $(0.0468)$ & $(0.0471)$ & $(0.0469)$ \\
\hline & $\mathrm{QR}$ & 0.2527 & 0.1843 & 0.1556 & 0.1406 & 0.1382 & 0.1462 & 0.1603 & 0.1905 & 0.2774 \\
\hline & & $(0.1245)$ & $(0.0708)$ & $(0.0568)$ & $(0.0509)$ & $(0.0560)$ & $(0.0594)$ & $(0.0738)$ & $(0.0804)$ & $(0.1546)$ \\
\hline \multirow{6}{*}{$f_{3}$} & FAGL1 & 0.6507 & 0.2573 & 0.2044 & 0.1753 & 0.1648 & 0.1716 & 0.1974 & 0.2486 & 0.3514 \\
\hline & & $(0.0430)$ & $(0.0354)$ & $(0.0350)$ & $(0.0357)$ & $(0.0364)$ & $(0.0356)$ & $(0.0334)$ & $(0.0334)$ & $(0.0318)$ \\
\hline & FAGL2 & 0.5527 & 0.2861 & 0.2253 & 0.1931 & 0.1826 & 0.1899 & 0.2177 & 0.2768 & 0.4085 \\
\hline & & $(0.0430)$ & $(0.0466)$ & $(0.0416)$ & $(0.0405)$ & $(0.0388)$ & $(0.0378)$ & $(0.0365)$ & $(0.0429)$ & $(0.0709)$ \\
\hline & $\mathrm{QR}$ & 0.4375 & 0.2759 & 0.2136 & 0.1795 & 0.1691 & 0.1781 & 0.2094 & 0.2744 & 0.4109 \\
\hline & & $(0.3216)$ & $(0.0658)$ & $(0.0421)$ & $(0.0403)$ & $(0.0396)$ & $(0.0363)$ & $(0.0360)$ & $(0.0579)$ & $(0.1110)$ \\
\hline
\end{tabular}

Table 2.8: True proportion of correctly identifying the model in Example 2.1 when $n=100$ and $\epsilon_{i}$ follows a Student's t distribution with scale parameter $1 / 3$ and degrees of freedom 3.

\begin{tabular}{ccccccccc}
\hline$f_{1}$ & $\boldsymbol{d}_{1}^{2}=\mathbf{0}$ & $\boldsymbol{d}_{1}^{3}=\mathbf{0}$ & $\boldsymbol{d}_{1}^{4}=\mathbf{0}$ & $\boldsymbol{d}_{1}^{5}=\mathbf{0}$ & $\boldsymbol{d}_{1}^{6}=\mathbf{0}$ & $\boldsymbol{d}_{1}^{7}=\mathbf{0}$ & $\boldsymbol{d}_{1}^{8}=\mathbf{0}$ & $\boldsymbol{d}_{1}^{9}=\mathbf{0}$ \\
\hline FAGL1 & 0.63 & 0.86 & 0.68 & 0.67 & 0.62 & 0.72 & 0.81 & 0.91 \\
FAGL2 & 0.87 & 0.87 & 0.87 & 0.87 & 0.87 & 0.87 & 0.87 & 0.87 \\
\hline$f_{2}$ & $\boldsymbol{d}_{2}^{2}=\mathbf{0}$ & $\boldsymbol{d}_{2}^{3}=\mathbf{0}$ & $\boldsymbol{d}_{2}^{4}=\mathbf{0}$ & $\boldsymbol{d}_{2}^{5}=\mathbf{0}$ & $\boldsymbol{d}_{2}^{6}=\mathbf{0}$ & $\boldsymbol{d}_{2}^{7}=\mathbf{0}$ & $\boldsymbol{d}_{2}^{8}=\mathbf{0}$ & $\boldsymbol{d}_{2}^{9}=\mathbf{0}$ \\
\hline FAGL1 & 0.64 & 0.89 & 0.85 & 0.71 & 0.56 & 0.65 & 0.86 & 0.91 \\
FAGL2 & 0.92 & 0.92 & 0.92 & 0.92 & 0.92 & 0.92 & 0.92 & 0.92 \\
\hline$f_{3}$ & $\boldsymbol{d}_{3}^{2} \neq \mathbf{0}$ & $\boldsymbol{d}_{3}^{3} \neq \mathbf{0}$ & $\boldsymbol{d}_{3}^{4} \neq \mathbf{0}$ & $\boldsymbol{d}_{3}^{5} \neq \mathbf{0}$ & $\boldsymbol{d}_{3}^{6} \neq \mathbf{0}$ & $\boldsymbol{d}_{3}^{7} \neq \mathbf{0}$ & $\boldsymbol{d}_{3}^{8} \neq \mathbf{0}$ & $\boldsymbol{d}_{3}^{9} \neq \mathbf{0}$ \\
\hline FAGL1 & 1.00 & 0.60 & 0.72 & 0.82 & 0.75 & 0.51 & 0.29 & 0.07 \\
FAGL2 & 1.00 & 1.00 & 1.00 & 1.00 & 1.00 & 1.00 & 1.00 & 1.00 \\
\hline \hline
\end{tabular}


Table 2.9: Summary of $P E$ in Example 2.1 when $n=100$ and $\epsilon_{i}$ follows a Student's t distribution with scale parameter $1 / 3$ and degrees of freedom 3 . Standard errors based on simulations are shown in the parentheses.

\begin{tabular}{cccccccccc}
\hline$\tau$ & 0.1 & 0.2 & 0.3 & 0.4 & 0.5 & 0.6 & 0.7 & 0.8 & 0.9 \\
\hline FAGL1 & 0.3893 & 0.4245 & 0.4633 & 0.4838 & 0.4901 & 0.4834 & 0.4631 & 0.4260 & 0.3655 \\
& $(0.0138)$ & $(0.0225)$ & $(0.0304)$ & $(0.0340)$ & $(0.0341)$ & $(0.0320)$ & $(0.0282)$ & $(0.0233)$ & $(0.0189)$ \\
\hline FAGL2 & 0.3781 & 0.4296 & 0.4674 & 0.4875 & 0.4939 & 0.4872 & 0.4669 & 0.4306 & 0.3678 \\
& $(0.0155)$ & $(0.0238)$ & $(0.0302)$ & $(0.0338)$ & $(0.0342)$ & $(0.0318)$ & $(0.0277)$ & $(0.0229)$ & $(0.0186)$ \\
\hline QR & 0.3921 & 0.4446 & 0.4745 & 0.4896 & 0.4944 & 0.4879 & 0.4692 & 0.4388 & 0.3868 \\
& $(0.0426)$ & $(0.0386)$ & $(0.0371)$ & $(0.0349)$ & $(0.0317)$ & $(0.0289)$ & $(0.0272)$ & $(0.0282)$ & $(0.0291)$ \\
\hline \hline
\end{tabular}

Table 2.10: Summary of $\sqrt{\widehat{I S E}}$ for non-parametric functions $f_{1}, f_{2}$ and $f_{3}$ in Example 2.1 when $n=200$ and $\epsilon_{i}$ follows a Student's t distribution with scale parameter $1 / 3$ and degrees of freedom 3. Standard errors based on simulations are shown in the parentheses.

\begin{tabular}{|c|c|c|c|c|c|c|c|c|c|c|}
\hline & $\tau$ & 0.1 & 0.2 & 0.3 & 0.4 & 0.5 & 0.6 & 0.7 & 0.8 & 0.9 \\
\hline \multirow{6}{*}{$f_{1}$} & FAGL1 & 0.0714 & 0.0723 & 0.0725 & 0.0727 & 0.0728 & 0.0736 & 0.0749 & 0.0765 & 0.0787 \\
\hline & & $(0.0240)$ & $(0.0238)$ & $(0.0237)$ & $(0.0236)$ & $(0.0234)$ & $(0.0238)$ & $(0.0240)$ & $(0.0243)$ & $(0.0262)$ \\
\hline & FAGL2 & 0.0733 & 0.0734 & 0.0734 & 0.0733 & 0.0732 & 0.0732 & 0.0733 & 0.0734 & 0.0735 \\
\hline & & $(0.0239)$ & $(0.0241)$ & $(0.0240)$ & $(0.0239)$ & $(0.0238)$ & $(0.0237)$ & $(0.0237)$ & $(0.0237)$ & $(0.0237)$ \\
\hline & QR & 0.1688 & 0.1074 & 0.0884 & 0.0786 & 0.0804 & 0.0832 & 0.0919 & 0.1084 & 0.1580 \\
\hline & & $(0.0670)$ & $(0.0400)$ & $(0.0307)$ & $(0.0275)$ & $(0.0291)$ & $(0.0280)$ & $(0.0272)$ & $(0.0352)$ & $(0.0523)$ \\
\hline \multirow{6}{*}{$f_{2}$} & FAGL1 & 0.0778 & 0.0788 & 0.0788 & 0.0790 & 0.0791 & 0.0793 & 0.0807 & 0.0827 & 0.0862 \\
\hline & & $(0.0268)$ & $(0.0265)$ & $(0.0265)$ & $(0.0266)$ & $(0.0266)$ & $(0.0268)$ & $(0.0279)$ & $(0.0290)$ & $(0.0351)$ \\
\hline & FAGL2 & 0.0800 & 0.0802 & 0.0802 & 0.0802 & 0.0802 & 0.0802 & 0.0802 & 0.0803 & 0.0807 \\
\hline & & $(0.0239)$ & $(0.0241)$ & $(0.0240)$ & $(0.0239)$ & $(0.0238)$ & $(0.0237)$ & $(0.0237)$ & $(0.0237)$ & $(0.0237)$ \\
\hline & $\mathrm{QR}$ & 0.1688 & 0.1187 & 0.0986 & 0.0920 & 0.0863 & 0.0879 & 0.0914 & 0.1106 & 0.1704 \\
\hline & & $(0.0829)$ & $(0.0608)$ & $(0.0331)$ & $(0.0288)$ & $(0.0279)$ & $(0.0270)$ & $(0.0312)$ & $(0.0357)$ & $(0.0783)$ \\
\hline \multirow{6}{*}{$f_{3}$} & FAGL1 & 0.6517 & 0.2266 & 0.1709 & 0.1411 & 0.1306 & 0.1393 & 0.1682 & 0.2229 & 0.3335 \\
\hline & & $(0.0262)$ & $(0.0172)$ & $(0.0167)$ & $(0.0172)$ & $(0.0177)$ & $(0.0172)$ & $(0.0170)$ & $(0.0175)$ & $(0.0182)$ \\
\hline & FAGL2 & 0.5760 & 0.2399 & 0.1818 & 0.1506 & 0.1390 & 0.1464 & 0.1744 & 0.2313 & 0.3556 \\
\hline & & $(0.0255)$ & $(0.0282)$ & $(0.0199)$ & $(0.0195)$ & $(0.0191)$ & $(0.0192)$ & $(0.0198)$ & $(0.0244)$ & $(0.0380)$ \\
\hline & QR & 0.3507 & 0.2275 & 0.1750 & 0.1432 & 0.1338 & 0.1439 & 0.1712 & 0.2251 & 0.3416 \\
\hline & & $(0.0522)$ & $(0.0219)$ & $(0.0227)$ & $(0.0207)$ & $(0.0183)$ & $(0.0213)$ & $(0.0200)$ & $(0.0213)$ & (0.0377) \\
\hline
\end{tabular}


Table 2.11: True proportion of correctly identifying the model in Example 2.1 when $n=200$ and $\epsilon_{i}$ follows a Student's t distribution with scale parameter $1 / 3$ and degrees of freedom 3 .

\begin{tabular}{ccccccccc}
\hline$f_{1}$ & $\boldsymbol{d}_{1}^{2}=\mathbf{0}$ & $\boldsymbol{d}_{1}^{3}=\mathbf{0}$ & $\boldsymbol{d}_{1}^{4}=\mathbf{0}$ & $\boldsymbol{d}_{1}^{5}=\mathbf{0}$ & $\boldsymbol{d}_{1}^{6}=\mathbf{0}$ & $\boldsymbol{d}_{1}^{7}=\mathbf{0}$ & $\boldsymbol{d}_{1}^{8}=\mathbf{0}$ & $\boldsymbol{d}_{1}^{9}=\mathbf{0}$ \\
\hline FAGL1 & 0.71 & 0.86 & 0.80 & 0.79 & 0.66 & 0.69 & 0.81 & 0.88 \\
FAGL2 & 0.87 & 0.87 & 0.87 & 0.87 & 0.87 & 0.87 & 0.87 & 0.87 \\
\hline$f_{2}$ & $\boldsymbol{d}_{2}^{2}=\mathbf{0}$ & $\boldsymbol{d}_{2}^{3}=\mathbf{0}$ & $\boldsymbol{d}_{2}^{4}=\mathbf{0}$ & $\boldsymbol{d}_{2}^{5}=\mathbf{0}$ & $\boldsymbol{d}_{2}^{6}=\mathbf{0}$ & $\boldsymbol{d}_{2}^{7}=\mathbf{0}$ & $\boldsymbol{d}_{2}^{8}=\mathbf{0}$ & $\boldsymbol{d}_{2}^{9}=\mathbf{0}$ \\
\hline FAGL1 & 0.74 & 0.87 & 0.77 & 0.65 & 0.63 & 0.61 & 0.77 & 0.91 \\
FAGL2 & 0.92 & 0.92 & 0.92 & 0.92 & 0.92 & 0.92 & 0.92 & 0.92 \\
\hline$f_{3}$ & $\boldsymbol{d}_{3}^{2} \neq \mathbf{0}$ & $\boldsymbol{d}_{3}^{3} \neq \mathbf{0}$ & $\boldsymbol{d}_{3}^{4} \neq \mathbf{0}$ & $\boldsymbol{d}_{3}^{5} \neq \mathbf{0}$ & $\boldsymbol{d}_{3}^{6} \neq \mathbf{0}$ & $\boldsymbol{d}_{3}^{7} \neq \mathbf{0}$ & $\boldsymbol{d}_{3}^{8} \neq \mathbf{0}$ & $\boldsymbol{d}_{3}^{9} \neq \mathbf{0}$ \\
\hline FAGL1 & 1.00 & 0.59 & 0.79 & 0.92 & 0.88 & 0.65 & 0.32 & 0.08 \\
FAGL2 & 1.00 & 1.00 & 1.00 & 1.00 & 1.00 & 1.00 & 1.00 & 1.00 \\
\hline \hline
\end{tabular}

Table 2.12: Summary of $P E$ in Example 2.1 when $n=200$ and $\epsilon_{i}$ follows a Student's t distribution with scale parameter $1 / 3$ and degrees of freedom 3. Standard errors based on simulations are shown in the parentheses.

\begin{tabular}{cccccccccc}
\hline$\tau$ & 0.1 & 0.2 & 0.3 & 0.4 & 0.5 & 0.6 & 0.7 & 0.8 & 0.9 \\
\hline FAGL1 & 0.3814 & 0.4020 & 0.4367 & 0.4572 & 0.4654 & 0.4622 & 0.4474 & 0.4186 & 0.3695 \\
& $(0.0093)$ & $(0.0105)$ & $(0.0132)$ & $(0.0167)$ & $(0.0190)$ & $(0.0202)$ & $(0.0201)$ & $(0.0182)$ & $(0.0144)$ \\
\hline FAGL2 & 0.3748 & 0.4062 & 0.4392 & 0.4585 & 0.4659 & 0.4622 & 0.4468 & 0.4171 & 0.3650 \\
& $(0.0105)$ & $(0.0118)$ & $(0.0134)$ & $(0.0158)$ & $(0.0177)$ & $(0.0186)$ & $(0.0187)$ & $(0.0177)$ & $(0.0153)$ \\
\hline QR & 0.3529 & 0.4115 & 0.4436 & 0.4595 & 0.4639 & 0.4570 & 0.4375 & 0.4029 & 0.3423 \\
& $(0.0145)$ & $(0.0189)$ & $(0.0190)$ & $(0.0175)$ & $(0.0152)$ & $(0.0127)$ & $(0.0108)$ & $(0.0087)$ & $(0.0092)$ \\
\hline \hline
\end{tabular}


Example 2.2. We consider an example where a function is unvarying across some, but not all, of the 9 quantile levels. Let $f_{1}(x)=\sin (2 \pi x), f_{2}(x)=$ $5 x(1-x), f_{3}(x)=2 x$. The covariates $X_{i j}$ are generated as in example 2.1. We set $Q_{\tau}\left(\mathbf{X}_{i}\right)=\mu(\tau)+f_{1}\left(X_{i 1}\right)+f_{2}^{\tau}\left(X_{i 2}\right)+f_{3}\left(X_{i 3}\right)$ for any $0<\tau<1$, where $\mu(\tau)=F^{-1}(\tau)$,

$$
f_{2}^{\tau}\left(X_{i 2}\right)= \begin{cases}f_{2}\left(X_{i 2}\right), & \text { if } 0<\tau \leq 0.49 \\ f_{2}\left(X_{i 2}\right)+2\left|X_{i 2}\right|\left(F^{-1}(\tau)-F^{-1}(0.49)\right), & \text { if } 0.49<\tau<1\end{cases}
$$

$F^{-1}(\tau)$ is the $\tau$-th quantile of a distribution. To generate data, we first generate a quantile level $u_{i} \sim U(0,1)$ and let $Y_{i}=\mu\left(u_{i}\right)+f_{1}\left(X_{i 1}\right)+f_{2}^{u_{i}}\left(X_{i 2}\right)+$ $f_{3}\left(X_{i 3}\right)$. Note the non-parametric function $f_{2}^{\tau}(x)$ varies among quantiles $\{0.5, \ldots, 0.9\}$, but remain unvarying at quantiles $\{0.1, \ldots, 0.4\}$. Similar to example 2.1, we perform simulations with different sample sizes $n=100,200$ and two different distribution functions $F(\cdot)$, including a normal distribution function with mean 0 and standard deviation 0.5 and a Student's t distribution function with scale parameter $1 / 3$ and degrees of freedom 3 .

Tables 2.13, 2.16, 2.19 and 2.22 report the ISE of each non-parametric function in Example 2.2. The FAGL1 and FAGL2 yield similar or smaller $\sqrt{\widehat{I S E}}$ than QR. From Tables 2.14, 2.17, 2.20 and 2.23, the selection accuracy for this example is not as good as for example 2.1, but the results still often match the true model. Tables 2.15, 2.18, 2.21 and 2.24 illustrate prediction errors for different methods. The proposed two methods yield similar prediction errors to QR.

\subsubsection{Real Data Analysis}

We apply the proposed methods to a $\mathrm{NO}_{2}$ particles data. This dataset results from study where air pollution on a road is related to traffic volume and 
Table 2.13: Summary of $\sqrt{\widehat{I S E}}$ for non-parametric functions $f_{1}, f_{2}$ and $f_{3}$ in Example 2.2 when $n=100$ and $F(\cdot)$ is the normal distribution function with mean 0 and standard deviation 0.5. Standard errors based on simulations are shown in the parentheses.

\begin{tabular}{|c|c|c|c|c|c|c|c|c|c|c|}
\hline & $\tau$ & 0.1 & 0.2 & 0.3 & 0.4 & 0.5 & 0.6 & 0.7 & 0.8 & 0.9 \\
\hline \multirow{6}{*}{$f_{1}$} & FAGL1 & 0.1117 & 0.1117 & 0.1126 & 0.1150 & 0.1208 & 0.1316 & 0.1445 & 0.1537 & 0.1546 \\
\hline & & $(0.0380)$ & $(0.0387)$ & $(0.0390)$ & $(0.0395)$ & $(0.0414)$ & $(0.0454)$ & $(0.0513)$ & $(0.0593)$ & $(0.0602)$ \\
\hline & FAGL2 & 0.1202 & 0.1156 & 0.1160 & 0.1178 & 0.1210 & 0.1251 & 0.1292 & 0.1330 & 0.1318 \\
\hline & & $(0.0408)$ & $(0.0397)$ & $(0.0403)$ & $(0.0407)$ & $(0.0413)$ & $(0.0423)$ & $(0.0446)$ & $(0.0488)$ & $(0.0497)$ \\
\hline & $\mathrm{QR}$ & 0.1183 & 0.1033 & 0.1101 & 0.1288 & 0.1472 & 0.1808 & 0.2093 & 0.2456 & 0.3176 \\
\hline & & $(0.0418)$ & $(0.0366)$ & $(0.0457)$ & $(0.0536)$ & $(0.0603)$ & $(0.0626)$ & $(0.0700)$ & $(0.0960)$ & $(0.1687)$ \\
\hline \multirow{6}{*}{$f_{2}$} & FAGL1 & 0.1217 & 0.1234 & 0.1240 & 0.1270 & 0.1376 & 0.2037 & 0.3233 & 0.4805 & 0.7126 \\
\hline & & $(0.0476)$ & $(0.0473)$ & $(0.0474)$ & $(0.0494)$ & $(0.0565)$ & $(0.0400)$ & $(0.0302)$ & $(0.0274)$ & $(0.0323)$ \\
\hline & FAGL2 & 0.1450 & 0.1317 & 0.1344 & 0.1400 & 0.1457 & 0.1989 & 0.3184 & 0.4806 & 0.7218 \\
\hline & & $(0.0544)$ & $(0.0483)$ & $(0.0500)$ & $(0.0536)$ & $(0.0562)$ & $(0.0350)$ & $(0.0263)$ & $(0.0275)$ & $(0.0325)$ \\
\hline & QR & 0.1174 & 0.1056 & 0.1144 & 0.1379 & 0.1677 & 0.2431 & 0.3643 & 0.5153 & 0.7384 \\
\hline & & $(0.0476)$ & $(0.0417)$ & $(0.0445)$ & $(0.0528)$ & $(0.0630)$ & $(0.0521)$ & $(0.0421)$ & $(0.0458)$ & $(0.0468)$ \\
\hline \multirow{6}{*}{$f_{3}$} & FAGL1 & 0.1037 & 0.1076 & 0.1086 & 0.1110 & 0.1170 & 0.1280 & 0.1415 & 0.1506 & 0.1527 \\
\hline & & $(0.0412)$ & $(0.0410)$ & $(0.0411)$ & $(0.0423)$ & $(0.0450)$ & $(0.0469)$ & $(0.0513)$ & $(0.0566)$ & $(0.0572)$ \\
\hline & FAGL2 & 0.1156 & 0.1120 & 0.1127 & 0.1148 & 0.1180 & 0.1209 & 0.1241 & 0.1270 & 0.1266 \\
\hline & & $(0.0424)$ & $(0.0414)$ & $(0.0414)$ & $(0.0424)$ & $(0.0436)$ & $(0.0440)$ & $(0.0453)$ & $(0.0465)$ & $(0.0467)$ \\
\hline & $\mathrm{QR}$ & 0.1183 & 0.1084 & 0.1126 & 0.1333 & 0.1530 & 0.1736 & 0.2116 & 0.2515 & 0.2859 \\
\hline & & $(0.0488)$ & $(0.0440)$ & $(0.0433)$ & $(0.0536)$ & $(0.0562)$ & $(0.0659)$ & $(0.0711)$ & $(0.0741)$ & $(0.0920)$ \\
\hline
\end{tabular}

Table 2.14: True proportion of correctly identifying the model in Example 2.2 when $n=100$ and $F(\cdot)$ is the normal distribution function with mean 0 and standard deviation 0.5 .

\begin{tabular}{ccccccccc}
\hline$f_{1}$ & $\boldsymbol{d}_{1}^{2}=\mathbf{0}$ & $\boldsymbol{d}_{1}^{3}=\mathbf{0}$ & $\boldsymbol{d}_{1}^{4}=\mathbf{0}$ & $\boldsymbol{d}_{1}^{5}=\mathbf{0}$ & $\boldsymbol{d}_{1}^{6}=\mathbf{0}$ & $\boldsymbol{d}_{1}^{7}=\mathbf{0}$ & $\boldsymbol{d}_{1}^{8}=\mathbf{0}$ & $\boldsymbol{d}_{1}^{9}=\mathbf{0}$ \\
\hline FAGL1 & 0.34 & 0.71 & 0.55 & 0.37 & 0.22 & 0.26 & 0.51 & 0.92 \\
FAGL2 & 0.40 & 0.40 & 0.40 & 0.40 & 0.40 & 0.40 & 0.40 & 0.40 \\
\hline$f_{2}$ & $\boldsymbol{d}_{2}^{2}=\mathbf{0}$ & $\boldsymbol{d}_{2}^{3}=\mathbf{0}$ & $\boldsymbol{d}_{2}^{4}=\mathbf{0}$ & $\boldsymbol{d}_{2}^{5} \neq \mathbf{0}$ & $\boldsymbol{d}_{2}^{6} \neq \mathbf{0}$ & $\boldsymbol{d}_{2}^{7} \neq \mathbf{0}$ & $\boldsymbol{d}_{2}^{8} \neq \mathbf{0}$ & $\boldsymbol{d}_{2}^{9} \neq \mathbf{0}$ \\
\hline FAGL1 & 0.79 & 0.89 & 0.70 & 0.66 & 0.94 & 0.97 & 0.68 & 0.21 \\
FAGL2 & 0.24 & 0.24 & 0.24 & 0.76 & 0.76 & 0.76 & 0.76 & 0.76 \\
\hline$f_{3}$ & $\boldsymbol{d}_{3}^{2}=\mathbf{0}$ & $\boldsymbol{d}_{3}^{3}=\mathbf{0}$ & $\boldsymbol{d}_{3}^{4}=\mathbf{0}$ & $\boldsymbol{d}_{3}^{5}=\mathbf{0}$ & $\boldsymbol{d}_{3}^{6}=\mathbf{0}$ & $\boldsymbol{d}_{3}^{7}=\mathbf{0}$ & $\boldsymbol{d}_{3}^{8}=\mathbf{0}$ & $\boldsymbol{d}_{3}^{9}=\mathbf{0}$ \\
\hline FAGL1 & 0.63 & 0.82 & 0.69 & 0.48 & 0.22 & 0.24 & 0.48 & 0.88 \\
FAGL2 & 0.48 & 0.48 & 0.48 & 0.48 & 0.48 & 0.48 & 0.48 & 0.48 \\
\hline \hline
\end{tabular}


Table 2.15: Summary of $P E$ in Example 2.2 when $n=100$ and $F(\cdot)$ is the normal distribution function with mean 0 and standard deviation 0.5. Standard errors based on simulations are shown in the parentheses.

\begin{tabular}{cccccccccc}
\hline$\tau$ & 0.1 & 0.2 & 0.3 & 0.4 & 0.5 & 0.6 & 0.7 & 0.8 & 0.9 \\
\hline FAGL1 & 0.0973 & 0.1393 & 0.1787 & 0.2091 & 0.2301 & 0.2388 & 0.2301 & 0.1987 & 0.1402 \\
& $(0.0111)$ & $(0.0114)$ & $(0.0109)$ & $(0.0101)$ & $(0.0101)$ & $(0.0103)$ & $(0.0102)$ & $(0.0095)$ & $(0.0088)$ \\
\hline FAGL2 & 0.0941 & 0.1397 & 0.1803 & 0.2113 & 0.2314 & 0.2382 & 0.2287 & 0.1993 & 0.1432 \\
& $(0.0105)$ & $(0.0109)$ & $(0.0112)$ & $(0.0111)$ & $(0.0110)$ & $(0.0109)$ & $(0.0108)$ & $(0.0108)$ & $(0.0112)$ \\
\hline QR & 0.0899 & 0.1376 & 0.1799 & 0.2153 & 0.2398 & 0.2508 & 0.2454 & 0.2173 & 0.1622 \\
& $(0.0127)$ & $(0.0122)$ & $(0.0127)$ & $(0.0125)$ & $(0.0131)$ & $(0.0131)$ & $(0.0130)$ & $(0.0141)$ & $(0.0184)$ \\
\hline \hline
\end{tabular}

Table 2.16: Summary of $\sqrt{\widehat{I S E}}$ for non-parametric functions $f_{1}, f_{2}$ and $f_{3}$ in Example 2.2 when $n=200$ and $F(\cdot)$ is the normal distribution function with mean 0 and standard deviation 0.5. Standard errors based on simulations are shown in the parentheses.

\begin{tabular}{|c|c|c|c|c|c|c|c|c|c|c|}
\hline & $\tau$ & 0.1 & 0.2 & 0.3 & 0.4 & 0.5 & 0.6 & 0.7 & 0.8 & 0.9 \\
\hline \multirow{6}{*}{$f_{1}$} & FAGL1 & 0.0767 & 0.0706 & 0.0707 & 0.0724 & 0.0791 & 0.0867 & 0.0945 & 0.1015 & 0.1062 \\
\hline & & $(0.0202)$ & $(0.0229)$ & $(0.0231)$ & $(0.0240)$ & $(0.0279)$ & $(0.0316)$ & $(0.0361)$ & $(0.0395)$ & $(0.0408)$ \\
\hline & FAGL2 & 0.0784 & 0.0719 & 0.0727 & 0.0757 & 0.0795 & 0.0824 & 0.0849 & 0.0869 & 0.0881 \\
\hline & & $(0.0260)$ & $(0.0236)$ & $(0.0243)$ & $(0.0255)$ & $(0.0276)$ & $(0.0296)$ & $(0.0323)$ & $(0.0331)$ & $(0.0327)$ \\
\hline & QR & 0.0761 & 0.0645 & 0.0658 & 0.0755 & 0.1018 & 0.1194 & 0.1377 & 0.1573 & 0.2048 \\
\hline & & $(0.0238)$ & $(0.0201)$ & $(0.0241)$ & $(0.0257)$ & $(0.0354)$ & $(0.0411)$ & $(0.0510)$ & $(0.0618)$ & $(0.0772)$ \\
\hline \multirow{6}{*}{$f_{2}$} & FAGL1 & 0.0821 & 0.0834 & 0.0837 & 0.0864 & 0.1004 & 0.1776 & 0.3043 & 0.4631 & 0.6979 \\
\hline & & $(0.0307)$ & $(0.0306)$ & $(0.0306)$ & $(0.0327)$ & $(0.0409)$ & $(0.0244)$ & $(0.0185)$ & $(0.0183)$ & $(0.0227)$ \\
\hline & FAGL2 & 0.1063 & 0.0882 & 0.0899 & 0.0983 & 0.1087 & 0.1737 & 0.3010 & 0.4657 & 0.7121 \\
\hline & & $(0.0378)$ & $(0.0317)$ & $(0.0328)$ & $(0.0365)$ & $(0.0403)$ & $(0.0216)$ & $(0.0156)$ & $(0.0172)$ & $(0.0229)$ \\
\hline & QR & 0.0802 & 0.0719 & 0.0702 & 0.0859 & 0.1193 & 0.2032 & 0.3285 & 0.4819 & 0.7033 \\
\hline & & $(0.0244)$ & $(0.0221)$ & $(0.0265)$ & $(0.0377)$ & $(0.0499)$ & $(0.0355)$ & $(0.0314)$ & $(0.0299)$ & $(0.0349)$ \\
\hline \multirow{6}{*}{$f_{3}$} & FAGL1 & 0.0678 & 0.0699 & 0.0706 & 0.0726 & 0.0782 & 0.0876 & 0.0967 & 0.1031 & 0.1070 \\
\hline & & $(0.0248)$ & $(0.0247)$ & $(0.0252)$ & $(0.0262)$ & $(0.0272)$ & $(0.0296)$ & $(0.0340)$ & $(0.0372)$ & $(0.0403)$ \\
\hline & FAGL2 & 0.0771 & 0.0743 & 0.0751 & 0.0773 & 0.0799 & 0.0825 & 0.0840 & 0.0853 & 0.0862 \\
\hline & & $(0.0268)$ & $(0.0254)$ & $(0.0256)$ & $(0.0264)$ & $(0.0273)$ & $(0.0287)$ & $(0.0296)$ & $(0.0309)$ & $(0.0329)$ \\
\hline & $\mathrm{QR}$ & 0.0753 & 0.0661 & 0.0677 & 0.0800 & 0.0977 & 0.1226 & 0.1502 & 0.1637 & 0.2109 \\
\hline & & $(0.0238)$ & $(0.0227)$ & $(0.0242)$ & $(0.0310)$ & $(0.0306)$ & $(0.0341)$ & $(0.0433)$ & $(0.0553)$ & $(0.0661)$ \\
\hline
\end{tabular}


Table 2.17: True proportion of correctly identifying the model in Example 2.2 when $n=200$ and $F(\cdot)$ is the normal distribution function with mean 0 and standard deviation 0.5 .

\begin{tabular}{ccccccccc}
\hline$f_{1}$ & $\boldsymbol{d}_{1}^{2}=\mathbf{0}$ & $\boldsymbol{d}_{1}^{3}=\mathbf{0}$ & $\boldsymbol{d}_{1}^{4}=\mathbf{0}$ & $\boldsymbol{d}_{1}^{5}=\mathbf{0}$ & $\boldsymbol{d}_{1}^{6}=\mathbf{0}$ & $\boldsymbol{d}_{1}^{7}=\mathbf{0}$ & $\boldsymbol{d}_{1}^{8}=\mathbf{0}$ & $\boldsymbol{d}_{1}^{9}=\mathbf{0}$ \\
\hline FAGL1 & 0.17 & 0.72 & 0.52 & 0.23 & 0.19 & 0.24 & 0.40 & 0.75 \\
FAGL2 & 0.23 & 0.23 & 0.23 & 0.23 & 0.23 & 0.23 & 0.23 & 0.23 \\
\hline$f_{2}$ & $\boldsymbol{d}_{2}^{2}=\mathbf{0}$ & $\boldsymbol{d}_{2}^{3}=\mathbf{0}$ & $\boldsymbol{d}_{2}^{4}=\mathbf{0}$ & $\boldsymbol{d}_{2}^{5} \neq \mathbf{0}$ & $\boldsymbol{d}_{2}^{6} \neq \mathbf{0}$ & $\boldsymbol{d}_{2}^{7} \neq \mathbf{0}$ & $\boldsymbol{d}_{2}^{8} \neq \mathbf{0}$ & $\boldsymbol{d}_{2}^{9} \neq \mathbf{0}$ \\
\hline FAGL1 & 0.68 & 0.86 & 0.73 & 0.89 & 0.98 & 0.98 & 0.92 & 0.31 \\
FAGL2 & 0.03 & 0.03 & 0.03 & 0.97 & 0.97 & 0.97 & 0.97 & 0.97 \\
\hline$f_{3}$ & $\boldsymbol{d}_{3}^{2}=\mathbf{0}$ & $\boldsymbol{d}_{3}^{3}=\mathbf{0}$ & $\boldsymbol{d}_{3}^{4}=\mathbf{0}$ & $\boldsymbol{d}_{3}^{5}=\mathbf{0}$ & $\boldsymbol{d}_{3}^{6}=\mathbf{0}$ & $\boldsymbol{d}_{3}^{7}=\mathbf{0}$ & $\boldsymbol{d}_{3}^{8}=\mathbf{0}$ & $\boldsymbol{d}_{3}^{9}=\mathbf{0}$ \\
\hline FAGL1 & 0.59 & 0.75 & 0.58 & 0.35 & 0.21 & 0.26 & 0.49 & 0.75 \\
FAGL2 & 0.36 & 0.36 & 0.36 & 0.36 & 0.36 & 0.36 & 0.36 & 0.36 \\
\hline \hline
\end{tabular}

Table 2.18: Summary of $P E$ in Example 2.2 when $n=200$ and $F(\cdot)$ is the normal distribution function with mean 0 and standard deviation 0.5. Standard errors based on simulations are shown in the parentheses.

\begin{tabular}{cccccccccc}
\hline$\tau$ & 0.1 & 0.2 & 0.3 & 0.4 & 0.5 & 0.6 & 0.7 & 0.8 & 0.9 \\
\hline FAGL1 & 0.0917 & 0.1281 & 0.1648 & 0.1959 & 0.2196 & 0.2301 & 0.2228 & 0.1948 & 0.1431 \\
& $(0.0093)$ & $(0.0071)$ & $(0.0056)$ & $(0.0056)$ & $(0.0066)$ & $(0.0071)$ & $(0.0081)$ & $(0.0088)$ & $(0.0099)$ \\
\hline FAGL2 & 0.0876 & 0.1269 & 0.1657 & 0.1985 & 0.2219 & 0.2316 & 0.2245 & 0.1985 & 0.1487 \\
& $(0.0083)$ & $(0.0060)$ & $(0.0058)$ & $(0.0070)$ & $(0.0078)$ & $(0.0084)$ & $(0.0092)$ & $(0.0098)$ & $(0.0107)$ \\
\hline QR & 0.0744 & 0.1232 & 0.1635 & 0.1967 & 0.2222 & 0.2330 & 0.2260 & 0.1948 & 0.1362 \\
& $(0.0038)$ & $(0.0046)$ & $(0.0057)$ & $(0.0063)$ & $(0.0064)$ & $(0.0059)$ & $(0.0066)$ & $(0.0077)$ & $(0.0093)$ \\
\hline \hline
\end{tabular}


Table 2.19: Summary of $\sqrt{\widehat{I S E}}$ for non-parametric functions $f_{1}, f_{2}$ and $f_{3}$ in Example 2.2 when $n=100$ and $F(\cdot)$ is the Student's t distribution function with scale parameter $1 / 3$ and degrees of freedom 3. Standard errors based on simulations are shown in the parentheses.

\begin{tabular}{|c|c|c|c|c|c|c|c|c|c|c|}
\hline & $\tau$ & 0.1 & 0.2 & 0.3 & 0.4 & 0.5 & 0.6 & 0.7 & 0.8 & 0.9 \\
\hline \multirow{6}{*}{$f_{1}$} & FAGL1 & 0.1018 & 0.1008 & 0.1012 & 0.1023 & 0.1058 & 0.1142 & 0.1352 & 0.2034 & 0.2304 \\
\hline & & $(0.0330)$ & $(0.0335)$ & $(0.0336)$ & $(0.0340)$ & $(0.0353)$ & $(0.0399)$ & $(0.0821)$ & $(0.5690)$ & $(0.5794)$ \\
\hline & FAGL2 & 0.1096 & 0.1056 & 0.1042 & 0.1050 & 0.1075 & 0.1118 & 0.1218 & 0.1709 & 0.1816 \\
\hline & & $(0.0385)$ & $(0.0346)$ & $(0.0348)$ & $(0.0352)$ & $(0.0358)$ & $(0.0381)$ & $(0.0758)$ & $(0.5018)$ & $(0.5528)$ \\
\hline & $\mathrm{QR}$ & 0.1368 & 0.0956 & 0.0910 & 0.1034 & 0.1180 & 0.1436 & 0.1774 & 0.2891 & 0.4394 \\
\hline & & $(0.0588)$ & $(0.0328)$ & $(0.0356)$ & $(0.0410)$ & $(0.0504)$ & $(0.0503)$ & $(0.0673)$ & $(0.5974)$ & $(0.6694)$ \\
\hline \multirow{6}{*}{$f_{2}$} & FAGL1 & 0.1096 & 0.1109 & 0.1111 & 0.1124 & 0.1179 & 0.1621 & 0.2526 & 0.3863 & 0.6189 \\
\hline & & $(0.0415)$ & $(0.0414)$ & $(0.0414)$ & $(0.0420)$ & $(0.0462)$ & $(0.0375)$ & $(0.0327)$ & $(0.0311)$ & $(0.0331)$ \\
\hline & FAGL2 & 0.1273 & 0.1199 & 0.1201 & 0.1227 & 0.1254 & 0.1588 & 0.2455 & 0.3787 & 0.6189 \\
\hline & & $(0.0490)$ & $(0.0433)$ & $(0.0442)$ & $(0.0465)$ & $(0.0482)$ & $(0.0335)$ & $(0.0264)$ & $(0.0246)$ & $(0.0279)$ \\
\hline & $\mathrm{QR}$ & 0.1387 & 0.0974 & 0.0968 & 0.1116 & 0.1314 & 0.1877 & 0.2846 & 0.4244 & 0.6750 \\
\hline & & $(0.0788)$ & $(0.0385)$ & $(0.0391)$ & $(0.0435)$ & $(0.0488)$ & $(0.0465)$ & $(0.0429)$ & $(0.0586)$ & $(0.0984)$ \\
\hline \multirow{6}{*}{$f_{3}$} & FAGL1 & 0.0953 & 0.0994 & 0.1000 & 0.1010 & 0.1046 & 0.1129 & 0.1287 & 0.1482 & 0.1695 \\
\hline & & $(0.0390)$ & $(0.0398)$ & $(0.0398)$ & $(0.0400)$ & $(0.0414)$ & $(0.0433)$ & $(0.0491)$ & $(0.0654)$ & $(0.1006)$ \\
\hline & FAGL2 & 0.1055 & 0.1037 & 0.1030 & 0.1038 & 0.1061 & 0.1087 & 0.1125 & 0.1177 & 0.1220 \\
\hline & & $(0.0396)$ & $(0.0398)$ & $(0.0389)$ & $(0.0391)$ & $(0.0399)$ & $(0.0406)$ & $(0.0428)$ & $(0.0472)$ & $(0.0548)$ \\
\hline & $\mathrm{QR}$ & 0.1390 & 0.1080 & 0.0974 & 0.1122 & 0.1230 & 0.1390 & 0.1784 & 0.2308 & 0.3254 \\
\hline & & $(0.0629)$ & $(0.0534)$ & $(0.0423)$ & $(0.0469)$ & $(0.0488)$ & $(0.0552)$ & $(0.0634)$ & $(0.0748)$ & $(0.1459)$ \\
\hline
\end{tabular}

Table 2.20: True proportion of correctly identifying the model in Example 2.2 when $n=100$ and $F(\cdot)$ is the Student's t distribution function with scale parameter $1 / 3$ and degrees of freedom 3 .

\begin{tabular}{ccccccccc}
\hline$f_{1}$ & $\boldsymbol{d}_{1}^{2}=\mathbf{0}$ & $\boldsymbol{d}_{1}^{3}=\mathbf{0}$ & $\boldsymbol{d}_{1}^{4}=\mathbf{0}$ & $\boldsymbol{d}_{1}^{5}=\mathbf{0}$ & $\boldsymbol{d}_{1}^{6}=\mathbf{0}$ & $\boldsymbol{d}_{1}^{7}=\mathbf{0}$ & $\boldsymbol{d}_{1}^{8}=\mathbf{0}$ & $\boldsymbol{d}_{1}^{9}=\mathbf{0}$ \\
\hline FAGL1 & 0.35 & 0.68 & 0.60 & 0.39 & 0.25 & 0.19 & 0.36 & 0.68 \\
FAGL2 & 0.44 & 0.44 & 0.44 & 0.44 & 0.44 & 0.44 & 0.44 & 0.44 \\
\hline$f_{2}$ & $\boldsymbol{d}_{2}^{2}=\mathbf{0}$ & $\boldsymbol{d}_{2}^{3}=\mathbf{0}$ & $\boldsymbol{d}_{2}^{4}=\mathbf{0}$ & $\boldsymbol{d}_{2}^{5} \neq \mathbf{0}$ & $\boldsymbol{d}_{2}^{6} \neq \mathbf{0}$ & $\boldsymbol{d}_{2}^{7} \neq \mathbf{0}$ & $\boldsymbol{d}_{2}^{8} \neq \mathbf{0}$ & $\boldsymbol{d}_{2}^{9} \neq \mathbf{0}$ \\
\hline FAGL1 & 0.77 & 0.93 & 0.77 & 0.53 & 0.90 & 0.91 & 0.75 & 0.44 \\
FAGL2 & 0.40 & 0.40 & 0.40 & 0.60 & 0.60 & 0.60 & 0.60 & 0.60 \\
\hline$f_{3}$ & $\boldsymbol{d}_{3}^{2}=\mathbf{0}$ & $\boldsymbol{d}_{3}^{3}=\mathbf{0}$ & $\boldsymbol{d}_{3}^{4}=\mathbf{0}$ & $\boldsymbol{d}_{3}^{5}=\mathbf{0}$ & $\boldsymbol{d}_{3}^{6}=\mathbf{0}$ & $\boldsymbol{d}_{3}^{7}=\mathbf{0}$ & $\boldsymbol{d}_{3}^{8}=\mathbf{0}$ & $\boldsymbol{d}_{3}^{9}=\mathbf{0}$ \\
\hline FAGL1 & 0.64 & 0.82 & 0.74 & 0.56 & 0.31 & 0.22 & 0.36 & 0.66 \\
FAGL2 & 0.51 & 0.51 & 0.51 & 0.51 & 0.51 & 0.51 & 0.51 & 0.51 \\
\hline \hline
\end{tabular}


Table 2.21: Summary of $P E$ in Example 2.2 when $n=100$ and $F(\cdot)$ is the Student's t distribution function with scale parameter $1 / 3$ and degrees of freedom 3. Standard errors based on simulations are shown in the parentheses.

\begin{tabular}{cccccccccc}
\hline$\tau$ & 0.1 & 0.2 & 0.3 & 0.4 & 0.5 & 0.6 & 0.7 & 0.8 & 0.9 \\
\hline FAGL1 & 0.1016 & 0.1365 & 0.1701 & 0.1967 & 0.2158 & 0.2263 & 0.2255 & 0.2106 & 0.1620 \\
& $(0.0115)$ & $(0.0118)$ & $(0.0115)$ & $(0.0113)$ & $(0.0114)$ & $(0.0120)$ & $(0.0142)$ & $(0.0495)$ & $(0.0256)$ \\
\hline FAGL2 & 0.0981 & 0.1366 & 0.1710 & 0.1981 & 0.2169 & 0.2260 & 0.2236 & 0.2083 & 0.1617 \\
& $(0.0102)$ & $(0.0110)$ & $(0.0111)$ & $(0.0112)$ & $(0.0114)$ & $(0.0119)$ & $(0.0136)$ & $(0.0416)$ & $(0.0237)$ \\
\hline \multirow{2}{*}{ QR } & 0.0961 & 0.1341 & 0.1683 & 0.1985 & 0.2200 & 0.2317 & 0.2336 & 0.2250 & 0.1866 \\
& $(0.0142)$ & $(0.0121)$ & $(0.0120)$ & $(0.0118)$ & $(0.0125)$ & $(0.0120)$ & $(0.0139)$ & $(0.0688)$ & $(0.0429)$ \\
\hline \hline
\end{tabular}

Table 2.22: Summary of $\sqrt{\widehat{I S E}}$ for non-parametric functions $f_{1}, f_{2}$ and $f_{3}$ in Example 2.2 when $n=200$ and $F(\cdot)$ is the Student's t distribution function with scale parameter $1 / 3$ and degrees of freedom 3. Standard errors based on simulations are shown in the parentheses.

\begin{tabular}{|c|c|c|c|c|c|c|c|c|c|c|}
\hline & $\tau$ & 0.1 & 0.2 & 0.3 & 0.4 & 0.5 & 0.6 & 0.7 & 0.8 & 0.9 \\
\hline \multirow{3}{*}{$f_{1}$} & FAGL1 & $\begin{array}{c}0.0724 \\
(0.0181)\end{array}$ & $\begin{array}{c}0.0646 \\
(0.0206)\end{array}$ & $\begin{array}{c}0.0642 \\
(0.0209)\end{array}$ & $\begin{array}{c}0.0647 \\
(0.0214)\end{array}$ & $\begin{array}{c}0.0681 \\
(0.0236)\end{array}$ & $\begin{array}{c}0.0739 \\
(0.0271)\end{array}$ & $\begin{array}{c}0.0838 \\
(0.0354)\end{array}$ & $\begin{array}{c}0.0990 \\
(0.0470)\end{array}$ & $\begin{array}{c}0.1167 \\
(0.0588)\end{array}$ \\
\hline & FAGL2 & $\begin{array}{c}0.0707 \\
(0.0241)\end{array}$ & $\begin{array}{c}0.0660 \\
(0.0218)\end{array}$ & $\begin{array}{c}0.0654 \\
(0.0219)\end{array}$ & $\begin{array}{c}0.0669 \\
(0.0230)\end{array}$ & $\begin{array}{c}0.0694 \\
(0.0246)\end{array}$ & $\begin{array}{c}0.0722 \\
(0.0270)\end{array}$ & $\begin{array}{c}0.0761 \\
(0.0312)\end{array}$ & $\begin{array}{c}0.0806 \\
(0.0362)\end{array}$ & $\begin{array}{c}0.0856 \\
(0.0387)\end{array}$ \\
\hline & QR & $\begin{array}{c}0.0886 \\
(0.0324) \\
\end{array}$ & $\begin{array}{c}0.0596 \\
(0.0200) \\
\end{array}$ & $\begin{array}{c}0.0549 \\
(0.0196) \\
\end{array}$ & $\begin{array}{c}0.0590 \\
(0.0198) \\
\end{array}$ & $\begin{array}{c}0.0781 \\
(0.0271) \\
\end{array}$ & $\begin{array}{c}0.0922 \\
(0.0323) \\
\end{array}$ & $\begin{array}{c}0.1145 \\
(0.0459) \\
\end{array}$ & $\begin{array}{c}0.1437 \\
(0.0705) \\
\end{array}$ & $\begin{array}{c}0.2293 \\
(0.1030) \\
\end{array}$ \\
\hline \multirow{3}{*}{$f_{2}$} & FAGL1 & $\begin{array}{c}0.0735 \\
(0.0273)\end{array}$ & $\begin{array}{c}0.0747 \\
(0.0271)\end{array}$ & $\begin{array}{c}0.0748 \\
(0.0271)\end{array}$ & $\begin{array}{c}0.0764 \\
(0.0282)\end{array}$ & $\begin{array}{c}0.0836 \\
(0.0334)\end{array}$ & $\begin{array}{c}0.1368 \\
(0.0234)\end{array}$ & $\begin{array}{c}0.2336 \\
(0.0196)\end{array}$ & $\begin{array}{c}0.3692 \\
(0.0245)\end{array}$ & $\begin{array}{c}0.6053 \\
(0.0262)\end{array}$ \\
\hline & FAGL2 & $\begin{array}{c}0.0912 \\
(0.0329)\end{array}$ & $\begin{array}{c}0.0813 \\
(0.0291)\end{array}$ & $\begin{array}{c}0.0805 \\
(0.0294)\end{array}$ & $\begin{array}{c}0.0848 \\
(0.0312)\end{array}$ & $\begin{array}{c}0.0907 \\
(0.0337)\end{array}$ & $\begin{array}{c}0.1336 \\
(0.0209)\end{array}$ & $\begin{array}{c}0.2287 \\
(0.0156)\end{array}$ & $\begin{array}{c}0.3667 \\
(0.0167)\end{array}$ & $\begin{array}{c}0.6129 \\
(0.0213)\end{array}$ \\
\hline & $\mathrm{QR}$ & $\begin{array}{c}0.0889 \\
(0.0288) \\
\end{array}$ & $\begin{array}{c}0.0667 \\
(0.0221) \\
\end{array}$ & $\begin{array}{c}0.0587 \\
(0.0225) \\
\end{array}$ & $\begin{array}{c}0.0665 \\
(0.0273) \\
\end{array}$ & $\begin{array}{c}0.0905 \\
(0.0395) \\
\end{array}$ & $\begin{array}{c}0.1530 \\
(0.0314) \\
\end{array}$ & $\begin{array}{c}0.2502 \\
(0.0285) \\
\end{array}$ & $\begin{array}{c}0.3876 \\
(0.0428) \\
\end{array}$ & $\begin{array}{c}0.6306 \\
(0.0728) \\
\end{array}$ \\
\hline \multirow{3}{*}{$f_{3}$} & FAGL1 & $\begin{array}{c}0.0607 \\
(0.0224)\end{array}$ & $\begin{array}{c}0.0623 \\
(0.0224)\end{array}$ & $\begin{array}{c}0.0628 \\
(0.0225)\end{array}$ & $\begin{array}{c}0.0638 \\
(0.0229)\end{array}$ & $\begin{array}{c}0.0669 \\
(0.0233)\end{array}$ & $\begin{array}{c}0.0745 \\
(0.0256)\end{array}$ & $\begin{array}{c}0.0859 \\
(0.0326)\end{array}$ & $\begin{array}{c}0.1018 \\
(0.0435)\end{array}$ & $\begin{array}{c}0.1248 \\
(0.0685)\end{array}$ \\
\hline & FAGL2 & $\begin{array}{c}0.0683 \\
(0.0243)\end{array}$ & $\begin{array}{c}0.0668 \\
(0.0234)\end{array}$ & $\begin{array}{c}0.0665 \\
(0.0230)\end{array}$ & $\begin{array}{c}0.0675 \\
(0.0233)\end{array}$ & $\begin{array}{c}0.0694 \\
(0.0240)\end{array}$ & $\begin{array}{c}0.0718 \\
(0.0254)\end{array}$ & $\begin{array}{c}0.0743 \\
(0.0276)\end{array}$ & $\begin{array}{c}0.0779 \\
(0.0318)\end{array}$ & $\begin{array}{c}0.0827 \\
(0.0383)\end{array}$ \\
\hline & $\mathrm{QR}$ & $\begin{array}{c}0.0863 \\
(0.0299)\end{array}$ & $\begin{array}{c}0.0601 \\
(0.0207)\end{array}$ & $\begin{array}{c}0.0557 \\
(0.0201)\end{array}$ & $\begin{array}{c}0.0621 \\
(0.0241)\end{array}$ & $\begin{array}{c}0.0750 \\
(0.0233)\end{array}$ & $\begin{array}{c}0.0942 \\
(0.0268)\end{array}$ & $\begin{array}{c}0.1203 \\
(0.0356)\end{array}$ & $\begin{array}{c}0.1516 \\
(0.0623)\end{array}$ & $\begin{array}{c}0.2486 \\
(0.1237)\end{array}$ \\
\hline
\end{tabular}


Table 2.23: True proportion of correctly identifying the model in Example 2.2 when $n=200$ and $F(\cdot)$ is the Student's t distribution function with scale parameter $1 / 3$ and degrees of freedom 3 .

\begin{tabular}{ccccccccc}
\hline$f_{1}$ & $\boldsymbol{d}_{1}^{2}=\mathbf{0}$ & $\boldsymbol{d}_{1}^{3}=\mathbf{0}$ & $\boldsymbol{d}_{1}^{4}=\mathbf{0}$ & $\boldsymbol{d}_{1}^{5}=\mathbf{0}$ & $\boldsymbol{d}_{1}^{6}=\mathbf{0}$ & $\boldsymbol{d}_{1}^{7}=\mathbf{0}$ & $\boldsymbol{d}_{1}^{8}=\mathbf{0}$ & $\boldsymbol{d}_{1}^{9}=\mathbf{0}$ \\
\hline FAGL1 & 0.15 & 0.68 & 0.57 & 0.36 & 0.22 & 0.22 & 0.24 & 0.55 \\
FAGL2 & 0.25 & 0.25 & 0.25 & 0.25 & 0.25 & 0.25 & 0.25 & 0.25 \\
\hline$f_{2}$ & $\boldsymbol{d}_{2}^{2}=\mathbf{0}$ & $\boldsymbol{d}_{2}^{3}=\mathbf{0}$ & $\boldsymbol{d}_{2}^{4}=\mathbf{0}$ & $\boldsymbol{d}_{2}^{5} \neq \mathbf{0}$ & $\boldsymbol{d}_{2}^{6} \neq \mathbf{0}$ & $\boldsymbol{d}_{2}^{7} \neq \mathbf{0}$ & $\boldsymbol{d}_{2}^{8} \neq \mathbf{0}$ & $\boldsymbol{d}_{2}^{9} \neq \mathbf{0}$ \\
\hline FAGL1 & 0.67 & 0.92 & 0.79 & 0.80 & 0.96 & 0.96 & 0.90 & 0.51 \\
FAGL2 & 0.08 & 0.08 & 0.08 & 0.92 & 0.92 & 0.92 & 0.92 & 0.92 \\
\hline$f_{3}$ & $\boldsymbol{d}_{3}^{2}=\mathbf{0}$ & $\boldsymbol{d}_{3}^{3}=\mathbf{0}$ & $\boldsymbol{d}_{3}^{4}=\mathbf{0}$ & $\boldsymbol{d}_{3}^{5}=\mathbf{0}$ & $\boldsymbol{d}_{3}^{6}=\mathbf{0}$ & $\boldsymbol{d}_{3}^{7}=\mathbf{0}$ & $\boldsymbol{d}_{3}^{8}=\mathbf{0}$ & $\boldsymbol{d}_{3}^{9}=\mathbf{0}$ \\
\hline FAGL1 & 0.58 & 0.74 & 0.68 & 0.43 & 0.20 & 0.20 & 0.32 & 0.47 \\
FAGL2 & 0.45 & 0.45 & 0.45 & 0.45 & 0.45 & 0.45 & 0.45 & 0.45 \\
\hline \hline
\end{tabular}

Table 2.24: Summary of $P E$ in Example 2.2 when $n=200$ and $F(\cdot)$ is the Student's t distribution function with scale parameter $1 / 3$ and degrees of freedom 3. Standard errors based on simulations are shown in the parentheses.

\begin{tabular}{cccccccccc}
\hline$\tau$ & 0.1 & 0.2 & 0.3 & 0.4 & 0.5 & 0.6 & 0.7 & 0.8 & 0.9 \\
\hline FAGL1 & 0.0970 & 0.1269 & 0.1567 & 0.1831 & 0.2045 & 0.2175 & 0.2182 & 0.2034 & 0.1641 \\
& $(0.0104)$ & $(0.0081)$ & $(0.0062)$ & $(0.0061)$ & $(0.0073)$ & $(0.0087)$ & $(0.0105)$ & $(0.0120)$ & $(0.0130)$ \\
\hline FAGL2 & 0.0937 & 0.1258 & 0.1569 & 0.1847 & 0.2063 & 0.2187 & 0.2192 & 0.2051 & 0.1672 \\
& $(0.0087)$ & $(0.0069)$ & $(0.0060)$ & $(0.0071)$ & $(0.0084)$ & $(0.0096)$ & $(0.0109)$ & $(0.0118)$ & $(0.0126)$ \\
\hline QR & 0.0788 & 0.1199 & 0.1536 & 0.1815 & 0.2042 & 0.2161 & 0.2155 & 0.1971 & 0.1551 \\
& $(0.0040)$ & $(0.0048)$ & $(0.0055)$ & $(0.0057)$ & $(0.0059)$ & $(0.0055)$ & $(0.0064)$ & $(0.0089)$ & $(0.0103)$ \\
\hline \hline
\end{tabular}


meteorological variables, collected by the Norwegian Public Roads Administration. It is available online at http://lib.stat.cmu.edu/datasets/ N02.dat. There are 500 observations, 6 predictors and one response variable. The response variable is the hourly values of the logarithm of the concentration of $\mathrm{NO}_{2}$ particles $(\log \mathrm{NO} 2)$, measured at Alnabru in Oslo, Norway, between October 2001 and August 2003. The 6 predictors are the logarithm of the number of cars per hour (logNoCars), temperature 2 meters above ground (TEMP2), wind speed, the temperature difference between 25 meters and 2 meters above ground (TEMPDIFF252), wind direction, and hour of day.

We apply the two proposed penalized methods, as well as standard additive quantile regression, to investigate the effects of the 6 predictors on 9 different quantiles $\{0.1,0.2, \ldots, 0.9\}$. We also compare the proposed methods with the methods proposed by Jiang et al. (2013), which used fused adaptive LASSO (FAL) and fused adaptive sup-norm (FAS) penalties in the linear quantile regression model. We perform a ten-fold cross-validation (Picard and Cook (1984)) to calculate the prediction error (PE) as defined for our simulation studies. We have $P E=1.917(s d=0.142)$ for FAGL1, $P E=1.891(s d=0.152)$ for FAGL2, $P E=1.920(s d=0.195)$ for $\mathrm{QR}$, $P E=1.930(s d=0.213)$ for FAL, and $P E=1.932(s d=0.215)$ for FAS. These results indicate that FAGL2 yields better prediction accuracy than other methods. Table 2.25 shows the proportion of times (out of 10 times) each interquantile difference is identified to be 0 at adjacent quantile levels. We also show the estimated functions obtained from FAGL1 and FAGL2, in Figure 2.1 and Figure 2.2, respectively. 
Table 2.25: $\mathrm{NO}_{2}$ particles data. The proportion of times the interquantile differences are shrunk to zero.

\begin{tabular}{ccccccccc}
\hline logNoCars & $\boldsymbol{d}_{1}^{2}=\mathbf{0}$ & $\boldsymbol{d}_{1}^{3}=\mathbf{0}$ & $\boldsymbol{d}_{1}^{4}=\mathbf{0}$ & $\boldsymbol{d}_{1}^{5}=\mathbf{0}$ & $\boldsymbol{d}_{1}^{6}=\mathbf{0}$ & $\boldsymbol{d}_{1}^{7}=\mathbf{0}$ & $\boldsymbol{d}_{1}^{8}=\mathbf{0}$ & $\boldsymbol{d}_{1}^{9}=\mathbf{0}$ \\
\hline FAGL1 & 0.3 & 0 & 0 & 0.1 & 0.4 & 0.8 & 1 & 1 \\
FAGL2 & 0.2 & 0.2 & 0.2 & 0.2 & 0.2 & 0.2 & 0.2 & 0.2 \\
\hline TEMP2 & $\boldsymbol{d}_{2}^{2}=\mathbf{0}$ & $\boldsymbol{d}_{2}^{3}=\mathbf{0}$ & $\boldsymbol{d}_{2}^{4}=\mathbf{0}$ & $\boldsymbol{d}_{2}^{5}=\mathbf{0}$ & $\boldsymbol{d}_{2}^{6}=\mathbf{0}$ & $\boldsymbol{d}_{2}^{7}=\mathbf{0}$ & $\boldsymbol{d}_{2}^{8}=\mathbf{0}$ & $\boldsymbol{d}_{2}^{9}=\mathbf{0}$ \\
\hline FAGL1 & 0.2 & 1 & 0.9 & 0.1 & 0 & 0 & 0.8 & 1 \\
FAGL2 & 0 & 0 & 0 & 0 & 0 & 0 & 0 & 0 \\
\hline Wind Speed & $\boldsymbol{d}_{3}^{2}=\mathbf{0}$ & $\boldsymbol{d}_{3}^{3}=\mathbf{0}$ & $\boldsymbol{d}_{3}^{4}=\mathbf{0}$ & $\boldsymbol{d}_{3}^{5}=\mathbf{0}$ & $\boldsymbol{d}_{3}^{6}=\mathbf{0}$ & $\boldsymbol{d}_{3}^{7}=\mathbf{0}$ & $\boldsymbol{d}_{3}^{8}=\mathbf{0}$ & $\boldsymbol{d}_{3}^{9}=\mathbf{0}$ \\
\hline FAGL1 & 0.3 & 0.7 & 0.2 & 0.1 & 0.7 & 0.7 & 1 & 1 \\
FAGL2 & 0.2 & 0.2 & 0.2 & 0.2 & 0.2 & 0.2 & 0.2 & 0.2 \\
\hline TEMPDIFF252 & $\boldsymbol{d}_{4}^{2}=\mathbf{0}$ & $\boldsymbol{d}_{4}^{3}=\mathbf{0}$ & $\boldsymbol{d}_{4}^{4}=\mathbf{0}$ & $\boldsymbol{d}_{4}^{5}=\mathbf{0}$ & $\boldsymbol{d}_{4}^{6}=\mathbf{0}$ & $\boldsymbol{d}_{4}^{7}=\mathbf{0}$ & $\boldsymbol{d}_{4}^{8}=\mathbf{0}$ & $\boldsymbol{d}_{4}^{9}=\mathbf{0}$ \\
\hline FAGL1 & 0.5 & 1 & 1 & 0 & 0 & 0 & 0.4 & 1 \\
FAGL2 & 0.5 & 0.5 & 0.5 & 0.5 & 0.5 & 0.5 & 0.5 & 0.5 \\
\hline Wind Direction & $\boldsymbol{d}_{5}^{2}=\mathbf{0}$ & $\boldsymbol{d}_{5}^{3}=\mathbf{0}$ & $\boldsymbol{d}_{5}^{4}=\mathbf{0}$ & $\boldsymbol{d}_{5}^{5}=\mathbf{0}$ & $\boldsymbol{d}_{5}^{6}=\mathbf{0}$ & $\boldsymbol{d}_{5}^{7}=\mathbf{0}$ & $\boldsymbol{d}_{5}^{8}=\mathbf{0}$ & $\boldsymbol{d}_{5}^{9}=\mathbf{0}$ \\
\hline FAGL1 & 0 & 0.7 & 0 & 0 & 0 & 0.8 & 1 & 1 \\
FAGL2 & 0 & 0 & 0 & 0 & 0 & 0 & 0 & 0 \\
\hline Hour of Day & $\boldsymbol{d}_{6}^{2}=\mathbf{0}$ & $\boldsymbol{d}_{6}^{3}=\mathbf{0}$ & $\boldsymbol{d}_{6}^{4}=\mathbf{0}$ & $\boldsymbol{d}_{6}^{5}=\mathbf{0}$ & $\boldsymbol{d}_{6}^{6}=\mathbf{0}$ & $\boldsymbol{d}_{6}^{7}=\mathbf{0}$ & $\boldsymbol{d}_{6}^{8}=\mathbf{0}$ & $\boldsymbol{d}_{6}^{9}=\mathbf{0}$ \\
\hline FAGL1 & 0.7 & 0.5 & 0 & 0.1 & 0.8 & 1 & 1 & 1 \\
FAGL2 & 0.6 & 0.6 & 0.6 & 0.6 & 0.6 & 0.6 & 0.6 & 0.6 \\
\hline \hline
\end{tabular}



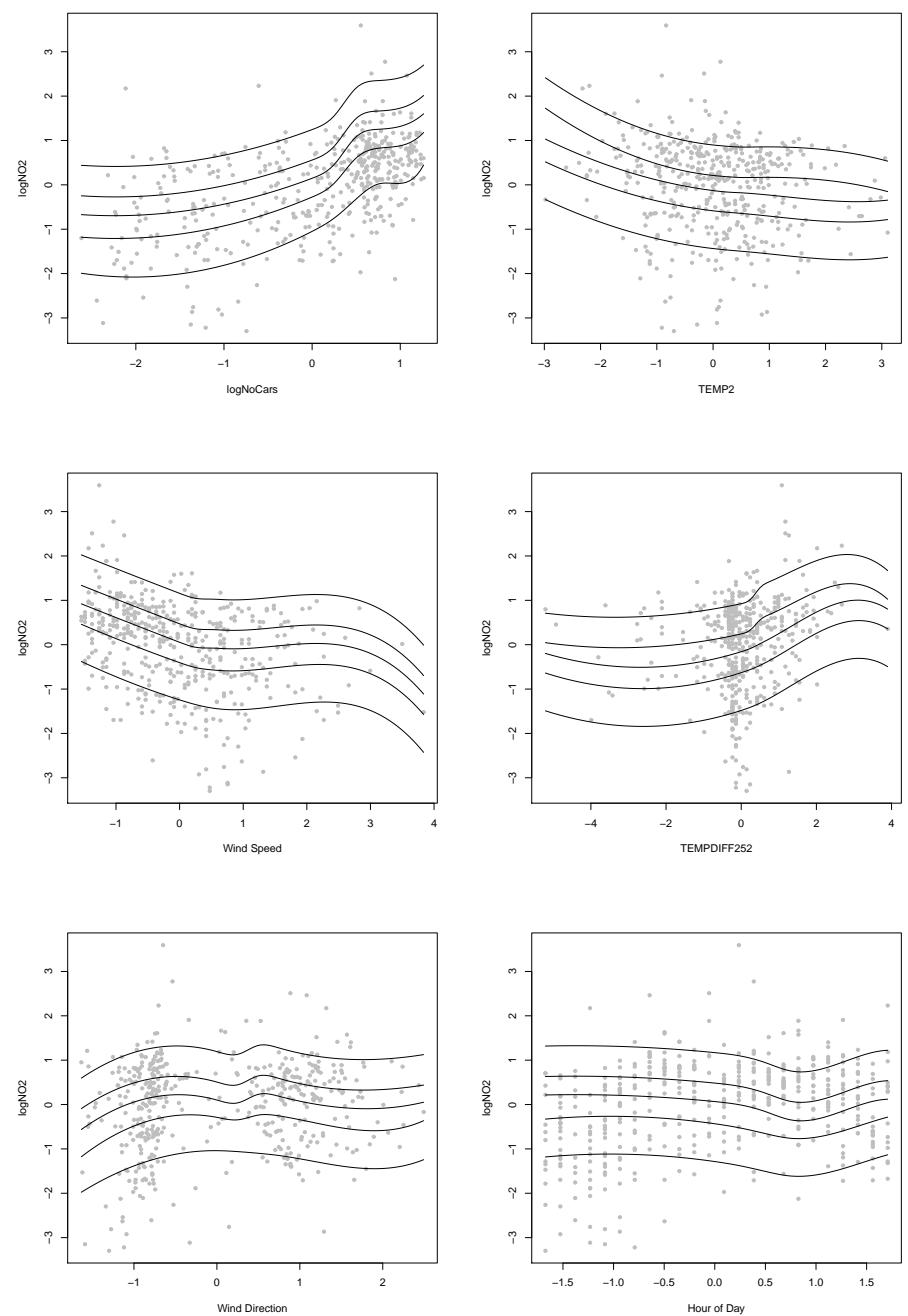

Figure 2.1: $\mathrm{NO}_{2}$ particles data. Estimated functions with one predictor varying and the others fixed at 0 . The solid black lines are the estimated conditional quantiles by FAGL1 at five different quantile levels 0.1, 0.3, 0.5, 0.7 and 0.9 . 

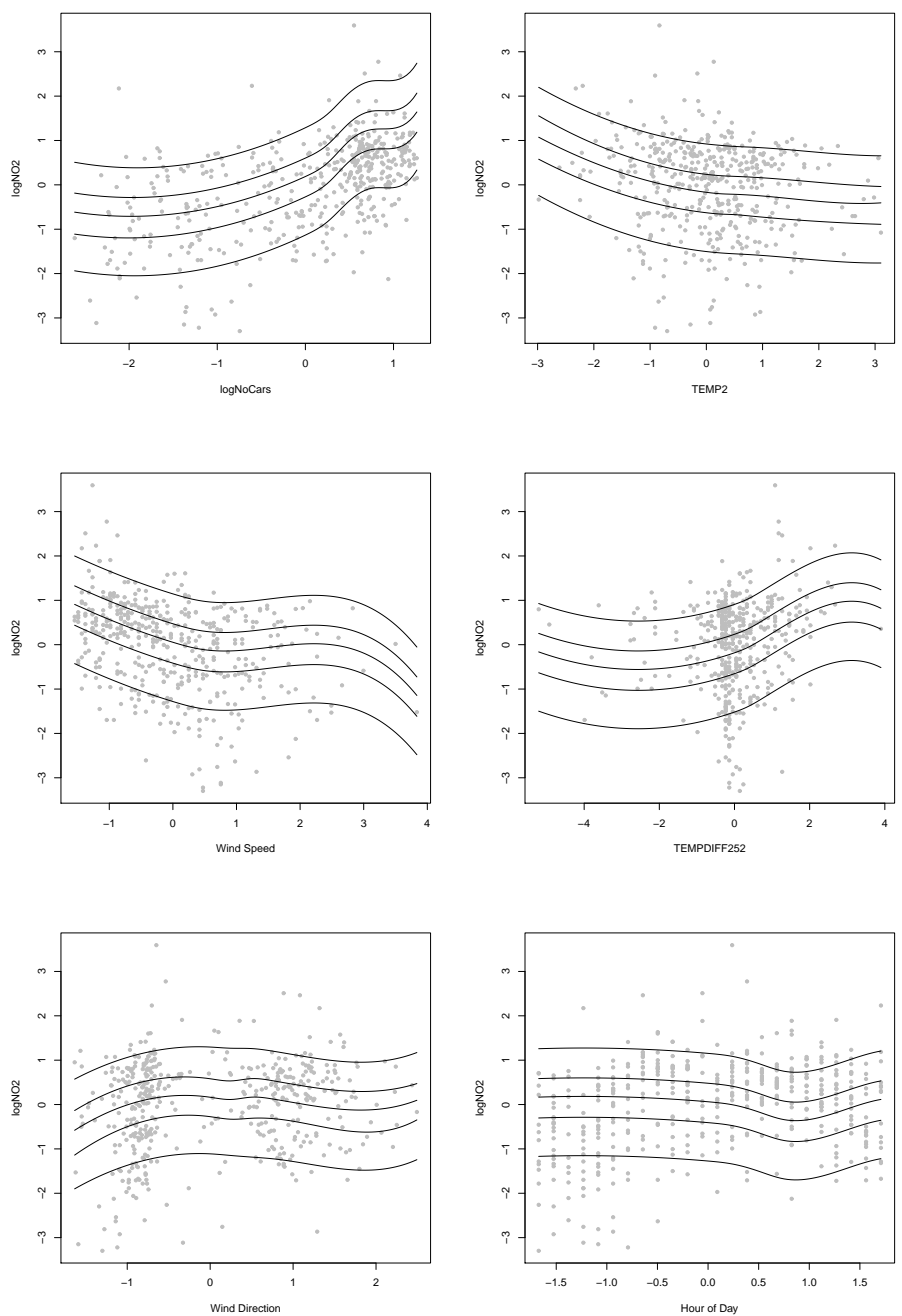

Figure 2.2: $\mathrm{NO}_{2}$ particles data. Estimated functions with one predictor varying and the others fixed at 0 . The solid black lines are the estimated conditional quantiles by FAGL2 at five different quantile levels $0.1,0.3,0.5$, 0.7 and 0.9 . 


\subsection{Summary}

In this chapter, we propose two fused adaptive group LASSO penalties to induce interquantile shrinkage in additive regression models. The two proposed penalized methods are able to estimate the quantile functions and identify quantile regions where non-parametric functions vary. Simulation studies and a real data application show the proposed methods have favourable performances compared to standard additive quantile regression.

\subsection{Appendix}

We first present two lemmas which are important for the subsequent proof of the theorems.

Lemma 2.1. Assume condition (A3) holds. Then, we have

$$
\max _{1 \leq i \leq n, 1 \leq k \leq K}\left\|\mathbf{z}_{i k}\right\|=O_{p}(1) .
$$

Proof. From the definition of $\mathbf{z}_{i k}$, we have

$$
\begin{aligned}
\left\|\mathbf{z}_{i k}\right\|^{2} & =\mathbf{z}_{i k}^{T} \mathbf{z}_{i k} \\
& =\left(1, \boldsymbol{\Pi}\left(\mathbf{X}_{i}\right)^{T}\right) \mathbf{T}_{k} \mathbf{T}_{k}^{T}\left(1, \boldsymbol{\Pi}\left(\mathbf{X}_{i}\right)^{T}\right)^{T} \\
& =1+k\left\|\boldsymbol{\Pi}\left(\mathbf{X}_{i}\right)\right\|^{2} \\
& =O_{p}(1),
\end{aligned}
$$

since

$$
\mathbf{T}_{k} \mathbf{T}_{k}^{T}=\left(\begin{array}{ccccc}
1 & 0 & 0 & \cdots & 0 \\
0 & k & 0 & \cdots & 0 \\
0 & 0 & k & \cdots & 0 \\
\cdots & \cdots & \cdots & \cdots & \cdots \\
0 & 0 & 0 & \cdots & k
\end{array}\right)_{\left(1+p m_{n}\right) \times\left(1+p m_{n}\right)}
$$


The following lemma was shown in Wang et al. (2009). To match the normalization used in Wang et al. (2009), we define $\mathbf{Z}_{i k}^{T}=\sqrt{\frac{k_{n}}{n}} \mathbf{z}_{i k}^{T}, \mu_{k}^{*}=$ $\sqrt{\frac{n}{k_{n}}} \mu_{k}, \mu_{0 k}^{*}=\sqrt{\frac{n}{k_{n}}} \mu_{0 k}, \boldsymbol{\beta}^{k *}=\sqrt{\frac{n}{k_{n}}} \boldsymbol{\beta}^{k}, \boldsymbol{\beta}_{0}^{k *}=\sqrt{\frac{n}{k_{n}}} \boldsymbol{\beta}_{0}^{k}, \boldsymbol{\theta}^{*}=\sqrt{\frac{n}{k_{n}}} \boldsymbol{\theta}$ and $\boldsymbol{\theta}_{0}^{*}=\sqrt{\frac{n}{k_{n}}} \boldsymbol{\theta}_{0}$.

Lemma 2.2. (Wang et al., 2009) Under the conditions $(A 1)-(A 3)$, for $k=1, \ldots, K$, we have,

(i) for any sequence $\left\{L_{n}\right\}$ satisfying $0 \leq L_{n} \leq k_{n}^{\delta_{0} / 10}$ for some $0<\delta_{0}<$ $(d-1 / 2) /(2 d+1)$,

$$
\begin{array}{r}
\sup _{\|\boldsymbol{\theta}\| \leq L_{n} k_{n}^{1 / 2}} k_{n}^{-1} \mid \sum_{i=1}^{n}\left[\rho_{\tau_{k}}\left(\epsilon_{i k}-\mathbf{Z}_{i k}^{T} \boldsymbol{\theta}-R^{k}\left(\mathbf{X}_{i}\right)\right)-\rho_{\tau_{k}}\left(\epsilon_{i k}-R^{k}\left(\mathbf{X}_{i}\right)\right)\right. \\
+\mathbf{Z}_{i k}^{T} \boldsymbol{\theta}\left(\tau_{k}-I\left(\epsilon_{i k} \leq 0\right)\right)-\mathrm{E}\left(\rho_{\tau_{k}}\left(\epsilon_{i k}-\mathbf{Z}_{i k}^{T} \boldsymbol{\theta}-R^{k}\left(\mathbf{X}_{i}\right)\right)\right. \\
\left.\left.-\rho_{\tau_{k}}\left(\epsilon_{i k}-R^{k}\left(\mathbf{X}_{i}\right)\right)\right)\right] \mid=o_{p}(1)
\end{array}
$$

(ii) for any $\varepsilon>0$, there exists $L$ (sufficient large) such that as $n \rightarrow \infty$,

$$
\begin{aligned}
\mathrm{P}\left\{k _ { n } ^ { - 1 } \left(\inf _{\|\boldsymbol{\theta}\|=L k_{n}^{1 / 2}} \sum_{i=1}^{n}[\right.\right. & {\left[\mathrm{E}\left(\rho_{\tau_{k}}\left(\epsilon_{i k}-\mathbf{Z}_{i k}^{T} \boldsymbol{\theta}-R^{k}\left(\mathbf{X}_{i}\right)\right)-\rho_{\tau_{k}}\left(\epsilon_{i k}-R^{k}\left(\mathbf{X}_{i}\right)\right)\right)\right] } \\
& \left.\left.-L k_{n}^{1 / 2}\left\|\sum_{i=1}^{n} \mathbf{Z}_{i k}\left(\tau_{k}-I\left(\epsilon_{i k} \leq 0\right)\right)\right\|\right)>1\right\}>1-\varepsilon .
\end{aligned}
$$

\section{Proof of Theorem 2.2}

Let

$$
\begin{array}{r}
Q_{1}^{*}\left(\boldsymbol{\theta}^{*}\right)=\sum_{k=1}^{K} \sum_{i=1}^{n} \rho_{\tau_{k}}\left(\epsilon_{i k}-R^{k}\left(\mathbf{X}_{i}\right)-\mathbf{Z}_{i k}^{T}\left(\boldsymbol{\theta}^{*}-\boldsymbol{\theta}_{0}^{*}\right)\right) \\
+\lambda_{n} \sum_{k=2}^{K} \sum_{j=1}^{p} \sqrt{\frac{k_{n}}{n}} \omega_{j, k}\left\|\mathbf{d}_{j}^{k *}\right\|,
\end{array}
$$


and

$$
\hat{\boldsymbol{\theta}}^{*}=\sqrt{\frac{n}{k_{n}}} \hat{\boldsymbol{\theta}}=\underset{\boldsymbol{\theta}^{*} \in \mathbb{R}^{K+K p m_{n}}}{\arg \min } Q_{1}^{*}\left(\boldsymbol{\theta}^{*}\right) .
$$

We have

$$
\begin{aligned}
& \frac{1}{n} \sum_{i=1}^{n}\left(\hat{f}_{j}^{k}\left(X_{i j}\right)-f_{0 j}^{k}\left(X_{i j}\right)\right)^{2} \\
= & \frac{1}{n} \sum_{i=1}^{n}\left(\boldsymbol{\Pi}_{j}\left(X_{i j}\right)^{T} \hat{\boldsymbol{\beta}}_{j}^{k}-\boldsymbol{\Pi}_{j}\left(X_{i j}\right)^{T} \boldsymbol{\beta}_{0 j}^{k}+r_{j}^{k}\left(X_{i j}\right)\right)^{2} \\
\leq & \frac{2}{n} \sum_{i=1}^{n}\left(\boldsymbol{\Pi}_{j}\left(X_{i j}\right)^{T}\left(\hat{\boldsymbol{\beta}}_{j}^{k}-\boldsymbol{\beta}_{0 j}^{k}\right)\right)^{2}+O\left(k_{n}^{-2 d}\right) \\
= & O_{p}\left(\frac{1}{k_{n}}\right)\left\|\hat{\boldsymbol{\beta}}_{j}^{k}-\boldsymbol{\beta}_{0 j}^{k}\right\|^{2}+O\left(k_{n}^{-2 d}\right) .
\end{aligned}
$$

In order to get the convergence rate, it suffices to show that $\left\|\hat{\boldsymbol{\beta}}^{k}-\boldsymbol{\beta}_{0}^{k}\right\|^{2}=$ $O_{p}\left(k_{n}^{2} / n\right)$. From $\boldsymbol{\beta}^{k}=\sqrt{\frac{k_{n}}{n}} \boldsymbol{\beta}^{k *}$, we need to show $\left\|\hat{\boldsymbol{\beta}}^{k *}-\boldsymbol{\beta}_{0}^{k *}\right\|^{2}=O_{p}\left(k_{n}\right)$. Since

$$
\mathbf{T}_{k} \boldsymbol{\theta}^{*}=\left(\begin{array}{c}
\mu_{k}^{*} \\
\boldsymbol{\beta}^{k *}
\end{array}\right)
$$

it suffices to show that $\left\|\hat{\boldsymbol{\theta}}^{*}-\boldsymbol{\theta}_{0}^{*}\right\|^{2}=O_{p}\left(k_{n}\right)$. As in Fan and Li $(2001)$, we only need to show that for any $\varepsilon>0$, there is a sufficiently large constant $C$, such that

$$
\liminf _{n} \mathrm{P}\left\{\inf _{\mathbf{u} \in \mathbb{R}^{K+K p m_{n}}:\|\mathbf{u}\|=C} Q_{1}^{*}\left(\boldsymbol{\theta}_{0}^{*}+k_{n}^{1 / 2} \mathbf{u}\right)>Q_{1}^{*}\left(\boldsymbol{\theta}_{0}^{*}\right)\right\}>1-\varepsilon .
$$

We have

$$
\begin{aligned}
& \inf _{\|\mathbf{u}\|=C} Q_{1}^{*}\left(\boldsymbol{\theta}_{0}^{*}+k_{n}^{1 / 2} \mathbf{u}\right)-Q_{1}^{*}\left(\boldsymbol{\theta}_{0}^{*}\right) \\
= & \inf _{\|\mathbf{u}\|=C} \sum_{k=1}^{K} \sum_{i=1}^{n}\left\{\rho_{\tau_{k}}\left(\epsilon_{i k}-R^{k}\left(\mathbf{X}_{i}\right)-\mathbf{Z}_{i k}^{T} k_{n}^{1 / 2} \mathbf{u}\right)-\rho_{\tau_{k}}\left(\epsilon_{i k}-R^{k}\left(\mathbf{X}_{i}\right)\right)\right\}
\end{aligned}
$$




$$
\begin{aligned}
&+\lambda_{n} \sum_{k=2}^{K} \sum_{j=1}^{p} \sqrt{\frac{k_{n}}{n}} \omega_{j, k}\left[\left\|\mathbf{d}_{0 j}^{k *}+k_{n}^{1 / 2} \mathbf{u}_{(j, k)}\right\|-\left\|\mathbf{d}_{0 j}^{k *}\right\|\right] \\
&= \inf _{\|\mathbf{u}\|=C} \sum_{k=1}^{K} \sum_{i=1}^{n}\left\{\rho_{\tau_{k}}\left(\epsilon_{i k}-R^{k}\left(\mathbf{X}_{i}\right)-\mathbf{Z}_{i k}^{T} k_{n}^{1 / 2} \mathbf{u}\right)-\rho_{\tau_{k}}\left(\epsilon_{i k}-R^{k}\left(\mathbf{X}_{i}\right)\right)\right\} \\
&+\lambda_{n} \sum_{k=2}^{k_{0}} \sum_{j=1}^{p} \sqrt{\frac{k_{n}}{n}} \omega_{j, k}\left[\left\|\mathbf{d}_{0 j}^{k *}+k_{n}^{1 / 2} \mathbf{u}_{(j, k)}\right\|-\left\|\mathbf{d}_{0 j}^{k *}\right\|\right] \\
&+\lambda_{n} \sum_{k=k_{0}+1}^{K} \sum_{j=1}^{p} \sqrt{\frac{k_{n}}{n}} \omega_{j, k}\left\|k_{n}^{1 / 2} \mathbf{u}_{(j, k)}\right\| \\
& \geq \quad \inf _{\|\mathbf{u}\|=C} \sum_{k=1}^{K} \sum_{i=1}^{n}\left\{\rho_{\tau_{k}}\left(\epsilon_{i k}-R^{k}\left(\mathbf{X}_{i}\right)-\mathbf{Z}_{i k}^{T} k_{n}^{1 / 2} \mathbf{u}\right)-\rho_{\tau_{k}}\left(\epsilon_{i k}-R^{k}\left(\mathbf{X}_{i}\right)\right)\right\} \\
& \quad-\lambda_{n} \sum_{k=2}^{k_{0}} \sum_{j=1}^{p} \sqrt{\frac{k_{n}}{n}} \omega_{j, k}\left\|k_{n}^{1 / 2} \mathbf{u}_{(j, k)}\right\|,
\end{aligned}
$$

where $\mathbf{u}_{(j, k)} \in \mathbb{R}^{m_{n}}$ is a vector with the entries taken from $\mathbf{u}$ and the $i$-th entry in $\mathbf{u}_{(j, k)}$ is the $\left(K+(k-1) p m_{n}+(j-1) m_{n}+i\right)$-th entry of $\mathbf{u}$.

For the first term in (2.6), from Lemma 2.2, we have with probability approaching 1,

$$
\begin{aligned}
& \inf _{\|\boldsymbol{\theta}\|=C k_{n}^{1 / 2}} k_{n}^{-1} \sum_{i=1}^{n}\left[\rho_{\tau_{k}}\left(\epsilon_{i k}-\mathbf{Z}_{i k}^{T} \boldsymbol{\theta}-R^{k}\left(\mathbf{X}_{i}\right)\right)-\rho_{\tau_{k}}\left(\epsilon_{i k}-R^{k}\left(\mathbf{X}_{i}\right)\right)\right] \\
\geq & \inf _{\|\boldsymbol{\theta}\|=C k_{n}^{1 / 2}}\left\{k_{n}^{-1} \sum_{i=1}^{n}\left[\mathrm{E}\left(\rho_{\tau_{k}}\left(\epsilon_{i k}-\mathbf{Z}_{i k}^{T} \boldsymbol{\theta}-R^{k}\left(\mathbf{X}_{i}\right)\right)-\rho_{\tau_{k}}\left(\epsilon_{i k}-R^{k}\left(\mathbf{X}_{i}\right)\right)\right)\right]\right. \\
& \left.-C k_{n}^{-1 / 2}\left\|\sum_{i=1}^{n} \mathbf{Z}_{i k}\left(\tau_{k}-I\left(\epsilon_{i k} \leq 0\right)\right)\right\|\right\}-\epsilon \\
> & 1-\epsilon,
\end{aligned}
$$

where $C$ is a constant big enough. Setting $\epsilon=\frac{1}{2}$, we have

$$
\inf _{\|\mathbf{u}\|=C} \sum_{k=1}^{K} \sum_{i=1}^{n}\left\{\rho_{\tau_{k}}\left(\epsilon_{i k}-R^{k}\left(\mathbf{X}_{i}\right)-\mathbf{Z}_{i k}^{T} k_{n}^{1 / 2} \mathbf{u}\right)-\rho_{\tau_{k}}\left(\epsilon_{i k}-R^{k}\left(\mathbf{X}_{i}\right)\right)\right\}>\frac{k_{n}}{2}
$$

where $k_{n} \sim n^{\frac{1}{2 d+1}}$. 
Next, we consider the second term in (2.6). Under the model assumption that each true non-parametric component $f_{0 j}^{k}$ varies for the first $k_{0}$ quantile levels, that is, $\left\|\mathbf{d}_{0 j}^{k}\right\| \sim \sqrt{k_{n}}$ for $k=2, \ldots, k_{0}$, and we have $\left\|\overline{\mathbf{d}}_{j}^{k *}\right\| \sim \sqrt{n}$ for $k=2, \ldots, k_{0}$. Since $\omega_{j, k}=\left\|\overline{\mathbf{d}}_{j}^{k *}\right\|^{-1}$ for $k=2, \ldots, k_{0}$,

$$
\begin{aligned}
-\lambda_{n} \sum_{k=2}^{k_{0}} \sum_{j=1}^{p} \sqrt{\frac{k_{n}}{n}} \omega_{j, k}\left\|k_{n}^{1 / 2} \mathbf{u}_{(j, k)}\right\| & =-\lambda_{n} \sum_{k=2}^{k_{0}} \sum_{j=1}^{p} \sqrt{\frac{k_{n}}{n}}\left\|\overline{\mathbf{d}}_{j}^{k *}\right\|^{-1}\left\|k_{n}^{1 / 2} \mathbf{u}_{(j, k)}\right\| \\
& \sim-\frac{\lambda_{n} k_{n}}{n} .
\end{aligned}
$$

Hence, the first term dominates $(2.6)$ when $\frac{\lambda_{n}}{n} \rightarrow 0$ and we complete the proof.

\section{Proof of Theorem 2.3}

Under the model assumption, $\boldsymbol{\theta}_{0}$ can decomposed as $\left(\boldsymbol{\theta}_{0 \mathcal{A}^{c}}^{T}, \mathbf{0}_{\left(K-k_{0}\right) p m_{n}}^{T}\right)^{T}$, where $\boldsymbol{\theta}_{0 \mathcal{A}^{c}} \in \mathbb{R}^{K+k_{0} p m_{n}}$. Denote $\hat{\boldsymbol{\theta}}^{*}=\left(\hat{\boldsymbol{\theta}}_{\mathcal{A}^{c}}^{* T}, \hat{\boldsymbol{\theta}}_{\mathcal{A}}^{* T}\right)^{T}$ and by way of contradiction suppose $\hat{\boldsymbol{\theta}}_{\mathcal{A}}^{* T} \neq \mathbf{0}$. Let $\check{\boldsymbol{\theta}}^{*}$ be a vector constructed by replacing $\hat{\boldsymbol{\theta}}_{\mathcal{A}}^{* T}$ with $\mathbf{0}^{T}$ in $\hat{\boldsymbol{\theta}}^{*}$, that is, $\check{\boldsymbol{\theta}}^{*}=\left(\hat{\boldsymbol{\theta}}_{\mathcal{A}^{c}}^{* T}, \mathbf{0}_{\left(K-k_{0}\right) p m_{n}}^{T}\right)^{T}$. Note that

$$
\begin{aligned}
& Q_{1}^{*}\left(\hat{\boldsymbol{\theta}}^{*}\right)-Q_{1}^{*}\left(\check{\boldsymbol{\theta}}^{*}\right) \\
= & \sum_{k=1}^{K} \sum_{i=1}^{n} \rho_{\tau_{k}}\left(\epsilon_{i k}-R^{k}\left(\mathbf{X}_{i}\right)-\mathbf{Z}_{i k}^{T}\left(\hat{\boldsymbol{\theta}}^{*}-\boldsymbol{\theta}_{0}^{*}\right)\right)-\sum_{k=1}^{K} \sum_{i=1}^{n} \rho_{\tau_{k}}\left(\epsilon_{i k}-R^{k}\left(\mathbf{X}_{i}\right)\right. \\
& \left.-\mathbf{Z}_{i k}^{T}\left(\check{\boldsymbol{\theta}}^{*}-\boldsymbol{\theta}_{0}^{*}\right)\right)+\lambda_{n} \sum_{k=2}^{K} \sum_{j=1}^{p} \sqrt{\frac{k_{n}}{n}} \omega_{j, k}\left[\left\|\hat{\mathbf{d}}_{j}^{k *}\right\|-\left\|\check{\mathbf{d}}_{j}^{k *}\right\|\right] \\
= & \sum_{k=1}^{K} \sum_{i=1}^{n} \rho_{\tau_{k}}\left(\epsilon_{i k}-R^{k}\left(\mathbf{X}_{i}\right)-\mathbf{Z}_{i k}^{T}\left(\hat{\boldsymbol{\theta}}^{*}-\boldsymbol{\theta}_{0}^{*}\right)\right)-\sum_{k=1}^{K} \sum_{i=1}^{n} \rho_{\tau_{k}}\left(\epsilon_{i k}-R^{k}\left(\mathbf{X}_{i}\right)\right. \\
& \left.-\mathbf{Z}_{i k}^{T}\left(\check{\boldsymbol{\theta}}^{*}-\boldsymbol{\theta}_{0}^{*}\right)\right)+\lambda_{n} \sum_{k=k_{0}+1}^{K} \sum_{j=1}^{p} \sqrt{\frac{k_{n}}{n}} \omega_{j, k}\left\|\hat{\mathbf{d}}_{j}^{k *}\right\| .
\end{aligned}
$$


First, we consider the first term in (2.7). Using $\rho_{\tau}(u)-\rho_{\tau}(v) \geq(\tau-I(v \leq$ $0))(u-v)$, similar to the proof of Theorem 1 of Noh et al. (2012), we have

$$
\begin{gathered}
\sum_{k=1}^{K} \sum_{i=1}^{n} \rho_{\tau_{k}}\left(\epsilon_{i k}-R^{k}\left(\mathbf{X}_{i}\right)-\mathbf{Z}_{i k}^{T}\left(\hat{\boldsymbol{\theta}}^{*}-\boldsymbol{\theta}_{0}^{*}\right)\right) \\
-\sum_{k=1}^{K} \sum_{i=1}^{n} \rho_{\tau_{k}}\left(\epsilon_{i k}-R^{k}\left(\mathbf{X}_{i}\right)-\mathbf{Z}_{i k}^{T}\left(\check{\boldsymbol{\theta}}^{*}-\boldsymbol{\theta}_{0}^{*}\right)\right) \\
\geq-\sum_{k=1}^{K} \sum_{i=1}^{n}\left(\tau_{k}-I\left\{\epsilon_{i k}-R^{k}\left(\mathbf{X}_{i}\right)\right.\right. \\
\left.\left.-\mathbf{Z}_{i k}^{T}\left(\left(\check{\boldsymbol{\theta}}_{\mathcal{A}^{c}}^{*}-\boldsymbol{\theta}_{0 \mathcal{A}^{c}}^{*}\right)^{T}, \mathbf{0}^{T}\right)^{T} \leq 0\right\}\right) \mathbf{Z}_{i k}^{T}\left(\mathbf{0}^{T}, \hat{\boldsymbol{\theta}}_{\mathcal{A}}^{* T}\right)^{T} \\
\geq-O_{p}\left(\sqrt{k_{n}}\right)\left\|\hat{\boldsymbol{\theta}}_{\mathcal{A}}^{*}\right\| .
\end{gathered}
$$

Now, we consider the second term in 2.7). Under the model assumption, $\left\|\mathbf{d}_{0 j}^{k *}\right\|=0$ when $k=k_{0}+1, \ldots, K$. By the convergence rate in Theorem 2.1. we have $\left\|\overline{\mathbf{d}}_{j}^{k *}\right\|=O_{p}\left(\sqrt{k_{n}}\right)$ for $k=k_{0}+1, \ldots, K$. Since $\omega_{j, k}=\left\|\overline{\mathbf{d}}_{j}^{k *}\right\|^{-1}$, with probability approaching 1 ,

$$
\begin{aligned}
\lambda_{n} \sum_{k=k_{0}+1}^{K} \sum_{j=1}^{p} \sqrt{\frac{k_{n}}{n}} \omega_{j, k}\left\|\hat{\mathbf{d}}_{j}^{k *}\right\| & =\lambda_{n} \sum_{k=k_{0}+1}^{K} \sum_{j=1}^{p} \sqrt{\frac{k_{n}}{n}}\left\|\overline{\mathbf{d}}_{j}^{k *}\right\|^{-1}\left\|\hat{\mathbf{d}}_{j}^{k *}\right\| \\
& \geq C \frac{\lambda_{n}}{\sqrt{n}}\left\|\hat{\boldsymbol{\theta}}_{\mathcal{A}}^{*}\right\|,
\end{aligned}
$$

where $C$ is a positive constant.

Therefore, we have $Q_{1}^{*}\left(\hat{\boldsymbol{\theta}}^{*}\right)>Q_{1}^{*}\left(\check{\boldsymbol{\theta}}^{*}\right)$, which contradicts that $\hat{\boldsymbol{\theta}}^{*}$ is the minimizer of $Q_{1}^{*}\left(\boldsymbol{\theta}^{*}\right)$.

Proofs of Theorem 2.4 and Theorem 2.5 are almost the same and thus omitted. 


\section{Chapter 3}

\section{Quantile Regression for Additive Coefficient Models in High Dimensions}

Let $\left(Y_{i}, \mathbf{X}_{i}, \mathbf{T}_{i}\right), i=1, \ldots, n$, be random variables/vectors that are independent and identically distributed (i.i.d), where $Y_{i}$ is the response variable and $\mathbf{X}_{i}=\left(X_{i 1}, \ldots, X_{i d}\right)^{\mathrm{T}}$ and $\mathbf{T}_{i}=\left(T_{i 1}, \ldots, T_{i p_{n}}\right)^{\mathrm{T}}$ are predictors. We are interested in regression of $Y_{i}$ on $\left(\mathbf{X}_{i}, \mathbf{T}_{i}\right)$ at a given quantile level $\tau$, $0<\tau<1$. Denote by $Q_{\tau}(Y \mid \mathbf{X}, \mathbf{T})$ the $\tau$-th conditional quantile function of $Y$ given $(\mathbf{X}, \mathbf{T})$,

$$
Q_{\tau}(Y \mid \mathbf{X}, \mathbf{T})=\inf \{y: \mathrm{P}(Y \leq \mid \mathbf{X}, \mathbf{T}) \geq \tau\} .
$$

We consider the quantile additive coefficient model

$$
\begin{aligned}
Q_{\tau}\left(Y_{i} \mid \mathbf{X}_{i}, \mathbf{T}_{i}\right) & =\sum_{l=1}^{p_{n}} \alpha_{l}\left(\mathbf{X}_{i}\right) T_{i l} \\
& =\sum_{l=1}^{p_{n}}\left\{\alpha_{l 0}+\sum_{s=1}^{d} \alpha_{l s}\left(X_{i s}\right)\right\} T_{i l},
\end{aligned}
$$

where the non-parametric function $\alpha_{l s}(\cdot)$ depends on quantile level $\tau$ for $l=1, \ldots, p_{n}$ and $s=1, \ldots, d$, but we omit the dependence in notation for simplicity. To ensure the identification of $\alpha_{l s}$ 's, we assume that 


\section{Chapter 3. Quantile Regression for Additive Coefficient Models}

$\mathrm{E} \alpha_{l s}\left(X_{i s}\right)=0$ for $l=1, \ldots, p_{n}, s=1, \ldots, d$. If $\mathbf{X}_{i}$ is one-dimensional, model (3.1) reduces to the varying coefficient model proposed in Hastie and Tibshirani (1993), in which $\mathbf{X}_{i}$ is typically called an index variable. The varying coefficient models are often used to model nonlinear interactions between the index variable and other predictors. The ACM is motivated from the observation that when $\mathbf{X}_{i}$ is multi-dimensional, varying coefficient models typically suffer from curse of dimensionality due to the need to estimate multivariate non-parametric coefficient functions. The ACM avoids this by using an additive structure for the coefficient functions $\alpha_{l}(\cdot), l=1, \ldots, p_{n}$.

\subsection{Proposed Methodologies}

In this chapter, we consider the above quantile additive coefficient model (3.1) in a high-dimensional setting, which allows $\log \left(p_{n}\right)=O\left(n^{a}\right)$ for some positive constant $a$, while $d$ is fixed. We use the subscript 0 to indicate the true value of the additive coefficient functions. Let $\mathcal{S}:=\left\{l:\left\|\alpha_{0, l}\right\|_{L^{2}} \neq\right.$ $\left.0, l=1, \ldots, p_{n}\right\}$ be the index set of the nonzero additive coefficients. We impose a sparsity condition assuming $|\mathcal{S}|=q_{n}<<p_{n}$, where $q_{n}$ also increases with $n$. Our goal is to identify the true model $\mathcal{S}$ and derive the optimal rate of convergence for $\alpha_{l}, l \in \mathcal{S}$.

We use B-splines to approximate the non-parametric functions $\alpha_{l s}(x)$, $1 \leq l \leq p_{n}, 1 \leq s \leq d$ in model (3.1). Without loss of generality, we suppose that $\mathbf{X}_{i}$ takes value in $[0,1]^{d}$. Let

$$
0=\xi_{0}<\xi_{1}<\ldots<\xi_{k_{n}}<\xi_{k_{n}+1}=1
$$

be a partition of $[0,1]$, where $k_{n}$ is the number of interior knots. We will only use equally spaced knots for simplicity. Let $\left\{B_{j}(x), 1 \leq j \leq J_{n}+1\right\}$ 
be the B-spline basis for the collection of splines with degree $m$, with $\sum_{j} B_{j}(x) \equiv 1$, where $J_{n}+1=k_{n}+m+1$. In this paper, we use cubic splines with $m=3$. Because of the identification condition, we restrict our attention to the subspace of splines defined by $G_{l s}^{0}:=\{g$ : $\left.g(x)=\sum_{j=1}^{J_{n}+1} B_{j}(x) \beta_{l s, j}, \sum_{i=1}^{n} g\left(X_{i s}\right)=0\right\}$ with basis $\left\{B_{s, j}(x)=B_{j}(x)-\right.$ $\left.\frac{1}{n} \sum_{i=1}^{n} B_{j}\left(X_{i s}\right), j=1, \ldots, J_{n}\right\}$. Note that due to the identification constraint, the subspace $G_{l s}^{0}$ is $J_{n}$ dimensional. Using B-spline expansion, we can approximate each additive coefficient function $\alpha_{l}\left(\mathbf{X}_{i}\right)$ in model (3.1) by

$$
\begin{aligned}
\alpha_{l}\left(\mathbf{X}_{i}\right) & \approx \alpha_{l 0}+\sum_{s=1}^{d} \sum_{j=1}^{J_{n}} B_{s, j}\left(X_{i s}\right) \beta_{l s, j} \\
& =\alpha_{l 0}+\sum_{s=1}^{d} \boldsymbol{\Pi}_{s}\left(X_{i s}\right)^{\mathrm{T}} \boldsymbol{\beta}_{l s}
\end{aligned}
$$

where $\boldsymbol{\Pi}_{s}(x)=\left(B_{s, 1}(x), \ldots, B_{s, J_{n}}(x)\right)^{\mathrm{T}}, \boldsymbol{\beta}_{l s}=\left(\beta_{l s, 1}, \ldots, \beta_{l s, J_{n}}\right)^{\mathrm{T}}, s=1, \ldots, d$ and $l=1, \ldots, p_{n}$. Let $\boldsymbol{\beta}_{l}=\left(k_{n}^{1 / 2} \alpha_{l 0}, \boldsymbol{\beta}_{l 1}^{\mathrm{T}}, \ldots, \boldsymbol{\beta}_{l d}^{\mathrm{T}}\right)^{\mathrm{T}}$, for $l=1, \ldots, p_{n}$, and $\boldsymbol{\Pi}\left(\mathbf{X}_{i}\right)=\left(k_{n}^{-1 / 2}, \boldsymbol{\Pi}_{1}\left(X_{i 1}\right)^{\mathrm{T}}, \ldots, \boldsymbol{\Pi}_{d}\left(X_{i d}\right)^{\mathrm{T}}\right)^{\mathrm{T}}$. Then, the conditional quantile in model (3.1) can be approximated by

$$
\begin{aligned}
Q_{\tau}\left(Y_{i} \mid \mathbf{X}_{i}, \mathbf{T}_{i}\right) & \approx \sum_{l=1}^{p_{n}} \boldsymbol{\Pi}\left(\mathbf{X}_{i}\right)^{\mathrm{T}} \boldsymbol{\beta}_{l} T_{i l} \\
& =\boldsymbol{\Pi}\left(\mathbf{X}_{i}, \mathbf{T}_{i}\right)^{\mathrm{T}} \boldsymbol{\beta}
\end{aligned}
$$

where $\boldsymbol{\Pi}\left(\mathbf{X}_{i}, \mathbf{T}_{i}\right)=\left(\boldsymbol{\Pi}\left(\mathbf{X}_{i}\right)^{\mathrm{T}} T_{i 1}, \ldots, \boldsymbol{\Pi}\left(\mathbf{X}_{i}\right)^{\mathrm{T}} T_{i p_{n}}\right)^{\mathrm{T}}, \boldsymbol{\beta}=\left(\boldsymbol{\beta}_{1}^{\mathrm{T}}, \ldots, \boldsymbol{\beta}_{p_{n}}^{\mathrm{T}}\right)^{\mathrm{T}}$.

\subsubsection{Oracle Estimator}

In this section, we consider the oracle estimator for model (3.1). Without loss of generality, we assume the first $q_{n}$ coefficient functions in $\alpha_{0, l}(\cdot)$, $l=1, \ldots, p_{n}$, are nonzero. In other words, we have $\mathcal{S}=\left\{1, \ldots, q_{n}\right\}$. The oracle estimator is obtained assuming that one only uses the predictors $T_{i l}, l \in \mathcal{S}$ (as well as $\mathbf{X}_{i}$ ) in fitting the model (3.1). 


\section{Chapter 3. Quantile Regression for Additive Coefficient Models}

Let $\boldsymbol{\beta}=\left(\boldsymbol{\beta}_{\mathcal{S}}^{\mathrm{T}}, \boldsymbol{\beta}_{\mathcal{S}^{c}}^{\mathrm{T}}\right)^{\mathrm{T}}, \mathbf{T}=\left(\mathbf{T}_{\mathcal{S}}^{\mathrm{T}}, \mathbf{T}_{\mathcal{S}^{c}}^{\mathrm{T}}\right)^{\mathrm{T}}$ and $\boldsymbol{\Pi}\left(\mathbf{X}_{i}, \mathbf{T}_{i}\right)=\left(\boldsymbol{\Pi}_{\mathcal{S}}\left(\mathbf{X}_{i}, \mathbf{T}_{i}\right)^{\mathrm{T}}\right.$, $\left.\boldsymbol{\Pi}_{\mathcal{S}^{c}}\left(\mathbf{X}_{i}, \mathbf{T}_{i}\right)^{\mathrm{T}}\right)^{\mathrm{T}}$, where $\boldsymbol{\beta}_{\mathcal{S}}, \boldsymbol{\Pi}_{\mathcal{S}}\left(\mathbf{X}_{i}, \mathbf{T}_{i}\right) \in \mathbb{R}^{q_{n}\left(1+d J_{n}\right)}$ and $\boldsymbol{\beta}_{\mathcal{S}^{c}}, \boldsymbol{\Pi}_{\mathcal{S}^{c}}\left(\mathbf{X}_{i}, \mathbf{T}_{i}\right) \in$ $\mathbb{R}^{\left(p_{n}-q_{n}\right)\left(1+d J_{n}\right)}$.

Given a quantile level $\tau$, the oracle estimator is $\hat{\boldsymbol{\beta}}=\left(\hat{\boldsymbol{\beta}}_{\mathcal{S}}^{\mathrm{T}}, \mathbf{0}_{\left(p_{n}-q_{n}\right)\left(1+d J_{n}\right)}^{\mathrm{T}}\right)^{\mathrm{T}}$, where $\hat{\boldsymbol{\beta}}_{\mathcal{S}}$ is defined as

$$
\hat{\boldsymbol{\beta}}_{\mathcal{S}}=\underset{\boldsymbol{\beta}_{\mathcal{S}}}{\arg \min } \sum_{i=1}^{n} \rho_{\tau}\left(Y_{i}-\boldsymbol{\Pi}_{\mathcal{S}}\left(\mathbf{X}_{i}, \mathbf{T}_{i}\right)^{\mathrm{T}} \boldsymbol{\beta}_{\mathcal{S}}\right),
$$

$\rho_{\tau}(u)=u(\tau-I(u<0))$ is the quantile loss function, and $I(\cdot)$ is the indicator function.

From Schumaker (2007), there exists a positive constant $M$ such that

$$
\sup _{x \in[0,1]}\left|r_{l s}(x)\right| \leq M k_{n}^{-r},
$$

where $r_{l s}(x)=\boldsymbol{\Pi}_{s}(x)^{\mathrm{T}} \boldsymbol{\beta}_{0, l s}-\alpha_{0, l s}(x)$. Let $\boldsymbol{\beta}_{0}=\left(\boldsymbol{\beta}_{0, \mathcal{S}}^{\mathrm{T}}, \mathbf{0}_{\left(p_{n}-q_{n}\right)\left(1+d J_{n}\right)}^{\mathrm{T}}\right)^{\mathrm{T}}$, where $\boldsymbol{\beta}_{0, \mathcal{S}}=\left(\boldsymbol{\beta}_{0,1}^{\mathrm{T}}, \ldots, \boldsymbol{\beta}_{0, q_{n}}^{\mathrm{T}}\right)^{\mathrm{T}}, \boldsymbol{\beta}_{0, l}=\left(k_{n}^{1 / 2} \alpha_{0 l}, \boldsymbol{\beta}_{0, l 1}^{\mathrm{T}}, \ldots, \boldsymbol{\beta}_{0, l d}^{\mathrm{T}}\right)^{\mathrm{T}}, l \in \mathcal{S}$. Now, we consider the optimization problem in 3.2 rewritten as

$$
\hat{\boldsymbol{\beta}}_{\mathcal{S}}=\underset{\boldsymbol{\beta}_{\mathcal{S}}}{\arg \min } \sum_{i=1}^{n} \rho_{\tau}\left(\epsilon_{i}-R_{i, \mathcal{S}}-\boldsymbol{\Pi}_{\mathcal{S}}\left(\mathbf{X}_{i}, \mathbf{T}_{i}\right)^{\mathrm{T}}\left(\boldsymbol{\beta}_{\mathcal{S}}-\boldsymbol{\beta}_{0, \mathcal{S}}\right)\right),
$$

where $\epsilon_{i}=Y_{i}-\sum_{l=1}^{q_{n}} \alpha_{0, l}\left(\mathbf{X}_{i}\right) T_{i l}, R_{i, \mathcal{S}}:=\sum_{l \in \mathcal{S}} \sum_{s=1}^{d} r_{l s}\left(X_{i s}\right) T_{i l}$. Denote by $\hat{\alpha}_{l}$ the oracle estimator of $\alpha_{l}$ defined through $\hat{\boldsymbol{\beta}}_{l}$ for $l \in \mathcal{S}$, at a given quantile level $\tau$.

\subsubsection{Group SCAD Penalized Estimator}

In this section, we investigate model selection for model (3.1) without information about $\mathcal{S}$. We consider a group SCAD penalized quantile loss

$$
L_{n}(\boldsymbol{\beta}):=\sum_{i=1}^{n} \rho_{\tau}\left(Y_{i}-\boldsymbol{\Pi}\left(\mathbf{X}_{i}, \mathbf{T}_{i}\right)^{\mathrm{T}} \boldsymbol{\beta}\right)+n \sum_{l=1}^{p_{n}} p_{\lambda_{n}}\left(\left\|\boldsymbol{\beta}_{l}\right\|\right),
$$


where

$$
\begin{aligned}
p_{\lambda_{n}}(t)= & \lambda_{n} t I\left(0 \leq t<\lambda_{n}\right)+\frac{a \lambda_{n} t-\left(t^{2}+\lambda_{n}^{2}\right) / 2}{a-1} I\left(\lambda_{n} \leq t \leq a \lambda_{n}\right) \\
& +\frac{(a+1) \lambda_{n}^{2}}{2} I\left(t>a \lambda_{n}\right),
\end{aligned}
$$

for some $a>2, t>0$, and $\lambda_{n} \geq 0$ is a tuning parameter controlling the degree of penalization. Note that the penalized quantile loss function $L_{n}(\boldsymbol{\beta})$ is non-differentiable and non-convex. For theoretical derivations later, we follow the strategy in Wang et al. (2012) and rewrite the objective function $L_{n}(\boldsymbol{\beta})$ as a difference of two convex functions, that is,

$$
L_{n}(\boldsymbol{\beta}):=G_{n}(\boldsymbol{\beta})-H_{n}(\boldsymbol{\beta}),
$$

where $G_{n}(\boldsymbol{\beta}), H_{n}(\boldsymbol{\beta})$ are convex functions,

$$
G_{n}(\boldsymbol{\beta})=\sum_{i=1}^{n} \rho_{\tau}\left(Y_{i}-\boldsymbol{\Pi}\left(\mathbf{X}_{i}, \mathbf{T}_{i}\right)^{\mathrm{T}} \boldsymbol{\beta}\right)+n \lambda_{n} \sum_{l=1}^{p_{n}}\left\|\boldsymbol{\beta}_{l}\right\|,
$$

and

$$
\begin{aligned}
H_{n}(\boldsymbol{\beta})= & n \sum_{l=1}^{p_{n}}\left\{\frac{\left\|\boldsymbol{\beta}_{l}\right\|^{2}-2 \lambda_{n}\left\|\boldsymbol{\beta}_{l}\right\|+\lambda_{n}^{2}}{2(a-1)} I\left(\lambda_{n} \leq\left\|\boldsymbol{\beta}_{l}\right\| \leq a \lambda_{n}\right)\right. \\
& \left.+\left(\lambda_{n}\left\|\boldsymbol{\beta}_{l}\right\|-2(a+1) \lambda_{n}^{2} / 2\right) I\left(\left\|\boldsymbol{\beta}_{l}\right\|>a \lambda_{n}\right)\right\} .
\end{aligned}
$$

Since the function $G_{n}(\boldsymbol{\beta})$ is not differentiable everywhere, we define the subdifferential of $G_{n}(\boldsymbol{\beta})$ at $\boldsymbol{\beta}$ as $\partial G_{n}(\boldsymbol{\beta})=\left(\partial G_{n}(\boldsymbol{\beta}) / \partial \boldsymbol{\beta}_{1}^{\mathrm{T}}, \ldots, \partial G_{n}(\boldsymbol{\beta}) / \partial \boldsymbol{\beta}_{p_{n}}^{\mathrm{T}}\right)^{\mathrm{T}} \in$ $\mathcal{R}^{p_{n}\left(1+d J_{n}\right)}$, where

$$
\begin{aligned}
\partial G_{n}(\boldsymbol{\beta}) / \partial \boldsymbol{\beta}_{l}= & -\tau \sum_{i=1}^{n} T_{i l} I\left(Y_{i}-\boldsymbol{\Pi}\left(\mathbf{X}_{i}, \mathbf{T}_{i}\right)^{\mathrm{T}} \boldsymbol{\beta}>0\right) \boldsymbol{\Pi}\left(\mathbf{X}_{i}\right) \\
& +(1-\tau) \sum_{i=1}^{n} T_{i l} I\left(Y_{i}-\boldsymbol{\Pi}\left(\mathbf{X}_{i}, \mathbf{T}_{i}\right)^{\mathrm{T}} \boldsymbol{\beta}<0\right) \boldsymbol{\Pi}\left(\mathbf{X}_{i}\right) \\
& -\sum_{i=1}^{n} a_{i l} T_{i l} \boldsymbol{\Pi}\left(\mathbf{X}_{i}\right)+n \lambda_{n} \boldsymbol{\beta}_{l} /\left\|\boldsymbol{\beta}_{l}\right\|
\end{aligned}
$$




\section{Chapter 3. Quantile Regression for Additive Coefficient Models}

$$
:=\boldsymbol{\nu}_{l}(\boldsymbol{\beta})+n \lambda_{n} \boldsymbol{\beta}_{l} /\left\|\boldsymbol{\beta}_{l}\right\|
$$

$l=1, \ldots, p_{n}$, if $\boldsymbol{\beta}_{l} \neq \mathbf{0}$, and $\boldsymbol{\beta}_{l} /\left\|\boldsymbol{\beta}_{l}\right\|$ in the above is replaced by any vector with norm bounded by 1 if $\boldsymbol{\beta}_{l}=\mathbf{0}$. In the displayed equation above, $a_{i l}=0$ if $Y_{i}-\boldsymbol{\Pi}\left(\mathbf{X}_{i}, \mathbf{T}_{i}\right)^{\mathrm{T}} \boldsymbol{\beta} \neq 0$, and $a_{i l} \in[\tau-1, \tau]$ otherwise. The function $H_{n}(\boldsymbol{\beta})$ is differentiable everywhere and we denote by $\partial H_{n}(\boldsymbol{\beta})=\left(\partial H_{n}(\boldsymbol{\beta}) / \partial \boldsymbol{\beta}_{1}^{\mathrm{T}}, \ldots\right.$, $\left.\partial H_{n}(\boldsymbol{\beta}) / \partial \boldsymbol{\beta}_{p_{n}}^{\mathrm{T}}\right)^{\mathrm{T}} \in \mathcal{R}^{p_{n}\left(1+d J_{n}\right)}$ the differential of $H_{n}(\boldsymbol{\beta})$ at $\boldsymbol{\beta}$, where

$$
\partial H_{n}(\boldsymbol{\beta}) / \partial \boldsymbol{\beta}_{l}= \begin{cases}0, & 0 \leq\left\|\boldsymbol{\beta}_{l}\right\|<\lambda_{n} \\ n \frac{\boldsymbol{\beta}_{l}-\lambda_{n} \boldsymbol{\beta}_{l} /\left\|\boldsymbol{\beta}_{l}\right\|}{a-1}, & \lambda_{n} \leq\left\|\boldsymbol{\beta}_{l}\right\| \leq a \lambda_{n} \\ n \lambda_{n} \boldsymbol{\beta}_{l} /\left\|\boldsymbol{\beta}_{l}\right\|, & \left\|\boldsymbol{\beta}_{l}\right\|>a \lambda_{n}\end{cases}
$$

$l=1, \ldots, p_{n}$.

\subsubsection{Computation}

We combine the MM algorithm for quantile regression and an approximation based on local linear approximation (LLA) to solve the penalized quantile loss function (3.4). More specifically, we first approximate the penalty function $p_{\lambda_{n}}\left(\left\|\boldsymbol{\beta}_{l}\right\|\right)$ by LLA. We use

$$
p_{\lambda_{n}}\left(\left\|\boldsymbol{\beta}_{l}\right\|\right) \approx p_{\lambda_{n}}\left(\left\|\boldsymbol{\beta}_{l}^{(t)}\right\|\right)+p_{\lambda_{n}}^{\prime}\left(\left\|\boldsymbol{\beta}_{l}^{(t)}\right\|\right)\left(\left\|\boldsymbol{\beta}_{l}\right\|-\left\|\boldsymbol{\beta}_{l}^{(t)}\right\|\right),
$$

for the current estimate of $\boldsymbol{\beta}_{l}, \boldsymbol{\beta}_{l}^{(t)}$, in the $t$-th iteration, $t=0,1, \ldots$ Hence, the group SCAD penalized quantile loss function in $(3.4)$ in each iteration $t$ reduces to

$$
\sum_{i=1}^{n} \rho_{\tau}\left(Y_{i}-\boldsymbol{\Pi}\left(\mathbf{X}_{i}, \mathbf{T}_{i}\right)^{\mathrm{T}} \boldsymbol{\beta}\right)+n \sum_{l=1}^{p_{n}} p_{\lambda_{n}}^{\prime}\left(\left\|\boldsymbol{\beta}_{l}^{(t)}\right\|\right)\left\|\boldsymbol{\beta}_{l}\right\| .
$$

For the quantile loss function, we use the majorization

$$
\sum_{i} \xi_{\epsilon}\left(r_{i} \mid r_{i}^{(t)}\right)=\sum_{i} \frac{1}{4}\left\{\frac{r_{i}^{2}}{\epsilon+\left|r_{i}^{(t)}\right|}+(4 \tau-2) r_{i}+c\right\},
$$


to replace the non-differentiable quantile loss function $\rho_{\tau}(r)$ in (3.7) in each iteration, where $r_{i}^{(t)}=Y_{i}-\boldsymbol{\Pi}\left(\mathbf{X}_{i}, \mathbf{T}_{i}\right)^{\mathrm{T}} \boldsymbol{\beta}^{(t)}$ is the residual at the $t$-th iteration, $t=1,2, \ldots$ and $r_{i}=Y_{i}-\boldsymbol{\Pi}\left(\mathbf{X}_{i}, \mathbf{T}_{i}\right)^{\mathrm{T}} \boldsymbol{\beta}, \epsilon$ is a small scalar chosen to be $10^{-2}$ here, and $c$ is a constant that does not depend on $\boldsymbol{\beta}$ and thus can be ignored during optimization. In each iteration, we use $\mathrm{R}$ package gglasso (Yang and Zou (2015)) to solve the optimization problem 3.7) with quantile loss function replaced by (3.8). Finally, both $k_{n}$ and $\lambda_{n}$ are selected by ten-fold cross-validation using the quantile loss in all our numerical examples, and following Fan and Li (2001), we take $a=3.7$ in the SCAD penalty (3.5).

\subsection{Theoretical Results}

The following conditions are assumed for theoretical results.

(C1) (Condition on non-parametric functions) $\mathrm{E} \alpha_{0, l s}\left(X_{i s}\right)=0$ and $\alpha_{0, l s} \in$ $\mathcal{H}_{r}$ for $l=1, \ldots, p_{n}, s=1, \ldots, d$, where $\mathcal{H}_{r}$ is the collection of functions, $f$, on $[0,1]$ such that the $v$-th order derivative satisfies the Hölder condition of order $\gamma$ with $r=\gamma+v>\frac{1}{2}, v$ is a positive integer and $\gamma \in(0,1]$, that is,

$$
\left|f^{(v)}(u)-f^{(v)}\left(u^{\prime}\right)\right| \leq C_{1}\left|u-u^{\prime}\right|^{\gamma}, \text { for } u, u^{\prime} \in[0,1]
$$

where $C_{1}$ is a positive constant.

(C2) (Condition on true model size) $q_{n}=O\left(n^{c_{1}}\right)$ for some $0<c_{1}<1 / 4$.

(C3) (Condition on predictors $\mathbf{X}) \mathbf{X}=\left(X_{1}, \ldots, X_{d}\right)$ has an absolutely continuous density supported on $[0,1]^{d}$, and is bounded and bounded away from zero. 


\section{Chapter 3. Quantile Regression for Additive Coefficient Models}

(C4) (Condition on predictors $\mathbf{T}$ ) There exist positive constants $c_{2}$ and $c_{3}$ such that $c_{2} \mathbf{I}_{q_{n}} \leq \mathrm{E}\left(\mathbf{T}_{\mathcal{S}} \mathbf{T}_{\mathcal{S}}^{\mathrm{T}} \mid \mathbf{X}\right) \leq c_{3} \mathbf{I}_{q_{n}}$, where $\mathbf{I}_{q_{n}}$ is a $q_{n} \times q_{n}$ identity matrix and $\mathbf{T}_{\mathcal{S}}=\left(T_{1}, \ldots, T_{q_{n}}\right)^{\mathrm{T}}$.

(C5) (Condition on the number of knots) $k_{n} \sim n^{1 /(2 r+1)}$.

(C6) (Condition on the random error $\boldsymbol{\epsilon}$ ) Let $\epsilon_{i}:=Y_{i}-\sum_{l=1}^{p_{n}} \alpha_{0, l}\left(\mathbf{X}_{i}\right) T_{i l}, i=$ $1, \ldots, n$. We have $\mathrm{P}\left(\epsilon_{i} \leq 0 \mid \mathbf{X}_{i}, \mathbf{T}_{i}\right)=\tau$. The conditional distribution of $\epsilon_{i}$ given $\left(\mathbf{X}_{i}, \mathbf{T}_{i}\right)$ has a density function $f_{i}\left(\cdot \mid \mathbf{X}_{i}, \mathbf{T}_{i}\right)$ with $0<c<$ $f_{i}\left(0 \mid \mathbf{X}_{i}, \mathbf{T}_{i}\right)<C<\infty$ for some constants $c, C$. The density function $f_{i}(\cdot \mid \mathbf{X}, \mathbf{T})$ has a bounded first derivative in a neighborhood of zero, uniformly over $i$.

(C7) (Condition on the signal strength) There exists $\frac{1}{2 r+1}<c_{4}<1$ and a positive constant $c_{5}$ such that $\min _{l \in \mathcal{S}}\left\|\alpha_{0, l}\right\|_{L^{2}} \geq c_{5}\left(n^{-\left(1-c_{4}\right) / 2} k_{n}^{1 / 2}+\right.$ $\left.k_{n}^{-r}\right)$.

\subsubsection{Oracle Estimator}

Theorem 3.1. Under conditions (C1)-(C6), we have

$$
\frac{1}{n} \sum_{l \in \mathcal{S}} \sum_{i=1}^{n}\left(\hat{\alpha}_{l}\left(\mathbf{X}_{i}\right)-\alpha_{0, l}\left(\mathbf{X}_{i}\right)\right)^{2}=O_{p}\left(q_{n}\left(k_{n} n^{-1}+k_{n}^{-2 r}\right)\right)
$$

\subsubsection{Group SCAD Penalized Estimator}

Let $\mathcal{E}\left(\lambda_{n}\right)$ be the set of local minima of the penalized objective function $L_{n}(\boldsymbol{\beta})$ for a given tuning parameter $\lambda_{n}$. We have the following theoretical result.

Theorem 3.2. Under Conditions (C1)-(C7), if $\lambda_{n}=o\left(n^{-\left(1-c_{4}\right) / 2} k_{n}^{1 / 2}\right)$, $q_{n} k_{n}^{3 / 2} n^{-1 / 2}=o\left(\lambda_{n}\right)$, and $n \lambda_{n} /\left(k_{n}^{1 / 2} \log \left(p_{n}\right)\right) \rightarrow \infty$, the oracle estimator 
$\hat{\boldsymbol{\beta}}$ satisfies

$$
\mathrm{P}\left(\hat{\boldsymbol{\beta}} \in \mathcal{E}\left(\lambda_{n}\right)\right) \rightarrow 1, \text { as } n \rightarrow \infty
$$

\subsection{Numerical Results}

\subsubsection{Simulation Study}

In this section, we demonstrate the performance of the proposed method by simulation studies.

Example 3.1. Consider the following quantile additive coefficient model

$$
Y_{i}=\sum_{l=1}^{p_{n}}\left\{\alpha_{l 0}+\sum_{s=1}^{2} \alpha_{l s}\left(X_{i s}\right)\right\} T_{i l}+\left(1+\kappa\left(\left|T_{i 1}\right|+\left|T_{i 4}\right|+\left|X_{i 2}\right|\right) / 2\right) \epsilon_{i},
$$

where $\mathbf{X}=\left(X_{1}, X_{2}\right)^{\mathrm{T}}$ is uniformly distributed on $[0,1]^{2}$ independent of $\mathbf{T}$, and $\mathbf{T}=\left(T_{1}, \ldots, T_{p_{n}}\right)^{\mathrm{T}}$ is generated from a multivariate normal distribution with mean 0 and $\operatorname{Cov}\left(T_{i l_{1}}, T_{i l_{2}}\right)=0.5^{\left|l_{1}-l_{2}\right|}, 1 \leq l_{1}, l_{2} \leq p_{n}$. The nonzero non-parametric functions are $\alpha_{4,0}=1, \alpha_{4,1}(x)=5 x(1-x)$, $\alpha_{4,2}(x)=\sin (\pi x), \alpha_{15,0}=2, \alpha_{15,1}(x)=\cos (\pi x), \alpha_{15,2}(x)=2 x$. The error $\epsilon_{i}$ is independent of $(\mathbf{X}, \mathbf{T})$ and follows either a standard normal distribution or a Student's t distribution with scale parameter $1 / 3$ and degrees of freedom 3. The errors are shifted by the location parameter $-q_{\tau}$, where $q_{\tau}$ is the $\tau$-th quantile of the standard normal distribution or the Student's t distribution, which makes sure that the $\tau$-th quantile of $\epsilon_{i}$ is 0 . We simulate 200 datasets with $n=100,200,300, p_{n}=100,300,600$, and $\kappa=0,1$, for different quantile levels. When $\tau=0.5$, we also compare the quantile (median) regression method with the group SCAD penalized least squares method (by replacing the quantile loss in (3.4) with the quadratic loss).

To assess the performance of simulations, we use the following criteria. 


\section{Chapter 3. Quantile Regression for Additive Coefficient Models}

1. Average Integrated Squared Error (AISE): Denote by $\hat{\alpha}_{j, l s}$ the penalized quantile estimate in the $j$-th replication for non-parametric function $\alpha_{l s}$. We define the integrated squared error (ISE) for $\hat{\alpha}_{j, l s}$ as

$$
\operatorname{ISE}\left(\hat{\alpha}_{j, l s}\right)=\frac{1}{n_{\text {grid }}} \sum_{t=1}^{n_{\text {grid }}}\left(\hat{\alpha}_{j, l s}\left(x_{t}\right)-\alpha_{l s}\left(x_{t}\right)\right)^{2},
$$

over an equally spaced 1000 points $\left\{x_{1}, \ldots, x_{n_{\text {grid }}}\right\}, n_{\text {grid }}=1000$ on $[0,1]$. Over 200 replications, the AISE for $\hat{\alpha}_{l s}$ is

$$
\operatorname{AISE}\left(\hat{\alpha}_{l s}\right)=\frac{1}{200} \sum_{j=1}^{200} \operatorname{ISE}\left(\hat{\alpha}_{j, l s}\right) .
$$

2. False Detection (FD): average number of nonzero variables incorrectly included in the estimated model.

3. True Detection (TD): average number of nonzero variables correctly included in the estimated model.

4. True Model (TM): proportion of times the true model is exactly identified.

First we consider model (3.9) with $\kappa=0$ (homogeneous error). Simulation results are summarized in Tables 3.1 3.4. Table 3.1 and Table 3.2 correspond to cases with normal errors, while Table 3.3 and Table 3.4 correspond to cases with Students' t error. We compare the proposed group SCAD penalized quantile regression (PQR) with group SCAD penalized least square regression (PLS) when $\tau=0.5$. Table 3.1 shows that PQR is worse than PLS when the error is normal, while Table 3.3 shows that PQR performs better with heavy-tailed Student's t error, which is as expected. Both PQR and PLS can identify the true model (2 relevant predictors in $\mathbf{T}$ in the true model) with high probabilities. For illustration, some estimated 
non-parametric functions are plotted in Figures 3.1 and 3.2. As the sample size $n$ increases, we get visually better estimators.

Tables 3.5 3.8 report the results when $\kappa=1$ (heterogeneous error). With heterogeneous normal error, at $\tau=0.5$ (Table 3.5), PLS is often, although not always, better than PQR. For Student's t error, it is seen, as before, that PQR is better than PLS (Table 3.7). Some estimated non-parametric functions are plotted in Figures 3.3 and 3.4 .

\subsubsection{Genome Wide Association Study}

In this section, we apply the proposed method to a genome wide association study (GWAS). The sheep dataset was first studied by Zhang et al. (2013) and it can be found on gene expression omnibus (http://www.ncbi.nlm.nih. gov/gds) with series number GSE46231. It contains 329 samples, 13 traits 


\section{Chapter 3. Quantile Regression for Additive Coefficient Models in High Dimensions}

Table 3.1: Simultaion results for $\kappa=0$ when $\epsilon_{i}$ follows a standard normal distribution and $\tau=0.5$. Standard deviations computed based on 200 repetitions are shown in the parentheses.

\begin{tabular}{|c|c|c|c|c|c|c|c|c|c|c|}
\hline \multirow[b]{2}{*}{ Method } & \multirow[b]{2}{*}{$\left(n, p_{n}\right)$} & \multicolumn{6}{|c|}{$\sqrt{\mathrm{AISE}}$} & \multirow[b]{2}{*}{ FD } & \multirow[b]{2}{*}{ TD } & \multirow[b]{2}{*}{$\mathrm{TM}$} \\
\hline & & $\alpha_{4,0}$ & $\alpha_{4,1}$ & $\alpha_{4,2}$ & $\alpha_{15,0}$ & $\alpha_{15,1}$ & $\alpha_{15,2}$ & & & \\
\hline \multirow[t]{18}{*}{ PQR } & $(100,100)$ & 0.0984 & 0.1712 & 0.1614 & 0.1100 & 0.1663 & 0.1571 & 0 & 2 & 1 \\
\hline & & $(0.0778)$ & $(0.0850)$ & $(0.0744)$ & $(0.0940)$ & $(0.0827)$ & $(0.0854)$ & (0) & (0) & - \\
\hline & $(100,300)$ & 0.1053 & 0.1614 & 0.1709 & 0.1254 & 0.1558 & 0.1542 & 0.005 & 2 & 0.995 \\
\hline & & $(0.0797)$ & $(0.0698)$ & $(0.0840)$ & $(0.0928)$ & $(0.0693)$ & $(0.0845)$ & $(0.070)$ & (0) & - \\
\hline & $(100,600)$ & 0.1094 & 0.1657 & 0.1629 & 0.1138 & 0.1679 & 0.1518 & 0 & 2 & 1 \\
\hline & & $(0.0824)$ & $(0.0704)$ & $(0.0753)$ & $(0.0903)$ & $(0.0734)$ & $(0.0849)$ & (0) & $(0)$ & - \\
\hline & $(200,100)$ & 0.0755 & 0.1197 & 0.1200 & 0.0803 & 0.1198 & 0.1045 & 0 & 2 & 1 \\
\hline & & $(0.0601)$ & $(0.0425)$ & $(0.0483)$ & $(0.0570)$ & $(0.0442)$ & $(0.0548)$ & (0) & (0) & - \\
\hline & $(200,300)$ & 0.0786 & 0.1186 & 0.1261 & 0.0881 & 0.1205 & 0.1006 & 0 & 2 & 1 \\
\hline & & $(0.0581)$ & $(0.0423)$ & $(0.0512)$ & $(0.0627)$ & $(0.0461)$ & $(0.0497)$ & (0) & (0) & - \\
\hline & $(200,600)$ & 0.0750 & 0.1298 & 0.1228 & 0.0872 & 0.1203 & 0.1052 & 0 & 2 & 1 \\
\hline & & $(0.0540)$ & $(0.0480)$ & $(0.0497)$ & $(0.0637)$ & $(0.0413)$ & $(0.0557)$ & (0) & $(0)$ & - \\
\hline & $(300,100)$ & 0.0628 & 0.1109 & 0.1045 & 0.0609 & 0.1065 & 0.0851 & 0 & 2 & 1 \\
\hline & & $(0.0441)$ & $(0.0361)$ & $(0.0375)$ & $(0.0468)$ & $(0.0365)$ & $(0.0425)$ & (0) & $(0)$ & - \\
\hline & $(300,300)$ & 0.0628 & 0.1132 & 0.1041 & 0.0749 & 0.1069 & 0.0826 & 0 & 2 & 1 \\
\hline & & $(0.0446)$ & $(0.0379)$ & $(0.0340)$ & $(0.0540)$ & $(0.0347)$ & $(0.0429)$ & (0) & (0) & - \\
\hline & $(300,600)$ & 0.0621 & 0.1123 & 0.1047 & 0.0676 & 0.1077 & 0.0820 & 0 & 2 & 1 \\
\hline & & $(0.0469)$ & $(0.0378)$ & $(0.0333)$ & $(0.0493)$ & $(0.0341)$ & $(0.0404)$ & (0) & (0) & - \\
\hline \multirow[t]{18}{*}{ PLS } & $(100,100)$ & 0.0917 & 0.1548 & 0.1413 & 0.1146 & 0.1569 & 0.1424 & 0.005 & 2 & 0.995 \\
\hline & & $(0.0729)$ & $(0.0670)$ & $(0.0602)$ & $(0.0889)$ & $(0.0738)$ & $(0.0700)$ & $(0.070)$ & (0) & - \\
\hline & $(100,300)$ & 0.0957 & 0.1508 & 0.1601 & 0.1181 & 0.1490 & 0.1441 & 0 & 2 & 1 \\
\hline & & $(0.0771)$ & $(0.0713)$ & $(0.0764)$ & $(0.0908)$ & $(0.0655)$ & $(0.0800)$ & (0) & (0) & - \\
\hline & $(100,600)$ & 0.1037 & 0.1522 & 0.1490 & 0.1081 & 0.1570 & 0.1381 & 0.005 & 2 & 0.995 \\
\hline & & $(0.0778)$ & $(0.0640)$ & $(0.0659)$ & $(0.0850)$ & $(0.0689)$ & $(0.0759)$ & $(0.070)$ & (0) & - \\
\hline & $(200,100)$ & 0.0719 & 0.1099 & 0.1123 & 0.0765 & 0.1110 & 0.0937 & 0 & 2 & 1 \\
\hline & & $(0.0555)$ & $(0.0401)$ & $(0.0457)$ & $(0.0592)$ & $(0.0395)$ & $(0.0480)$ & (0) & (0) & - \\
\hline & $(200,300)$ & 0.0708 & 0.1102 & 0.1103 & 0.0868 & 0.1110 & 0.0921 & 0 & 2 & 1 \\
\hline & & $(0.0517)$ & $(0.0363)$ & $(0.0454)$ & $(0.0606)$ & $(0.0401)$ & $(0.0475)$ & (0) & (0) & - \\
\hline & $(200,600)$ & 0.0698 & 0.1203 & 0.1139 & 0.0821 & 0.1087 & 0.0956 & 0 & 2 & 1 \\
\hline & & $(0.0510)$ & $(0.0448)$ & $(0.0464)$ & $(0.0623)$ & $(0.0388)$ & $(0.0496)$ & (0) & (0) & - \\
\hline & $(300,100)$ & 0.0560 & 0.1010 & 0.0962 & 0.0571 & 0.0971 & 0.0762 & 0 & 2 & 1 \\
\hline & & $(0.0429)$ & $(0.0320)$ & $(0.0311)$ & $(0.0480)$ & $(0.0299)$ & $(0.0386)$ & (0) & (0) & - \\
\hline & $(300,300)$ & 0.0597 & 0.1010 & 0.0941 & 0.0635 & 0.0982 & 0.0718 & 0 & 2 & 1 \\
\hline & & $(0.0401)$ & $(0.0347)$ & $(0.0343)$ & $(0.0496)$ & $(0.0310)$ & $(0.0378)$ & (0) & $(0)$ & - \\
\hline & $(300,600)$ & 0.0560 & 0.1015 & 0.0956 & 0.0629 & 0.0994 & 0.0772 & 0 & 2 & 1 \\
\hline & & $(0.0473)$ & $(0.0319)$ & $(0.0311)$ & $(0.0478)$ & $(0.0336)$ & $(0.0368)$ & (0) & $(0)$ & - \\
\hline
\end{tabular}


Table 3.2: Simulation results for $\kappa=0$ when $\epsilon_{i}$ follows a standard normal distribution and $\tau=0.7,0.9$. Standard deviations are shown in the parentheses.

\begin{tabular}{|c|c|c|c|c|c|c|c|c|c|}
\hline \multirow[b]{2}{*}{$\left(n, \tau, p_{n}\right)$} & \multicolumn{6}{|c|}{$\sqrt{\mathrm{AISE}}$} & \multirow[b]{2}{*}{ FD } & \multirow[b]{2}{*}{ TD } & \multirow[b]{2}{*}{$\mathrm{TM}$} \\
\hline & $\alpha_{4,0}$ & $\alpha_{4,1}$ & $\alpha_{4,2}$ & $\alpha_{15,0}$ & $\alpha_{15,1}$ & $\alpha_{15,2}$ & & & \\
\hline \multirow{2}{*}{$(100,0.7,100)$} & 0.1007 & 0.1737 & 0.1685 & 0.1310 & 0.1766 & 0.1557 & 0 & 2 & 1 \\
\hline & $(0.0867)$ & $(0.0817)$ & $(0.0758)$ & $(0.0982)$ & $(0.0825)$ & $(0.0872)$ & $(0)$ & (0) & - \\
\hline \multirow[t]{2}{*}{$(100,0.7,300)$} & 0.1097 & 0.1695 & 0.1783 & 0.1266 & 0.1660 & 0.1573 & 0.010 & 2 & 0.990 \\
\hline & $(0.0863)$ & $(0.0771)$ & $(0.0795)$ & $(0.0909)$ & $(0.0743)$ & $(0.0887)$ & $(0.099)$ & (0) & - \\
\hline \multirow[t]{2}{*}{$(100,0.7,600)$} & 0.1141 & 0.1720 & 0.1712 & 0.1208 & 0.1707 & 0.1498 & 0 & 2 & 1 \\
\hline & $(0.0816)$ & $(0.0726)$ & $(0.0751)$ & $(0.0959)$ & $(0.0694)$ & $(0.0851)$ & $(0)$ & (0) & - \\
\hline \multirow[t]{2}{*}{$(100,0.9,100)$} & 0.1256 & 0.1963 & 0.1879 & 0.1483 & 0.2033 & 0.1684 & 0.005 & 2 & 0.995 \\
\hline & $(0.1041)$ & $(0.1032)$ & $(0.0917)$ & $(0.1105)$ & $(0.0897)$ & $(0.0889)$ & $(0.070)$ & (0) & - \\
\hline \multirow[t]{2}{*}{$(100,0.9,300)$} & 0.1313 & 0.2051 & 0.1999 & 0.1420 & 0.1913 & 0.1891 & 0.005 & 2 & 0.995 \\
\hline & $(0.1007)$ & $(0.1004)$ & $(0.0917)$ & $(0.1076)$ & $(0.0858)$ & $(0.1044)$ & $(0.070)$ & (0) & - \\
\hline \multirow[t]{2}{*}{$(100,0.9,600)$} & 0.1211 & 0.1946 & 0.1934 & 0.1386 & 0.1906 & 0.1697 & 0.005 & 2 & 0.995 \\
\hline & $(0.0928)$ & $(0.0862)$ & $(0.0868)$ & $(0.1061)$ & $(0.0944)$ & $(0.0894)$ & $(0.070)$ & $(0)$ & - \\
\hline \multirow[t]{2}{*}{$(200,0.7,100)$} & 0.0784 & 0.1261 & 0.1281 & 0.0849 & 0.1271 & 0.1053 & 0 & 2 & 1 \\
\hline & $(0.0612)$ & $(0.0455)$ & $(0.0535)$ & $(0.0612)$ & $(0.0453)$ & $(0.0532)$ & (0) & (0) & - \\
\hline \multirow[t]{2}{*}{$(200,0.7,300)$} & 0.0783 & 0.1258 & 0.1218 & 0.0940 & 0.1293 & 0.1044 & 0 & 2 & 1 \\
\hline & $(0.0612)$ & $(0.0435)$ & $(0.0494)$ & $(0.0652)$ & $(0.0482)$ & $(0.0562)$ & (0) & (0) & - \\
\hline \multirow[t]{2}{*}{$(200,0.7,600)$} & 0.0792 & 0.1343 & 0.1281 & 0.0869 & 0.1221 & 0.1114 & 0.010 & 2 & 0.995 \\
\hline & $(0.0572)$ & $(0.0506)$ & $(0.0527)$ & $(0.0640)$ & $(0.0460)$ & $(0.0559)$ & $(0.141)$ & (0) & - \\
\hline \multirow[t]{2}{*}{$(200,0.9,100)$} & 0.0895 & 0.1418 & 0.1516 & 0.1009 & 0.1450 & 0.1268 & 0 & 2 & 1 \\
\hline & $(0.0705)$ & $(0.0529)$ & $(0.0664)$ & $(0.0726)$ & $(0.0570)$ & $(0.0636)$ & (0) & (0) & - \\
\hline \multirow[t]{2}{*}{$(200,0.9,300)$} & 0.0884 & 0.1509 & 0.1449 & 0.1045 & 0.1470 & 0.1265 & 0 & 2 & 1 \\
\hline & $(0.0688)$ & $(0.0580)$ & $(0.0570)$ & $(0.0781)$ & $(0.0615)$ & $(0.0721)$ & (0) & (0) & - \\
\hline \multirow[t]{2}{*}{$(200,0.9,600)$} & 0.0950 & 0.1539 & 0.1468 & 0.0988 & 0.1441 & 0.1236 & 0 & 2 & 1 \\
\hline & $(0.0688)$ & $(0.0627)$ & $(0.0635)$ & $(0.0718)$ & $(0.0577)$ & $(0.0706)$ & $(0)$ & $(0)$ & - \\
\hline \multirow[t]{2}{*}{$(300,0.7,100)$} & 0.0629 & 0.1152 & 0.1107 & 0.0682 & 0.1095 & 0.0813 & 0 & 2 & 1 \\
\hline & $(0.0447)$ & $(0.0339)$ & $(0.0379)$ & $(0.0515)$ & $(0.0346)$ & $(0.0413)$ & (0) & (0) & - \\
\hline \multirow[t]{2}{*}{$(300,0.7,300)$} & 0.0650 & 0.1156 & 0.1066 & 0.0774 & 0.1065 & 0.0827 & 0 & 2 & 1 \\
\hline & $(0.0431)$ & $(0.0391)$ & $(0.0385)$ & $(0.0530)$ & $(0.0358)$ & $(0.0405)$ & (0) & (0) & - \\
\hline \multirow[t]{2}{*}{$(300,0.7,600)$} & 0.0658 & 0.1165 & 0.1076 & 0.0696 & 0.1102 & 0.0852 & 0 & 2 & 1 \\
\hline & $(0.0501)$ & $(0.0397)$ & $(0.0358)$ & $(0.0514)$ & $(0.0365)$ & $(0.0411)$ & (0) & (0) & - \\
\hline \multirow[t]{2}{*}{$(300,0.9,100)$} & 0.0719 & 0.1308 & 0.1236 & 0.0793 & 0.1270 & 0.1036 & 0 & 2 & 1 \\
\hline & $(0.0549)$ & $(0.0460)$ & $(0.0477)$ & $(0.0522)$ & $(0.0429)$ & $(0.0571)$ & (0) & (0) & - \\
\hline \multirow[t]{2}{*}{$(300,0.9,300)$} & 0.0778 & 0.1298 & 0.1199 & 0.0849 & 0.1251 & 0.1022 & 0 & 2 & 1 \\
\hline & $(0.0543)$ & $(0.0474)$ & $(0.0475)$ & $(0.0612)$ & $(0.0424)$ & $(0.0525)$ & (0) & (0) & - \\
\hline \multirow[t]{2}{*}{$(300,0.9,600)$} & 0.0764 & 0.1308 & 0.1239 & 0.0793 & 0.1246 & 0.1031 & 0 & 2 & 1 \\
\hline & $(0.0570)$ & $(0.0493)$ & $(0.0401)$ & $(0.0637)$ & $(0.0470)$ & $(0.0521)$ & (0) & (0) & - \\
\hline
\end{tabular}


Table 3.3: Simultaion results for $\kappa=0$ when $\tau=0.5$ and the error $\epsilon_{i}$ follows a Student's t distribution with scale parameter $1 / 3$ and degrees of freedom 3. Standard deviations are shown in the parentheses.

\begin{tabular}{|c|c|c|c|c|c|c|c|c|c|c|}
\hline \multirow[b]{2}{*}{ Method } & \multirow[b]{2}{*}{$\left(n, p_{n}\right)$} & \multicolumn{6}{|c|}{$\sqrt{\mathrm{AISE}}$} & \multirow[b]{2}{*}{ FD } & \multirow[b]{2}{*}{ TD } & \multirow[b]{2}{*}{$\mathrm{TM}$} \\
\hline & & $\alpha_{4,0}$ & $\alpha_{4,1}$ & $\alpha_{4,2}$ & $\alpha_{15,0}$ & $\alpha_{15,1}$ & $\alpha_{15,2}$ & & & \\
\hline \multirow[t]{18}{*}{ PQR } & $(100,100)$ & 0.0563 & 0.0920 & 0.0872 & 0.0897 & 0.0853 & 0.0644 & 0 & 2 & 1 \\
\hline & & $(0.0438)$ & $(0.0284)$ & $(0.0319)$ & $(0.0652)$ & $(0.0291)$ & $(0.0398)$ & (0) & (0) & - \\
\hline & $(100,300)$ & 0.0577 & 0.0898 & 0.0866 & 0.0881 & 0.0872 & 0.0679 & 0.005 & 2 & 0.995 \\
\hline & & $(0.0466)$ & $(0.0281)$ & $(0.0305)$ & $(0.0654)$ & $(0.0307)$ & $(0.0352)$ & $(0.070)$ & (0) & - \\
\hline & $(100,600)$ & 0.0660 & 0.0914 & 0.0886 & 0.0823 & 0.0866 & 0.0651 & 0.005 & 2 & 0.995 \\
\hline & & $(0.0481)$ & $(0.0304)$ & $(0.0299)$ & $(0.0667)$ & $(0.0315)$ & $(0.0372)$ & $(0.070)$ & (0) & - \\
\hline & $(200,100)$ & 0.0395 & 0.0787 & 0.0718 & 0.0593 & 0.0767 & 0.0420 & 0 & 2 & 1 \\
\hline & & $(0.0287)$ & $(0.0184)$ & $(0.0154)$ & $(0.0497)$ & $(0.0166)$ & $(0.0203)$ & (0) & (0) & - \\
\hline & $(200,300)$ & 0.0468 & 0.0765 & 0.0710 & 0.0631 & 0.0732 & 0.0421 & 0 & 2 & 1 \\
\hline & & $(0.0336)$ & $(0.0199)$ & $(0.0167)$ & $(0.0468)$ & $(0.0146)$ & $(0.0209)$ & (0) & (0) & - \\
\hline & $(200,600)$ & 0.0464 & 0.0785 & 0.0720 & 0.0557 & 0.0740 & 0.0443 & 0.005 & 2 & 0.995 \\
\hline & & $(0.0327)$ & $(0.0160)$ & $(0.0169)$ & $(0.0420)$ & $(0.0147)$ & $(0.0204)$ & $(0.070)$ & $(0)$ & - \\
\hline & $(300,100)$ & 0.0365 & 0.0721 & 0.0655 & 0.0451 & 0.0692 & 0.0342 & 0 & 2 & 1 \\
\hline & & $(0.0254)$ & $(0.0120)$ & $(0.0113)$ & $(0.0326)$ & $(0.0100)$ & $(0.0152)$ & (0) & $(0)$ & - \\
\hline & $(300,300)$ & 0.0392 & 0.0717 & 0.0682 & 0.0491 & 0.0708 & 0.0342 & 0 & 2 & 1 \\
\hline & & $(0.0296)$ & $(0.0131)$ & $(0.0132)$ & $(0.0369)$ & $(0.0133)$ & $(0.0177)$ & $(0)$ & $(0)$ & - \\
\hline & $(300,600)$ & 0.0393 & 0.0739 & 0.0662 & 0.0484 & 0.0700 & 0.0345 & 0 & 2 & 1 \\
\hline & & $(0.0289)$ & $(0.0119)$ & $(0.0123)$ & $(0.0382)$ & $(0.0119)$ & $(0.0156)$ & (0) & (0) & - \\
\hline \multirow[t]{18}{*}{ PLS } & $(100,100)$ & 0.0629 & 0.1032 & 0.0996 & 0.0943 & 0.0989 & 0.0730 & 0 & 2 & 1 \\
\hline & & $(0.0467)$ & $(0.0365)$ & $(0.0381)$ & $(0.0726)$ & $(0.0416)$ & $(0.0445)$ & $(0)$ & $(0)$ & - \\
\hline & $(100,300)$ & 0.0617 & 0.1012 & 0.0952 & 0.0894 & 0.0984 & 0.0707 & 0.015 & 2 & 0.995 \\
\hline & & $(0.0509)$ & $(0.0400)$ & $(0.0362)$ & $(0.0693)$ & $(0.0380)$ & $(0.0395)$ & $(0.212)$ & (0) & - \\
\hline & $(100,600)$ & 0.0697 & 0.1056 & 0.1005 & 0.0862 & 0.0994 & 0.0757 & 0.015 & 2 & 0.995 \\
\hline & & $(0.0506)$ & $(0.0554)$ & $(0.0442)$ & $(0.0673)$ & $(0.0386)$ & $(0.0479)$ & $(0.212)$ & (0) & - \\
\hline & $(200,100)$ & 0.0446 & 0.0875 & 0.0786 & 0.0624 & 0.0853 & 0.0541 & 0.010 & 2 & 0.995 \\
\hline & & $(0.0332)$ & $(0.0254)$ & $(0.0217)$ & $(0.0481)$ & $(0.0295)$ & $(0.0333)$ & $(0.141)$ & (0) & - \\
\hline & $(200,300)$ & 0.0518 & 0.0853 & 0.0791 & 0.0667 & 0.0810 & 0.0514 & 0 & 2 & 1 \\
\hline & & $(0.0393)$ & $(0.0214)$ & $(0.0210)$ & $(0.0467)$ & $(0.0225)$ & $(0.0329)$ & (0) & (0) & - \\
\hline & $(200,600)$ & 0.0501 & 0.0875 & 0.0814 & 0.0591 & 0.0836 & 0.0524 & 0 & 2 & 1 \\
\hline & & $(0.0390)$ & $(0.0238)$ & $(0.0303)$ & $(0.0469)$ & $(0.0220)$ & $(0.0306)$ & (0) & (0) & - \\
\hline & $(300,100)$ & 0.0408 & 0.0787 & 0.0737 & 0.0482 & 0.0756 & 0.0444 & 0 & 2 & 1 \\
\hline & & $(0.0294)$ & $(0.0151)$ & $(0.0181)$ & $(0.0355)$ & $(0.0153)$ & $(0.0232)$ & (0) & (0) & - \\
\hline & $(300,300)$ & 0.0456 & 0.0784 & 0.0741 & 0.0509 & 0.0784 & 0.0425 & 0 & 2 & 1 \\
\hline & & $(0.0336)$ & $(0.0152)$ & $(0.0163)$ & $(0.0393)$ & $(0.0164)$ & $(0.0214)$ & (0) & (0) & - \\
\hline & $(300,600)$ & 0.0439 & 0.0808 & 0.0749 & 0.0540 & 0.0761 & 0.0434 & 0 & 2 & 1 \\
\hline & & $(0.0312)$ & $(0.0154)$ & $(0.0158)$ & $(0.0399)$ & $(0.0153)$ & $(0.0224)$ & (0) & (0) & - \\
\hline
\end{tabular}


Table 3.4: Simulation results for $\kappa=0$ when $\tau=0.7,0.9$ and the error $\epsilon_{i}$ follows a Student's t distribution with scale parameter $1 / 3$ and degrees of freedom 3. Standard deviations are shown in the parentheses.

\begin{tabular}{|c|c|c|c|c|c|c|c|c|c|}
\hline \multirow[b]{2}{*}{$\left(n, \tau, p_{n}\right)$} & \multicolumn{6}{|c|}{$\sqrt{\mathrm{AISE}}$} & \multirow[b]{2}{*}{ FD } & \multirow[b]{2}{*}{$\mathrm{TD}$} & \multirow[b]{2}{*}{$\mathrm{TM}$} \\
\hline & $\alpha_{4,0}$ & $\alpha_{4,1}$ & $\alpha_{4,2}$ & $\alpha_{15,0}$ & $\alpha_{15,1}$ & $\alpha_{15,2}$ & & & \\
\hline \multirow{2}{*}{$(100,0.7,100)$} & 0.0623 & 0.1011 & 0.0926 & 0.0904 & 0.0947 & 0.0677 & 0.005 & 2 & 0.995 \\
\hline & $(0.0452)$ & $(0.0355)$ & $(0.0310)$ & $(0.0617)$ & $(0.0348)$ & $(0.0384)$ & $(0.070)$ & (0) & - \\
\hline \multirow[t]{2}{*}{$(100,0.7,300)$} & 0.0644 & 0.0986 & 0.0929 & 0.0869 & 0.0927 & 0.0733 & 0.030 & 2 & 0.970 \\
\hline & $(0.0472)$ & $(0.0332)$ & $(0.0346)$ & $(0.0672)$ & $(0.0320)$ & $(0.0392)$ & $(0.171)$ & (0) & - \\
\hline \multirow[t]{2}{*}{$(100,0.7,600)$} & 0.0697 & 0.0981 & 0.0930 & 0.0841 & 0.0943 & 0.0726 & 0.015 & 2 & 0.985 \\
\hline & $(0.0477)$ & $(0.0347)$ & $(0.0380)$ & $(0.0677)$ & $(0.0321)$ & $(0.0408)$ & $(0.121)$ & (0) & - \\
\hline \multirow[t]{2}{*}{$(100,0.9,100)$} & 0.0733 & 0.1275 & 0.1179 & 0.0918 & 0.1204 & 0.0901 & 0.010 & 2 & 0.990 \\
\hline & $(0.0539)$ & $(0.0555)$ & $(0.0494)$ & $(0.0690)$ & $(0.0551)$ & $(0.0490)$ & $(0.099)$ & (0) & - \\
\hline \multirow[t]{2}{*}{$(100,0.9,300)$} & 0.0808 & 0.1234 & 0.1176 & 0.0981 & 0.1162 & 0.0969 & 0.005 & 2 & 0.995 \\
\hline & $(0.0573)$ & $(0.0427)$ & $(0.0501)$ & $(0.0836)$ & $(0.0435)$ & $(0.0519)$ & $(0.070)$ & (0) & - \\
\hline \multirow[t]{2}{*}{$(100,0.9,600)$} & 0.0820 & 0.1216 & 0.1179 & 0.0896 & 0.1196 & 0.0989 & 0 & 2 & 1 \\
\hline & $(0.0583)$ & $(0.0464)$ & $(0.0444)$ & $(0.0719)$ & $(0.0479)$ & $(0.0567)$ & $(0)$ & $(0)$ & - \\
\hline \multirow[t]{2}{*}{$(200,0.7,100)$} & 0.0447 & 0.0811 & 0.0740 & 0.0580 & 0.0765 & 0.0455 & 0 & 2 & 1 \\
\hline & $(0.0310)$ & $(0.0191)$ & $(0.0179)$ & $(0.0439)$ & $(0.0168)$ & $(0.0229)$ & (0) & (0) & - \\
\hline \multirow[t]{2}{*}{$(200,0.7,300)$} & 0.0501 & 0.0805 & 0.0737 & 0.0632 & 0.0769 & 0.0461 & 0 & 2 & 1 \\
\hline & $(0.0346)$ & $(0.0207)$ & $(0.0182)$ & $(0.0455)$ & $(0.0182)$ & $(0.0236)$ & (0) & (0) & - \\
\hline \multirow[t]{2}{*}{$(200,0.7,600)$} & 0.0469 & 0.0827 & 0.0772 & 0.0588 & 0.0780 & 0.0479 & 0 & 2 & 1 \\
\hline & $(0.0338)$ & $(0.0186)$ & $(0.0210)$ & $(0.0460)$ & $(0.0165)$ & $(0.0244)$ & (0) & (0) & - \\
\hline \multirow[t]{2}{*}{$(200,0.9,100)$} & 0.0498 & 0.0983 & 0.0914 & 0.0685 & 0.0952 & 0.0628 & 0 & 2 & 1 \\
\hline & $(0.0402)$ & $(0.0251)$ & $(0.0278)$ & $(0.0530)$ & $(0.0279)$ & $(0.0310)$ & (0) & (0) & - \\
\hline \multirow[t]{2}{*}{$(200,0.9,300)$} & 0.0593 & 0.0990 & 0.0931 & 0.0683 & 0.0910 & 0.0664 & 0 & 2 & 1 \\
\hline & $(0.0438)$ & $(0.0277)$ & $(0.0303)$ & $(0.0514)$ & $(0.0236)$ & $(0.0332)$ & (0) & (0) & - \\
\hline \multirow[t]{2}{*}{$(200,0.9,600)$} & 0.0564 & 0.0993 & 0.0934 & 0.0700 & 0.0943 & 0.0628 & 0 & 2 & 1 \\
\hline & $(0.0391)$ & $(0.0292)$ & $(0.0325)$ & $(0.0524)$ & $(0.0255)$ & $(0.0357)$ & $(0)$ & $(0)$ & - \\
\hline \multirow[t]{2}{*}{$(300,0.7,100)$} & 0.0391 & 0.0763 & 0.0691 & 0.0461 & 0.0724 & 0.0378 & 0 & 2 & 1 \\
\hline & $(0.0273)$ & $(0.0118)$ & $(0.0126)$ & $(0.0335)$ & $(0.0110)$ & $(0.0166)$ & (0) & (0) & - \\
\hline \multirow[t]{2}{*}{$(300,0.7,300)$} & 0.0396 & 0.0757 & 0.0715 & 0.0492 & 0.0739 & 0.0378 & 0 & 2 & 1 \\
\hline & $(0.0311)$ & $(0.0130)$ & $(0.0151)$ & $(0.0374)$ & $(0.0134)$ & $(0.0202)$ & (0) & (0) & - \\
\hline \multirow[t]{2}{*}{$(300,0.7,600)$} & 0.0414 & 0.0776 & 0.0699 & 0.0511 & 0.0734 & 0.0381 & 0 & 2 & 1 \\
\hline & $(0.0295)$ & $(0.0142)$ & $(0.0130)$ & $(0.0386)$ & $(0.0131)$ & $(0.0182)$ & (0) & (0) & - \\
\hline \multirow[t]{2}{*}{$(300,0.9,100)$} & 0.0452 & 0.0894 & 0.0819 & 0.0523 & 0.0861 & 0.0543 & 0 & 2 & 1 \\
\hline & $(0.0349)$ & $(0.0184)$ & $(0.0207)$ & $(0.0384)$ & $(0.0182)$ & $(0.0286)$ & (0) & (0) & - \\
\hline \multirow[t]{2}{*}{$(300,0.9,300)$} & 0.0508 & 0.0897 & 0.0833 & 0.0535 & 0.0848 & 0.0508 & 0 & 2 & 1 \\
\hline & $(0.0390)$ & $(0.0204)$ & $(0.0203)$ & $(0.0386)$ & $(0.0209)$ & $(0.0255)$ & (0) & (0) & - \\
\hline \multirow[t]{2}{*}{$(300,0.9,600)$} & 0.0479 & 0.0908 & 0.0844 & 0.0564 & 0.0855 & 0.0524 & 0 & 2 & 1 \\
\hline & $(0.0336)$ & $(0.0223)$ & $(0.0225)$ & $(0.0421)$ & $(0.0196)$ & $(0.0281)$ & (0) & (0) & - \\
\hline
\end{tabular}



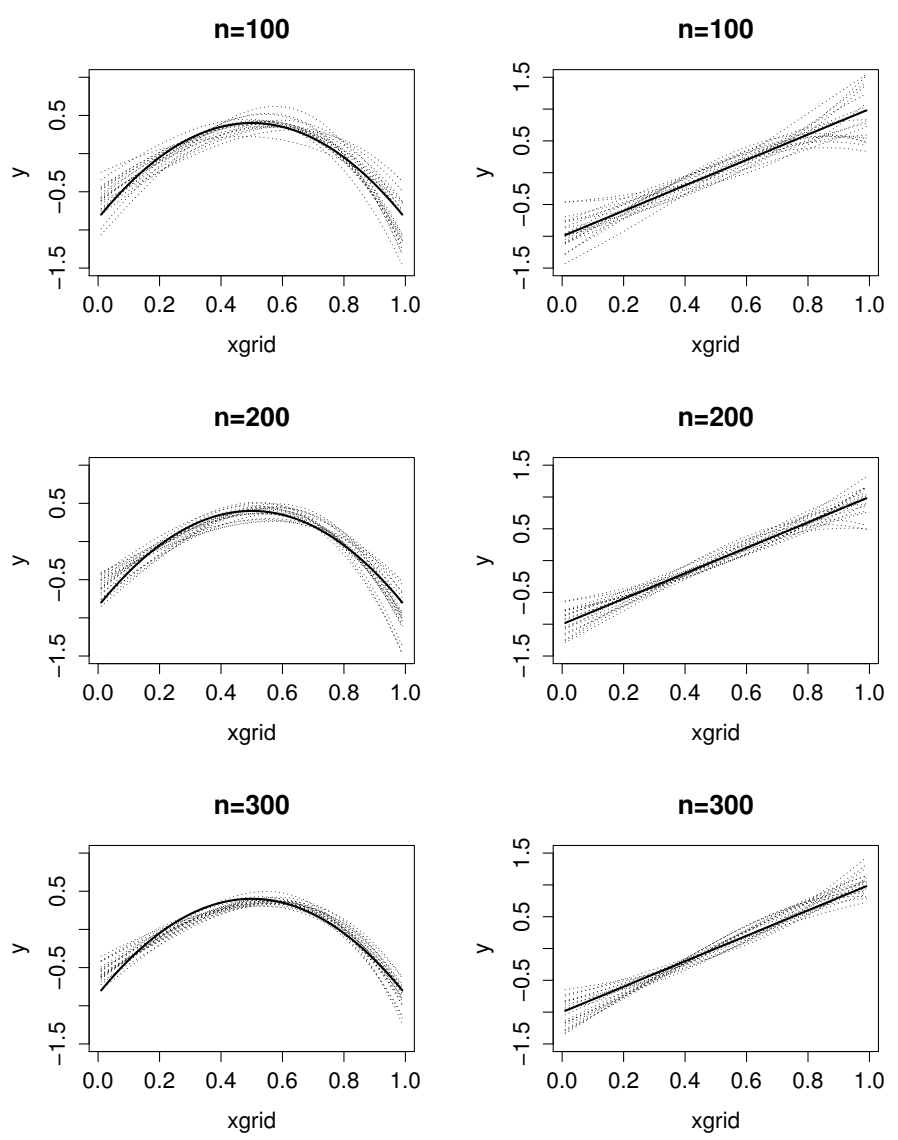

Figure 3.1: The SCAD penalized quantile estimates for $\kappa=0$ with standard normal error. Left: Estimates for $\alpha_{4,1}(\cdot)$ when $\tau=0.5, p_{n}=300$; Right: Estimates for $\alpha_{15,2}(\cdot)$ when $\tau=0.9, p_{n}=100$. The solid curve is the true function and the dotted curves are the estimates for 20 randomly chosen replicates. 

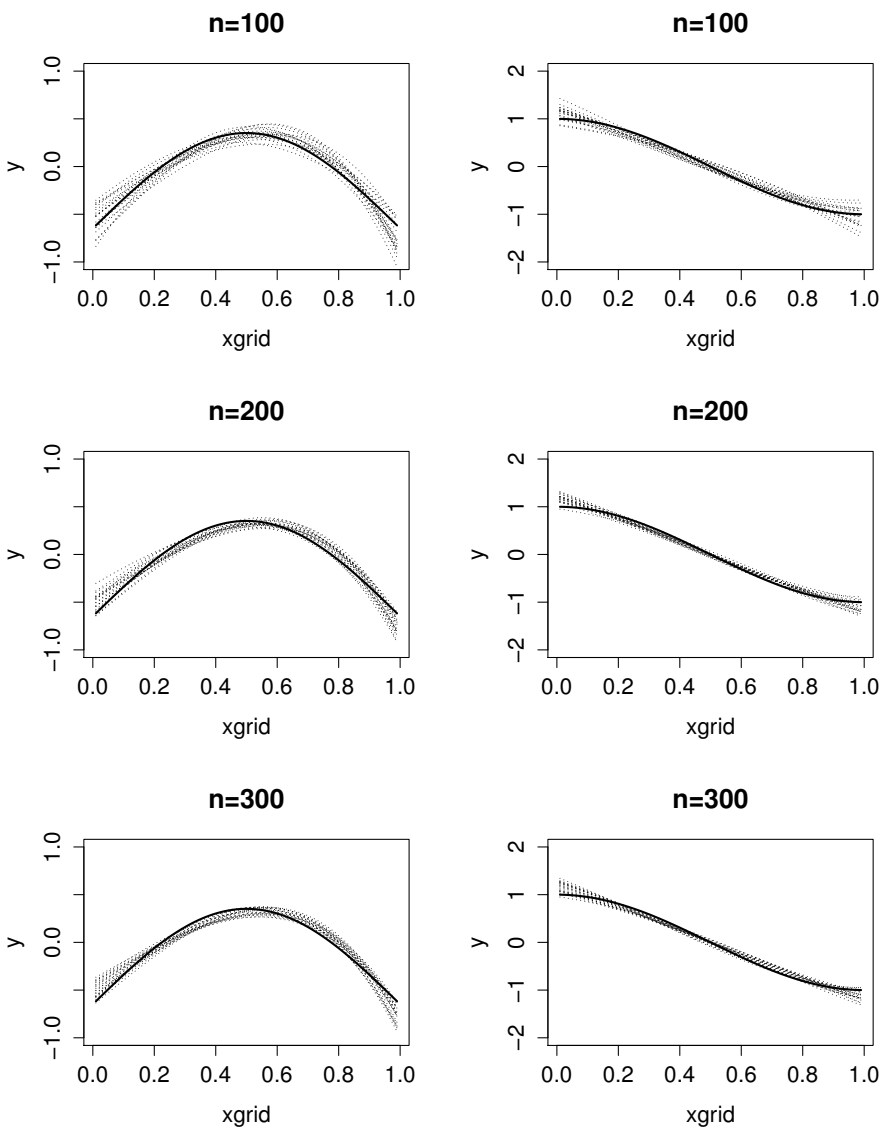

Figure 3.2: The SCAD penalized quantile estimates for $\kappa=0$ when the error follows a Student's t distribution with degrees of freedom 3 and scale parameter $1 / 3$. Left: Estimates for $\alpha_{4,2}(\cdot)$ when $\tau=0.5, p_{n}=600$; Right: Estimates for $\alpha_{15,1}(\cdot)$ when $\tau=0.7, p_{n}=300$. The solid curve is the true function and the dotted curves are the estimates for 20 randomly chosen replicates. 
Table 3.5: Simultaion results for $\kappa=1$ when $\epsilon_{i}$ follows a standard normal distribution and $\tau=0.5$. Standard deviations are shown in the parentheses.

\begin{tabular}{|c|c|c|c|c|c|c|c|c|c|c|}
\hline \multirow[b]{2}{*}{ Method } & \multirow[b]{2}{*}{$\left(n, p_{n}\right)$} & \multicolumn{6}{|c|}{$\sqrt{\mathrm{AISE}}$} & \multirow[b]{2}{*}{ FD } & \multirow[b]{2}{*}{ TD } & \multirow[b]{2}{*}{$\mathrm{TM}$} \\
\hline & & $\alpha_{4,0}$ & $\alpha_{4,1}$ & $\alpha_{4,2}$ & $\alpha_{15,0}$ & $\alpha_{15,1}$ & $\alpha_{15,2}$ & & & \\
\hline \multirow[t]{18}{*}{ PQR } & $(100,100)$ & 0.2194 & 0.3735 & 0.3587 & 0.1917 & 0.3156 & 0.3242 & 0 & 2 & 1 \\
\hline & & $(0.1617)$ & $(0.2058)$ & $(0.1870)$ & $(0.1529)$ & $(0.1786)$ & $(0.1866)$ & (0) & (0) & - \\
\hline & $(100,300)$ & 0.2302 & 0.3541 & 0.3920 & 0.2101 & 0.2930 & 0.3103 & 0 & 2 & 1 \\
\hline & & $(0.1860)$ & $(0.1693)$ & $(0.2203)$ & $(0.1650)$ & $(0.1445)$ & $(0.1751)$ & (0) & (0) & - \\
\hline & $(100,600)$ & 0.2663 & 0.3638 & 0.3674 & 0.1961 & 0.3240 & 0.3138 & 0 & 1.985 & 0.985 \\
\hline & & $(0.3346)$ & $(0.1808)$ & $(0.1914)$ & $(0.1517)$ & $(0.1549)$ & $(0.1817)$ & (0) & $(0.121)$ & - \\
\hline & $(200,100)$ & 0.1709 & 0.2479 & 0.2631 & 0.1405 & 0.2143 & 0.2027 & 0 & 2 & 1 \\
\hline & & $(0.1319)$ & $(0.1162)$ & $(0.1376)$ & $(0.1063)$ & $(0.0951)$ & $(0.1082)$ & (0) & (0) & - \\
\hline & $(200,300)$ & 0.1696 & 0.2383 & 0.2704 & 0.1449 & 0.2135 & 0.2034 & 0 & 2 & 1 \\
\hline & & $(0.1287)$ & $(0.1173)$ & $(0.1331)$ & $(0.1049)$ & $(0.1012)$ & $(0.1026)$ & (0) & (0) & - \\
\hline & $(200,600)$ & 0.1615 & 0.2636 & 0.2604 & 0.1579 & 0.2127 & 0.2080 & 0 & 2 & 1 \\
\hline & & $(0.1170)$ & $(0.1192)$ & $(0.1324)$ & $(0.1080)$ & $(0.0925)$ & $(0.1200)$ & (0) & (0) & - \\
\hline & $(300,100)$ & 0.1424 & 0.2167 & 0.2135 & 0.1124 & 0.1742 & 0.1608 & 0 & 2 & 1 \\
\hline & & $(0.0966)$ & $(0.0942)$ & $(0.1002)$ & $(0.0824)$ & $(0.0797)$ & $(0.0796)$ & (0) & (0) & - \\
\hline & $(300,300)$ & 0.1463 & 0.2182 & 0.2102 & 0.1209 & 0.1759 & 0.1636 & 0 & 1.995 & 0.995 \\
\hline & & $(0.1929)$ & $(0.0978)$ & $(0.0906)$ & $(0.0942)$ & $(0.0751)$ & $(0.0825)$ & (0) & $(0.070)$ & - \\
\hline & $(300,600)$ & 0.1477 & 0.2166 & 0.2141 & 0.1104 & 0.1769 & 0.1617 & 0 & 1.995 & 0.995 \\
\hline & & $(0.1934)$ & $(0.1053)$ & $(0.0947)$ & $(0.0851)$ & $(0.0765)$ & $(0.0851)$ & $(0)$ & $(0.070)$ & - \\
\hline \multirow[t]{18}{*}{ PLS } & $(100,100)$ & 0.1943 & 0.3816 & 0.3446 & 0.1989 & 0.3243 & 0.3301 & 0.010 & 2 & 0.990 \\
\hline & & $(0.1654)$ & $(0.1730)$ & $(0.1593)$ & $(0.1487)$ & $(0.1660)$ & $(0.1404)$ & $(0.099)$ & (0) & - \\
\hline & $(100,300)$ & 0.2073 & 0.3619 & 0.3992 & 0.1935 & 0.3190 & 0.3253 & 0.015 & 2 & 0.985 \\
\hline & & $(0.1802)$ & $(0.1739)$ & $(0.1987)$ & $(0.1537)$ & $(0.1424)$ & $(0.1727)$ & $(0.121)$ & (0) & - \\
\hline & $(100,600)$ & 0.2092 & 0.3727 & 0.3638 & 0.1939 & 0.3297 & 0.3152 & 0.015 & 2 & 0.990 \\
\hline & & $(0.1716)$ & $(0.1717)$ & $(0.1745)$ & $(0.1361)$ & $(0.1566)$ & $(0.1619)$ & $(0.157)$ & (0) & - \\
\hline & $(200,100)$ & 0.1558 & 0.2369 & 0.2506 & 0.1335 & 0.2151 & 0.2149 & 0 & 2 & 1 \\
\hline & & $(0.1188)$ & $(0.1127)$ & $(0.1191)$ & $(0.1019)$ & $(0.0994)$ & $(0.1015)$ & (0) & (0) & - \\
\hline & $(200,300)$ & 0.1490 & 0.2441 & 0.2565 & 0.1401 & 0.2146 & 0.2119 & 0 & 2 & 1 \\
\hline & & $(0.1153)$ & $(0.1062)$ & $(0.1226)$ & $(0.1046)$ & $(0.0985)$ & $(0.1019)$ & (0) & (0) & - \\
\hline & $(200,600)$ & 0.1449 & 0.2593 & 0.2571 & 0.1365 & 0.2083 & 0.2150 & 0 & 2 & 1 \\
\hline & & $(0.1101)$ & $(0.1224)$ & $(0.1262)$ & $(0.1110)$ & $(0.0929)$ & $(0.1111)$ & (0) & (0) & - \\
\hline & $(300,100)$ & 0.1184 & 0.2069 & 0.2014 & 0.0990 & 0.1722 & 0.1678 & 0 & 2 & 1 \\
\hline & & $(0.0923)$ & $(0.0924)$ & $(0.0915)$ & $(0.0757)$ & $(0.0772)$ & $(0.0796)$ & (0) & (0) & - \\
\hline & $(300,300)$ & 0.1265 & 0.1995 & 0.2001 & 0.1102 & 0.1784 & 0.1596 & 0 & 2 & 1 \\
\hline & & $(0.0824)$ & $(0.0986)$ & $(0.0966)$ & $(0.0859)$ & $(0.0734)$ & $(0.0762)$ & (0) & (0) & - \\
\hline & $(300,600)$ & 0.1177 & 0.2041 & 0.2048 & 0.1055 & 0.1724 & 0.1662 & 0 & 2 & 1 \\
\hline & & $(0.0969)$ & $(0.0934)$ & $(0.0966)$ & $(0.0800)$ & $(0.0821)$ & $(0.0786)$ & (0) & (0) & - \\
\hline
\end{tabular}


Table 3.6: Simulation results for $\kappa=1$ when $\epsilon_{i}$ follows a standard normal distribution and $\tau=0.7,0.9$. Standard deviations are shown in the parentheses.

\begin{tabular}{|c|c|c|c|c|c|c|c|c|c|}
\hline \multirow[b]{2}{*}{$\left(n, \tau, p_{n}\right)$} & \multicolumn{6}{|c|}{$\sqrt{\mathrm{AISE}}$} & \multirow[b]{2}{*}{ FD } & \multirow[b]{2}{*}{ TD } & \multirow[b]{2}{*}{$\mathrm{TM}$} \\
\hline & $\alpha_{4,0}$ & $\alpha_{4,1}$ & $\alpha_{4,2}$ & $\alpha_{15,0}$ & $\alpha_{15,1}$ & $\alpha_{15,2}$ & & & \\
\hline \multirow{2}{*}{$(100,0.7,100)$} & 0.2430 & 0.3838 & 0.3782 & 0.2098 & 0.3235 & 0.3211 & 0.005 & 2 & 0.995 \\
\hline & $(0.2004)$ & $(0.2044)$ & $(0.1926)$ & $(0.1638)$ & $(0.1546)$ & $(0.1825)$ & $(0.070)$ & (0) & - \\
\hline \multirow[t]{2}{*}{$(100,0.7,300)$} & 0.2409 & 0.3777 & 0.4203 & 0.2173 & 0.3023 & 0.3173 & 0 & 2 & 1 \\
\hline & $(0.2111)$ & $(0.1790)$ & $(0.2288)$ & $(0.1592)$ & $(0.1680)$ & $(0.1787)$ & $(0)$ & (0) & - \\
\hline \multirow[t]{2}{*}{$(100,0.7,600)$} & 0.2556 & 0.3780 & 0.3851 & 0.2151 & 0.3140 & 0.3089 & 0 & 2 & 1 \\
\hline & $(0.1820)$ & $(0.1843)$ & $(0.1813)$ & $(0.1492)$ & $(0.1584)$ & $(0.1782)$ & $(0)$ & (0) & - \\
\hline \multirow[t]{2}{*}{$(100,0.9,100)$} & 0.2916 & 0.4233 & 0.4173 & 0.2349 & 0.3803 & 0.3744 & 0 & 2 & 1 \\
\hline & $(0.2212)$ & $(0.2362)$ & $(0.2090)$ & $(0.2028)$ & $(0.2152)$ & $(0.1948)$ & (0) & (0) & - \\
\hline \multirow[t]{2}{*}{$(100,0.9,300)$} & 0.2984 & 0.4488 & 0.4733 & 0.2517 & 0.3791 & 0.3628 & 0 & 2 & 1 \\
\hline & $(0.2245)$ & $(0.2254)$ & $(0.2538)$ & $(0.1906)$ & $(0.1973)$ & $(0.1876)$ & $(0)$ & (0) & - \\
\hline \multirow[t]{2}{*}{$(100,0.9,600)$} & 0.3028 & 0.4234 & 0.4384 & 0.2618 & 0.3657 & 0.3428 & 0 & 2 & 1 \\
\hline & $(0.2233)$ & $(0.2036)$ & $(0.2163)$ & $(0.1976)$ & $(0.1890)$ & $(0.1960)$ & $(0)$ & $(0)$ & - \\
\hline \multirow[t]{2}{*}{$(200,0.7,100)$} & 0.1700 & 0.2467 & 0.2696 & 0.1439 & 0.2135 & 0.2168 & 0 & 2 & 1 \\
\hline & $(0.1329)$ & $(0.1232)$ & $(0.1396)$ & $(0.1023)$ & $(0.1091)$ & $(0.1112)$ & (0) & (0) & - \\
\hline \multirow[t]{2}{*}{$(200,0.7,300)$} & 0.1874 & 0.2530 & 0.2682 & 0.1606 & 0.2229 & 0.2102 & 0 & 2 & 1 \\
\hline & $(0.1452)$ & $(0.1212)$ & $(0.1352)$ & $(0.1262)$ & $(0.1059)$ & $(0.1099)$ & (0) & (0) & - \\
\hline \multirow[t]{2}{*}{$(200,0.7,600)$} & 0.1660 & 0.2717 & 0.2633 & 0.1503 & 0.2171 & 0.2137 & 0 & 2 & 1 \\
\hline & $(0.1247)$ & $(0.1307)$ & $(0.1280)$ & $(0.1142)$ & $(0.1022)$ & $(0.1194)$ & (0) & (0) & - \\
\hline \multirow[t]{2}{*}{$(200,0.9,100)$} & 0.2098 & 0.2922 & 0.3302 & 0.1774 & 0.2578 & 0.2533 & 0 & 2 & 1 \\
\hline & $(0.1657)$ & $(0.1490)$ & $(0.1628)$ & $(0.1295)$ & $(0.1229)$ & $(0.1257)$ & 0 & 2 & 1 \\
\hline \multirow[t]{2}{*}{$(200,0.9,300)$} & 0.2011 & 0.2955 & 0.3161 & 0.1893 & 0.2607 & 0.2529 & 0 & 2 & 1 \\
\hline & $(0.1612)$ & $(0.1530)$ & $(0.1582)$ & $(0.1408)$ & $(0.1273)$ & $(0.1426)$ & (0) & (0) & - \\
\hline \multirow[t]{2}{*}{$(200,0.9,600)$} & 0.2217 & 0.3227 & 0.3175 & 0.1799 & 0.2717 & 0.2443 & 0 & 2 & 1 \\
\hline & $(0.1526)$ & $(0.1571)$ & $(0.1532)$ & $(0.1342)$ & $(0.1337)$ & $(0.1424)$ & $(0)$ & $(0)$ & - \\
\hline \multirow[t]{2}{*}{$(300,0.7,100)$} & 0.1297 & 0.2201 & 0.2207 & 0.1094 & 0.1802 & 0.1783 & 0 & 2 & 1 \\
\hline & $(0.1098)$ & $(0.1077)$ & $(0.1120)$ & $(0.0807)$ & $(0.0823)$ & $(0.0970)$ & (0) & (0) & - \\
\hline \multirow[t]{2}{*}{$(300,0.7,300)$} & 0.1376 & 0.2188 & 0.2124 & 0.1250 & 0.1786 & 0.1632 & 0 & 2 & 1 \\
\hline & $(0.0983)$ & $(0.1069)$ & $(0.1056)$ & $(0.0862)$ & $(0.0826)$ & $(0.0828)$ & (0) & (0) & - \\
\hline \multirow[t]{2}{*}{$(300,0.7,600)$} & 0.1417 & 0.2190 & 0.2230 & 0.1181 & 0.1848 & 0.1729 & 0 & 2 & 1 \\
\hline & $(0.1111)$ & $(0.0998)$ & $(0.1136)$ & $(0.0901)$ & $(0.0905)$ & $(0.0848)$ & (0) & (0) & - \\
\hline \multirow[t]{2}{*}{$(300,0.9,100)$} & 0.1752 & 0.2581 & 0.2519 & 0.1507 & 0.2228 & 0.2188 & 0 & 2 & 1 \\
\hline & $(0.1295)$ & $(0.1248)$ & $(0.1293)$ & $(0.1002)$ & $(0.1017)$ & $(0.1120)$ & (0) & $(0)$ & - \\
\hline \multirow[t]{2}{*}{$(300,0.9,300)$} & 0.1753 & 0.2553 & 0.2506 & 0.1441 & 0.2096 & 0.1999 & 0 & 2 & 1 \\
\hline & $(0.1295)$ & $(0.1307)$ & $(0.1179)$ & $(0.1043)$ & $(0.0947)$ & $(0.1038)$ & (0) & (0) & - \\
\hline \multirow[t]{2}{*}{$(300,0.9,600)$} & 0.1760 & 0.2707 & 0.2664 & 0.1465 & 0.2115 & 0.2006 & 0 & 2 & 1 \\
\hline & $(0.1419)$ & $(0.1332)$ & $(0.1188)$ & $(0.1042)$ & $(0.1050)$ & $(0.1149)$ & (0) & (0) & - \\
\hline
\end{tabular}


Table 3.7: Simultaion results for $\kappa=1$ when $\tau=0.5$ and the error $\epsilon_{i}$ follows a Student's t distribution with scale parameter $1 / 3$ and degrees of freedom 3. Standard deviations are shown in the parentheses.

\begin{tabular}{|c|c|c|c|c|c|c|c|c|c|c|}
\hline \multirow[b]{2}{*}{ Method } & \multirow[b]{2}{*}{$\left(n, p_{n}\right)$} & \multicolumn{6}{|c|}{$\sqrt{\mathrm{AISE}}$} & \multirow[b]{2}{*}{ FD } & \multirow[b]{2}{*}{ TD } & \multirow[b]{2}{*}{$\mathrm{TM}$} \\
\hline & & $\alpha_{4,0}$ & $\alpha_{4,1}$ & $\alpha_{4,2}$ & $\alpha_{15,0}$ & $\alpha_{15,1}$ & $\alpha_{15,2}$ & & & \\
\hline \multirow[t]{18}{*}{ PQR } & $(100,100)$ & 0.1021 & 0.1651 & 0.1563 & 0.1182 & 0.1402 & 0.1241 & 0 & 2 & 1 \\
\hline & & $(0.0771)$ & $(0.0732)$ & $(0.0707)$ & $(0.0840)$ & $(0.0610)$ & $(0.0699)$ & (0) & (0) & - \\
\hline & $(100,300)$ & 0.0975 & 0.1595 & 0.1619 & 0.1112 & 0.1426 & 0.1360 & 0 & 2 & 1 \\
\hline & & $(0.0793)$ & $(0.0657)$ & $(0.0734)$ & $(0.0860)$ & $(0.0623)$ & $(0.0834)$ & (0) & (0) & - \\
\hline & $(100,600)$ & 0.1091 & 0.1580 & 0.1667 & 0.1107 & 0.1360 & 0.1271 & 0 & 2 & 1 \\
\hline & & $(0.0807)$ & $(0.0743)$ & $(0.0723)$ & $(0.0871)$ & $(0.0615)$ & $(0.0735)$ & (0) & (0) & - \\
\hline & $(200,100)$ & 0.0639 & 0.1172 & 0.1126 & 0.0743 & 0.1058 & 0.0772 & 0 & 2 & 1 \\
\hline & & $(0.0468)$ & $(0.0414)$ & $(0.0436)$ & $(0.0622)$ & $(0.0358)$ & $(0.0406)$ & (0) & $(0)$ & - \\
\hline & $(200,300)$ & 0.0730 & 0.1179 & 0.1119 & 0.0776 & 0.1004 & 0.0795 & 0 & 2 & 1 \\
\hline & & $(0.0520)$ & $(0.0457)$ & $(0.0409)$ & $(0.0559)$ & $(0.0312)$ & $(0.0416)$ & (0) & (0) & - \\
\hline & $(200,600)$ & 0.0706 & 0.1195 & 0.1138 & 0.0700 & 0.1003 & 0.0851 & 0 & 2 & 1 \\
\hline & & $(0.0506)$ & $(0.0381)$ & $(0.0421)$ & $(0.0526)$ & $(0.0301)$ & $(0.0416)$ & (0) & $(0)$ & - \\
\hline & $(300,100)$ & 0.0560 & 0.1011 & 0.0966 & 0.0591 & 0.0913 & 0.0630 & 0 & 2 & 1 \\
\hline & & $(0.0409)$ & $(0.0286)$ & $(0.0280)$ & $(0.0418)$ & $(0.0210)$ & $(0.0302)$ & (0) & $(0)$ & - \\
\hline & $(300,300)$ & 0.0577 & 0.1012 & 0.1060 & 0.0627 & 0.0944 & 0.0629 & 0 & 2 & 1 \\
\hline & & $(0.0483)$ & $(0.0303)$ & $(0.0351)$ & $(0.0444)$ & $(0.0266)$ & $(0.0350)$ & (0) & $(0)$ & - \\
\hline & $(300,600)$ & 0.0614 & 0.1047 & 0.0968 & 0.0629 & 0.0912 & 0.0639 & 0 & 2 & 1 \\
\hline & & $(0.0456)$ & $(0.0288)$ & $(0.0314)$ & $(0.0474)$ & $(0.0257)$ & $(0.0321)$ & (0) & (0) & - \\
\hline \multirow[t]{18}{*}{ PLS } & $(100,100)$ & 0.1170 & 0.1963 & 0.2026 & 0.1337 & 0.1675 & 0.1563 & 0 & 2 & 1 \\
\hline & & $(0.0940)$ & $(0.1006)$ & $(0.0996)$ & $(0.1022)$ & $(0.0988)$ & $(0.0921)$ & (0) & $(0)$ & - \\
\hline & $(100,300)$ & 0.1162 & 0.1951 & 0.1844 & 0.1274 & 0.1665 & 0.1496 & 0.025 & 2 & 0.985 \\
\hline & & $(0.0902)$ & $(0.1512)$ & $(0.0996)$ & $(0.0959)$ & $(0.1028)$ & $(0.0802)$ & $(0.211)$ & (0) & - \\
\hline & $(100,600)$ & 0.1248 & 0.1993 & 0.2039 & 0.1203 & 0.1701 & 0.1635 & 0.015 & 2 & 0.995 \\
\hline & & $(0.0967)$ & $(0.1474)$ & $(0.1213)$ & $(0.1014)$ & $(0.0968)$ & $(0.1114)$ & $(0.212)$ & (0) & - \\
\hline & $(200,100)$ & 0.0804 & 0.1423 & 0.1337 & 0.0853 & 0.1298 & 0.1136 & 0.010 & 2 & 0.995 \\
\hline & & $(0.0664)$ & $(0.0769)$ & $(0.0666)$ & $(0.0711)$ & $(0.0762)$ & $(0.0733)$ & $(0.141)$ & (0) & - \\
\hline & $(200,300)$ & 0.0897 & 0.1418 & 0.1401 & 0.0861 & 0.1212 & 0.1052 & 0 & 2 & 1 \\
\hline & & $(0.0734)$ & $(0.0672)$ & $(0.0614)$ & $(0.0671)$ & $(0.0582)$ & $(0.0608)$ & (0) & (0) & - \\
\hline & $(200,600)$ & 0.0873 & 0.1460 & 0.1461 & 0.0776 & 0.1226 & 0.1110 & 0.020 & 2 & 0.990 \\
\hline & & $(0.0723)$ & $(0.0795)$ & $(0.0917)$ & $(0.0678)$ & $(0.0600)$ & $(0.0710)$ & $(0.223)$ & (0) & - \\
\hline & $(300,100)$ & 0.0716 & 0.1168 & 0.1219 & 0.0689 & 0.1042 & 0.0923 & 0 & 2 & 1 \\
\hline & & $(0.0564)$ & $(0.0520)$ & $(0.0565)$ & $(0.0498)$ & $(0.0410)$ & $(0.0505)$ & (0) & (0) & - \\
\hline & $(300,300)$ & 0.0752 & 0.1167 & 0.1217 & 0.0715 & 0.1098 & 0.0868 & 0 & 2 & 1 \\
\hline & & $(0.0630)$ & $(0.0494)$ & $(0.0530)$ & $(0.0526)$ & $(0.0415)$ & $(0.0483)$ & (0) & (0) & - \\
\hline & $(300,600)$ & 0.0750 & 0.1229 & 0.1207 & 0.0769 & 0.1043 & 0.0884 & 0 & 2 & 1 \\
\hline & & $(0.0565)$ & $(0.0503)$ & $(0.0482)$ & $(0.0564)$ & $(0.0408)$ & $(0.0501)$ & (0) & (0) & - \\
\hline
\end{tabular}


Table 3.8: Simulation results for $\kappa=1$ when $\tau=0.7,0.9$ and the error $\epsilon_{i}$ follows a Student's t distribution with scale parameter $1 / 3$ and degrees of freedom 3. Standard deviations are shown in the parentheses.

\begin{tabular}{|c|c|c|c|c|c|c|c|c|c|}
\hline \multirow[b]{2}{*}{$\left(n, \tau, p_{n}\right)$} & \multicolumn{6}{|c|}{$\sqrt{\mathrm{AISE}}$} & \multirow[b]{2}{*}{ FD } & \multirow[b]{2}{*}{ TD } & \multirow[b]{2}{*}{$\mathrm{TM}$} \\
\hline & $\alpha_{4,0}$ & $\alpha_{4,1}$ & $\alpha_{4,2}$ & $\alpha_{15,0}$ & $\alpha_{15,1}$ & $\alpha_{15,2}$ & & & \\
\hline \multirow{2}{*}{$(100,0.7,100)$} & 0.1072 & 0.1697 & 0.1696 & 0.1148 & 0.1459 & 0.1386 & 0 & 2 & 1 \\
\hline & $(0.0862)$ & $(0.0787)$ & $(0.0820)$ & $(0.0886)$ & $(0.0629)$ & $(0.0813)$ & $(0)$ & (0) & - \\
\hline \multirow[t]{2}{*}{$(100,0.7,300)$} & 0.1043 & 0.1755 & 0.1755 & 0.1170 & 0.1496 & 0.1420 & 0 & 2 & 1 \\
\hline & $(0.0798)$ & $(0.0845)$ & $(0.0854)$ & $(0.0885)$ & $(0.0643)$ & $(0.0766)$ & $(0)$ & (0) & - \\
\hline \multirow[t]{2}{*}{$(100,0.7,600)$} & 0.1119 & 0.1709 & 0.1729 & 0.1085 & 0.1490 & 0.1410 & 0 & 2 & 1 \\
\hline & $(0.0854)$ & $(0.0834)$ & $(0.0801)$ & $(0.0935)$ & $(0.0758)$ & $(0.0777)$ & $(0)$ & (0) & - \\
\hline \multirow[t]{2}{*}{$(100,0.9,100)$} & 0.1500 & 0.2361 & 0.2256 & 0.1399 & 0.1858 & 0.1669 & 0 & 2 & 1 \\
\hline & $(0.1123)$ & $(0.1071)$ & $(0.1175)$ & $(0.1215)$ & $(0.0900)$ & $(0.1034)$ & (0) & (0) & - \\
\hline \multirow[t]{2}{*}{$(100,0.9,300)$} & 0.1503 & 0.2353 & 0.2319 & 0.1401 & 0.1876 & 0.1876 & 0 & 2 & 1 \\
\hline & $(0.1086)$ & $(0.1120)$ & $(0.1243)$ & $(0.1121)$ & $(0.0926)$ & $(0.1029)$ & $(0)$ & (0) & - \\
\hline \multirow[t]{2}{*}{$(100,0.9,600)$} & 0.1500 & 0.2236 & 0.2312 & 0.1360 & 0.2076 & 0.1858 & 0 & 2 & 1 \\
\hline & $(0.1175)$ & $(0.1195)$ & $(0.1121)$ & $(0.1076)$ & $(0.1124)$ & $(0.1101)$ & $(0)$ & $(0)$ & - \\
\hline \multirow[t]{2}{*}{$(200,0.7,100)$} & 0.0753 & 0.1276 & 0.1213 & 0.0733 & 0.1105 & 0.0887 & 0.015 & 2 & 0.985 \\
\hline & $(0.0569)$ & $(0.0480)$ & $(0.0487)$ & $(0.0572)$ & $(0.0393)$ & $(0.0504)$ & $(0.121)$ & (0) & - \\
\hline \multirow[t]{2}{*}{$(200,0.7,300)$} & 0.0836 & 0.1232 & 0.1256 & 0.0776 & 0.1075 & 0.0877 & 0.005 & 2 & 0.995 \\
\hline & $(0.0564)$ & $(0.0432)$ & $(0.0500)$ & $(0.0604)$ & $(0.0362)$ & $(0.0426)$ & $(0.070)$ & (0) & - \\
\hline \multirow[t]{2}{*}{$(200,0.7,600)$} & 0.0721 & 0.1288 & 0.1251 & 0.0789 & 0.1056 & 0.0920 & 0 & 2 & 1 \\
\hline & $(0.0571)$ & $(0.0409)$ & $(0.0523)$ & $(0.0581)$ & $(0.0350)$ & $(0.0497)$ & $(0)$ & (0) & - \\
\hline \multirow[t]{2}{*}{$(200,0.9,100)$} & 0.1068 & 0.1739 & 0.1657 & 0.0943 & 0.1463 & 0.1273 & 0.050 & 2 & 0.965 \\
\hline & $(0.0803)$ & $(0.0730)$ & $(0.0783)$ & $(0.0749)$ & $(0.0662)$ & $(0.0690)$ & $(0.279)$ & (0) & - \\
\hline \multirow[t]{2}{*}{$(200,0.9,300)$} & 0.1187 & 0.1712 & 0.1760 & 0.1042 & 0.1496 & 0.1286 & 0 & 2 & 1 \\
\hline & $(0.0826)$ & $(0.0751)$ & $(0.0807)$ & $(0.0774)$ & $(0.0554)$ & $(0.0690)$ & (0) & (0) & - \\
\hline \multirow[t]{2}{*}{$(200,0.9,600)$} & 0.1088 & 0.1778 & 0.1696 & 0.1034 & 0.1438 & 0.1293 & 0 & 2 & 1 \\
\hline & $(0.0835)$ & $(0.0785)$ & $(0.0846)$ & $(0.0788)$ & $(0.0633)$ & $(0.0772)$ & $(0)$ & $(0)$ & - \\
\hline \multirow[t]{2}{*}{$(300,0.7,100)$} & 0.0576 & 0.1094 & 0.1046 & 0.0586 & 0.0956 & 0.0693 & 0.025 & 2 & 0.975 \\
\hline & $(0.0453)$ & $(0.0344)$ & $(0.0341)$ & $(0.0437)$ & $(0.0267)$ & $(0.0366)$ & $(0.156)$ & (0) & - \\
\hline \multirow[t]{2}{*}{$(300,0.7,300)$} & 0.0596 & 0.1079 & 0.1127 & 0.0640 & 0.0963 & 0.0681 & 0.005 & 2 & 0.995 \\
\hline & $(0.0497)$ & $(0.0314)$ & $(0.0382)$ & $(0.0438)$ & $(0.0270)$ & $(0.0384)$ & $(0.070)$ & (0) & - \\
\hline \multirow[t]{2}{*}{$(300,0.7,600)$} & 0.0659 & 0.1122 & 0.1014 & 0.0651 & 0.0941 & 0.0689 & 0 & 2 & 1 \\
\hline & $(0.0490)$ & $(0.0366)$ & $(0.0369)$ & $(0.0463)$ & $(0.0280)$ & $(0.0367)$ & $(0)$ & (0) & - \\
\hline \multirow[t]{2}{*}{$(300,0.9,100)$} & 0.0876 & 0.1489 & 0.1448 & 0.0818 & 0.1271 & 0.1062 & 0.015 & 2 & 0.985 \\
\hline & $(0.0710)$ & $(0.0606)$ & $(0.0623)$ & $(0.0614)$ & $(0.0484)$ & $(0.0617)$ & $(0.121)$ & (0) & - \\
\hline \multirow[t]{2}{*}{$(300,0.9,300)$} & 0.0905 & 0.1432 & 0.1490 & 0.0831 & 0.1196 & 0.1057 & 0 & 2 & 1 \\
\hline & $(0.0697)$ & $(0.0566)$ & $(0.0605)$ & $(0.0594)$ & $(0.0404)$ & $(0.0548)$ & (0) & (0) & - \\
\hline \multirow[t]{2}{*}{$(300,0.9,600)$} & 0.0878 & 0.1517 & 0.1474 & 0.0800 & 0.1174 & 0.1039 & 0 & 2 & 1 \\
\hline & $(0.0666)$ & $(0.0663)$ & $(0.0648)$ & $(0.0592)$ & $(0.0422)$ & $(0.0501)$ & (0) & (0) & - \\
\hline
\end{tabular}



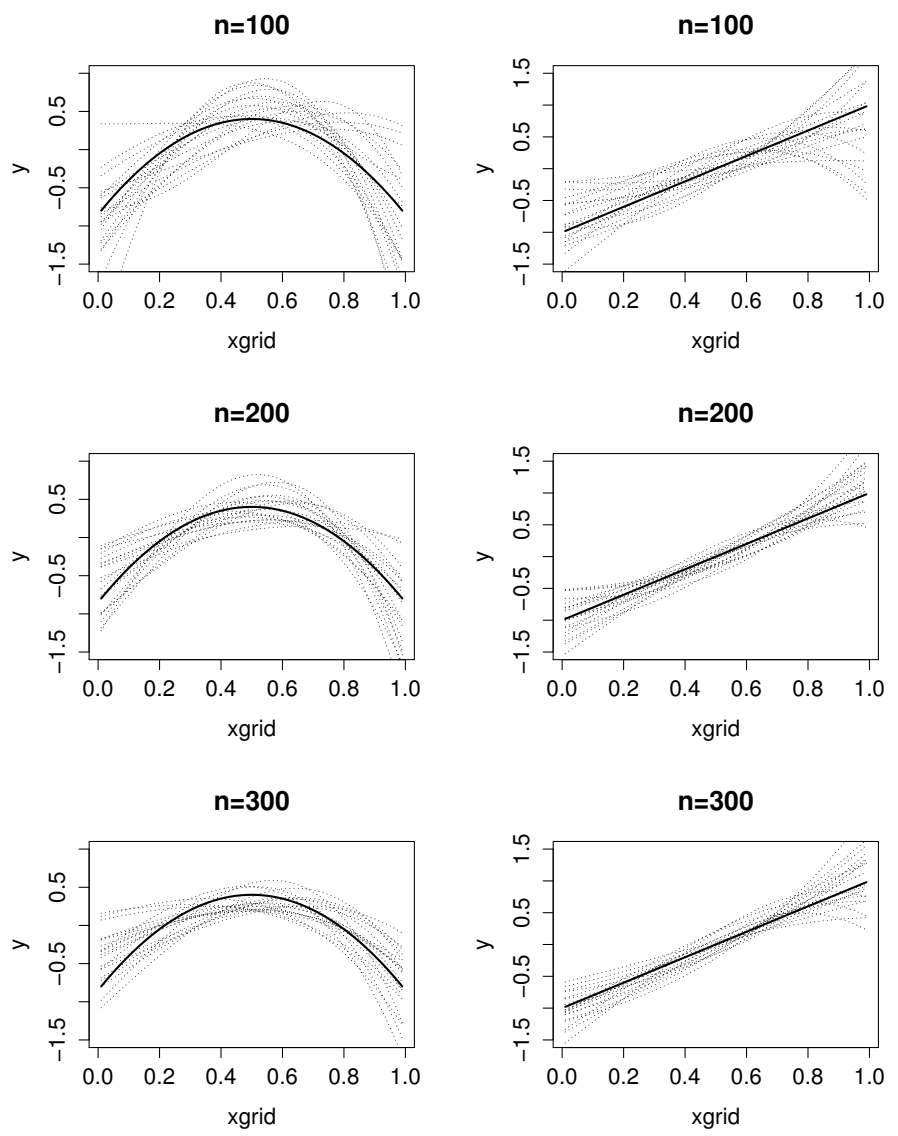

Figure 3.3: The SCAD penalized quantile estimates for $\kappa=1$ when the error follows a standard normal distribution. Left: Estimates for $\alpha_{4,1}(\cdot)$ when $\tau=0.5, p_{n}=300$; Right: Estimates for $\alpha_{15,2}(\cdot)$ when $\tau=0.7, p_{n}=100$. The solid curve is the true function and the dotted curves are the estimates for 20 randomly chosen replicates. 

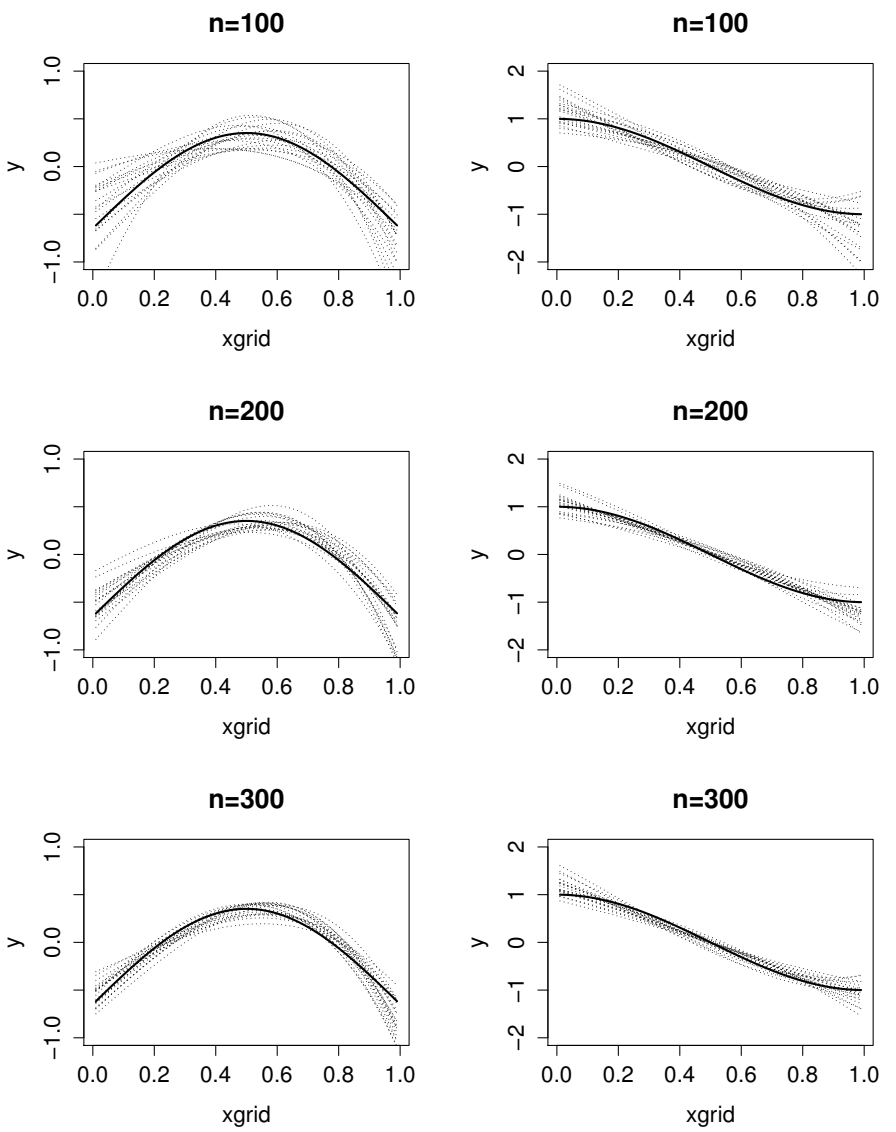

Figure 3.4: The SCAD penalized quantile estimates for $\kappa=1$ when the error follows a Student's t distribution with degrees of freedom 3 and scale parameter $1 / 3$. Left: Estimates for $\alpha_{4,2}(\cdot)$ when $\tau=0.5, p_{n}=600$; Right: Estimates for $\alpha_{15,1}(\cdot)$ when $\tau=0.9, p_{n}=100$. The solid curve is the true function and the dotted curves are the estimates for 20 randomly chosen replicates. 


\section{Chapter 3. Quantile Regression for Additive Coefficient Models 72 in High Dimensions}

(breed, gender, birth weight, weaning weight, 6-month weight, eye muscle area, fat thickness, pre-weaning gain, post-weaning gain, daily weight gain, height at withers, chest girth, and shin circumference) and 54,241 single nucleotide polymorphisms (SNPs). Zhang et al. (2013) applied linear models to identify the significant SNPs for 7 traits (weaning weight, 6-month weight, pre-weaning gain, post-weaning gain, daily weight gain, chest girth, and shin circumference) individually. No gene-environment $(\mathrm{G} \times \mathrm{E})$ interaction between SNPs and sheep traits was considered in their work. We are interested in selecting significant SNPs with environmental effects (breed, gender, birth weight) on the trait weaning weight. We eliminate the samples with more than 10\% SNPs missing. Furthermore, a SNP is excluded if all observations are missing, or its call rate is less than $90 \%$, or its minor allele frequency (MAF) is less than 5\%. The three genotypes are coded as $(1,1),(0,1)$ and $(1,0)$. To reduce the dimensionality, similar to Fan et al. (2014b), we perform a independence screening using one SNP at a time and keep the top 250 SNPs. After these processing steps, there are 327 samples and 250 SNPs left in our study.

We apply the dataset to the following additive coefficient model (ACM)

$$
Y_{i}=\sum_{l=0}^{500}\left\{\alpha_{l 0}+\sum_{s=1}^{4} \alpha_{l s}\left(X_{i s}\right)\right\} T_{i l}+\epsilon_{i}
$$

$i=1, \ldots, 327, Y_{i}$ is the weaning weight, $\left(X_{i 1}, X_{i 2}\right)$ is the sheep breed (taking three values and thus coded using two binary random variables), $X_{i 3}$ is the sheep gender (binary variable), $X_{i 4}$ is the birth weight. Since $X_{i 1}, X_{i 2}, X_{i 3}$ are binary, the corresponding coefficient functions in (3.10) are specified to be linear instead of being non-parametric, while the coefficient function on birth weight $\alpha_{l 3}\left(X_{i 3}\right)$ is non-parametric. We compare the additive coefficient model (ACM) (3.10) with a linear model (LM) at various quantile 
levels. Ten-fold cross-validation (CV) is used to select the tuning parameters $k_{n}$ and/or $\lambda_{n}$. The smallest CV error and the number of SNPs selected are shown in Table 3.9. The results show that additive coefficient model (3.10) performs better than the linear model for all 9 different quantile levels.

Table 3.9: Quantile regresstion analysis of weaning weight base on the sheep data.

\begin{tabular}{cccc}
\hline \hline$\tau$ & Model & Smallest CV error & Number of SNPs seleted \\
\hline 0.9 & ACM & 0.1283 & 6 \\
& LM & 0.1817 & 9 \\
\hline 0.7 & ACM & 0.2480 & 6 \\
& LM & 0.3353 & 4 \\
\hline 0.5 & ACM & 0.2803 & 14 \\
& LM & 0.3289 & 6 \\
\hline 0.3 & ACM & 0.2569 & 10 \\
& LM & 0.2752 & 9 \\
\hline 0.1 & ACM & 0.1411 & 5 \\
\hline \hline & LM & 0.1456 & 5 \\
\hline
\end{tabular}

\subsection{Summary}

In this chapter, we considered non-convex penalization for quantile additive coefficients models with high-dimensional covariates, and established the 


\section{Chapter 3. Quantile Regression for Additive Coefficient Models}

oracle property of the estimator. Our numerical results demonstrate the favourable performance of the proposed method.

\subsection{Appendix}

First we need the following additional notations. Let

$$
\begin{gathered}
\mathbf{D}_{i l}=\mathbf{\Pi}\left(\mathbf{X}_{i}\right) T_{i l}, \quad \mathbf{D}_{l}=\left(\mathbf{D}_{1 l}, \ldots, \mathbf{D}_{n l}\right)^{\mathrm{T}}, \quad l \in \mathcal{S}, \\
\mathbf{D}_{\mathcal{S}}=\left(\mathbf{D}_{l}, l \in \mathcal{S}\right), \quad \mathbf{H}_{n, \mathcal{S}}:=\mathbf{D}_{\mathcal{S}}^{\mathrm{T}} \mathbf{D}_{\mathcal{S}}, \\
\boldsymbol{\beta}_{\mathcal{S}}^{*}=\mathbf{H}_{n, \mathcal{S}}^{1 / 2} \boldsymbol{\beta}_{\mathcal{S}}, \quad \boldsymbol{\beta}_{0, \mathcal{S}}^{*}=\mathbf{H}_{n, \mathcal{S}}^{1 / 2} \boldsymbol{\beta}_{0, \mathcal{S}}, \quad \mathbf{Z}_{i, \mathcal{S}}:=\mathbf{H}_{n, \mathcal{S}}^{-1 / 2} \boldsymbol{\Pi}_{\mathcal{S}}\left(\mathbf{X}_{i}, \mathbf{T}_{i}\right) .
\end{gathered}
$$

Then the objective function in 3.3 can be written as

$$
\hat{\boldsymbol{\beta}}_{\mathcal{S}}^{*}=\underset{\boldsymbol{\beta}_{\mathcal{S}}^{*}}{\arg \min } \sum_{i=1}^{n} \rho_{\tau}\left(\epsilon_{i}-R_{i, \mathcal{S}}-\mathbf{Z}_{i, \mathcal{S}}^{\mathrm{T}}\left(\boldsymbol{\beta}_{\mathcal{S}}^{*}-\boldsymbol{\beta}_{0, \mathcal{S}}^{*}\right)\right) .
$$

In our proofs, $c$ and $C$ are generic positive constants, which may assume different values in different places.

Lemma 3.1. Under conditions (C1)-(C6), for any positive constant $L$, we have

$$
\begin{array}{r}
\sup _{\left\|\boldsymbol{\beta}_{\mathcal{S}}^{*}\right\| \leq L q_{n}^{1 / 2} k_{n}^{1 / 2}} q_{n}^{-1} k_{n}^{-1} \mid \sum_{i=1}^{n}\left[\rho_{\tau}\left(\epsilon_{i}-\mathbf{Z}_{i, \mathcal{S}}^{\mathrm{T}} \boldsymbol{\beta}_{\mathcal{S}}^{*}-R_{i, \mathcal{S}}\right)-\rho_{\tau}\left(\epsilon_{i}-R_{i, \mathcal{S}}\right)\right. \\
+\mathbf{Z}_{i, \mathcal{S}}^{\mathrm{T}} \boldsymbol{\beta}_{\mathcal{S}}^{*}\left(\tau-I\left(\epsilon_{i} \leq 0\right)\right)-\mathrm{E}\left(\rho_{\tau}\left(\epsilon_{i}-\mathbf{Z}_{i, \mathcal{S}}^{\mathrm{T}} \boldsymbol{\beta}_{\mathcal{S}}^{*}-R_{i, \mathcal{S}}\right)\right. \\
\left.\left.-\rho_{\tau}\left(\epsilon_{i}-R_{i, \mathcal{S}}\right)\right)\right] \mid=o_{p}(1) .
\end{array}
$$

Proof. To prove this lemma, it is sufficient to show for any $\epsilon>0$,

$$
\mathrm{P}\left(\sup _{\left\|\boldsymbol{\beta}_{\mathcal{S}}^{*}\right\| \leq 1} q_{n}^{-1} k_{n}^{-1}\left|\sum_{i=1}^{n} Q_{i}\left(\boldsymbol{\beta}_{\mathcal{S}}^{*}\right)\right|>\epsilon\right) \rightarrow 0
$$


where

$$
\begin{aligned}
Q_{i}\left(\boldsymbol{\beta}_{\mathcal{S}}^{*}\right)= & \rho_{\tau}\left(\epsilon_{i}-L q_{n}^{1 / 2} k_{n}^{1 / 2} \mathbf{Z}_{i, \mathcal{S}}^{\mathrm{T}} \boldsymbol{\beta}_{\mathcal{S}}^{*}-R_{i, \mathcal{S}}\right)-\rho_{\tau}\left(\epsilon_{i}-R_{i, \mathcal{S}}\right) \\
& +L q_{n}^{1 / 2} k_{n}^{1 / 2} \mathbf{Z}_{i, \mathcal{S}}^{\mathrm{T}} \boldsymbol{\beta}_{\mathcal{S}}^{*}\left(\tau-I\left(\epsilon_{i} \leq 0\right)\right) \\
& -\mathrm{E}\left(\rho_{\tau}\left(\epsilon_{i}-L q_{n}^{1 / 2} k_{n}^{1 / 2} \mathbf{Z}_{i, \mathcal{S}}^{\mathrm{T}} \boldsymbol{\beta}_{\mathcal{S}}^{*}-R_{i, \mathcal{S}}\right)-\rho_{\tau}\left(\epsilon_{i}-R_{i, \mathcal{S}}\right)\right) .
\end{aligned}
$$

As in He and Shi (1994), we let $\boldsymbol{\Theta}:=\left\{\boldsymbol{\beta}_{\mathcal{S}}^{*}:\left\|\boldsymbol{\beta}_{\mathcal{S}}^{*}\right\| \leq 1\right\}$ be the parameter space and $\boldsymbol{\Theta}_{1}, \ldots, \Theta_{K_{n}}$ be a covering of $\Theta$ with the diameter $R_{n}=\frac{\epsilon}{4 L C \sqrt{n}}$. By Lemma 2.5 in van der Geer (2000), this covering can be constructed with size $K_{n} \leq C\left(\frac{C \sqrt{n}}{\epsilon}\right)^{q_{n}\left(1+d J_{n}\right)}$. Let $\boldsymbol{\beta}_{\mathcal{S}, k}^{*} \in \boldsymbol{\Theta}_{k}, k=1, \ldots, K_{n}$. We have

$$
\begin{aligned}
& \mathrm{P}\left(\sup _{\left\|\boldsymbol{\beta}_{\mathcal{S}}^{*}\right\| \leq 1} q_{n}^{-1} k_{n}^{-1}\left|\sum_{i=1}^{n} Q_{i}\left(\boldsymbol{\beta}_{\mathcal{S}}^{*}\right)\right|>\epsilon\right) \\
\leq & \sum_{k=1}^{K_{n}} \mathrm{P}\left(\sup _{\boldsymbol{\beta}_{\mathcal{S}}^{*} \in \boldsymbol{\Theta}_{k}} q_{n}^{-1} k_{n}^{-1}\left|\sum_{i=1}^{n} Q_{i}\left(\boldsymbol{\beta}_{\mathcal{S}}^{*}\right)\right|>\epsilon\right) \\
\leq & \sum_{k=1}^{K_{n}} \mathrm{P}\left(\left(q_{n}^{-1} k_{n}^{-1}\left|\sum_{i=1}^{n} Q_{i}\left(\boldsymbol{\beta}_{\mathcal{S}, k}^{*}\right)\right|\right.\right. \\
& \left.\left.+\sup _{\boldsymbol{\beta}_{\mathcal{S}}^{*} \in \boldsymbol{\Theta}_{k}} q_{n}^{-1} k_{n}^{-1}\left|\sum_{i=1}^{n}\left(Q_{i}\left(\boldsymbol{\beta}_{\mathcal{S}}^{*}\right)-Q_{i}\left(\boldsymbol{\beta}_{\mathcal{S}, k}^{*}\right)\right)\right|\right)>\epsilon\right) .
\end{aligned}
$$

We first show, for any $k=1, \ldots, K_{n}$,

$$
\sup _{\boldsymbol{\beta}_{\mathcal{S}}^{*} \in \boldsymbol{\Theta}_{k}} q_{n}^{-1} k_{n}^{-1}\left|\sum_{i=1}^{n}\left(Q_{i}\left(\boldsymbol{\beta}_{\mathcal{S}}^{*}\right)-Q_{i}\left(\boldsymbol{\beta}_{\mathcal{S}, k}^{*}\right)\right)\right| \leq \epsilon / 2 .
$$

Noting that $\rho_{\tau}(u)=|u| / 2+(\tau-1 / 2) u$, we have

$$
\begin{aligned}
& \left|Q_{i}\left(\boldsymbol{\beta}_{\mathcal{S}}^{*}\right)-Q_{i}\left(\boldsymbol{\beta}_{\mathcal{S}, k}^{*}\right)\right| \\
= & || \epsilon_{i}-L q_{n}^{1 / 2} k_{n}^{1 / 2} \mathbf{Z}_{i, \mathcal{S}}^{\mathrm{T}} \boldsymbol{\beta}_{\mathcal{S}}^{*}-R_{i, \mathcal{S}}|/ 2-| \epsilon_{i}-L q_{n}^{1 / 2} k_{n}^{1 / 2} \mathbf{Z}_{i, \mathcal{S}}^{\mathrm{T}} \boldsymbol{\beta}_{\mathcal{S}, k}^{*}-R_{i, \mathcal{S}} \mid / 2 \\
& +L q_{n}^{1 / 2} k_{n}^{1 / 2} \mathbf{Z}_{i, \mathcal{S}}^{\mathrm{T}}\left(\boldsymbol{\beta}_{\mathcal{S}}^{*}-\boldsymbol{\beta}_{\mathcal{S}, k}^{*}\right)\left(\tau-I\left(\epsilon_{i} \leq 0\right)\right)-\mathrm{E}\left(\mid \epsilon_{i}-L q_{n}^{1 / 2} k_{n}^{1 / 2} \mathbf{Z}_{i, \mathcal{S}}^{\mathrm{T}} \boldsymbol{\beta}_{\mathcal{S}}^{*}\right. \\
& \left.-R_{i, \mathcal{S}}|/ 2-| \epsilon_{i}-L q_{n}^{1 / 2} k_{n}^{1 / 2} \mathbf{Z}_{i, \mathcal{S}}^{\mathrm{T}} \boldsymbol{\beta}_{\mathcal{S}, k}^{*}-R_{i, \mathcal{S}} \mid / 2\right) \mid \\
\leq & 2 L q_{n}^{1 / 2} k_{n}^{1 / 2}\left|\mathbf{Z}_{i, \mathcal{S}}^{\mathrm{T}}\left(\boldsymbol{\beta}_{\mathcal{S}}^{*}-\boldsymbol{\beta}_{\mathcal{S}, k}^{*}\right)\right| .
\end{aligned}
$$


Therefore,

$$
\begin{aligned}
& \sup _{\boldsymbol{\beta}_{\mathcal{S}}^{*} \in \boldsymbol{\Theta}_{k}} q_{n}^{-1} k_{n}^{-1}\left|\sum_{i=1}^{n}\left(Q_{i}\left(\boldsymbol{\beta}_{\mathcal{S}}^{*}\right)-Q_{i}\left(\boldsymbol{\beta}_{\mathcal{S}, k}^{*}\right)\right)\right| \\
\leq & 2 L \sup _{\boldsymbol{\beta}_{\mathcal{S}}^{*} \in \boldsymbol{\Theta}_{k}} q_{n}^{-1 / 2} k_{n}^{-1 / 2} \sum_{i=1}^{n}\left|\mathbf{Z}_{i, \mathcal{S}}^{\mathrm{T}}\left(\boldsymbol{\beta}_{\mathcal{S}}^{*}-\boldsymbol{\beta}_{\mathcal{S}, k}^{*}\right)\right| \\
\leq & 2 L C q_{n}^{-1 / 2} k_{n}^{-1 / 2} n \sqrt{q_{n} k_{n} / n} R_{n} \\
= & 2 L C n^{1 / 2} R_{n}=\epsilon / 2 .
\end{aligned}
$$

Next, we prove $\sum_{k=1}^{K_{n}} \mathrm{P}\left(q_{n}^{-1} k_{n}^{-1}\left|\sum_{i=1}^{n} Q_{i}\left(\boldsymbol{\beta}_{\mathcal{S}, k}^{*}\right)\right|>\epsilon / 2\right) \rightarrow 0$. We have

$$
\begin{aligned}
& \max _{1 \leq i \leq n}\left|Q_{i}\left(\boldsymbol{\beta}_{\mathcal{S}, k}^{*}\right)\right| \\
= & \max _{1 \leq i \leq n}|| \epsilon_{i}-L q_{n}^{1 / 2} k_{n}^{1 / 2} \mathbf{Z}_{i, \mathcal{S}}^{\mathrm{T}} \boldsymbol{\beta}_{\mathcal{S}, k}^{*}-R_{i, \mathcal{S}}|/ 2-| \epsilon_{i}-R_{i, \mathcal{S}} \mid / 2 \\
& +L q_{n}^{1 / 2} k_{n}^{1 / 2} \mathbf{Z}_{i, \mathcal{S}}^{\mathrm{T}} \boldsymbol{\beta}_{\mathcal{S}, k}^{*}\left(\tau-I\left(\epsilon_{i} \leq 0\right)\right) \\
& -\mathrm{E}\left(\left|\epsilon_{i}-L q_{n}^{1 / 2} k_{n}^{1 / 2} \mathbf{Z}_{i, \mathcal{S}}^{\mathrm{T}} \boldsymbol{\beta}_{\mathcal{S}, k}^{*}-R_{i, \mathcal{S}}\right| / 2-\left|\epsilon_{i}-R_{i, \mathcal{S}}\right| / 2\right) \mid \\
\leq & 2 L q_{n}^{1 / 2} k_{n}^{1 / 2} \max _{1 \leq i \leq n}\left|\mathbf{Z}_{i, \mathcal{S}}^{\mathrm{T}} \boldsymbol{\beta}_{\mathcal{S}, k}^{*}\right| \\
\leq & 2 C L q_{n} k_{n} n^{-1 / 2} .
\end{aligned}
$$

Let

$$
\begin{aligned}
\tilde{Q}_{i}\left(\boldsymbol{\beta}_{\mathcal{S}, k}^{*}\right):= & \rho_{\tau}\left(\epsilon_{i}-L q_{n}^{1 / 2} k_{n}^{1 / 2} \mathbf{Z}_{i, \mathcal{S}}^{\mathrm{T}} \boldsymbol{\beta}_{\mathcal{S}, k}^{*}-R_{i, \mathcal{S}}\right)-\rho_{\tau}\left(\epsilon_{i}-R_{i, \mathcal{S}}\right) \\
& +L q_{n}^{1 / 2} k_{n}^{1 / 2} \mathbf{Z}_{i, \mathcal{S}}^{\mathrm{T}} \boldsymbol{\beta}_{\mathcal{S}, k}^{*}\left(\tau-I\left(\epsilon_{i} \leq 0\right)\right) .
\end{aligned}
$$

Then, $Q_{i}\left(\boldsymbol{\beta}_{\mathcal{S}, k}^{*}\right)=\tilde{Q}_{i}\left(\boldsymbol{\beta}_{\mathcal{S}, k}^{*}\right)-\mathrm{E} \tilde{Q}_{i}\left(\boldsymbol{\beta}_{\mathcal{S}, k}^{*}\right), \mathrm{E} Q_{i}\left(\boldsymbol{\beta}_{\mathcal{S}, k}^{*}\right)=0$ and $\operatorname{Var}\left(Q_{i}\left(\boldsymbol{\beta}_{\mathcal{S}, k}^{*}\right)\right)=$ $\operatorname{Var}\left(\tilde{Q}_{i}\left(\boldsymbol{\beta}_{\mathcal{S}, k}^{*}\right)\right)$. Using Knight's identity

$$
\rho_{\tau}(r-s)-\rho_{\tau}(r)=-s(\tau-I(r \leq 0))+\int_{0}^{s}(I(r \leq t)-I(r \leq 0)) d t,
$$

we have

$$
\tilde{Q}_{i}\left(\boldsymbol{\beta}_{\mathcal{S}, k}^{*}\right)
$$




$$
\begin{aligned}
= & L q_{n}^{1 / 2} k_{n}^{1 / 2} \mathbf{Z}_{i, \mathcal{S}}^{\mathrm{T}} \boldsymbol{\beta}_{\mathcal{S}, k}^{*}\left(I\left(\epsilon_{i}-R_{i, \mathcal{S}} \leq 0\right)-I\left(\epsilon_{i} \leq 0\right)\right) \\
& +\int_{0}^{L q_{n}^{1 / 2} k_{n}^{1 / 2} \mathbf{Z}_{i, \mathcal{S}}^{\mathrm{T}} \boldsymbol{\beta}_{\mathcal{S}, k}^{*}}\left(I\left(\epsilon_{i}-R_{i, \mathcal{S}} \leq t\right)-I\left(\epsilon_{i}-R_{i, \mathcal{S}} \leq 0\right)\right) d t \\
:= & \tilde{Q}_{i, 1}\left(\boldsymbol{\beta}_{\mathcal{S}, k}^{*}\right)+\tilde{Q}_{i, 2}\left(\boldsymbol{\beta}_{\mathcal{S}, k}^{*}\right) .
\end{aligned}
$$

Thus,

$$
\begin{aligned}
\operatorname{Var}\left(\tilde{Q}_{i}\left(\boldsymbol{\beta}_{\mathcal{S}, k}^{*}\right)\right) & =\operatorname{Var}\left(\tilde{Q}_{i, 1}\left(\boldsymbol{\beta}_{\mathcal{S}, k}^{*}\right)+\tilde{Q}_{i, 2}\left(\boldsymbol{\beta}_{\mathcal{S}, k}^{*}\right)\right) \\
& \leq \mathrm{E} \tilde{Q}_{i, 1}\left(\boldsymbol{\beta}_{\mathcal{S}, k}^{*}\right)^{2}+\mathrm{E} \tilde{Q}_{i, 2}\left(\boldsymbol{\beta}_{\mathcal{S}, k}^{*}\right)^{2}
\end{aligned}
$$

We have

$$
\begin{aligned}
& \sum_{i=1}^{n} \mathrm{E} \tilde{Q}_{i, 1}\left(\boldsymbol{\beta}_{\mathcal{S}, k}^{*}\right)^{2} \\
= & L^{2} q_{n} k_{n} \sum_{i=1}^{n} \mathrm{E}\left(\mathbf{Z}_{i, \mathcal{S}}^{\mathrm{T}} \boldsymbol{\beta}_{\mathcal{S}, k}^{*}\right)^{2}\left|I\left(\epsilon_{i}-R_{i, \mathcal{S}} \leq 0\right)-I\left(\epsilon_{i} \leq 0\right)\right| \\
\leq & L^{2} C^{2} q_{n}^{2} k_{n}^{2} n^{-1} \sum_{i=1}^{n} \mathrm{E} I\left(0 \leq\left|\epsilon_{i}\right| \leq\left|R_{i, \mathcal{S}}\right|\right) \\
= & L^{2} C^{2} q_{n}^{2} k_{n}^{2} n^{-1} \sum_{i=1}^{n} \int_{-\left|R_{i, \mathcal{S}}\right|}^{\left|R_{i, \mathcal{S}}\right|} f_{i}(s) d s \\
\leq & C q_{n}^{3} k_{n}^{2-r} .
\end{aligned}
$$

Furthermore, since $\left|\tilde{Q}_{i, 2}\left(\boldsymbol{\beta}_{\mathcal{S}, k}^{*}\right)\right| \leq C q_{n} k_{n} n^{-1 / 2}$, we have

$$
\begin{aligned}
& \sum_{i=1}^{n} \mathrm{E} \tilde{Q}_{i, 2}\left(\boldsymbol{\beta}_{\mathcal{S}, k}^{*}\right)^{2} \\
\leq & C q_{n} k_{n} n^{-1 / 2} \sum_{i=1}^{n} \mathrm{E} \int_{0}^{L q_{n}^{1 / 2} k_{n}^{1 / 2} \mathbf{z}_{i, \mathcal{S}}^{\mathrm{T}} \boldsymbol{\beta}_{\mathcal{S}, k}^{*}}\left(I\left(\epsilon_{i}-R_{i, \mathcal{S}} \leq t\right)\right. \\
& \left.-I\left(\epsilon_{i}-R_{i, \mathcal{S}} \leq 0\right)\right) d t \\
= & C q_{n} k_{n} n^{-1 / 2} \sum_{i=1}^{n} \int_{0}^{L q_{n}^{1 / 2} k_{n}^{1 / 2} \mathbf{z}_{i, \mathcal{S}}^{\mathrm{T}} \boldsymbol{\beta}_{\mathcal{S}, k}^{*}}\left(F_{i}\left(t+R_{i, \mathcal{S}}\right)-F_{i}\left(R_{i, \mathcal{S}}\right)\right) d t \\
= & C q_{n} k_{n} n^{-1 / 2} \sum_{i=1}^{n} \int_{0}^{L q_{n}^{1 / 2} k_{n}^{1 / 2} \mathbf{z}_{i, \mathcal{S}}^{\mathrm{T}} \boldsymbol{\beta}_{\mathcal{S}, k}^{*}}\left(f_{i}(0)+o(1)\right)\left(t+O\left(t^{2}\right)\right) d t
\end{aligned}
$$




$$
\begin{aligned}
& \leq C q_{n} k_{n} n^{-1 / 2} \sum_{i=1}^{n} L^{2} q_{n} k_{n} \boldsymbol{\beta}_{\mathcal{S}, k}^{* T} \mathbf{Z}_{i, \mathcal{S}} \mathbf{Z}_{i, \mathcal{S}}^{\mathrm{T}} \boldsymbol{\beta}_{\mathcal{S}, k}^{*} \\
& \leq C q_{n}^{2} k_{n}^{2} n^{-1 / 2}
\end{aligned}
$$

Therefore, $\sum_{i=1}^{n} \operatorname{Var}\left(Q_{i}\left(\boldsymbol{\beta}_{\mathcal{S}, k}^{*}\right)\right) \leq C q_{n}^{3} k_{n}^{2-r}$. By Bernstein's inequality, for sufficiently large $n$,

$$
\begin{aligned}
& \sum_{k=1}^{K_{n}} \mathrm{P}\left(q_{n}^{-1} k_{n}^{-1}\left|\sum_{i=1}^{n} Q_{i}\left(\boldsymbol{\beta}_{\mathcal{S}, k}^{*}\right)\right|>\epsilon / 2\right) \\
= & \sum_{k=1}^{K_{n}} \mathrm{P}\left(\left|\sum_{i=1}^{n} Q_{i}\left(\boldsymbol{\beta}_{\mathcal{S}, k}^{*}\right)\right|>q_{n} k_{n} \epsilon / 2\right) \\
\leq & \sum_{k=1}^{K_{n}} \exp \left(\frac{-q_{n}^{2} k_{n}^{2} \epsilon^{2} / 4}{C q_{n}^{3} k_{n}^{2-r}+2 C L \epsilon q_{n}^{2} k_{n}^{2} n^{-1 / 2}}\right) \\
\leq & 2 K_{n} \exp \left(-C q_{n}^{-1} k_{n}^{r}\right) \\
\leq & C \exp \left(C q_{n} k_{n} \log (n)-C q_{n}^{-1} k_{n}^{r}\right) .
\end{aligned}
$$

From conditions (C1), (C2) and (C5), we have

$$
\sum_{k=1}^{K_{n}} \mathrm{P}\left(q_{n}^{-1} k_{n}^{-1}\left|\sum_{i=1}^{n} Q_{i}\left(\boldsymbol{\beta}_{\mathcal{S}, k}^{*}\right)\right|>\epsilon / 2\right) \rightarrow 0,
$$

and we complete the proof.

Lemma 3.2. Under conditions (C1)-(C6), for any $\epsilon>0$, there exists $L$ such that as $n \rightarrow \infty$,

$$
\begin{aligned}
\mathrm{P}\left\{q _ { n } ^ { - 1 } k _ { n } ^ { - 1 } \left(\inf _{\left\|\boldsymbol{\beta}_{\mathcal{S}}^{*}\right\|=L q_{n}^{1 / 2} k_{n}^{1 / 2}} \sum_{i=1}^{n}[\right.\right. & {\left[\mathrm{E}\left(\rho_{\tau}\left(\epsilon_{i}-\mathbf{Z}_{i, \mathcal{S}}^{\mathrm{T}} \boldsymbol{\beta}_{\mathcal{S}}^{*}-R_{i, \mathcal{S}}\right)-\rho_{\tau}\left(\epsilon_{i}-R_{i, \mathcal{S}}\right)\right)\right] } \\
& \left.\left.-\sum_{i=1}^{n} \mathbf{Z}_{i, \mathcal{S}}^{\mathrm{T}} \boldsymbol{\beta}_{\mathcal{S}}^{*}\left(\tau-I\left(\epsilon_{i} \leq 0\right)\right)\right)>1\right\}>1-\epsilon .
\end{aligned}
$$

Proof. From Knight's identity, we have

$$
q_{n}^{-1} k_{n}^{-1} \sum_{i=1}^{n} \mathrm{E}\left(\rho_{\tau}\left(\epsilon_{i}-\mathbf{Z}_{i, \mathcal{S}}^{\mathrm{T}} \boldsymbol{\beta}_{\mathcal{S}}^{*}-R_{i, \mathcal{S}}\right)-\rho_{\tau}\left(\epsilon_{i}-R_{i, \mathcal{S}}\right)\right)
$$




$$
\begin{aligned}
& =q_{n}^{-1} k_{n}^{-1} \sum_{i=1}^{n} \mathrm{E} \int_{0}^{\mathbf{Z}_{i, \mathcal{S}}^{\mathrm{T}} \boldsymbol{\beta}_{\mathcal{S}}^{*}}\left(I\left(\epsilon_{i}-R_{i, \mathcal{S}} \leq t\right)-I\left(\epsilon_{i}-R_{i, \mathcal{S}} \leq 0\right)\right) d t \\
& =q_{n}^{-1} k_{n}^{-1} \sum_{i=1}^{n} \int_{0}^{\mathbf{Z}_{i, \mathcal{S}}^{\mathrm{T}} \boldsymbol{\beta}_{\mathcal{S}}^{*}}\left(F_{i}\left(t+R_{i, \mathcal{S}}\right)-F_{i}\left(R_{i, \mathcal{S}}\right)\right) d t \\
& =q_{n}^{-1} k_{n}^{-1} \sum_{i=1}^{n} \int_{0}^{\mathbf{Z}_{i, \mathcal{S}}^{\mathrm{T}} \boldsymbol{\beta}_{\mathcal{S}}^{*}}\left(f_{i}(0)+o(1)\right)\left(t+O\left(t^{2}\right)\right) d t \\
& =q_{n}^{-1} k_{n}^{-1}\left(f_{i}(0)+o(1)\right) / 2 \sum_{i=1}^{n} \boldsymbol{\beta}_{\mathcal{S}}^{* T} \mathbf{Z}_{i, \mathcal{S}} \mathbf{Z}_{i, \mathcal{S}}^{\mathrm{T}} \boldsymbol{\beta}_{\mathcal{S}}^{*},
\end{aligned}
$$

with probability approaching 1 . Therefore, there exits a constant $C>0$ such that

$$
q_{n}^{-1} k_{n}^{-1}\left(f_{i}(0)+o(1)\right) / 2 \sum_{i=1}^{n} \boldsymbol{\beta}_{\mathcal{S}}^{* T} \mathbf{Z}_{i, \mathcal{S}} \mathbf{Z}_{i, \mathcal{S}}^{\mathrm{T}} \boldsymbol{\beta}_{\mathcal{S}}^{*}=C q_{n}^{-1} k_{n}^{-1}\left\|\boldsymbol{\beta}_{\mathcal{S}}^{*}\right\|^{2}=C L .
$$

Next, we consider $\sum_{i=1}^{n} \mathbf{Z}_{i, \mathcal{S}}^{\mathrm{T}} \boldsymbol{\beta}_{\mathcal{S}}^{*}\left(\tau-I\left(\epsilon_{i} \leq 0\right)\right)$. Note that

$$
\mathrm{E} \sum_{i=1}^{n} \mathbf{Z}_{i, \mathcal{S}}^{\mathrm{T}} \boldsymbol{\beta}_{\mathcal{S}}^{*}\left(\tau-I\left(\epsilon_{i} \leq 0\right)\right)=0
$$

and

$$
\mathrm{E}\left(\sum_{i=1}^{n} \mathbf{Z}_{i, \mathcal{S}}^{\mathrm{T}} \boldsymbol{\beta}_{\mathcal{S}}^{*}\left(\tau-I\left(\epsilon_{i} \leq 0\right)\right)\right)^{2} \leq C\left\|\boldsymbol{\beta}_{\mathcal{S}}^{*}\right\|^{2},
$$

since $\mathrm{E}\left(\tau-I\left(\epsilon_{i} \leq 0\right)\right)\left(\tau-I\left(\epsilon_{j} \leq 0\right)\right)=0$ for $i \neq j$. Therefore, we have

$$
q_{n}^{-1} k_{n}^{-1} \sum_{i=1}^{n} \mathbf{Z}_{i, \mathcal{S}}^{\mathrm{T}} \boldsymbol{\beta}_{\mathcal{S}}^{*}\left(\tau-I\left(\epsilon_{i} \leq 0\right)\right) \leq C L q_{n}^{-1 / 2} k_{n}^{-1 / 2} \rightarrow 0,
$$

which completes the proof.

Lemma 3.3. Under Conditions (C1)-(C7), if $q_{n} k_{n}^{3 / 2} n^{-1 / 2}=o\left(\lambda_{n}\right)$ and $n \lambda_{n} /\left(k_{n}^{1 / 2} \log \left(p_{n}\right)\right) \rightarrow \infty$, we have

$$
\begin{array}{r}
\mathrm{P}\left(\max _{l \in \mathcal{S}^{c}} \sup _{\left\|\boldsymbol{\beta}_{\mathcal{S}}-\boldsymbol{\beta}_{0, \mathcal{S}}\right\| \leq C q_{n}^{1 / 2} k_{n} n^{-1 / 2}} \| \sum_{i=1}^{n}\left(I\left(Y_{i}-\boldsymbol{\Pi}_{\mathcal{S}}\left(\mathbf{X}_{i}, \mathbf{T}_{i}\right)^{\mathrm{T}} \boldsymbol{\beta}_{\mathcal{S}} \leq 0\right)\right.\right. \\
-I\left(Y_{i}-\boldsymbol{\Pi}_{\mathcal{S}}\left(\mathbf{X}_{i}, \mathbf{T}_{i}\right)^{\mathrm{T}} \boldsymbol{\beta}_{0, \mathcal{S}} \leq 0\right)-\mathrm{P}\left(Y_{i}-\boldsymbol{\Pi}_{\mathcal{S}}\left(\mathbf{X}_{i}, \mathbf{T}_{i}\right)^{\mathrm{T}} \boldsymbol{\beta}_{\mathcal{S}} \leq 0\right) \\
\left.\left.+\mathrm{P}\left(Y_{i}-\boldsymbol{\Pi}_{\mathcal{S}}\left(\mathbf{X}_{i}, \mathbf{T}_{i}\right)^{\mathrm{T}} \boldsymbol{\beta}_{0, \mathcal{S}} \leq 0\right)\right) T_{i l} \boldsymbol{\Pi}\left(\mathbf{X}_{i}\right) \|>c n \lambda_{n}\right) \rightarrow 0
\end{array}
$$




\section{Chapter 3. Quantile Regression for Additive Coefficient Models}

for some positive constants $C$ and $c$.

Proof. Let $\boldsymbol{\Theta}:=\left\{\boldsymbol{\beta}_{\mathcal{S}}:\left\|\boldsymbol{\beta}_{\mathcal{S}}-\boldsymbol{\beta}_{0, \mathcal{S}}\right\| \leq C q_{n}^{1 / 2} k_{n} n^{-1 / 2}\right\}$ be the parameter space. As in Sherwood and Wang (2016), we construct a covering $\boldsymbol{\Theta}_{1}, \ldots, \boldsymbol{\Theta}_{K_{n}}$ of $\boldsymbol{\Theta}$, with centres $\boldsymbol{\beta}_{\mathcal{S}, j} \in \boldsymbol{\Theta}_{j}, j=1, \ldots, K_{n}$, the diameters bounded by $R_{n}=q_{n}^{1 / 2} k_{n} n^{-5 / 2}$, and size $K_{n} \leq n^{2 q_{n}\left(1+d J_{n}\right)}$. Note that $\epsilon_{i}=Y_{i}-\boldsymbol{\Pi}_{\mathcal{S}}\left(X_{i}, T_{i}\right)^{\mathrm{T}} \boldsymbol{\beta}_{0, \mathcal{S}}+R_{i, \mathcal{S}}$. Let $\epsilon_{i}\left(\boldsymbol{\beta}_{\mathcal{S}}\right)=Y_{i}-\boldsymbol{\Pi}_{\mathcal{S}}\left(X_{i}, T_{i}\right)^{\mathrm{T}} \boldsymbol{\beta}_{\mathcal{S}}+R_{i, \mathcal{S}}$.

We have

$$
\begin{aligned}
& \mathrm{P}\left(\sup _{\left\|\boldsymbol{\beta}_{\mathcal{S}}-\boldsymbol{\beta}_{0, \mathcal{S}}\right\| \leq C q_{n}^{1 / 2} k_{n} n^{-1 / 2}} \| \sum_{i=1}^{n}\left(I\left(Y_{i}-\boldsymbol{\Pi}_{\mathcal{S}}\left(\mathbf{X}_{i}, \mathbf{T}_{i}\right)^{\mathrm{T}} \boldsymbol{\beta}_{\mathcal{S}} \leq 0\right)\right.\right. \\
& -I\left(Y_{i}-\boldsymbol{\Pi}_{\mathcal{S}}\left(\mathbf{X}_{i}, \mathbf{T}_{i}\right)^{\mathrm{T}} \boldsymbol{\beta}_{0, \mathcal{S}} \leq 0\right)-\mathrm{P}\left(Y_{i}-\boldsymbol{\Pi}_{\mathcal{S}}\left(\mathbf{X}_{i}, \mathbf{T}_{i}\right)^{\mathrm{T}} \boldsymbol{\beta}_{\mathcal{S}} \leq 0\right) \\
& \left.\left.+\mathrm{P}\left(Y_{i}-\boldsymbol{\Pi}_{\mathcal{S}}\left(\mathbf{X}_{i}, \mathbf{T}_{i}\right)^{\mathrm{T}} \boldsymbol{\beta}_{0, \mathcal{S}} \leq 0\right)\right) T_{i l} \boldsymbol{\Pi}\left(\mathbf{X}_{i}\right) \|>c n \lambda_{n}\right) \\
& =\mathrm{P}\left(\sup _{\left\|\boldsymbol{\beta}_{\mathcal{S}}-\boldsymbol{\beta}_{0, \mathcal{S}}\right\| \leq C q_{n}^{1 / 2} k_{n} n^{-1 / 2}} \| \sum_{i=1}^{n}\left(I\left(\epsilon_{i}\left(\boldsymbol{\beta}_{\mathcal{S}}\right) \leq R_{i, \mathcal{S}}\right)-I\left(\epsilon_{i} \leq R_{i, \mathcal{S}}\right)\right.\right. \\
& \left.\left.-\mathrm{P}\left(\epsilon_{i}\left(\boldsymbol{\beta}_{\mathcal{S}}\right) \leq R_{i, \mathcal{S}}\right)+\mathrm{P}\left(\epsilon_{i} \leq R_{i, \mathcal{S}}\right)\right) T_{i l} \boldsymbol{\Pi}\left(\mathbf{X}_{i}\right) \|>c n \lambda_{n}\right) \\
& \leq \sum_{j=1}^{K_{n}} \mathrm{P}\left(\| \sum_{i=1}^{n}\left(I\left(\epsilon_{i}\left(\boldsymbol{\beta}_{\mathcal{S}, j}\right) \leq R_{i, \mathcal{S}}\right)-I\left(\epsilon_{i} \leq R_{i, \mathcal{S}}\right)-\mathrm{P}\left(\epsilon_{i}\left(\boldsymbol{\beta}_{\mathcal{S}, j}\right) \leq R_{i, \mathcal{S}}\right)\right.\right. \\
& \left.\left.+\mathrm{P}\left(\epsilon_{i} \leq R_{i, \mathcal{S}}\right)\right) T_{i l} \boldsymbol{\Pi}\left(\mathbf{X}_{i}\right) \|>c n \lambda_{n} / 2\right) \\
& +\sum_{j=1}^{K_{n}} \mathrm{P}\left(\sup _{\left\|\boldsymbol{\beta}_{\mathcal{S}}-\boldsymbol{\beta}_{\mathcal{S}, j}\right\| \leq C q_{n}^{1 / 2} k_{n} n^{-5 / 2}} \| \sum_{i=1}^{n}\left(I\left(\epsilon_{i}\left(\boldsymbol{\beta}_{\mathcal{S}}\right) \leq R_{i, \mathcal{S}}\right)\right.\right. \\
& \left.-I\left(\epsilon_{i}\left(\boldsymbol{\beta}_{\mathcal{S}, j}\right) \leq R_{i, \mathcal{S}}\right)-\mathrm{P}\left(\epsilon_{i}\left(\boldsymbol{\beta}_{\mathcal{S}}\right) \leq R_{i, \mathcal{S}}\right)+\mathrm{P}\left(\epsilon_{i}\left(\boldsymbol{\beta}_{\mathcal{S}, j}\right) \leq R_{i, \mathcal{S}}\right)\right) \\
& \left.T_{i l} \boldsymbol{\Pi}\left(\mathbf{X}_{i}\right) \|>c n \lambda_{n} / 2\right) \\
& :=I_{n 1}+I_{n 2} \text {. }
\end{aligned}
$$

We let

$a_{i}:=I\left(\epsilon_{i}\left(\boldsymbol{\beta}_{\mathcal{S}, j}\right) \leq R_{i, \mathcal{S}}\right)-I\left(\epsilon_{i} \leq R_{i, \mathcal{S}}\right)-\mathrm{P}\left(\epsilon_{i}\left(\boldsymbol{\beta}_{\mathcal{S}, j}\right) \leq R_{i, \mathcal{S}}\right)+\mathrm{P}\left(\epsilon_{i} \leq R_{i, \mathcal{S}}\right)$. 
Note that $a_{i}$ is bounded with $\mathrm{E} a_{i}=0$ and

$$
\begin{aligned}
\operatorname{Var}\left(a_{i}\right) & =\operatorname{Var}\left(I\left(\epsilon_{i}\left(\boldsymbol{\beta}_{\mathcal{S}, j}\right) \leq R_{i, \mathcal{S}}\right)-I\left(\epsilon_{i} \leq R_{i, \mathcal{S}}\right)\right) \\
& \leq \mathrm{E}\left(I\left(\epsilon_{i}\left(\boldsymbol{\beta}_{\mathcal{S}, j}\right) \leq R_{i, \mathcal{S}}\right)-I\left(\epsilon_{i} \leq R_{i, \mathcal{S}}\right)\right)^{2} \\
& \leq C\left(\left|\mathbf{\Pi}_{\mathcal{S}}\left(\mathbf{X}_{i}, \mathbf{T}_{i}\right)^{\mathrm{T}}\left(\boldsymbol{\beta}_{\mathcal{S}, j}-\boldsymbol{\beta}_{0, \mathcal{S}}\right)\right|+\left|R_{i, \mathcal{S}}\right|\right) .
\end{aligned}
$$

Therefore,

$$
\begin{aligned}
\sum_{i=1}^{n} \operatorname{Var}\left(a_{i}\right) & \leq \sum_{i=1}^{n} C\left(\left|\boldsymbol{\Pi}_{\mathcal{S}}\left(\mathbf{X}_{i}, \mathbf{T}_{i}\right)^{\mathrm{T}}\left(\boldsymbol{\beta}_{\mathcal{S}, j}-\boldsymbol{\beta}_{0, \mathcal{S}}\right)\right|+\left|R_{i, \mathcal{S}}\right|\right) \\
& \leq C\left(q_{n}^{1 / 2} n^{1 / 2} k_{n}+n q_{n} k_{n}^{-r}\right) \\
& \leq C q_{n} n^{1 / 2} k_{n}=o\left(n \lambda_{n} k_{n}^{-1 / 2}\right)
\end{aligned}
$$

Applying Bernstein's inequality, we have

$$
\begin{aligned}
\mathrm{P}\left(\left|\sum_{i=1}^{n} a_{i}\right|>c n \lambda_{n} k_{n}^{-1 / 2}\right) & \leq \exp \left(-\frac{k_{n}^{-1} n^{2} \lambda_{n}^{2} / 4}{c n \lambda_{n} k_{n}^{-1 / 2}+c n \lambda_{n} k_{n}^{-1 / 2}}\right) \\
& =\exp \left(-C n \lambda_{n} k_{n}^{-1 / 2}\right) .
\end{aligned}
$$

Then,

$$
\begin{aligned}
& \mathrm{P}\left(\left\|\sum_{i=1}^{n} a_{i} T_{i l} \boldsymbol{\Pi}\left(\mathbf{X}_{i}\right)\right\|>c n \lambda_{n} / 2\right) \\
= & 1-\mathrm{P}\left(\left\|\sum_{i=1}^{n} a_{i} T_{i l} \boldsymbol{\Pi}\left(\mathbf{X}_{i}\right)\right\| \leq c n \lambda_{n} / 2\right) \\
\leq & 1-\left(\mathrm{P}\left(\left|\sum_{i=1}^{n} a_{i}\right| \leq c n \lambda_{n} k_{n}^{-1 / 2}\right)\right)^{1+d J_{n}} \\
\leq & k_{n} \exp \left(-C n \lambda_{n} k_{n}^{-1 / 2}\right) \\
= & \exp \left(\log \left(k_{n}\right)-C n \lambda_{n} k_{n}^{-1 / 2}\right) .
\end{aligned}
$$

Thus, we have

$$
I_{n 1} \leq K_{n} \exp \left(\log \left(k_{n}\right)-C n \lambda_{n} k_{n}^{-1 / 2}\right)
$$




\section{Chapter 3. Quantile Regression for Additive Coefficient Models}

$$
=\exp \left(C q_{n} k_{n} \log (n)+\log \left(k_{n}\right)-C n \lambda_{n} k_{n}^{-1 / 2}\right) \rightarrow 0,
$$

when $n \lambda_{n} / q_{n} k_{n}^{3 / 2} \log (n) \rightarrow \infty$ and $n \lambda_{n} / k_{n}^{1 / 2} \log \left(k_{n}\right) \rightarrow \infty$. For $I_{n 2}$, we have

$$
\begin{gathered}
\sup _{\left\|\boldsymbol{\beta}_{\mathcal{S}}-\boldsymbol{\beta}_{\mathcal{S}, j}\right\| \leq C q_{n}^{1 / 2} k_{n} n^{-5 / 2}} \| \sum_{i=1}^{n}\left(I\left(\epsilon_{i}\left(\boldsymbol{\beta}_{\mathcal{S}}\right) \leq R_{i, \mathcal{S}}\right)-I\left(\epsilon_{i}\left(\boldsymbol{\beta}_{\mathcal{S}, j}\right) \leq R_{i, \mathcal{S}}\right)\right. \\
\left.-\mathrm{P}\left(\epsilon_{i}\left(\boldsymbol{\beta}_{\mathcal{S}}\right) \leq R_{i, \mathcal{S}}\right)+\mathrm{P}\left(\epsilon_{i}\left(\boldsymbol{\beta}_{\mathcal{S}, j}\right) \leq R_{i, \mathcal{S}}\right)\right) T_{i l} \boldsymbol{\Pi}\left(\mathbf{X}_{i}\right) \| \\
\sup _{\left\|\boldsymbol{\beta}_{\mathcal{S}}-\boldsymbol{\beta}_{\mathcal{S}, j}\right\| \leq C q_{n}^{1 / 2} k_{n} n^{-5 / 2}} \| \sum_{i=1}^{n}\left(I \left(\epsilon_{i}\left(\boldsymbol{\beta}_{\mathcal{S}, j}\right) \leq \boldsymbol{\Pi}_{\mathcal{S}}\left(\mathbf{X}_{i}, \mathbf{T}_{i}\right)^{\mathrm{T}}\left(\boldsymbol{\beta}_{\mathcal{S}}-\boldsymbol{\beta}_{\mathcal{S}, j}\right)\right.\right. \\
\left.+R_{i, \mathcal{S}}\right)-I\left(\epsilon_{i}\left(\boldsymbol{\beta}_{\mathcal{S}, j}\right) \leq R_{i, \mathcal{S}}\right)-\mathrm{P}\left(\epsilon_{i}\left(\boldsymbol{\beta}_{\mathcal{S}, j}\right) \leq \boldsymbol{\Pi}_{\mathcal{S}}\left(\mathbf{X}_{i}, \mathbf{T}_{i}\right)^{\mathrm{T}}\left(\boldsymbol{\beta}_{\mathcal{S}}-\boldsymbol{\beta}_{\mathcal{S}, j}\right)\right. \\
\left.\left.+R_{i, \mathcal{S}}\right)+\mathrm{P}\left(\epsilon_{i}\left(\boldsymbol{\beta}_{\mathcal{S}, j}\right) \leq R_{i, \mathcal{S}}\right)\right) T_{i l} \boldsymbol{\Pi}\left(\mathbf{X}_{i}\right) \| \\
\quad \sup _{\left\|\boldsymbol{\beta}_{\mathcal{S}}-\boldsymbol{\beta}_{\mathcal{S}, j}\right\| \leq C q_{n}^{1 / 2} k_{n} n^{-5 / 2}} \| \sum_{i=1}^{n}\left(I\left(\epsilon_{i}\left(\boldsymbol{\beta}_{\mathcal{S}, j}\right) \leq C q_{n} k_{n} n^{-5 / 2}+R_{i, \mathcal{S}}\right)\right. \\
\quad-I\left(\epsilon_{i}\left(\boldsymbol{\beta}_{\mathcal{S}, j}\right) \leq R_{i, \mathcal{S}}\right)-\mathrm{P}\left(\epsilon_{i}\left(\boldsymbol{\beta}_{\mathcal{S}, j}\right) \leq-C q_{n} k_{n} n^{-5 / 2}+R_{i, \mathcal{S}}\right) \\
\left.+\mathrm{P}\left(\epsilon_{i}\left(\boldsymbol{\beta}_{\mathcal{S}, j}\right) \leq R_{i, \mathcal{S}}\right)\right) T_{i l} \boldsymbol{\Pi}\left(\mathbf{X}_{i}\right) \| \\
\quad \sup _{\left\|\boldsymbol{\beta}_{\mathcal{S}}-\boldsymbol{\beta}_{\mathcal{S}, j}\right\| \leq C q_{n}^{1 / 2} k_{n} n^{-5 / 2}} \| \sum_{i=1}^{n}\left(I\left(\epsilon_{i}\left(\boldsymbol{\beta}_{\mathcal{S}, j}\right) \leq C q_{n} k_{n} n^{-5 / 2}+R_{i, \mathcal{S}}\right)\right. \\
\quad-I\left(\epsilon_{i}\left(\boldsymbol{\beta}_{\mathcal{S}, j}\right) \leq R_{i, \mathcal{S}}\right)-\mathrm{P}\left(\epsilon_{i}\left(\boldsymbol{\beta}_{\mathcal{S}, j}\right) \leq C q_{n} k_{n} n^{-5 / 2}+R_{i, \mathcal{S}}\right) \\
\left.+\mathrm{P}\left(\epsilon_{i}\left(\boldsymbol{\beta}_{\mathcal{S}, j}\right) \leq R_{i, \mathcal{S}}\right)\right) T_{i l} \boldsymbol{\Pi}\left(\mathbf{X}_{i}\right)\|+\| \sum_{i=1}^{n}\left(\mathrm { P } \left(\epsilon_{i}\left(\boldsymbol{\beta}_{\mathcal{S}, j}\right) \leq C q_{n} k_{n} n^{-5 / 2}\right.\right. \\
\left.\left.+R_{i, \mathcal{S}}\right)-\mathrm{P}\left(\epsilon_{i}\left(\boldsymbol{\beta}_{\mathcal{S}, j}\right) \leq-C q_{n} k_{n} n^{-5 / 2}+R_{i, \mathcal{S}}\right)\right) T_{i l} \boldsymbol{\Pi}\left(\mathbf{X}_{i}\right) \|
\end{gathered}
$$

First, we consider the second term in 3.11.

$$
\begin{gathered}
\| \sum_{i=1}^{n}\left(\mathrm{P}\left(\epsilon_{i}\left(\boldsymbol{\beta}_{\mathcal{S}, j}\right) \leq C q_{n} k_{n} n^{-5 / 2}+R_{i, \mathcal{S}}\right)\right. \\
\left.-\mathrm{P}\left(\epsilon_{i}\left(\boldsymbol{\beta}_{\mathcal{S}, j}\right) \leq-C q_{n} k_{n} n^{-5 / 2}+R_{i, \mathcal{S}}\right)\right) T_{i l} \boldsymbol{\Pi}\left(\mathbf{X}_{i}\right) \| \\
\leq C k_{n}^{1 / 2} \mid \sum_{i=1}^{n}\left(\mathrm{P}\left(\epsilon_{i}\left(\boldsymbol{\beta}_{\mathcal{S}, j}\right) \leq C q_{n} k_{n} n^{-5 / 2}+R_{i, \mathcal{S}}\right)\right. \\
\left.-\mathrm{P}\left(\epsilon_{i}\left(\boldsymbol{\beta}_{\mathcal{S}, j}\right) \leq-C q_{n} k_{n} n^{-5 / 2}+R_{i, \mathcal{S}}\right)\right) \mid
\end{gathered}
$$




$$
\begin{aligned}
& =C k_{n}^{1 / 2}\left|\sum_{i=1}^{n}\left(F_{i}\left(C q_{n} k_{n} n^{-5 / 2}+R_{i, \mathcal{S}}\right)-F_{i}\left(-C q_{n} k_{n} n^{-5 / 2}+R_{i, \mathcal{S}}\right)\right)\right| \\
& \leq C q_{n} k_{n}^{3 / 2} n^{-3 / 2}=o\left(n \lambda_{n}\right) .
\end{aligned}
$$

Then we consider the first term in (3.11). Let

$$
\begin{aligned}
b_{i}:= & I\left(\epsilon_{i}\left(\boldsymbol{\beta}_{\mathcal{S}, j}\right) \leq C q_{n} k_{n} n^{-5 / 2}+R_{i, \mathcal{S}}\right)-I\left(\epsilon_{i}\left(\boldsymbol{\beta}_{\mathcal{S}, j}\right) \leq R_{i, \mathcal{S}}\right) \\
& -\mathrm{P}\left(\epsilon_{i}\left(\boldsymbol{\beta}_{\mathcal{S}, j}\right) \leq C q_{n} k_{n} n^{-5 / 2}+R_{i, \mathcal{S}}\right)+\mathrm{P}\left(\epsilon_{i}\left(\boldsymbol{\beta}_{\mathcal{S}, j}\right) \leq R_{i, \mathcal{S}}\right) .
\end{aligned}
$$

Note that $b_{i}$ is bounded with $\mathrm{E} b_{i}=0$ and

$$
\begin{aligned}
\operatorname{Var}\left(b_{i}\right) & =\operatorname{Var}\left(I\left(\epsilon_{i}\left(\boldsymbol{\beta}_{\mathcal{S}, j}\right) \leq C q_{n} k_{n} n^{-5 / 2}+R_{i, \mathcal{S}}\right)-I\left(\epsilon_{i}\left(\boldsymbol{\beta}_{\mathcal{S}, j}\right) \leq R_{i, \mathcal{S}}\right)\right) \\
& \leq \mathrm{E}\left(I\left(\epsilon_{i}\left(\boldsymbol{\beta}_{\mathcal{S}, j}\right) \leq C q_{n} k_{n} n^{-5 / 2}+R_{i, \mathcal{S}}\right)-I\left(\epsilon_{i}\left(\boldsymbol{\beta}_{\mathcal{S}, j}\right) \leq R_{i, \mathcal{S}}\right)\right)^{2} \\
& \leq C q_{n} k_{n}^{-r} .
\end{aligned}
$$

Thus,

$$
\sum_{i=1}^{n} \operatorname{Var}\left(b_{i}\right) \leq C n q_{n} k_{n}^{-r}=o\left(n \lambda_{n} k_{n}^{-1 / 2}\right) .
$$

Applying Bernstein's inequality, we have

$$
\begin{aligned}
\mathrm{P}\left(\left|\sum_{i=1}^{n} b_{i}\right|>n \lambda_{n} k_{n}^{-1 / 2} / 4\right) & \leq \exp \left(-\frac{k_{n}^{-1} n^{2} \lambda_{n}^{2} / 16}{c n \lambda_{n} k_{n}^{-1 / 2}+C n \lambda_{n} k_{n}^{-1 / 2}}\right) \\
& \leq \exp \left(-C n \lambda_{n} k_{n}^{-1 / 2}\right) .
\end{aligned}
$$

Therefore, we have

$$
\begin{aligned}
\mathrm{P}\left(\left\|\sum_{i=1}^{n} b_{i} T_{i l} \boldsymbol{\Pi}\left(\mathbf{X}_{i}\right)\right\|>n \lambda_{n} / 4\right) & \leq k_{n} \exp \left(-C n \lambda_{n} k_{n}^{-1 / 2}\right) \\
& =\exp \left(\log \left(k_{n}\right)-C n \lambda_{n} k_{n}^{-1 / 2}\right) .
\end{aligned}
$$

Thus,

$$
I_{n 2} \leq K_{n} \exp \left(\log \left(k_{n}\right)-C n \lambda_{n} k_{n}^{-1 / 2}\right)
$$




\section{Chapter 3. Quantile Regression for Additive Coefficient Models}

$$
\begin{aligned}
& =\exp \left(\log K_{n}+\log \left(k_{n}\right)-C n \lambda_{n} k_{n}^{-1 / 2}\right) \\
& \leq \exp \left(c q_{n} k_{n} \log (n)+\log \left(k_{n}\right)-C n \lambda_{n} k_{n}^{-1 / 2}\right) \rightarrow 0,
\end{aligned}
$$

when $n \lambda_{n} / q_{n} k_{n}^{3 / 2} \log (n) \rightarrow \infty$ and $n \lambda_{n} / k_{n}^{1 / 2} \log \left(k_{n}\right) \rightarrow \infty$. Thus, (3.11) is bounded by

$$
\sum_{l \in \mathcal{S}^{c}}\left(I_{n 1}+I_{n 2}\right) \leq p_{n} \exp \left(c q_{n} k_{n} \log n+\log \left(k_{n}\right)-C n \lambda_{n} k_{n}^{-1 / 2}\right) \rightarrow 0
$$

when $n \lambda_{n} /\left(k_{n}^{1 / 2} \log \left(p_{n}\right)\right) \rightarrow \infty$.

Lemma 3.4. Under the Conditions (C1)-(C7), if $\lambda_{n}=o\left(n^{-\left(1-c_{4}\right) / 2} k_{n}^{1 / 2}\right)$, $q_{n} k_{n}^{3 / 2} n^{-1 / 2}=o\left(\lambda_{n}\right)$ and $n \lambda_{n} /\left(k_{n}^{1 / 2} \log \left(p_{n}\right)\right) \rightarrow \infty$, for the oracle estimator $\hat{\boldsymbol{\beta}}$, we have, with probability approaching 1,

(1) $\left\|\boldsymbol{\nu}_{l}(\hat{\boldsymbol{\beta}})\right\|=0$, for $l \in \mathcal{S}$,

(2) $\left\|\hat{\boldsymbol{\beta}}_{l}\right\|>(a+1 / 2) \lambda_{n}$, for $l \in \mathcal{S}$,

(3) $\left\|\boldsymbol{\nu}_{l}(\hat{\boldsymbol{\beta}})\right\| \leq c n \lambda_{n}$, for $l \in \mathcal{S}^{c}$, and any $c>0$.

Proof. (1) obviously holds by the definition of oracle estimator $\hat{\boldsymbol{\beta}}=\left(\hat{\boldsymbol{\beta}}_{\mathcal{S}}^{\mathrm{T}}, \mathbf{0}^{\mathrm{T}}\right)^{\mathrm{T}}$.

(2) From (C7), we have $\min _{l \in \mathcal{S}}\left\|\boldsymbol{\beta}_{0, l}\right\| \geq c_{5} k_{n}^{1 / 2} n^{-\left(1-c_{4}\right) / 2}$. By Theorem 3.1, we have

$$
\max _{l \in \mathcal{S}}\left\|\hat{\boldsymbol{\beta}}_{l}-\boldsymbol{\beta}_{0, l}\right\|=O_{p}\left(k_{n} n^{-1 / 2}\right)=o_{p}\left(k_{n}^{1 / 2} n^{-\left(1-c_{4}\right) / 2}\right) .
$$

Thus, for $l \in \mathcal{S}$,

$$
\left\|\hat{\boldsymbol{\beta}}_{l}\right\| \geq\left\|\boldsymbol{\beta}_{0, l}\right\|-\left\|\hat{\boldsymbol{\beta}}_{l}-\boldsymbol{\beta}_{0, l}\right\| \geq c_{2} n^{-\left(1-c_{4}\right) / 2} k_{n}^{1 / 2} \geq(a+1 / 2) \lambda_{n}
$$

where $c_{2}$ is a positive constant and the above holds when $\lambda_{n}=$ $o\left(n^{-\left(1-c_{4}\right) / 2} k_{n}^{1 / 2}\right)$. 
(3) From the definition of $\boldsymbol{\nu}_{l}(\boldsymbol{\beta})$ in $(3.6)$, we have

$$
\begin{aligned}
\boldsymbol{\nu}_{l}(\hat{\boldsymbol{\beta}})= & \sum_{i=1}^{n}\left(-\tau I\left(Y_{i}-\boldsymbol{\Pi}_{\mathcal{S}}\left(\mathbf{X}_{i}, \mathbf{T}_{i}\right)^{\mathrm{T}} \hat{\boldsymbol{\beta}}_{\mathcal{S}}>0\right)+(1-\tau) I\left(Y_{i}\right.\right. \\
& \left.\left.-\boldsymbol{\Pi}_{\mathcal{S}}\left(\mathbf{X}_{i}, \mathbf{T}_{i}\right)^{\mathrm{T}} \hat{\boldsymbol{\beta}}_{\mathcal{S}}<0\right)\right) T_{i l} \boldsymbol{\Pi}\left(\mathbf{X}_{i}\right)-\sum_{i=1}^{n} a_{i l}^{*} T_{i l} \boldsymbol{\Pi}\left(\mathbf{X}_{i}\right)
\end{aligned}
$$

where $l \in \mathcal{S}^{c}$, and $a_{i l}^{*}$ satisfied the condition in (3.6). Let $\mathcal{F}:=\{i$ : $\left.Y_{i}-\boldsymbol{\Pi}_{\mathcal{S}}\left(\mathbf{X}_{i}, \mathbf{T}_{i}\right)^{\mathrm{T}} \hat{\boldsymbol{\beta}}_{\mathcal{S}}=0\right\}$. Then we need to show

$$
\begin{array}{r}
\|\left(\sum_{i=1}^{n}\left(I\left(Y_{i}-\boldsymbol{\Pi}_{\mathcal{S}}\left(\mathbf{X}_{i}, \mathbf{T}_{i}\right)^{\mathrm{T}} \hat{\boldsymbol{\beta}}_{\mathcal{S}} \leq 0\right)-\tau\right)-\sum_{i \in \mathcal{F}}(1-\tau)\right) T_{i l} \boldsymbol{\Pi}\left(\mathbf{X}_{i}\right) \\
-\sum_{i \in \mathcal{F}} a_{i l}^{*} T_{i l} \boldsymbol{\Pi}\left(\mathbf{X}_{i}\right) \| \leq n \lambda_{n} .
\end{array}
$$

From Koenker (2005), we have $|\mathcal{F}|=O_{p}\left(q_{n} k_{n}\right)$. Thus, we have

$$
\left\|\sum_{i \in \mathcal{F}} a_{i l}^{*} T_{i l} \boldsymbol{\Pi}\left(\mathbf{X}_{i}\right)\right\|=O_{p}\left(q_{n}^{1 / 2} k_{n}^{1 / 2}\right)=o_{p}\left(n \lambda_{n}\right)
$$

and

$$
\left\|\sum_{i \in \mathcal{F}}(1-\tau) T_{i l} \boldsymbol{\Pi}\left(\mathbf{X}_{i}\right)\right\|=O_{p}\left(q_{n}^{1 / 2} k_{n}^{1 / 2}\right)=o_{p}\left(n \lambda_{n}\right)
$$

Then, it is sufficient to show

$$
\mathrm{P}\left(\max _{l \in \mathcal{S}^{c}}\left\|\sum_{i=1}^{n}\left(I\left(Y_{i}-\mathbf{\Pi}_{\mathcal{S}}\left(\mathbf{X}_{i}, \mathbf{T}_{i}\right)^{\mathrm{T}} \hat{\boldsymbol{\beta}}_{\mathcal{S}} \leq 0\right)-\tau\right) T_{i l} \boldsymbol{\Pi}\left(\mathbf{X}_{i}\right)\right\|>c n \lambda_{n}\right)
$$

turns to 0 . Note that

$$
\begin{gathered}
\mathrm{P}\left(\max _{l \in \mathcal{S}^{c}}\left\|\sum_{i=1}^{n}\left(I\left(Y_{i}-\boldsymbol{\Pi}_{\mathcal{S}}\left(\mathbf{X}_{i}, \mathbf{T}_{i}\right)^{\mathrm{T}} \hat{\boldsymbol{\beta}}_{\mathcal{S}} \leq 0\right)-\tau\right) T_{i l} \boldsymbol{\Pi}\left(\mathbf{X}_{i}\right)\right\|\right. \\
\left.>c n \lambda_{n}\right) \\
\leq \mathrm{P}\left(\max _{l \in \mathcal{S}^{c}}\left\|\sum_{i=1}^{n}\left(I\left(Y_{i}-\boldsymbol{\Pi}_{\mathcal{S}}\left(\mathbf{X}_{i}, \mathbf{T}_{i}\right)^{\mathrm{T}} \boldsymbol{\beta}_{0, \mathcal{S}} \leq 0\right)-\tau\right) T_{i l} \boldsymbol{\Pi}\left(\mathbf{X}_{i}\right)\right\|\right.
\end{gathered}
$$




$$
\begin{gathered}
\left.>c n \lambda_{n} / 2\right)+\mathrm{P}\left(\max _{l \in \mathcal{S}^{c}} \| \sum_{i=1}^{n}\left(I\left(Y_{i}-\boldsymbol{\Pi}_{\mathcal{S}}\left(\mathbf{X}_{i}, \mathbf{T}_{i}\right)^{\mathrm{T}} \hat{\boldsymbol{\beta}}_{\mathcal{S}} \leq 0\right)\right.\right. \\
\left.\left.-I\left(Y_{i}-\boldsymbol{\Pi}_{\mathcal{S}}\left(\mathbf{X}_{i}, \mathbf{T}_{i}\right)^{\mathrm{T}} \boldsymbol{\beta}_{0, \mathcal{S}} \leq 0\right)\right) T_{i l} \boldsymbol{\Pi}\left(\mathbf{X}_{i}\right) \|>c n \lambda_{n} / 2\right) \\
\leq \mathrm{P}\left(\max _{l \in \mathcal{S}^{c}}\left\|\sum_{i=1}^{n}\left(I\left(Y_{i}-\boldsymbol{\Pi}_{\mathcal{S}}\left(\mathbf{X}_{i}, \mathbf{T}_{i}\right)^{\mathrm{T}} \boldsymbol{\beta}_{0, \mathcal{S}} \leq 0\right)-\tau\right) T_{i l} \boldsymbol{\Pi}\left(\mathbf{X}_{i}\right)\right\|\right. \\
\left.>c n \lambda_{n} / 2\right)+\mathrm{P}\left(\operatorname { m a x } _ { l \in \mathcal { S } ^ { c } } \| \sum _ { \boldsymbol { \beta } _ { \mathcal { S } } - \boldsymbol { \beta } _ { 0 , \mathcal { S } } \| \leq C q _ { n } ^ { 1 / 2 } k _ { n } n ^ { - 1 / 2 } } ^ { n } \| \sum _ { i = 1 } \left(I \left(Y_{i}\right.\right.\right. \\
\left.\left.-\boldsymbol{\Pi}_{\mathcal{S}}\left(\mathbf{X}_{i}, \mathbf{T}_{i}\right)^{\mathrm{T}} \boldsymbol{\beta}_{\mathcal{S}} \leq 0\right)-I\left(Y_{i}-\boldsymbol{\Pi}_{\mathcal{S}}\left(\mathbf{X}_{i}, \mathbf{T}_{i}\right)^{\mathrm{T}} \boldsymbol{\beta}_{0, \mathcal{S}} \leq 0\right)\right) \\
\left.T_{i l} \boldsymbol{\Pi}\left(\mathbf{X}_{i}\right) \|>c n \lambda_{n} / 2\right) .
\end{gathered}
$$

Considering the first term in (3.12), we need to show

$\mathrm{P}\left(\max _{l \in \mathcal{S}^{c}}\left\|\sum_{i=1}^{n}\left(I\left(Y_{i}-\boldsymbol{\Pi}_{\mathcal{S}}\left(\mathbf{X}_{i}, \mathbf{T}_{i}\right)^{\mathrm{T}} \boldsymbol{\beta}_{0, \mathcal{S}} \leq 0\right)-\tau\right) T_{i l} \boldsymbol{\Pi}\left(\mathbf{X}_{i}\right)\right\|>c n \lambda_{n} / 2\right)$

turns to 0. Using Hoeffding's inequality, we have

$$
\begin{aligned}
& \mathrm{P}\left(\left|\sum_{i=1}^{n}\left(I\left(Y_{i}-\boldsymbol{\Pi}_{\mathcal{S}}\left(\mathbf{X}_{i}, \mathbf{T}_{i}\right)^{\mathrm{T}} \boldsymbol{\beta}_{0, \mathcal{S}} \leq 0\right)-\tau\right) T_{i l}\right|>c n \lambda_{n} k_{n}^{-1 / 2} / 2\right) \\
\leq & 2 \exp \left(-C n \lambda_{n}^{2} k_{n}^{-1}\right),
\end{aligned}
$$

for any $l \in \mathcal{S}^{c}$. Thus, we have

$$
\begin{aligned}
& \mathrm{P}\left(\left\|\sum_{i=1}^{n}\left(I\left(Y_{i}-\boldsymbol{\Pi}_{\mathcal{S}}\left(\mathbf{X}_{i}, \mathbf{T}_{i}\right)^{\mathrm{T}} \boldsymbol{\beta}_{0, \mathcal{S}} \leq 0\right)-\tau\right) T_{i l} \boldsymbol{\Pi}\left(\mathbf{X}_{i}\right)\right\|>c n \lambda_{n} / 2\right) \\
= & 1-\mathrm{P}\left(\left\|\sum_{i=1}^{n}\left(I\left(Y_{i}-\boldsymbol{\Pi}_{\mathcal{S}}\left(\mathbf{X}_{i}, \mathbf{T}_{i}\right)^{\mathrm{T}} \boldsymbol{\beta}_{0, \mathcal{S}} \leq 0\right)-\tau\right) T_{i l} \boldsymbol{\Pi}\left(\mathbf{X}_{i}\right)\right\| \leq c n \lambda_{n} / 2\right) \\
\leq & 1-\left(\mathrm { P } \left(\left|\sum_{i=1}^{n}\left(I\left(Y_{i}-\boldsymbol{\Pi}_{\mathcal{S}}\left(\mathbf{X}_{i}, \mathbf{T}_{i}\right)^{\mathrm{T}} \boldsymbol{\beta}_{0, \mathcal{S}} \leq 0\right)-\tau\right) T_{i l}\right|\right.\right. \\
& \left.\left.\leq c n \lambda_{n} k_{n}^{-1 / 2} / 2\right)\right)^{1+d J_{n}} \\
\leq & 2 C k_{n} \exp \left(-C n \lambda_{n}^{2} k_{n}^{-1}\right) \\
= & 2 C \exp \left(\log \left(k_{n}\right)-C n \lambda_{n}^{2} k_{n}^{-1}\right) .
\end{aligned}
$$


Hence, using the union bound, we have

$$
\begin{aligned}
& \mathrm{P}\left(\max _{l \in \mathcal{S}^{c}}\left\|\sum_{i=1}^{n}\left(I\left(Y_{i}-\boldsymbol{\Pi}_{\mathcal{S}}\left(\mathbf{X}_{i}, \mathbf{T}_{i}\right)^{\mathrm{T}} \boldsymbol{\beta}_{0, \mathcal{S}} \leq 0\right)-\tau\right) T_{i l} \boldsymbol{\Pi}\left(\mathbf{X}_{i}\right)\right\|\right. \\
&\left.>c n \lambda_{n} / 2\right) \\
& \leq 2 p_{n} \exp \left(\log \left(k_{n}\right)-C n \lambda_{n}^{2} k_{n}^{-1}\right) \\
&=2 \exp \left(\log \left(p_{n}\right)+\log \left(k_{n}\right)-C n \lambda_{n}^{2} k_{n}^{-1}\right) .
\end{aligned}
$$

Thus, when $n \lambda_{n}^{2} / k_{n} \log \left(k_{n}\right) \rightarrow \infty$ and $\log \left(p_{n}\right)=o_{p}\left(n \lambda_{n}^{2} k_{n}^{-1}\right)$, the first term in 3.12 turns to 0 .

Considering the second term in 3.12 , we have

$$
\begin{aligned}
& \mathrm{P}\left(\max _{l \in \mathcal{S}^{c}} \sup _{\left\|\boldsymbol{\beta}_{\mathcal{S}}-\boldsymbol{\beta}_{0, \mathcal{S}}\right\| \leq C q_{n}^{1 / 2} k_{n} n^{-1 / 2}} \| \sum_{i=1}^{n}\left(I \left(Y_{i}-\boldsymbol{\Pi}_{\mathcal{S}}\left(\mathbf{X}_{i}, \mathbf{T}_{i}\right)^{\mathrm{T}} \boldsymbol{\beta}_{\mathcal{S}}\right.\right.\right. \\
& \left.\left.\leq 0)-I\left(Y_{i}-\boldsymbol{\Pi}_{\mathcal{S}}\left(\mathbf{X}_{i}, \mathbf{T}_{i}\right)^{\mathrm{T}} \boldsymbol{\beta}_{0, \mathcal{S}} \leq 0\right)\right) T_{i l} \boldsymbol{\Pi}\left(\mathbf{X}_{i}\right) \|>c n \lambda_{n} / 2\right) \\
& \leq \mathrm{P}\left(\max _{l \in \mathcal{S}^{c}} \sup _{\left\|\boldsymbol{\beta}_{\mathcal{S}}-\boldsymbol{\beta}_{0, \mathcal{S}}\right\| \leq C q_{n}^{1 / 2} k_{n} n^{-1 / 2}} \| \sum_{i=1}^{n}\left(I \left(Y_{i}-\boldsymbol{\Pi}_{\mathcal{S}}\left(\mathbf{X}_{i}, \mathbf{T}_{i}\right)^{\mathrm{T}} \boldsymbol{\beta}_{\mathcal{S}}\right.\right.\right. \\
& \leq 0)-I\left(Y_{i}-\boldsymbol{\Pi}_{\mathcal{S}}\left(\mathbf{X}_{i}, \mathbf{T}_{i}\right)^{\mathrm{T}} \boldsymbol{\beta}_{0, \mathcal{S}} \leq 0\right)-\mathrm{P}\left(Y_{i}-\boldsymbol{\Pi}_{\mathcal{S}}\left(\mathbf{X}_{i}, \mathbf{T}_{i}\right)^{\mathrm{T}} \boldsymbol{\beta}_{\mathcal{S}}\right. \\
& \left.\left.\leq 0)+\mathrm{P}\left(Y_{i}-\boldsymbol{\Pi}_{\mathcal{S}}\left(\mathbf{X}_{i}, \mathbf{T}_{i}\right)^{\mathrm{T}} \boldsymbol{\beta}_{0, \mathcal{S}} \leq 0\right)\right) T_{i l} \boldsymbol{\Pi}\left(\mathbf{X}_{i}\right) \|>c n \lambda_{n} / 4\right) \\
& +\mathrm{P}\left(\max _{l \in \mathcal{S}^{c}} \sup _{\left\|\boldsymbol{\beta}_{\mathcal{S}}-\boldsymbol{\beta}_{0, \mathcal{S}}\right\| \leq C q_{n}^{1 / 2} k_{n} n^{-1 / 2}} \| \sum_{i=1}^{n}\left(\mathrm{P}\left(Y_{i}-\boldsymbol{\Pi}_{\mathcal{S}}\left(\mathbf{X}_{i}, \mathbf{T}_{i}\right)^{\mathrm{T}} \boldsymbol{\beta}_{\mathcal{S}} \leq 0\right)\right.\right. \\
& \left.\left.-\mathrm{P}\left(Y_{i}-\boldsymbol{\Pi}_{\mathcal{S}}\left(\mathbf{X}_{i}, \mathbf{T}_{i}\right)^{\mathrm{T}} \boldsymbol{\beta}_{0, \mathcal{S}} \leq 0\right)\right) T_{i l} \boldsymbol{\Pi}\left(\mathbf{X}_{i}\right) \|>c n \lambda_{n} / 4\right) \text {. }
\end{aligned}
$$

From Lemma 3.3, the first term in (3.13) converges to zero. For the second term in $(3.13)$, we have

$$
\begin{gathered}
\max _{l \in \mathcal{S}^{c}} \sup _{\left\|\boldsymbol{\beta}_{\mathcal{S}}-\boldsymbol{\beta}_{0, \mathcal{S}}\right\| \leq C q_{n}^{1 / 2} k_{n} n^{-1 / 2}} \| \sum_{i=1}^{n}\left(\mathrm{P}\left(Y_{i}-\mathbf{\Pi}_{\mathcal{S}}\left(\mathbf{X}_{i}, \mathbf{T}_{i}\right)^{\mathrm{T}} \boldsymbol{\beta}_{\mathcal{S}} \leq 0\right)\right. \\
\\
\left.-\mathrm{P}\left(Y_{i}-\mathbf{\Pi}_{\mathcal{S}}\left(\mathbf{X}_{i}, \mathbf{T}_{i}\right)^{\mathrm{T}} \boldsymbol{\beta}_{0, \mathcal{S}} \leq 0\right)\right) T_{i l} \boldsymbol{\Pi}\left(\mathbf{X}_{i}\right) \|
\end{gathered}
$$




$$
\begin{aligned}
& \leq C k_{n}^{1 / 2} \sup _{\left\|\boldsymbol{\beta}_{\mathcal{S}}-\boldsymbol{\beta}_{0, \mathcal{S}}\right\| \leq C q_{n}^{1 / 2} k_{n} n^{-1 / 2}} \sum_{i=1}^{n} \mid \mathrm{P}\left(Y_{i}-\boldsymbol{\Pi}_{\mathcal{S}}\left(\mathbf{X}_{i}, \mathbf{T}_{i}\right)^{\mathrm{T}} \boldsymbol{\beta}_{\mathcal{S}} \leq 0\right) \\
& -\mathrm{P}\left(Y_{i}-\boldsymbol{\Pi}_{\mathcal{S}}\left(\mathbf{X}_{i}, \mathbf{T}_{i}\right)^{\mathrm{T}} \boldsymbol{\beta}_{0, \mathcal{S}} \leq 0\right) \mid \\
& \leq C k_{n}^{1 / 2} \sup _{\left\|\boldsymbol{\beta}_{\mathcal{S}}-\boldsymbol{\beta}_{0, \mathcal{S}}\right\| \leq C q_{n}^{1 / 2} k_{n} n^{-1 / 2}} \sum_{i=1}^{n} \mid F_{i}\left(R_{i, \mathcal{S}}+\boldsymbol{\Pi}_{\mathcal{S}}\left(\mathbf{X}_{i}, \mathbf{T}_{i}\right)^{\mathrm{T}}\left(\boldsymbol{\beta}_{\mathcal{S}}\right.\right. \\
& \left.\left.-\boldsymbol{\beta}_{0, \mathcal{S}}\right)\right)-F_{i}\left(R_{i, \mathcal{S}}\right)\left|\sum_{\left\|\boldsymbol{\beta}_{\mathcal{S}}-\boldsymbol{\beta}_{0, \mathcal{S}}\right\| \leq C q_{n}^{1 / 2} k_{n} n^{-1 / 2}} \sum_{i=1}^{n}\right| \boldsymbol{\Pi}_{\mathcal{S}}\left(\mathbf{X}_{i}, \mathbf{T}_{i}\right)^{\mathrm{T}}\left(\boldsymbol{\beta}_{\mathcal{S}}-\boldsymbol{\beta}_{0, \mathcal{S}}\right) \\
& +O\left(\left(\boldsymbol{\Pi}_{\mathcal{S}}\left(\mathbf{X}_{i}, \mathbf{T}_{i}\right)^{\mathrm{T}}\left(\boldsymbol{\beta}_{\mathcal{S}}-\boldsymbol{\beta}_{0, \mathcal{S}}\right)\right)^{2}\right) \mid \\
& \leq C k_{n}^{1 / 2} \sup _{n}+C q_{n}^{3 / 2} n^{1 / 2}=o\left(n \lambda_{n}\right) .
\end{aligned}
$$

This completes the proof.

Finally, from Tao and An (1997), we have the following result.

Lemma 3.5. (Sufficient Local Optimality) Consider the function $G_{n}(\boldsymbol{\beta})-$ $H_{n}(\boldsymbol{\beta})$ where both $G_{n}$ and $H_{n}$ are convex with subdifferential functions $\partial G_{n}(\boldsymbol{\beta})$ and $\partial H_{n}(\boldsymbol{\beta})$. Let $\tilde{\boldsymbol{\beta}}$ be a point that has neighborhood $U$ such that $\partial G_{n}(\tilde{\boldsymbol{\beta}}) \cap \partial H_{n}(\boldsymbol{\beta}) \neq \emptyset, \forall \boldsymbol{\beta} \in U \cap \operatorname{dom}\left(G_{n}\right)$. Then $\tilde{\boldsymbol{\beta}}$ is a local minimizer of $G_{n}(\boldsymbol{\beta})-H_{n}(\boldsymbol{\beta})$.

\section{Proof of Theorem 3.1}

Note that

$$
\begin{aligned}
& \frac{1}{n} \sum_{l \in \mathcal{S}} \sum_{i=1}^{n}\left(\hat{\alpha}_{l}\left(\mathbf{X}_{i}\right)-\alpha_{0, l}\left(\mathbf{X}_{i}\right)\right)^{2} \\
= & \frac{1}{n} \sum_{l \in \mathcal{S}} \sum_{i=1}^{n}\left(\boldsymbol{\Pi}\left(\mathbf{X}_{i}\right)^{\mathrm{T}}\left(\hat{\boldsymbol{\beta}}_{l}-\boldsymbol{\beta}_{0, l}\right)+r_{i l}\right)^{2}
\end{aligned}
$$




$$
\begin{aligned}
& \leq 2 q_{n} n^{-1} \sum_{i=1}^{n}\left(\left(\boldsymbol{\Pi}\left(\mathbf{X}_{i}\right)^{\mathrm{T}}\left(\hat{\boldsymbol{\beta}}_{l}-\boldsymbol{\beta}_{0, l}\right)\right)^{2}+C M_{1}^{2} q_{n} k_{n}^{-2 r}\right) \\
& \leq C q_{n} k_{n}^{-1}\left\|\hat{\boldsymbol{\beta}}_{l}-\boldsymbol{\beta}_{0, l}\right\|^{2}+C M_{1}^{2} q_{n} k_{n}^{-2 r} .
\end{aligned}
$$

In order to get the rate of convergence, it sufficient to show that $\| \hat{\boldsymbol{\beta}}_{\mathcal{S}}-$ $\boldsymbol{\beta}_{0, \mathcal{S}} \|=O_{p}\left(q_{n}^{1 / 2} k_{n} n^{-1 / 2}\right)$. Thus we need to show $\left\|\hat{\boldsymbol{\beta}}_{\mathcal{S}}^{*}-\boldsymbol{\beta}_{0, \mathcal{S}}^{*}\right\|=O_{p}\left(q_{n}^{1 / 2} k_{n}^{1 / 2}\right)$.

From Lemmas 3.1 and 3.2 , we have with probability approaching 1,

$$
\begin{aligned}
& \inf _{\left\|\boldsymbol{\beta}_{\mathcal{S}}^{*}\right\|=L q_{n}^{1 / 2} k_{n}^{1 / 2}} q_{n}^{-1} k_{n}^{-1} \sum_{i=1}^{n}\left[\rho_{\tau}\left(\epsilon_{i}-\mathbf{Z}_{i, \mathcal{S}}^{\mathrm{T}} \boldsymbol{\beta}_{\mathcal{S}}^{*}-R_{i, \mathcal{S}}\right)-\rho_{\tau}\left(\epsilon_{i}-R_{i, \mathcal{S}}\right)\right] \\
& \geq \inf _{\left\|\boldsymbol{\beta}_{\mathcal{S}}^{*}\right\|=L q_{n}^{1 / 2} k_{n}^{1 / 2}}\left\{q_{n}^{-1} k_{n}^{-1} \sum_{i=1}^{n}\left[\mathrm{E}\left(\rho_{\tau}\left(\epsilon_{i}-\mathbf{Z}_{i, \mathcal{S}}^{\mathrm{T}} \boldsymbol{\beta}_{\mathcal{S}}^{*}-R_{i, \mathcal{S}}\right)-\rho_{\tau}\left(\epsilon_{i}-R_{i, \mathcal{S}}\right)\right)\right]\right. \\
& \left.\quad-q_{n}^{-1} k_{n}^{-1} \sum_{i=1}^{n} \mathbf{Z}_{i, \mathcal{S}}^{\mathrm{T}} \boldsymbol{\beta}_{\mathcal{S}}^{*}\left(\tau-I\left(\epsilon_{i} \leq 0\right)\right)\right\}-\epsilon \\
& >1-\epsilon .
\end{aligned}
$$

Setting $\epsilon=\frac{1}{2}$, we have

$$
\inf _{\left\|\boldsymbol{\beta}_{\mathcal{S}}^{*}\right\|=L q_{n}^{1 / 2} k_{n}^{1 / 2}} \sum_{i=1}^{n}\left[\rho_{\tau}\left(\epsilon_{i}-\mathbf{Z}_{i, \mathcal{S}}^{\mathrm{T}} \boldsymbol{\beta}_{\mathcal{S}}^{*}-R_{i, \mathcal{S}}\right)-\rho_{\tau}\left(\epsilon_{i}-R_{i, \mathcal{S}}\right)\right] \geq q_{n} k_{n} / 2 .
$$

Thus, we have

$$
\mathrm{P}\left\{\inf _{\left\|\boldsymbol{\beta}_{\mathcal{S}}^{*}\right\|=L q_{n}^{1 / 2} k_{n}^{1 / 2}} \sum_{i=1}^{n} \rho_{\tau}\left(\epsilon_{i}-\mathbf{Z}_{i, \mathcal{S}}^{\mathrm{T}} \boldsymbol{\beta}_{\mathcal{S}}^{*}-R_{i, \mathcal{S}}\right)>\sum_{i=1}^{n} \rho_{\tau}\left(\epsilon_{i}-R_{i, \mathcal{S}}\right)\right\} \rightarrow 1,
$$

for $L>0$. By the convexity of quantile loss function, $\left\|\hat{\boldsymbol{\beta}}_{\mathcal{S}}^{*}-\boldsymbol{\beta}_{0, \mathcal{S}}^{*}\right\|=$ $O_{p}\left(q_{n}^{1 / 2} k_{n}^{1 / 2}\right)$. This completes the proof.

\section{Proof of Theorem 3.2}

Define

$$
\mathcal{G}=\left\{\mathbf{g}=\left(\mathbf{g}_{1}^{\mathrm{T}}, \ldots, \mathbf{g}_{p_{n}}^{\mathrm{T}}\right)^{\mathrm{T}}, \mathbf{g}_{l}=n \lambda_{n} \hat{\boldsymbol{\beta}}_{l} /\left\|\hat{\boldsymbol{\beta}}_{l}\right\|, l=1, \ldots, q_{n}\right.
$$




$$
\left.\mathbf{g}_{l}=\boldsymbol{\nu}_{l}(\hat{\boldsymbol{\beta}})+n \lambda_{n} \mathbf{b}_{l}, l=q_{n}+1, \ldots, p_{n}\right\}
$$

where $\left\|\mathbf{b}_{l}\right\| \leq 1$ for $l=q_{n}+1, \ldots, p_{n}$. From Lemma 3.4, we have

$$
\mathrm{P}\left(\mathcal{G} \subset \partial G_{n}(\hat{\boldsymbol{\beta}})\right) \rightarrow 1
$$

Consider any $\boldsymbol{\beta} \in \mathcal{B}\left(\hat{\boldsymbol{\beta}}, \lambda_{n} / 2\right)$. By Lemma 3.5, it is sufficient to show that there exists $\mathbf{g}^{*}=\left(\mathbf{g}_{1}^{* \mathrm{~T}}, \ldots, \mathbf{g}_{p_{n}}^{* \mathrm{~T}}\right)^{\mathrm{T}} \in \mathcal{G}$ and $\mathbf{h}^{*}=\left(\mathbf{h}_{1}^{* \mathrm{~T}}, \ldots, \mathbf{h}_{p_{n}}^{* \mathrm{~T}}\right)^{\mathrm{T}} \in \partial H_{n}(\boldsymbol{\beta})$ such that

$$
\mathrm{P}\left(\mathbf{g}_{l}^{*}=\mathbf{h}_{l}^{*}, l=1, \ldots, p_{n}\right) \rightarrow 1 .
$$

From Lemma 3.4, we have

$$
\left\|\boldsymbol{\beta}_{l}\right\| \geq\left\|\hat{\boldsymbol{\beta}}_{l}\right\|-\left\|\boldsymbol{\beta}_{l}-\hat{\boldsymbol{\beta}}_{l}\right\| \geq(a+1 / 2) \lambda_{n}-\lambda_{n} / 2=a \lambda_{n}
$$

for $l=1, \ldots, q_{n}$. For any $\boldsymbol{\beta} \in \mathcal{B}\left(\hat{\boldsymbol{\beta}}, \lambda_{n} / 2\right)$, we can find a $\boldsymbol{\beta}$ such that $\boldsymbol{\beta}_{l} /\left\|\boldsymbol{\beta}_{l}\right\|=\hat{\boldsymbol{\beta}}_{l} /\left\|\hat{\boldsymbol{\beta}}_{l}\right\|$ for $l=1, \ldots, q_{n}$. Let $\mathbf{h}_{l}^{*}=n \lambda_{n} \boldsymbol{\beta}_{l} /\left\|\boldsymbol{\beta}_{l}\right\|$ when $l=$ $1, \ldots, q_{n}$ and thus 3.14 holds in this case.

For $l=q_{n}+1, \ldots, p_{n}$, we have $\hat{\boldsymbol{\beta}}_{l}=\mathbf{0}$. Then,

$$
\left\|\boldsymbol{\beta}_{l}\right\| \leq\left\|\hat{\boldsymbol{\beta}}_{l}\right\|+\left\|\boldsymbol{\beta}_{l}-\hat{\boldsymbol{\beta}}_{l}\right\| \leq \lambda_{n} / 2<\lambda_{n} .
$$

Therefore, $\partial H_{n}(\boldsymbol{\beta}) / \partial \boldsymbol{\beta}_{l}=\mathbf{0}$. From Lemma 3.4. we have $\left\|\boldsymbol{\nu}_{l}(\hat{\boldsymbol{\beta}})\right\|<n \lambda_{n} / 2$, for $l=q_{n}+1, \ldots, p_{n}$. There exists $\mathbf{b}_{l}^{*}$ such that

$$
\mathrm{P}\left(\boldsymbol{\nu}_{l}(\hat{\boldsymbol{\beta}})+n \lambda_{n} \mathbf{b}_{l}^{*}=\mathbf{0}\right) \rightarrow 1 .
$$

Let $\mathbf{g}_{l}^{*}=\boldsymbol{\nu}_{l}(\hat{\boldsymbol{\beta}})+n \lambda_{n} \mathbf{b}_{l}^{*}$ and $\mathbf{h}_{l}^{*}=\mathbf{0}$. Then, we have

$$
\mathrm{P}\left(\mathbf{g}_{l}^{*}=\mathbf{h}_{l}^{*}\right) \rightarrow 1 .
$$


Chapter 4

\section{Discussion and Future Research}

In this thesis, two flexible semi-parametric quantile regression models have been studied. More specifically, we proposed two fused adaptive group LASSO penalties to induce interquantile shrinkage in additive regression models in Chapter 2. We considered variable selection for quantile additive coefficient models with high-dimensional covariates in Chapter 3, and established the oracle property of the estimator. Theoretical results and numerical studies demonstrated that the proposed approaches yielded better performance than some existing methods. We also applied the proposed methods to real data. In Chapter 2, we studied an environmental data and predicted the effects on the concentration of $\mathrm{NO}_{2}$ particles at various quantile levels. In Chapter 3, we investigated gene-environment interaction between sheep traits and SNPs, which contributed to the genome wide association study.

Regarding the simulation studies in Chapter 2, we considered two examples, where one example contains a non-parametric function $f_{3}$ varies among all the quantile levels that we are interested in and the other one example contains a non-parametric function $f_{2}$ varies when quantile level 
$\tau>0.49$. We notice that the two proposed methods have favourable performances with different settings. We can even construct a complicated example with a non-parametric function $f$, where $f$ varies among several quantile regions, such as $f$ varying when $0<\tau<\tau_{1}, \tau_{2}<\tau<1$ and unvarying when $\tau_{1}<\tau<\tau_{2}$. In this situation, it is expected that the FAGL1 will perform better than the FAGL2 in selection accuracy as Example 2.2 illustrated in Chapter 2 .

It is worth noting that we considered the interquantile shrinkage in Chapter 2 regarding a set of discrete quantile levels $\left\{\tau_{1}, \ldots, \tau_{K}\right\}$. Lian et al. (2015) proposed smoothed estimation of the conditional quantile process which estimated the parameters $\beta_{j}$ in linear models as continuous functions $\beta_{j}(\tau)$ towards quantile level $\tau$. Extensions that can be considered for future research includes smoothing estimation for the non-parametric components in additive quantile regression models 1.7 or the coefficient functions in additive coefficient models (1.10). For example, in additive quantile regression models (1.7), we may use tensor splines to approximate the multivariate functions $f_{j}\left(X_{j}, \tau\right), j=1, \ldots, p$. Technically, the estimation procedure would be complicated and hard to get asymptotic properties. We will consider this problem in the future. In addition, we can extend the current study in Chapter 2 and consider variable selection in additive quantile regression models by adding another penalty similar to that in Chapter 3.

For the oracle property, the theory in Chapter 3 is obviously incomplete. Following the general strategy of Sherwood and Wang (2016), we only showed that the oracle estimator in Chapter 3 is a local minimizer of the penalized functional. It is not clear how to establish the asymptotic property of the global minimizer, or the property of the estimator produced 
by the computational algorithm. It would be a challenging problem to be considered for future work. We note that the simulation results for the local minimizer in Chapter 3 perform well.

Another important problem that we did not discuss in this thesis is the problem of how to guarantee non-crossing quantile curves. Some constraints possibly need to be put on the spline coefficients (Bondell et al., 2010), or some post-processing techniques should be used (Dette and Volgushev, 2008; Chernozhukov et al., 2009). But extending these approaches to highdimensional semi-parametric models seems to be very challenging.

The methodologies we proposed in this thesis can be potentially extended to other semi-parametric models. For example, we can consider interquantile shrinkage and variable selection in single-index models, varying coefficient models and partially linear additive models with high dimensionality. 



\section{References}

Abrevaya, J. "The effects of demographics and maternal behavior on the distribution of birth outcomes." Empirical Economics, 26:247-257 (2001).

Belloni, A. and Chernozhukov, V. "l1-penalized quantile regression in high-dimensional sparse models." The Annals of Statistics, 39(1):82-130 (2011).

Bondell, H. D., Reich, B. J., and Wang, H. "Noncrossing quantile regression curve estimation." Biometrika, 97(4):825-838 (2010).

Breiman, L. and Friedman, J. "Estimating optimal transformations for multiple regression and correlation." Journal of the American Statistical Association, 80:580-598 (1985).

Buchinsky, M. "Change in the US wage structure 1963-1987: application of quantile regression." Econometrica: Journal of the Econometric Society, 62:405-458 (1994).

Buja, A., Hastie, T., and Tibshirani, R. "Linear smoothers and additive models." The Annals of Statistics, 17(2):453-510 (1989). 
Chernozhukov, V., Fernandez-Val, I., and Galichon, A. "Improving point and interval estimators of monotone functions by rearrangement." Biometrika, 96(3):559-575 (2009).

De Gooijer, J. G. and Zerom, D. "On additive conditional quantiles with high-dimensional covariates." Journal of the American Statistical Association, 98(461):135-146 (2003).

Dette, H. and Volgushev, S. "Non-crossing non-parametric estimates of quantile curves." Journal of the Royal Statistical Society: Series B (Statistical Methodology), 70(3):609-627 (2008).

Fan, J., Fan, Y., and Barut, E. "Adaptive robust variable selection." The Annals of Statistics, 42:324-351 (2014a).

Fan, J. and Li, R. "Variable selection via nonconcave penalized likelihood and its oracle properties." Journal of the American Statistical Association, 96:1348-1360 (2001).

Fan, J., Ma, Y., and Dai, W. "Nonparametric independence screening in sparse ultra-high dimensional varying coefficient models." Journal of the American Statistical Association, 109:1270-1284 (2014b).

Friedman, J. and Stuetzle, W. "Projection pursuit regression." Journal of the American Statistical Association, 76:817-823 (1981).

Hastie, T. and Tibshirani, R. "Varying-coefficient models." Journal of the Royal Statistical Society Series B-Statistical Methodolog, 55:757-796 (1993). 
He, X. and Shi, P. "Convergence rate of B-spline estimators of nonparametric conditional quantile functions." Journal of Nonparametric Statistics, 3:299-308 (1994).

Horowitz, J. and Lee, S. "Nonparametric estimation of an additive quantile regression model." Journal of the American Statistical Association, 100:1238-1249 (2005).

Huang, J., Horowitz, J., and Wei, F. "Variable selection in nonparametric additive models." The Annals of Statistics, 38:2282-2313 (2010).

Hunter, D. and Lange, K. "Quantile regression via an MM algorithm." Journal of Computational and Graphical Statistic, 9:60-77 (2000).

Jiang, L., Bondell, H., and Wang, H. "Interquantile shrinkage and variable selection in quantile regression." Computational Statistics and Data Analysis, 69:208-219 (2014).

Jiang, L., Wang, H., and Bondell, H. "Interquantile shrinkage in regression models." Journal of Computational and Graphical Statistics, 22:970-986 (2013).

Kim, M. "Quantile regression with varying coefficients." Annals of Statistics, 35(1):92-108 (2007).

Koenker, R. Quantile regression. Econometric Society monographs. Cambridge ; New York: Cambridge University Press (2005).

Koenker, R. and Bassett, G. "Regression quantiles." Econometrica, 46:3350 (1978). 
Koenker, R. and Mizera, I. "Penalized triograms: total variation regularization for bivariate smoothing." Journal of the Royal Statistical Society: Series B, 66:145-163 (2004).

Koenker, R., Ng, P., and Portnoy, S. "Quantile smoothing splines." Biometrika, 81:673-680 (1994).

Lian, H. "Semiparametric estimation of additive quantile regression models by two-fold penalty." Journal of Business 86 Economic Statistics, 30(3):337-350 (2012).

Lian, H., Chen, X., and Yang, J. "Identification of partially linear structure in additive models with an application to gene expression." Biometrics, 68(2):437-445 (2012).

Lian, H., Meng, J., and Fan, Z. "Simultaneous estimation of linear conditional quantiles with penalized splines." Journal of Multivariate Analysis, 141:1-21 (2015).

Linton, O. and Nielsen, J. P. "A kernel method of estimating structured nonparametric regression based on marginal integration." Biometrika, 82(1):93-100 (1995).

Liu, R. and Yang, L. "Spline-backfitted kernel smoothing of additive coefficient model." Econometric Theory, 26:29-59 (2010).

Noh, H., Chung, K., and Van Keilegom, I. "Variable selection of varying coefficient models in quantile regression." Electronic Journal of Statistics, 6:1220-1238 (2012).

Picard, R. and Cook, D. "Cross-Validation of regression models." Journal of the American Statistical Association, 79:575-583 (1984). 
Schumaker, L. Spline functions: basic theory. Cambridge, UK: Cambridge University Press (2007).

Schwarz, G. "Estimating the dimension of a model." The Annals of Statistics, 6:461-464 (1978).

Sherwood, B. and Wang, L. "Partially linear additive quantile regression in unltra-high dimension." The Annals of Statistics, 44:288-317 (2016).

Stone, C. "Additive regression and other nonparametric models." The Annals of Statistics, 13:689-705 (1985).

Tao, P. and An, L. "Convex analusis approach to D.C. programming: theory, algorithms and applications." Acta Mathematica Vietnamica, 22:289-355 (1997).

Tibshirani, R. "Regression shrinkage and selection via the lasso." Journal of the Royal Statistical Society: Series B, 58:267-288 (1996).

Tibshirani, R., Saunders, M., Rosset, S., and Zhu, J. "Sparsity and smoothness via the fussed lasso." Journal of the Royal Statistical Society: Series $B, 67: 91-108$ (2005).

van der Geer, S. A. Empirical Processes in M-Estimation. Cambridge: Cambridge University Press (2000).

Waldmann, E., Kneib, T., Yue, Y. R., Lang, S., and Flexeder, C. "Bayesian semiparametric additive quantile regression." Statistical Modelling, 13(3):223-252 (2013).

Wang, H., Zhu, Z., and Zhou, J. "Quantile regression in partially linear varying coefficient models." The Annals of Statistics, 37:3841-3866 (2009). 
Wang, L., Liu, X., Liang, H., and Carroll, R. "Estimation and variable selection for generalized additive partial linear models." The Annals of Statistics, 39(4):1827-1851 (2011).

Wang, L., Wu, Y., and Li, R. "Quantile regression for analyzing heterogeneity in ultra-high dimension." Journal of the American Statistical Association, 107:214-222 (2012).

Wang, L. and Yang, L. "Spline-backfitted kernel smoothing of nonlinear additive autoregression model." The Annals of Statistics, 35:2474-2503 (2007).

Xia, Y., Tong, H., Li, W., and Zhu, L. "An adaptive estimation of dimension reduction space." Journal of the Royal Statistical Society: Series B, 64:363-410 (2002).

Xue, L. "Consistent variable selection in additive models." Statistica Sinica, 19:1281-1296 (2009).

Xue, L. and Yang, L. "Additive coefficient modeling via polynomial spline." Statistica Sinica, 16:1423-1446 (2006a).

—. "Estimation of semi-parametric additive coefficient model." Journal of Statistical Planning and Inference, 136:2506-2534 (2006b).

Yang, Y. and Zou, H. "A fast unified algorithm for solving group-lasso penalized learning problems." Statistics and Computing, 25:1129-1141 (2015).

Yuan, M. and Lin, Y. "Model selection and estimation in regression with grouped variables." Journal of the Royal Statistical Society: Series B, 68:49-67 (2006). 
Zhang, C. "Nearly unbiased variable selection under minimax concave penalty." The Annals of Statistics, 38(2):894-942 (2010).

Zhang, H., Cheng, G., and Liu, Y. "Linear or nonlinear? Automatic structure discovery for partially linear models." Journal of the American Statistical Association, 106(495):1099-1112 (2011).

Zhang, L., Liu, J., Zhao, F., Ren, H., and Xu, L. "Genome-Wide association studies for growth and meat production traits in sheep." PLoS ONE, 8:e66569 (2013).

Zhao, P. and Yu, B. "On model selection consistency of Lasso." Journal of Machine Learning Research, 7:2541-2563 (2006).

Zhao, W. and Lian, H. "Quantile index coefficient model with variable selection." Journal of Multivariate Analysis, 154:4058 (2017).

Zou, H. "The adaptive lasso and its oracle properties." Journal of the American Statistical Association, 101:1418-1429 (2006). 